\title{
Principes directeurs de l'OCDE pour la prévention, la préparation et l'intervention en matière d'accidents chimiques
}

Document d'orientation à l'intention de l'industrie (incluant direction et travailleurs), des pouvoirs publics, des collectivités et d'autres parties prenantes

Deuxième édition 


\section{ORGANISATION DE COOPÉRATION ET DE DÉVELOPPEMENT ÉCONOMIQUES}

En vertu de l'article 1 de la Convention signée le 14 décembre 1960, à Paris, et entrée en vigueur le 30 septembre 1961, l'Organisation de coopération et de développement économiques (OCDE) a pour objectif de promouvoir des politiques visant :

- à réaliser la plus forte expansion de l'économie et de l'emploi et une progression du niveau de vie dans les pays membres, tout en maintenant la stabilité financière, et à contribuer ainsi au développement de l'économie mondiale;

- à contribuer à une saine expansion économique dans les pays membres, ainsi que les pays non membres, en voie de développement économique; et,

- à contribuer à l'expansion du commerce mondial sur une base multilatérale et non discriminatoire conformément aux obligations internationales.

Les pays membres à l'origine de l'OCDE sont : l'Allemagne, l'Autriche, la Belgique, le Canada, le Danemark, l'Espagne, les États-Unis, la France, la Grèce, l'Irlande, l'Islande, l'Italie, le Luxembourg, la Norvège, les Pays-Bas, le Portugal, le Royaume-Uni, la Suède, la Suisse et la Turquie. Les pays suivants sont ultérieurement devenus membres par adhésion aux dates indiquées ci-après : le Japon (28 avril 1964), la Finlande (28 janvier 1969), l'Australie (7 juin 1971), la Nouvelle-Zélande (29 mai 1973), le Mexique (18 mai 1994), la République tchèque (21 décembre 1995), la Hongrie (7 mai 1996), la Pologne (22 novembre 1996), la Corée (2 décembre 1996) et la Slovaquie (14 décembre 2000). La Commission des Communautés européennes participe aux travaux de l'OCDE (article 13 de la Convention de l'OCDE).

Les permissions de reproduction partielle à usage non commercial ou destinée à une formation doivent être adressées au Centre français d'exploitation du droit de copie (CFC), 20, rue des Grands-Augustins, 75006 Paris, France, tél. : (33-1) 44 07 47 70, fax : (33-1) 463467 19, pour tous les pays à l'exception des États-Unis. Aux États-Unis, l'autorisation doit être obtenue du Copyright Clearance Center, Service Client, (508) 750-8400, 222 Rosewood Drive, Danvers, MA 01923 USA, ou CCC Online : www.copyright.com. Toute autre demande d'autorisation de reproduction ou de traduction totale ou partielle de cette publication doit être adressée aux Éditions de l'OCDE, 2, rue André-Pascal, 75775 Paris Cedex 16, France. 


\section{PRÉFACE}

L'industrie chimique produit de nombreuses substances utiles, sans lesquelles la société moderne ne pourrait fonctionner comme elle le fait actuellement. Mais, la production, l'entreposage, le transport, l'utilisation et l'élimination des produits chimiques peuvent aussi entraîner des risques et conduire à des accidents majeurs. Une ville entière peut être menacée par une explosion dans une usine chimique, toute une côte par le naufrage d'un pétrolier, et toute une région par les fuites dans une installation chimique. Bhopal (1984) a été la scène de l'accident qui a causé le plus grand nombre de victimes humaines; l'incendie de l'entrepôt de Bâle (1986) a entraîné une pollution à grande échelle du Rhin; le déversement de Baia Mare (2000) a gravement menacé le Danube. Plus récemment, les habitants et les villes d'Enschede (2000) et de Toulouse (2001) ont été gravement affectés par des explosions chimiques.

À la Conférence de l'OCDE sur les accidents liés à des substances dangereuses (1988), les ministres ont mis en œuvre un programme ambitieux pour l'OCDE dans ce domaine. Quatre actes du Conseil élaborés dans le cadre de ce programme ont facilité le choix de politiques pour faire face aux accidents majeurs dans les pays membres. De plus, les Principes directeurs de l'OCDE pour la prévention, la préparation et l'intervention en matière d'accidents chimiques ont été publiés en 1992. Des experts des gouvernements, de l'industrie, des organisations syndicales, des groupes écologiques et d'autres organisations internationales ont collaboré étroitement à l'élaboration du présent document. L'application des Principes directeurs est l'objet d'une Recommandation du Conseil de l'OCDE; ils ont été traduits dans de nombreuses langues et sont aussi largement utilisés dans des pays non-membres.

Au cours des dix dernières années, les gouvernements et l'industrie ont axé leurs efforts sur la mise en oeuvre de ces Principes directeurs. Parallèlement, le Groupe de travail sur les accidents chimiques de l'OCDE a amélioré et mis à jour les principes en se fondant sur de nouvelles expériences et en étendant les principes de façon à couvrir de nouveaux sujets. Cela a été réalisé grâce à de nombreux ateliers organisés par l'OCDE, avec la participation des parties prenantes. Ces travaux ont abouti à la seconde édition des Principes directeurs qui contiennent maintenant de nouveaux éléments dans des domaines additionnels, comme les suivants: création d'une infrastructure sanitaire pour faire face aux accidents chimiques; application des principes par les petites et moyennes entreprises; sécurité chimique aux interfaces des transports, comme les zones portuaires; sécurité des transports par pipelines; gestion intégrée de la santé, de l'environnement, de la sécurité et du contrôle de la qualité; orientation-conseils en matière d'audits et d'inspections; et, application au sabotage et au terrorisme.

Cette seconde édition des Principes directeurs aidera, encore plus que la première version, les pouvoirs publics, l'industrie et les collectivités à l'échelle mondiale à prévenir les accidents chimiques et à améliorer les étapes de préparation et $\mathrm{d}$ 'intervention en cas d'accident. Je pense que le présent document est un excellent exemple de la façon dont l'OCDE peut réunir des experts de nombreux secteurs de la société pour mettre sur pied un outil très pratique, qui sera d'une grande utilité aussi bien pour les pays membres que non-membres. Le document deviendra certainement une étape importante sur le chemin menant à la sécurité en matière chimique dans le monde. 


\section{REMERCIEMENTS}

Cette deuxième édition des Principes directeurs de l'OCDE pour la prévention, la préparation et l'intervention en matière d'accidents chimiques a été préparée par un groupe de rédaction oeuvrant sous les auspices du Groupe de travail sur les accidents chimiques, qui gère le Programme de l'OCDE sur les accidents chimiques. L'élaboration des Principes directeurs a été entreprise en collaboration avec d'autres organisations internationales oeuvrant dans le domaine de la prévention, de la préparation et de l'intervention en matière d'accidents chimiques, et notamment les suivantes: OIT, OMI, CEE-ONU, PNUE, PNUE-BCAH (Groupe commun PNUE/BCAH de l'environnement) et OMS.

L'élaboration des Principes directeurs de l'OCDE a bénéficié des connaissances étendues et de la vaste expérience du Groupe de rédaction, qui comprenait des représentants des pays membres de l'OCDE et de pays présents comme observateurs, de la Commission européenne, de l'industrie, des travailleurs, d'organisations non gouvernementales et d'autres organisations internationales. Le groupe s'est réuni six fois entre 1999 et 2002, sur invitation gracieuse du Canada, de l'Italie, des ÉtatsUnis et de la Commission européenne. Les membres du Groupe de rédaction étaient les suivants : Wayne Bissett, Eric Clément, Jean-Paul Lacoursière et Robert Reiss (Canada); Jukka Metso (Finlande); David Hourtolou et Olivier Salvi (France); Erika Moch et Jorg Steinbach (Allemagne); Roberta Gagliardi, Gian Carlo Ludovisi et Raffaele Scialdoni (Italie); Soon-Joong Kang, Jae-Hyyn Kim et Hyuck Myun Kwon (Corée); Gunnar Hem (Norvège); Mieczyslaw Borysiewicz et Barbara Kucnerowicz Polak (Pologne); Josef Skultety (Slovaquie); Juan Pablo Perez Sanchez (Espagne); Äke Persson (Suède); David Bosworth (R.-U.); Kim Jennings, Kathy Jones et Jim Makris (États-Unis); Juergen Wettig (Commission européenne); Sigal Blumenfeld (Israël); Apostolos Paralikas (BEE); Fritz Balkau et Ruth Do Coutto (PNUE); Patricia Charlebois et Vladimir Sakharov (PNUE-BCAH); Kersten Gutschmidt (OMS- PISSC); Simon Cassidy (BIAC); Reg Green (TUAC).

Francine Schulberg (consultante de l'OCDE) a rédigé et édité le document. Peter Kearns, Béatrice Grenier et Marie-Chantal Huet (Secrétariat de l'OCDE) se sont occupés du suivi pour l'ensemble du processus, sous la supervision de Robert Visser. Le document a également été édité par Beatrix de Koster.

À la fin du processus d'élaboration des Principes directeurs, une séance élargie d'échanges de commentaires sur l'ébauche finale et une réunion pour un examen spécial ont été organisées. Des experts du monde entier ont fait part de leurs commentaires au sujet du document. Parmi les participants de cet examen par les pairs, on peut citer les suivants : Nestor H. Sposito (Argentine); Jose A. Coelho et Cesar A. Leal (Brésil); Wayne Bissett, Jean-Paul Lacoursière et Robert Reiss (Canada); Olivier Salvi (France); Mark Hailwood (Allemagne); Apostolos Paralikas et Elias Sampatakakis (Grèce); Elena Floridi, Roberta Gagliardi, Gian Carlo Ludovisi et Raffaele delle Piane (Italie); Hyuck Myun Kwon (Corée); Luis Hector Barojas Weber (Mexique); Gerard Lommers (PaysBas); Gunnar Hem (Norvège); Manuel Bouza Serrano (Portugal); Henrieta Lefflerova (Slovaquie); Garcia Ara, Gonzalo del Castillo, Marisol Lorente, Francisco Perez, Agata ML Puente Rubio et Olga Sanahuja (Espagne); Äke Persson (Suède); Bernard Gay (Suisse); David Bosworth, Simon Cassidy, Reg Green, Elisabeth Schoffield (R.-U.); Kim Jennings, Kathy Jones, Dorothy McManus, Jim Makris et vingt autres examinateurs (États-Unis); Juergen Wettig (EC); Fritz Balkau, Ruth Do Coutto, James Kamara, David Thwaites et Jiang Yangpin (PNUE).

La version interactive des Principes directeurs pour Internet a été élaborée avec l'aide de l'EPA des États-Unis (sous la conduite de Kim Jennings) et le concours de Francine Schulberg. 
Une brochure pour la promotion des Principes directeurs et du Document d'orientation sur les indicateurs de performance en matière de sécurité connexe a été élaborée avec l'aide de Dana Robinson, Kim Jennings, Kathy Jones et Francine Schulberg.

L'élaboration des Principes directeurs a été rendue possible grâce à des contributions extrabudgétaires de l'Autriche, du Canada, de la Finlande, de l'Allemagne, des Pays-Bas, de la Norvège, de la Suisse et des États-Unis.

La présente publication est dédiée à la mémoire de Jim Makris et à son esprit d'initiative, son enthousiasme et son dévouement pour la coopération internationale dans le domaine de la prévention, de la préparation et de l'intervention en matière d'accidents chimiques, pour le Programme de l'OCDE sur les accidents chimiques, et enfin pour l'élaboration de ces Principes directeurs. 


\section{À PROPOS DE L'OCDE ET DE L'IOMC}

L'Organisation de coopération et de développement économiques (OCDE) est une organisation intergouvernementale au sein de laquelle des représentants de 30 pays industrialisés (d'Europe, d'Amérique du Nord et du Pacifique) ainsi que de la Commission européenne se réunissent afin de coordonner et d'harmoniser leurs politiques, d'examiner des questions d'intérêt commun et de coopérer à la résolution de problèmes internationaux. La majeure partie des travaux de l'OCDE sont menés à bien par plus de 200 comités spécialisés et groupes subsidiaires composés de délégués des pays membres. Des observateurs de différents pays possédant un statut spécial auprès de l'OCDE, et d'organisations internationales intéressées assistent à un grand nombre des réunions de l'OCDE. Le Secrétariat de l'OCDE, qui a son siège à Paris (France), assiste les comités et les groupes subsidiaires et se compose de directions et de divisions.

Le travail de l'OCDE relatif à la prévention, à la préparation et à l'intervention en matière d'accidents chimiques est effectué par le Groupe de travail sur les accidents chimiques (GTAC), avec le soutien administratif de la Division environnement, santé et sécurité (ESS), relevant de la Direction de l'environnement ${ }^{1}$. Les objectifs du programme sur les accidents chimiques comprennent l'élaboration de notes d'orientation liées à la prévention, à la préparation et à l'intervention en matière d'accidents chimiques, à l'échange d'information et d'expérience, et à l'analyse de questions spécifiques présentant un intérêt mutuel pour les pays membres de l'OCDE. Dans ce contexte, plus de quinze ateliers et sessions spéciales ont été organisés depuis 1989.

Dans le cadre de ses activités concernant les accidents chimiques, l'OCDE a publié plusieurs Décisions et Recommandations du Conseil (les premières ayant force de loi dans les pays membres), ainsi que de nombreux documents d'orientation et rapports techniques. Parmi les publications additionnelles, il y a les suivantes : Document d'orientation sur les indicateurs de performance en matière de sécurité (publié en 2003); Directives relatives à la sécurité des substances chimiques dans les zones portuaires (une initiative conjointe avec l'OMI); Orientations concernant les aspects des accidents chimiques touchant à la santé; Répertoire international des centres d'intervention d'urgence, document conjoint de l'OCDE/PNUE/BCAH; et des rapports sur les divers ateliers.

La présente publication a été préparée dans le cadre du Programme interorganisations pour la gestion rationnelle des produits chimiques (IOMC). L'IOMC a été créé en 1995 par le PNUE, l'OIT, l'OAA, l'OMS, l'ONUDI et l'OCDE (Organisations participantes), suite aux recommandations de la Conférence des Nations Unies sur l'environnement et le développement tenue en 1992 pour renforcer la coopération et accroître la coordination internationale dans le domaine de la sécurité chimique. En 1997, l'UNITAR a adhéré à l'IOMC et est devenu la septième Organisation participante. L'objectif de l'IOMC est de promouvoir la coordination des politiques et des activités poursuivies, conjointement ou séparément, par les Organisations participantes, visant à atteindre une gérance des produits chimiques pour la santé et l'environnement.

Obtention des publications de l'OCDE : le document intitulé Principes directeurs de l'OCDE pour la prévention, la préparation et l'intervention en matière d'accidents chimiques est disponible gratuitement sous forme électronique. Pour en obtenir le texte complet ou celui d'autres publications du Programme sur l'hygiène et la sécurité de l'environnement, veuillez consulter le site Internet de l'OCDE (www.oecd.org/ehs/) ou communiquer avec: la Direction de l'environnement de l'OCDE (Division environnement, santé et sécurité), 2, rue André-Pascal, 75775 Paris Cedex 16, France. Fax: (33) 145241675. Courriel : ehscont@oecd.org 


\section{TABLE DES MATIÈRES}

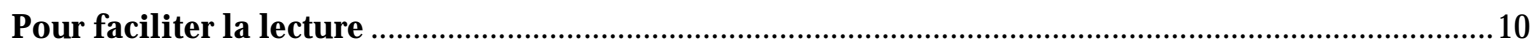

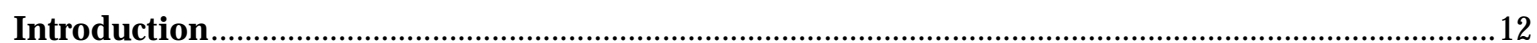

Au sujet de cette publication ..........................................................................................12

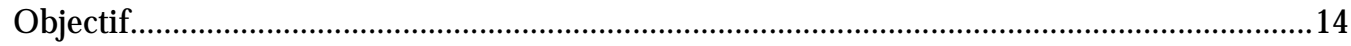



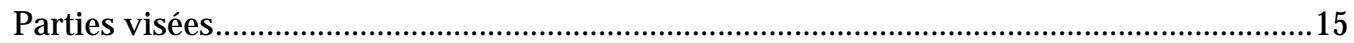

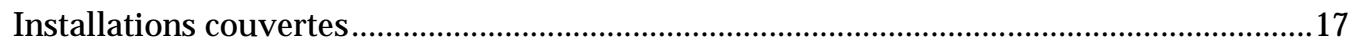

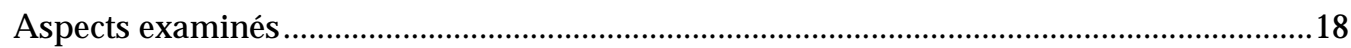

Application à l'échelle mondiale ........................................................................................19

Les « Règles d'or »



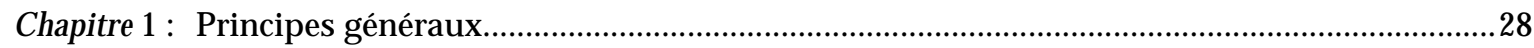

Chapitre 2: Industrie (incluant la direction et les travailleurs) …………………………..........................33

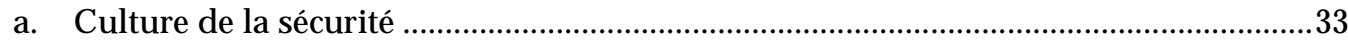

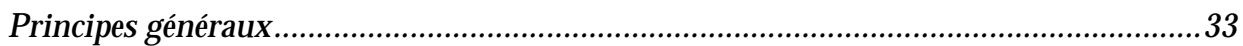



Systèmes de gestion de la sécurité ...................................................................................36

Rapports sur la sécurité

b. Identification des dangers et évaluation des risques .......................................................39

c. Implantation, conception et construction .....................................................................42

Implantation des installations .....................................................................................42

Conception, planification et aménagement......................................................................43

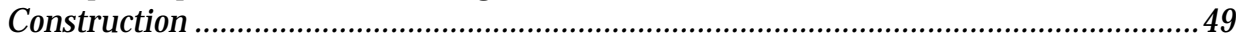



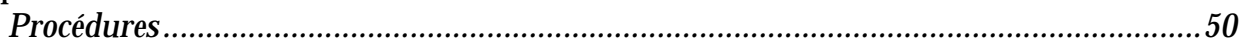

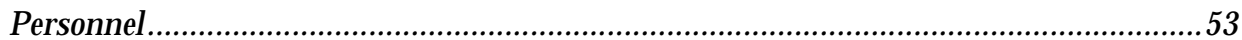

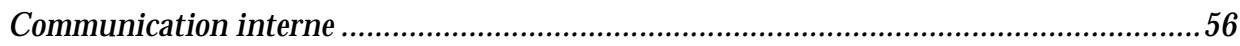

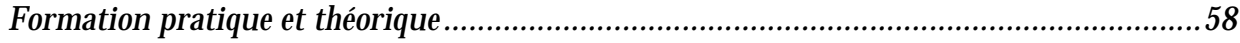



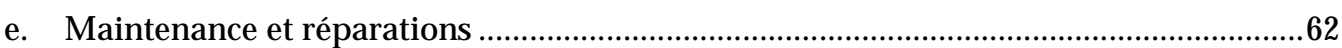

f. Modifications (techniques et organisationnelles) .............................................................63

g. Examen et évaluation de la performance en matière de gestion de la sécurité.............64

h. Mise hors service, fermeture et démolition .................................................................68



Gérance des produits et aide aux autres entreprises.......................................................68

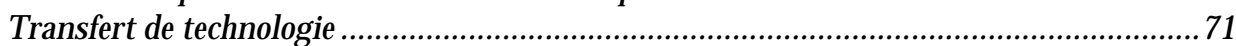

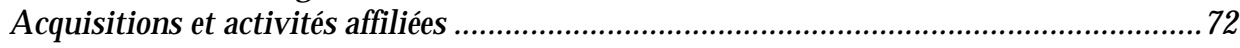

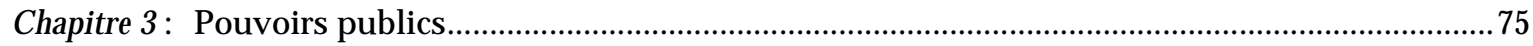

a. Stratégie en matière de sécurité et cadre de contrôle........................................................75

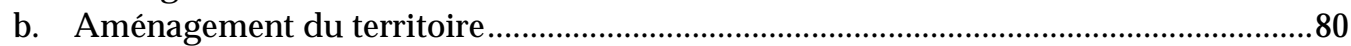

c. Examen et évaluation de la performance en matière de sécurité.....................................82

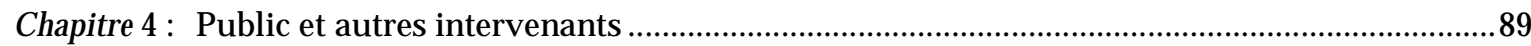

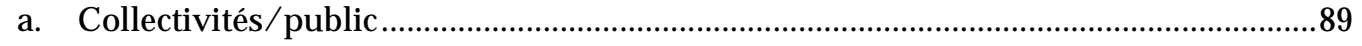




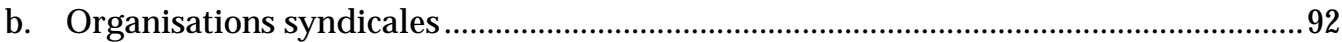

c. Établissements de recherche universitaires et autres ................................................92

d. Organisations internationales .............................................................................. 92

e. Organisations non gouvernementales (ONG) .......................................................92

Partie B : Préparation aux situations d'urgence et atténuation de leurs conséquences..................97

Chapitre 5 : Préparation et planification pour les situations d'urgence ...............................................98

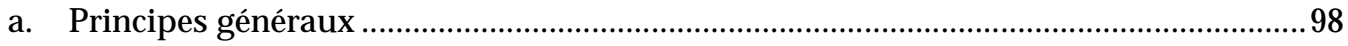

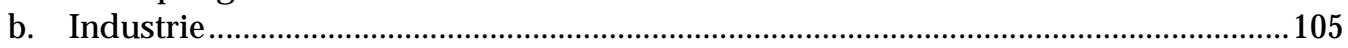

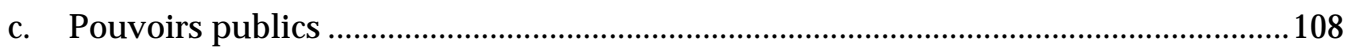

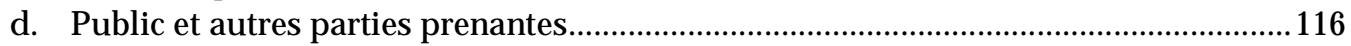

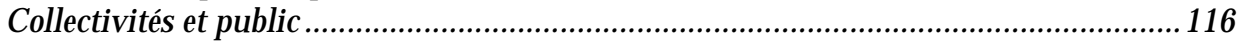

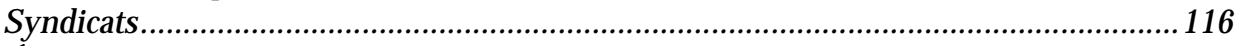

Établissements de recherche universitaires et autres .................................................. 117

Organisations non gouvernementales (ONG) ....................................................... 118

Chapitre 6 : Aménagement du territoire ......................................................................................... 119





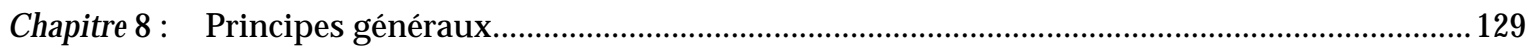

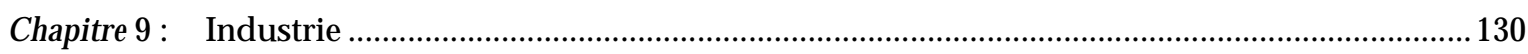

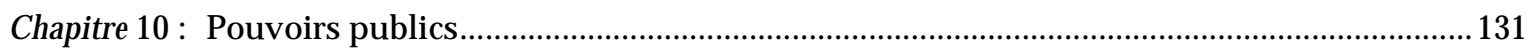

Chapitre 11 : Public et autres parties prenantes ............................................................................ 137

a. Public et collectivités ........................................................................................ 137

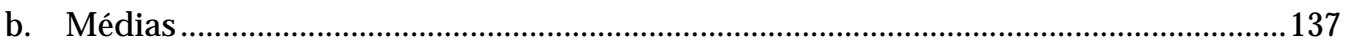

c. Organisations non gouvernementales (ONG) ..................................................... 137

Partie D : Suivi des incidents (Accidents et quasi-accidents) .................................................. 140

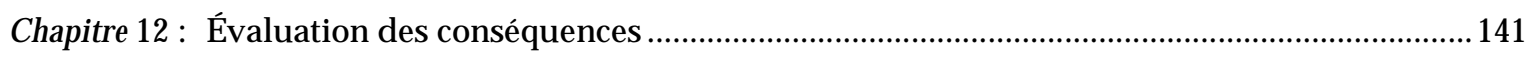

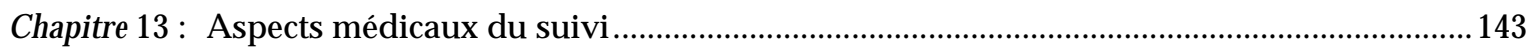

Chapitre 14 : Constitution de dossiers sur les incidents et déclarations ............................................ 144

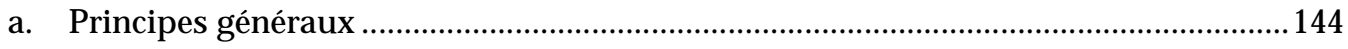

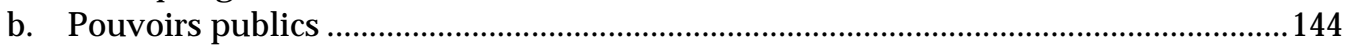



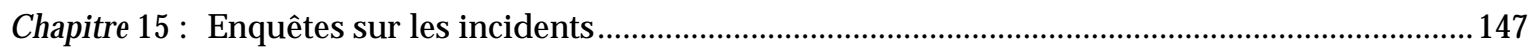



Éléments clés des enquêtes sur les causes fondamentales.........................................149

Partage des résultats de l'enquête ...................................................................... 151

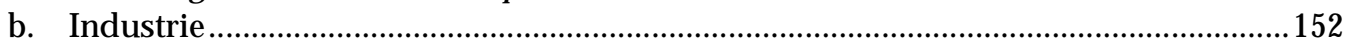




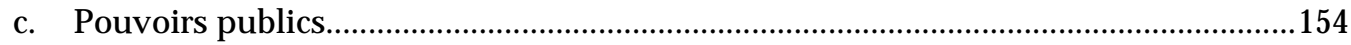

d. Autres parties prenantes .................................................................................... 155

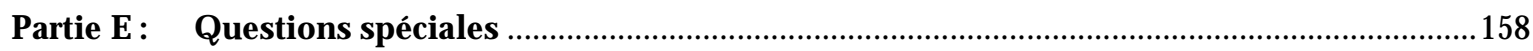

Chapitre 16: Questions transfrontalières/internationales ...............................................................159

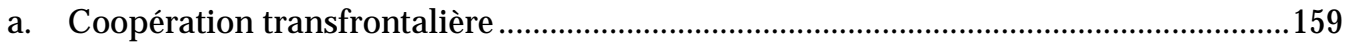

b. Aide technique et financière, bilatérale ou multilatérale ...........................................161

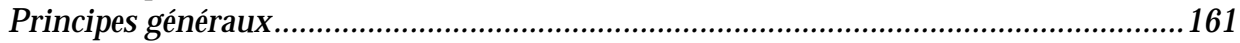

Rôle des organismes d'aide (nationaux et multinationaux) ...........................................163

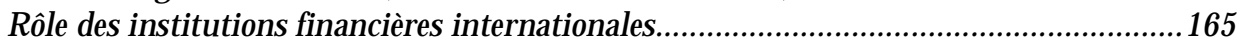

Rôle des organisations intergouvernementales ....................................................166

Rôle des pays bénéficiaires..............................................................................167

c. Transfert de technologie et investissement international..........................................168

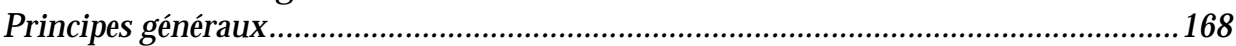

Transfert de technologie des pays développés vers les pays en développement ou à économie

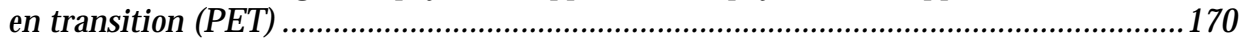

Investissement par des entreprises des pays développés dans des installations dangereuses établies dans des pays en développement ou à économie en transition (PET) ...................173

Rôle de l'industrie dans les pays bénéficiaires de la technologie ou de l'investissement ..175 Rôle des pouvoirs publics dans les pays bénéficiaires de la technologie ou de



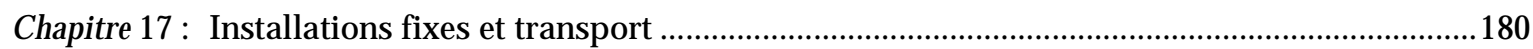

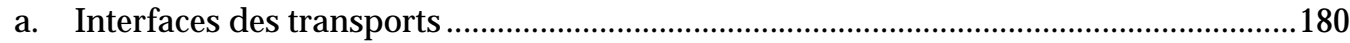

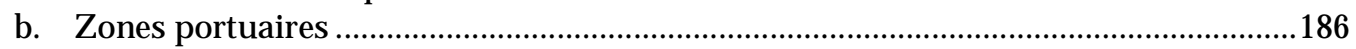








\section{POUR FACILITER LA LECTURE}

Voici la seconde édition des Principes directeurs de l'OCDE. Elle est disponible en copie papier et sur le site Internet de l'OCDE. Il y a un certain nombre de changements - aussi bien en format qu'en contenu - par rapport à la première édition. La présente édition est divisée en cinq parties principales, à la suite de l'introduction. Ces cinq parties portent sur les sujets suivants :

A. Prévention - Cette section inclut tous les aspects de la gestion, du fonctionnement et du contrôle d'une installation de substances dangereuses, à partir de sa conception jusqu'à sa mise hors service/démolition.

B. Préparation/atténuation - Cette partie est axée sur la planification de la préparation, la communication avec le public, ainsi que l'aménagement du territoire et l'implantation des installations.

C. Intervention - Concerne toutes les mesures prises une fois qu'un accident est arrivé ou s'il y a menace imminente d'un accident, ce qui comprend l'atténuation des effets nocifs sur la santé, l'environnement et les biens.

D. Suivi des incidents - Inclut les productions de rapports, les enquêtes et les activités de suivi médical.

E. Questions spéciales - Fournit de l'orientation additionnelle sur les questions transfrontalières ou internationales et sur les transports utilisant des installations fixes (c.-à-d. les installations fixes employées pour le transport des substances dangereuses, comme les pipelines, les zones portuaires, les gares de triage des chemins de fer et d'autres interfaces des transports).

NOTE : La présente orientation s'applique à toutes les installations de substances dangereuses, quelle qu'en soit la taille. Cependant, pour faciliter son application aux petites et moyennes entreprises (PME), un encadré texte à la fin du chapitre 1 met en évidence certains points qui peuvent intéresser tout particulièrement les PME et d'autres organismes qui travaillent avec les PME.

\section{Six annexes facilitent la lecture de la présente publication :}

Une Explication des termes utilisés (annexe I). Pour bien comprendre les Principes directeurs, il est important de consulter cette annexe. À noter, cependant, que les termes retenus ont été définis uniquement pour mieux comprendre et utiliser la présente publication; ces définitions ne doivent donc pas être considérées comme des définitions acceptées universellement, et il ne s'agit pas de termes harmonisés entre divers pays et organisations.

Une Liste d'acronymes (annexe II) donne le nom complet de la plupart des acronymes les plus couramment utilisés dans le contexte de la prévention, de la préparation et de l'intervention en matière d'accidents chimiques.

Un Index de mots-clés (annexe III) a été élaboré pour indiquer au lecteur les paragraphes qui traitent d'un sujet ou d'une partie en particulier. Les références croisées de cette annexe renvoient à des concepts apparentés (mais pas nécessairement identiques). En utilisant cet index, il faut tenir compte du fait que les différentes parties des Principes directeurs emploient parfois des termes légèrement différents, même lorsqu'on traite du même sujet ou d'un domaine très proche.

La Sélection de références (annexe IV) présente les principales publications internationales traitant de la prévention, de la préparation et de l'intervention en matière d'accidents chimiques, ainsi que l'information nécessaire pour obtenir les publications. Les références donnent aussi 
l'information (y compris les adresses Internet) sur les personnes-ressources d'un certain nombre d'organisations internationales oeuvrant dans les domaines couverts par les Principes directeurs.

L'Information documentaire (annexe $\mathrm{V}$ ) décrit brièvement les processus et les personnes qui ont travaillé à l'élaboration des Principes directeurs et présente une brève vue d'ensemble de l'OCDE.

Un Résumé des « Règles d'or » (annexe VI) reprend les principaux thèmes des Règles d'or (soit le texte mis en relief). Le texte complet des Règles d'or se trouve après l'introduction.

Note :

Comme l'OCDE prévoit examiner et réviser régulièrement les Principes directeurs, elle souhaiterait qu'on lui fasse part des commentaires sur le contenu et la présentation de cette publication. Si vous avez des commentaires ou des suggestions, veuillez communiquer avec le Secrétariat de l'OCDE à l'adresse suivante :

OCDE, Direction de l'environnement, Division de l'environnement, de la santé et de la sécurité

2, rue André-Pascal

75775 Paris Cedex 16

FRANCE

Fax : (33) 145241675

Courriel:ehscont@oecd.org 


\section{INTRODUCTION}

\section{Au sujet de cette publication}

Ces Principes directeurs ont été élaborés dans le cadre du Programme de l'OCDE sur les accidents chimiques et ont été produits dans le Programme interorganisations pour la gestion rationnelle des produits chimiques (IOMC). L'objectif est de déterminer une orientation générale pour la planification et le fonctionnement sûrs des installations où il y a présence de substances dangereuses, de façon à prévenir les accidents et, en admettant que des accidents mettant en jeu des substances dangereuses peuvent néanmoins se produire, d'atténuer les effets nocifs grâce à une préparation efficace en vue des situations d'urgence, à l'aménagement du territoire et à l'intervention en cas d'accident.

Les Principes directeurs s'adressent à toutes les parties prenantes (ou intervenants), définies pour les besoins de la présente publication comme étant tout individu, groupe ou organisation qui est concerné, intéressé ou potentiellement affecté par la prévention, la préparation et l'intervention en matière $d$ 'accidents chimiques. Le terme partie prenante englobe toute personne qui a un rôle, des responsabilités et des droits liés à la sécurité chimique ${ }^{2}$, notamment la direction des installations de substances dangereuses, les employés de ces installations, les pouvoirs publics à tous les niveaux, les membres des collectivités et du public, et d'autres parties intéressées ${ }^{3}$.

Les Principes directeurs sont conçus de façon à être complets et à répondre à divers éléments liés aux processus suivants :

" prévention des incidents (accidents et quasi-accidents ${ }^{4}$ ) qui mettent en cause des substances dangereuses ( $p$. ex. perte de l'enveloppe de confinement des substances dangereuses, explosions, incendies, etc.);

" préparation pour les situations d'accidents, et atténuation des effets néfastes des accidents, grâce à l'élaboration de plans d'urgence, à l'aménagement du territoire ${ }^{5}$ et à la communication avec le public;

" intervention en cas d'accident afin de réduire au minimum les conséquences nuisibles pour la santé, l'environnement et les biens;

" suivi après un accident, comprenant les activités initiales de nettoyage, la déclaration de l'accident et l'enquête.

Les Principes directeurs portent sur le risque d'événements imprévus mettant en cause des substances dangereuses à n'importe quelle installation, c.-à-d. à des installations fixes où des substances dangereuses sont produites, traitées, utilisées, manipulées, entreposées ou éliminées (elles sont appelées installations dangereuses dans la présente publication).

L'orientation donnée dans la présente publication vise également le transport de substances dangereuses relevant de la gestion des installations de substances dangereuses, ainsi que les installations fixes servant au transport de substances dangereuses, comme les pipelines (conduites) et les interfaces des transports (p. ex. zones portuaires et gares de triage des chemins de fer).

Les Principes directeurs ne portent pas spécifiquement sur l'ensemble du domaine du transport de marchandises dangereuses comme tel (p. ex. transport routier, ferroviaire, maritime ou aérien). Cependant, un grand nombre des éléments considérés dans cette publication sont pertinents à la prévention, à la préparation et à l'intervention en matière d'accidents de transport et pourraient donc se révéler très utiles à ceux qui sont engagés dans les activités du secteur des transports. 
Les Principes directeurs sont fondés sur l'hypothèse voulant que toutes les installations de substances dangereuses soient conformes aux mêmes objectifs généraux de sécurité - c.-à-d. présentent la même probabilité de sécurité - quels que soient leur taille, leur emplacement et le type de propriété, soit public ou privé. De plus, l'orientation donnée ici s'applique non seulement aux installations où des substances chimiques sont produites ou mises au point, mais également à d'autres industries qui utilisent ou manipulent des produits chimiques potentiellement dangereux pour leurs opérations, ainsi qu'aux installations d'entreposage de produits chimiques.

Les Principes directeurs ont été élaborés de façon à pouvoir s'appliquer avec souplesse et permettre aux utilisateurs de choisir les clauses pertinentes et les adapter en fonction de leurs conditions particulières, comme la culture locale, le contexte juridique, la nature des risques, ainsi que l'importance et le type de ressources disponibles. À cet égard, il faut bien réaliser que toutes les clauses ne s'appliquent pas dans tous les cas.

La présente publication est le résultat d'un travail en collaboration - sous la supervision $d u$ Groupe de travail de l'OCDE sur les accidents chimiques - réalisé par un grand nombre d'experts de nombreux pays et organisations, dans les secteurs tant public que privé. Grâce à l'expérience collective de ce groupe diversifié d'experts internationaux, les Principes directeurs visent à mettre au point la «meilleure pratique ».

La publication cherche également à établir un équilibre dans l'orientation donnée, qui doit être générale et flexible - de façon à être utile à une vaste gamme d'entreprises, d'organismes et de collectivités - tout en se révélant suffisamment spécifique et détaillée pour servir de ressource aux décideurs.

Les Principes directeurs sont compatibles avec les autres outils de réglementation et matériels $\mathrm{d}^{\prime}$ orientation internationaux concernant la prévention, la préparation et l'intervention en matière d'accidents chimiques. Il s'agit d'outils et de matériels d'orientation élaborés dans le cadre et à l'aide de la Directive «Seveso II » de l'Union européenne ${ }^{6}$, du programme APELL7 du PNUE (Programme des Nations Unies pour l'environnement), des conventions et recommandations ${ }^{8}$ de l'Organisation $^{\prime}$ internationale du travail (OIT), des conventions ${ }^{9}$ de la Commission économique des Nations Unies pour l'Europe (CEE-ONU), de l'Organisation mondiale de la santé (OMS), du Programme international sur la sécurité des substances chimiques (PISSC) et enfin de l'Organisation maritime internationale $(\mathrm{OMI})^{10}$.

Il s'agit de la seconde édition des Principes directeurs. La première édition, publiée en 1992, a été largement diffusée dans le monde ${ }^{11}$. L'OCDE, ses pays membres et de nombreuses organisations non gouvernementales (y compris des organisations syndicales et des associations industrielles) en ont fait circuler des milliers de copies. De plus, d'autres organisations internationales, notamment le PNUE et la CEE-ONU, ont distribué des copies dans le cadre de leurs activités liées à des accidents chimiques ou industriels. Les commentaires reçus par le Secrétariat de l'OCDE montrent que les Principes directeurs se sont révélés très utiles pour l'élaboration et l'application des lois, règlements, politiques et pratiques.

La seconde édition des Principes directeurs a été mise à jour pour :

" tenir compte de l'expérience à l'échelle tant nationale qu'internationale, ainsi que des progrès au niveau des techniques et des politiques depuis 1992;

" incorporer les résultats des ateliers de l'OCDE et des examens spéciaux concernant diverses questions ${ }^{12}$, organisés au cours des dix dernières années et qui ont réuni une vaste gamme d'experts représentant divers intérêts et nationalités;

" élargir leur portée, de façon à inclure les interfaces des transports (p. ex. les zones portuaires et les gares de triage des chemins de fer) et les pipelines. 
Une différence importante par rapport à la première édition est l'inclusion des "Règles d'or ", qui reprennent les points importants des Principes directeurs.

De plus, cette version a été réorganisée en cinq parties importantes :

" Prévention des accidents chimiques

" Préparation aux situations d'urgence et atténuation

" Intervention en cas d'urgence

" Suivi des incidents

" Questions spéciales (comprenant deux sous-sections : "Questions transfrontalières/internationales » et «Installations fixes et transport »)

L'ordre d'apparition des clauses à l'intérieur de chaque partie a été changé de façon à mettre au premier plan le rôle de l'industrie, suivi de celui des pouvoirs publics, pour refléter le fait que l'industrie joue le premier rôle en ce qui a trait à la sécurité des installations.

La seconde édition comporte également une section révisée et mise à jour, soit l'« Explication des termes utilisés » (auparavant appelée glossaire). Cette section a été élaborée aux fins des Principes directeurs seulement; il ne s'agit donc pas de définitions acceptées pour tout autre usage. Pour éviter toute confusion, il est important pour la lecture du document de consulter cette section.

Enfin, le format de la seconde édition a été modifié pour rendre la publication plus conviviale.

\section{Objectif}

L'objectif de ces Principes directeurs de l'OCDE pour la prévention, la préparation et l'intervention en matière d'accidents chimiques est de déterminer des orientations générales, applicables à l'échelle mondiale, afin d'aider les divers intervenants à prendre les mesures appropriées pour prévenir les accidents mettant en cause des substances dangereuses et atténuer les impacts nuisibles des accidents, qui se produiront de toute façon.

Les expériences récentes, y compris les accidents majeurs dans des pays disposant d'une réglementation élaborée et d'un cadre administratif adéquat, ont montré que la législation et la réglementation, bien que nécessaires, ne sont pas suffisants pour prévenir les accidents ou préparer aux situations d'urgence. Il est donc important que toutes les parties prenantes s'engagent dans des initiatives complémentaires et s'inspirent mutuellement de l'expérience acquise.

\section{Portée}

La présente publication traite des nombreuses questions qu'il faut examiner si on veut assurer efficacement la sécurité dans le secteur chimique, c.-à-d. les mesures qui doivent être prises par l'industrie (y compris les employés), les pouvoirs publics, les communautés et d'autres intervenants afin : de réduire au minimum la probabilité qu'un accident se produise (prévention); d'atténuer les conséquences des accidents grâce à la planification pour les situations d'urgence, à l'aménagement du territoire et à la communication sur les risques (préparation/atténuation); enfin, de limiter, en cas d'accident, les conséquences nuisibles sur la santé, l'environnement et les biens (intervention). La publication comprend également les mesures qui doivent être prises pour tirer parti de l'expérience acquise lors d'accidents antérieurs et d'événements imprévus (suivi), ce qui permettra d'atténuer les incidents futurs (prévention). 
Il est souvent difficile de délimiter clairement quelles situations et mesures se situent à chacune de ces étapes, et on note beaucoup de chevauchement entre elles. Voilà pourquoi l'ensemble du processus est parfois décrit comme le «continuum de la sécurité » ou la "gestion cyclique de l'urgence » (voir ci-dessous).

\section{Continuum de la sécurité}



\section{Parties visées}

La présente publication donne des orientations à l'intention des divers groupes, personnes ou organisations qui sont concernés, intéressés ou potentiellement affectés par la prévention, la préparation et l'intervention en matière d'accidents chimiques (ils sont appelés ici «parties prenantes » ou « intervenants »).

\section{Industrie}

Étant donné que la responsabilité première de la sécurité des installations dangereuses incombe à ceux qui possèdent et exploitent ces dernières, la majeure partie de la présente publication porte sur la détermination des rôles et des responsabilités de l'industrie.

Par industrie, on entend les propriétaires/intervenants/exploitants des entreprises concernées (qu'elles soient privées ou publiques), la direction, les autres employés et les contractants travaillant pour l'installation. Dans la présente publication, le terme "direction » désigne toute personne qui prend des décisions pour l'entreprise, notamment les propriétaires et les directeurs; le terme "employé » désigne toute personne travaillant à ou pour une installation dangereuse, cela comprenant aussi bien la direction que les travailleurs et les contractants; par "travailleur ", on entend toute personne travaillant à ou pour une installation dangereuse, qui ne fait pas partie de la direction.

Aux fins de la présente publication, les organismes gouvernementaux qui exploitent des installations dangereuses (p.ex. les stations de traitement des eaux usées, les interfaces des transports, les entrepôts de produits chimiques) sont considérés comme faisant partie de $l^{\prime} \ll$ industrie ».

\section{Pouvoirs publics}

Les Principes directeurs reconnaissent l'importance des pouvoirs publics à toutes les étapes du continuum de la sécurité. La présente publication donne donc des orientations pour le rôle et les responsabilités des pouvoirs publics à tous les niveaux où il y a un engagement pour les nombreuses 
disciplines inhérentes à la prévention, à la préparation et à l'intervention en matière d'accidents chimiques (p. ex. environnement, santé publique, santé et sécurité professionnelles, protection civile, développement industriel, relations internationales). Ces orientations intéressent les autorités responsables de la réglementation et de son application (aux niveaux national, régional et local), le personnel d'intervention en cas d'urgence, les services de santé publique, les dispensateurs de soins médicaux et d'autres types d'organismes gouvernementaux.

\section{Collectivités et public}

Les Principes directeurs se penchent également sur le rôle du public, aussi bien le public en général que les membres des collectivités locales vivant au voisinage d'une installation dangereuse, ainsi que ceux qui peuvent être touchés si un accident survient. Il sont surtout axés sur la communication et la disponibilité de l'information concernant l'installation dangereuse, ainsi que sur la préparation en vue d'une situation d'urgence et l'intervention en cas d'urgence, et enfin sur la participation du public dans les décisions concernant les installations dangereuses. Condition sous-jacente : il devrait $\mathrm{y}$ avoir des canaux d'information et de communication à deux sens, de telle façon que les membres des collectivités ou du public non seulement reçoivent de l'information, mais qu'ils aient aussi la possibilité d'informer et d'exercer une certaine influence sur l'industrie, les pouvoirs publics et d'autres intervenants. On sait que la façon dont le public est informé et peut participer à la prise de décisions diffère d'un pays à l'autre et d'une communauté à l'autre, mais, certains principes sous-jacents doivent se retrouver partout.

\section{Autres intervenants}

La présente publication contient également des clauses concernant d'autres intervenants, comme les organisations syndicales, d'autres organisations non gouvernementales, des établissements de recherche (dont des établissements universitaires), et d'autres organisations intergouvernementales.

\section{Coopération et communication}

Un point souligné tout au long des Principes directeurs est l'importance de la coopération et de la communication parmi et entre les intervenants, notamment la coopération entre les intervenants de la collectivité où sont situées des installations dangereuses (autrement dit, au niveau local). Par exemple, pour que toutes les parties prenantes disposent de l'information dont elles ont besoin pour s'acquitter de leurs tâches, il doit y avoir une communication efficace entre les pouvoirs publics et l'industrie, entre les pouvoirs publics et le public, entre la direction et les employés, entre les différentes entreprises qui ont les mêmes intérêts (soit en raison de leur situation géographique, soit parce qu'elles sont confrontées aux mêmes problèmes), et enfin entre l'industrie et le public. La coopération non seulement renforce les capacités des intervenants, mais aide également à bâtir et à garder la confiance tout en évitant la confusion, les chevauchements, les conflits et les carences. 


\section{Intervenants concernés par les Principes directeurs}

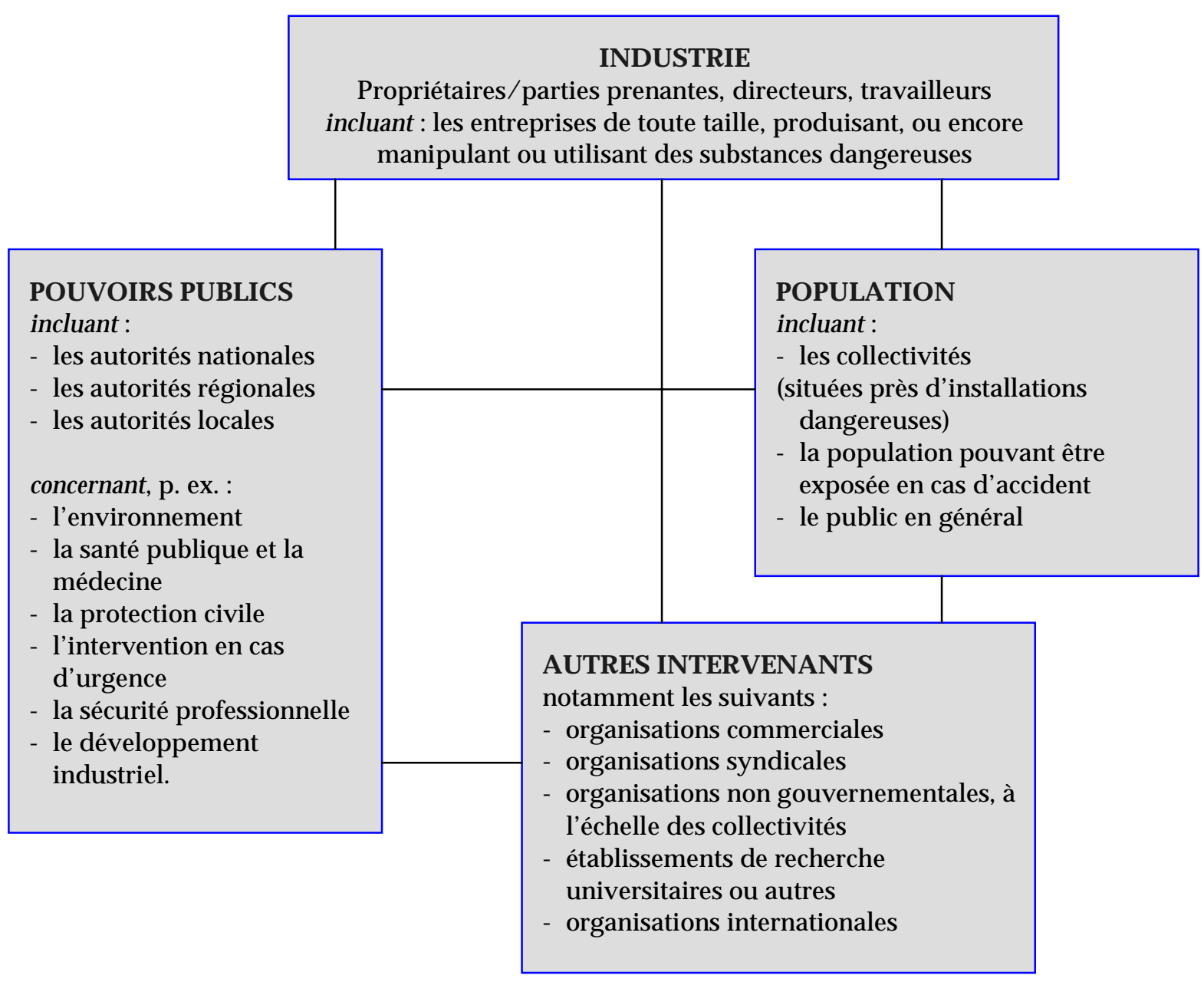

\section{Installations couvertes}

Les Principes directeurs s'appliquent à tous les types d'installations dangereuses - quels que soient leur taille ou leur emplacement, ou encore que leur propriétaire ou leur exploitant soit le secteur public ou privé - qui présentent un risque d'accident mettant en cause des substances dangereuses, avec des impacts sur la santé, l'environnement ou les biens. Ainsi, ces Principes directeurs s'appliquent à tout équipement ou installation fixe, qui produit, transforme, utilise, manipule, entrepose, transporte ou élimine des substances dangereuses ${ }^{13}$, et où il y a risque d'incendie, d'émission de produits toxiques, d'explosion, de déversement accidentel ou d'autres types d'accidents mettant en cause ces substances. Cela comprend par exemple :

" les producteurs et formulateurs de substances chimiques;

" les compagnies qui utilisent des substances dangereuses pour la préparation ou la transformation d'autres produits;

" les installations d'entreposage qui contiennent des substances dangereuses; 
" les interfaces des transports ${ }^{14}$, où des substances dangereuses sont chargées ou déchargées, ou encore transférées d'un véhicule à un autre (p. ex. trains, camions, navires), et les pipelines (ou conduites diverses).

Ainsi, le facteur déterminant pour l'application de ces notes d'orientation n'est pas la taille ou l'emplacement de l'installation, mais la possibilité qu'un accident mettant en cause des substances dangereuses puisse se produire, avec des impacts sur la santé, l'environnement ou les biens. À cet égard, les petites et moyennes entreprises ${ }^{15}$ ainsi que des sociétés qui ne font pas partie du secteur chimique doivent être conscientes de leurs responsabilités en matière de sécurité chimique; il faut néanmoins admettre que ce type d'établissement n'a pas toujours accès à du personnel spécialisé ni à l'information ou aux ressources dans ce domaine.

Ces notes d'orientation ne visent pas de façon générale le transport des substances dangereuses ${ }^{16}$; cependant, elles portent sur certains aspects du transport concernant les installations fixes, notamment :

" le transport soumis au contrôle des installations de substances dangereuses (p. ex. le transport sur place de ces substances et les décisions concernant la façon dont ces substances sont transportées vers et hors de l'installation);

" les interfaces des transports, notamment les gares de triage des chemins de fer et les zones portuaires; et,

" les pipelines.

Même si les accidents mettant en cause le transport de substances dangereuses par route, chemin de fer ou eaux navigables ne se situent pas spécifiquement dans le cadre de la présente publication, de nombreuses clauses des Principes directeurs s'appliquent à ces situations, notamment en ce qui concerne la préparation aux accidents lors du transport et à l'intervention.

\section{Aspects examinés}

Le but des Principes directeurs est de couvrir la vaste gamme de questions liées à la prévention, à la préparation et à l'intervention en matière d'accidents chimiques, incluant la production de rapports et les activités de suivi (mais excluant le nettoyage à long terme et la remise en état après un accident). Aux fins de la présente publication, un accident chimique est défini comme étant tout événement imprévu où il y a présence de substances dangereuses, qui cause ou peut causer des effets nocifs sur la santé, l'environnement ou les biens, comme la perte de l'enveloppe de confinement des substances dangereuses, les explosions et les incendies. Ces événements sont généralement le résultat de défaillances technologiques et (ou) d'erreurs humaines (ou une combinaison des deux). Le présent document s'applique également aux accidents chimiques causés par des désastres naturels, ou à certaines émissions soudaines, attribuables à des activités délibérées, p. ex. sabotage, terrorisme, vandalisme ou vol ${ }^{17}$.

Les accidents où il y a des émissions de matières radioactives n'ont pas été abordés dans la présente publication, vu que ce secteur est déjà traité par d'autres documents d'orientation de portée internationale. Les Principes directeurs ne traitent pas non plus des rejets de matière biologique. Cependant, ils étudient les accidents mettant en cause des substances chimiques qui ont été produites à des installations nucléaires ou par des processus biologiques. De plus, nombre des principes décrits dans la présente publication s'appliquent également dans le contexte des installations nucléaires et biotechnologiques ${ }^{18}$.

Les Principes directeurs n'abordent pas les événements à long terme, comme la pollution chronique par les substances chimiques. Cependant, les questions examinées ici sont étroitement liées 
à d'autres aspects de la protection environnementale, de la santé publique et professionnelle, et du développement durable. Il est donc avantageux pour les entreprises d'intégrer et de coordonner leurs programmes et leurs politiques concernant les divers aspects liés à la sécurité, à la santé et à l'environnement.

\section{Application à l'échelle mondiale}

Les Principes directeurs ont été conçus pour être applicables et utiles à l'échelle mondiale, et pas seulement dans les pays de l'OCDE. Ces notes d'orientation ont donc été conçues de façon à être conformes et complémentaires à d'autres documents internationaux d'orientation, et ce, après consultation d'un grand nombre d'organisations publiques et privées lors du processus d'élaboration et d'examen.

Comme dans le cas de la première édition, on espère que les Principes directeurs seront diffusés dans le monde entier par l'intermédiaire de l'OCDE et de ses pays membres, d'organisations non gouvernementales intéressées et de diverses autres organisations internationales ou de l'ONU. On encourage toutes les parties intéressées à faire connaître les Principes directeurs dans leurs pays et organisations et à adapter le texte à leurs besoins et conditions spécifiques.

\section{DOCUMENT D'ORIENTATION SUR LES INDICATEURS DU NIVEAU DE SÉCURITÉ}

Un ouvrage connexe, intitulé Document d'orientation sur les indicateurs de performance en matière de sécurité (2003) sera publié immédiatement après la 2e édition des Principes directeurs. Le document d'orientation doit aider les intervenants concernés à élaborer un programme pour évaluer leur propre niveau de prévention, de préparation et d'intervention en matière d'accidents chimiques. Cela devrait améliorer la capacité des entreprises industrielles, des pouvoirs publics et des organisations communautaires intéressés à déterminer si les nombreuses étapes prises pour réduire les risques d'accident et améliorer les mesures de préparation et d'intervention permettent réellement de rendre les collectivités plus sûres et de réduire les risques pour la santé humaine et l'environnement.

Le document d'orientation ne définit pas une méthodologie précise, mais donne plutôt des indications sur la façon d'élaborer des programmes IPS, ainsi que des éléments qui pourraient être employés dans ces programmes d'après l'expérience collective des experts dans ce domaine.

Le document d'orientation est conçu comme un outil offrant une grande souplesse et pouvant être utilisé à titre volontaire par les intervenants partout dans le monde. Il est destiné à compléter d'autres activités connexes, y compris les initiatives du secteur industriel. 


\section{LES « RÈGLES D’OR »}

Les "règles d'or " sont une nouveauté de la 2ème édition des Principes directeurs. Elles visent à mettre en exergue, en quelques pages, le rôle et les responsabilités des principales parties prenantes dans la prévention, la préparation et l'intervention en matière d'accidents chimiques. Il convient de noter que ces règles renvoient aux meilleures pratiques, c'est-à-dire à des objectifs destinés à être atteints progressivement. Il s'agit non d'actions ponctuelles, mais de principes qui exigent une vigilance de tous les instants. Cette section explique brièvement chacune des règles d'or. L'annexe VI résume ces dernières.

Les règles d'or ne constituent pas une synthèse complète des Principes directeurs; elles n'abordent pas l'intégralité des questions traitées dans cet ouvrage. Pour bien saisir leurs tenants et leurs aboutissants, il est important de se reporter au texte complet des Principes directeurs.

\section{RÔLE DE TOUTES LES PARTIES PRENANTES}

" Pour protéger la santé, l'environnement et les biens, considérez prioritairement la réduction des risques et la prévention des accidents chimiques, ainsi que la préparation aux situations $d^{\prime}$ urgence et l'intervention en cas d'accident.

Si les risques d'accident se situent d'abord dans les collectivités où se trouvent des installations dangereuses, et nécessitent donc des mesures au niveau local, les intervenants ont également des responsabilités à assumer aux niveaux régional, national et international.

" Communiquez et coopérez avec les autres parties prenantes sur tous les aspects de la prévention, de la préparation et de l'intervention en matière d'accidents chimiques.

La communication et la coopération devraient se fonder sur une politique d'ouverture, ainsi que sur l'objectif partagé de réduction de la probabilité d'accidents et d'atténuation de leurs effets dommageables. Aspect important, la population pouvant être touchée devrait disposer de l'information nécessaire pour atteindre les objectifs de prévention et de préparation, et avoir l'occasion de participer, le cas échéant, à la prise de décisions concernant les installations dangereuses.

\section{RÔLE DE L'INDUSTRIE (employeurs et travailleurs)}

\section{Employeurs}

" Soyez au fait des dangers et des risques présents aux installations abritant des substances dangereuses.

Toutes les entreprises qui produisent, utilisent, entreposent ou manipulent d'une façon ou d'une autre des substances dangereuses devraient entreprendre, en coopération avec les autres parties prenantes, les travaux d'identification des dangers et d'évaluation des risques, qui sont nécessaires pour acquérir une connaissance complète des menaces pour les employés, la population, l'environnement et les biens en cas d'accident. Ces travaux devraient intervenir dès les toutes premières phases de conception et de construction et se poursuivre tout au long de l'exploitation et dans le cadre de la maintenance des installations; ils devraient tenir compte des possibilités d'erreur humaine et de défaillance technologique, ainsi que des possibilités de rejets provoqués par des catastrophes naturelles et des actes délibérés (par exemple, terrorisme, sabotage, vandalisme ou vol). Les évaluations devraient être réitérées périodiquement et à chaque fois que l'installation subit une modification notable. 
" Faites prévaloir une « culture de la sécurité » connue et acceptée dans l'entreprise tout entière. La culture de la sécurité, qui doit se retrouver dans la politique définie par l'entreprise en la matière, renvoie tout à la fois à une attitude qui consiste à faire de la sécurité une priorité (en partant du principe que les accidents sont évitables) et à l'existence d'une infrastructure idoine (englobant, par exemple, les politiques et les procédures). Pour qu'elle soit efficace, il faut que la direction de l'entreprise s'engage ostensiblement en faveur de la sécurité et que les employés ${ }^{20}$ et leurs représentants y adhèrent et y participent.

" Établissez des systèmes de gestion de la sécurité et surveillez/examinez leur application.

Dans les installations dangereuses, les systèmes de gestion de la sécurité impliquent notamment le recours à des technologies et procédés adaptés et la mise en place d'une véritable structure d'organisation (procédures et pratiques opérationnelles, programmes de formation pratique et théorique, efficace, présence de personnel bien formé aux niveaux appropriés, affectation des ressources nécessaires, etc.). Ce sont là autant de facteurs qui contribuent à réduire les dangers et les risques. Pour s'assurer que ces systèmes sont adéquats, il est primordial d'en faire le suivi au moyen de dispositifs d'examen adaptés et efficaces (y compris au niveau des politiques, des procédures et des pratiques).

" Appliquez des principes technologiques à plus grande "sécurité intrinsèque" dans la conception et l'exploitation des installations dangereuses.

Cela devrait permettre de réduire la probabilité d'accidents et d'atténuer au maximum les conséquences de tout accident éventuel. Par exemple, les possibilités suivantes devraient être considérées, dans la mesure où elles ont pour effet de diminuer les risques : limiter autant que possible la quantité de substances dangereuses utilisée; remplacer les substances dangereuses par d'autres qui le sont moins; abaisser les pressions et/ou les températures de fonctionnement; améliorer la gestion des stocks; enfin, employer des procédés plus simples. Ces mesures pourraient être complétées par le recours à des systèmes de secours.

" Apportez un soin particulier à la gestion du changement.

Tout changement significatif (de techniques de production, de personnel et de procédures, par exemple) a pour effet d'accroître le risque d'accident, et il en va de même pour les opérations de maintenance, de réparation, de démarrage et d'arrêt d'exploitation. Il est particulièrement important d'en être conscient et de prendre des mesures de sécurité appropriées lorsque l'on prévoit procéder à des changements significatifs - et ce avant d'appliquer les changements.

4 Préparez-vous à tout accident susceptible de se produire.

Il faut être conscient du fait qu'on ne peut pas éliminer totalement tout risque d'accident. C'est pourquoi il est primordial de planifier des mesures d'urgence adaptées pour réduire au minimum la probabilité et l'ampleur d'éventuels effets néfastes sur la santé, l'environnement ou les biens. La planification des mesures d'urgence doit être réalisée à l'intérieur de l'installation et soutenue à l'extérieur (notamment par l'information des populations pouvant être touchées).

" Aidez les autres à assumer leur rôle et leurs responsabilités.

A cette fin, les employeurs devraient coopérer avec l'ensemble des employés et leurs représentants, les pouvoirs publics, les collectivités locales et d'autres membres du public. En outre, ils devraient s'efforcer d'aider les autres entreprises (notamment leurs fournisseurs et clients) à respecter les normes de sécurité idoines. Par exemple, les fabricants de substances dangereuses devraient mettre en application un programme efficace de gérance des produits.

" Recherchez en permanence une amélioration continue.

S'il n'est pas possible d'éliminer tous les risques d'accident dans les installations dangereuses, l'objectif devrait être de trouver les moyens d'améliorer les technologies, les systèmes de gestion 
et les qualifications du personnel afin de se rapprocher de l'idéal «zéro accident ». Dans cette optique, les employeurs devraient s'attacher à tirer tous les enseignements des accidents survenus et évités de justesse dans leur entreprise et ailleurs.

\section{Travailleurs}

" Agissez conformément à la culture de la sécurité de l'entreprise, à ses procédures de sécurité et à la formation reçue.

Dans l'accomplissement de leur tâche, les travailleurs devraient respecter l'ensemble des procédures et pratiques relatives à la prévention, à la préparation et à l'intervention en matière d'accidents, conformément à la formation et aux instructions reçues de leur employeur. Tous les employés (y compris les sous-traitants) devraient signaler à leur supérieur hiérarchique toute situation pouvant à leurs yeux représenter un risque significatif.

" Ne ménagez aucun effort pour vous informer et communiquer à la direction les renseignements et toute information en retour.

Il est important que tous les employés, y compris les contractants, connaissent les risques qui existent dans l'entreprise dans laquelle ils travaillent, et qu'ils comprennent comment ils peuvent éviter de les amplifier ou d'en créer de nouveaux. Dans la mesure du possible, les travailleurs devraient faire remonter l'information sur les questions de sécurité jusqu'à la direction. À cet égard, les travailleurs et leurs représentants devraient coopérer avec les employeurs à l'élaboration et à l'application de systèmes de gestion de la sécurité, avec notamment des procédures assurant une éducation, une formation et un perfectionnement adéquats des employés. Les travailleurs et leurs représentants devraient aussi avoir la possibilité de participer aux activités de surveillance et d'enquête conduites par l'employeur ou les autorités compétentes en liaison avec les mesures de prévention, de préparation et d'intervention en matière d'accidents chimiques.

" Prenez l'initiative de contribuer à informer et à sensibiliser votre communauté.

Bien informés et concernés, les employés d'une installation dangereuse peuvent jouer un rôle important en se faisant les ambassadeurs de la sécurité dans leur communauté.

\section{RÔLE DES POUVOIRS PUBLICS}

" Élaborez des politiques, des règlements et des procédures, veillez à les faire appliquer et employez-vous à les améliorer en permanence.

Il est important que les pouvoirs publics définissent des politiques, des règlements et des procédures, et qu'ils mettent en place des mécanismes pour veiller à leur application effective. Ils devraient aussi procéder régulièrement et selon les besoins au réexamen et à la mise à jour de ces politiques, règlements et procédures. Dans cette optique, les pouvoirs publics devraient se tenir au courant et tenir compte des évolutions pertinentes, notamment les changements intervenant dans les technologies et les pratiques des entreprises, l'évolution du niveau de risque dans la collectivité de leur ressort, l'expérience acquise dans le cadre de la mise en œuvre de la législation en vigueur, et les études de cas d'accident. Ils devraient associer les autres parties prenantes au processus de réexamen et de mise à jour.

" Fournissez le leadership afin de motiver toutes les parties prenantes à remplir leur rôle et à assumer leurs responsabilités.

À l'intérieur de leur domaine de compétence et d'influence, tous les pouvoirs publics devraient s'efforcer de motiver les autres parties prenantes à reconnaître l'importance de la prévention, de la préparation et de l'intervention en matière d'accidents, et à prendre les mesures qui s'imposent 
pour réduire au minimum les risques d'accident et atténuer les effets d'un éventuel accident. Dans cette optique, les pouvoirs publics devraient adopter des dispositifs réglementaires adaptés et les faire respecter, encourager des initiatives volontaires et mettre en place des mécanismes pour favoriser l'éducation et l'échange d'informations.

" Suivez de près l'industrie pour veiller à ce que les risques soient convenablement traités. Les pouvoirs publics devraient établir des mécanismes de surveillance des installations dangereuses, afin de veiller au respect de l'ensemble des lois et règlements applicables, ainsi qu'à l'existence et au bon fonctionnement des éléments nécessaires à un système de gestion de la sécurité, compte tenu de la nature des risques causés par les installations (y compris l'éventualité de rejets délibérés). Ils peuvent également profiter de l'occasion qu'offrent ces mécanismes de confronter les expériences avec les employés compétents des installations.

" Favorisez une communication et une coopération efficaces entre les parties prenantes.

L'information est un volet crucial des programmes relatifs à la sécurité. Les pouvoirs publics ont un rôle important à jouer en veillant à ce que les informations appropriées soient communiquées à toutes les parties concernées et reçues par celles-ci. Une mission particulière consiste à favoriser la sensibilisation de la population aux risques chimiques auxquels est exposée leur collectivité, afin que les membres du public soient rassurés quant aux mesures de sécurité en place, qu'ils sachent quoi faire en cas d'accident et qu'ils puissent participer de façon constructive aux processus décisionnels pertinents. Les pouvoirs publics sont également en mesure de faciliter la mise en commun des expériences (à l'intérieur et au-delà des frontières).

" Favorisez la coordination inter-institutions.

La prévention, la préparation et l'intervention en matière d'accidents chimiques constituent un domaine d'activité interdisciplinaire par nature, qui fait intervenir les autorités responsables de différents secteurs et se situe à différents niveaux. Pour assurer une prévention, une préparation et une intervention efficaces, et afin de garantir une utilisation rationnelle des ressources, il importe que tous les organismes concernés coordonnent leurs activités.

"Soyez au fait des risques relevant de votre domaine de compétence et planifiez en conséquence.

Les pouvoirs publics sont responsables de la planification d'urgence à l'extérieur des installations, compte tenu des plans pertinents établis dans les installations. Cette planification devrait intervenir en coordination avec les autres parties prenantes. En outre, les pouvoirs publics devraient veiller à la disponibilité des ressources nécessaires à une intervention (compétences, information, matériel, installations médicales, fonds, etc.).

" Atténuez les effets des accidents par des mesures d'intervention appropriées.

En cas d'accident ayant des conséquences à l'extérieur de l'installation concernée, les pouvoirs publics (souvent au niveau local) sont responsables au premier chef des interventions destinées à réduire au minimum le nombre de victimes et à protéger l'environnement et les biens.

"Prenez des mesures et des dispositions adaptées et cohérentes d'aménagement du territoire. L'aménagement du territoire (c'est-à-dire l'élaboration et la mise en oeuvre de plans de zonage généraux, avec les lieux d'implantation spécifiques des installations dangereuses et d'autres constructions) peut contribuer à faire en sorte que les installations soient situées de façon satisfaisante du point de vue de la protection de la santé, de l'environnement et des biens si un accident se produit. Les mesures et dispositions prises en la matière peuvent éviter l'implantation de quartiers nouveaux (logements, locaux commerciaux, édifices publics, etc.) à proximité d'installations dangereuses. Elles devraient aussi prévenir des modifications contre-indiquées dans les installations existantes (par exemple, mise en place d'équipements ou de procédés 
nouveaux). Enfin, les mesures et dispositions de planification de l'utilisation des sols devraient permettre d'exiger l'apport de modifications aux installations et constructions existantes afin de les rendre conformes aux normes de sécurité en vigueur.

\section{RÔLE DES AUTRES PARTIES PRENANTES (collectivités/particuliers)}

" Soyez conscient des risques auxquels est exposée votre collectivité et sachez quoi faire en cas d'accident.

Les particuliers vivant à proximité d'une installation dangereuse et ceux qui risquent d'être touchés en cas d'accident devraient s'assurer qu'ils savent à quels risques ils sont exposés et ce qu'ils doivent faire en cas d'accident pour atténuer les possibles effets néfastes sur la santé, l'environnement et les biens (connaître les signaux d'alarme, la conduite à tenir, etc.). Pour cela, il importe qu'ils lisent et conservent les documents d'information qu'ils reçoivent, partagent ces informations avec les autres membres du foyer et sollicitent au besoin des renseignements complémentaires.

4 Participez à la prise de décisions concernant les installations dangereuses.

Bien souvent, la législation en vigueur permet aux membres du public de participer à l'élaboration des décisions concernant les installations dangereuses, par exemple en donnant leur avis sur les projets de règlements et les décisions de zonage, ou en contribuant au processus conduisant à l'autorisation ou au choix du site de certaines installations. Les membres du public devraient saisir les occasions qui leur sont ainsi offertes de faire entendre la voix de la collectivité. Ils devraient œuvrer pour faire en sorte qu'il y ait, si nécessaire, cette possibilité, et que le public dispose des informations essentielles à une participation efficace.

" Coopérez avec les autorités locales et l'industrie aux interventions d'urgence et à leur planification

Les représentants de la collectivité devraient profiter des occasions qui leur sont offertes de contribuer au processus de planification des mesures d'urgence, à l'intérieur comme à l'extérieur des installations. En outre, les membres du public devraient coopérer dans le cadre des exercices organisés pour tester les plans d'urgence, en suivant les instructions et en faisant part aux autorités de toute information utile. 


\section{NOTES}

1. La Division environnement, santé et sécurité produit des publications dans huit domaines (Essais et évaluation; Principes de bonnes pratiques de laboratoire et vérification du respect de ces principes; Gestion du risque; Harmonisation de la surveillance réglementaire de la biotechnologie; Accidents chimiques; Inventaires des émissions et des transferts de matières polluantes; Sécurité des nouveaux aliments destinés à la consommation humaine et animale). Davantage de renseignements sur le Programme ESS et les publications ESS sont disponibles au site Internet de l'OCDE (www.oecd.org/ehs/).

2. Le terme "sécurité » utilisé dans la présente publication comprend la protection de la santé, de l'environnement et des biens en autant que ces domaines se situent dans le cadre de la prévention, de la préparation et de l'intervention en matière d'accidents mettant en cause des substances dangereuses.

3. Voir « Objectif » et « Portée » pour plus de détails sur les parties concernées.

4. Voir l'annexe I pour une explication des termes «accident » et «quasi-accident », utilisés dans la présente publication. Un «accident » est défini comme étant un événement imprévu, pouvant être causé, par exemple, par une erreur humaine ou technologique, un acte délibéré (sabotage, terrorisme, vandalisme ou vol) ou encore un désastre naturel. Un accident peut causer des effets nuisibles directs ou indirects sur l'environnement, la santé et (ou) les biens, par suite d'incendies, d'expositions ou de rejets de substances dangereuses. Un accident peut également entraîner des effets nuisibles indirects sur la santé par contamination notamment de l'eau ou des aliments.

5. On sait que l'aménagement du territoire peut être considéré comme une mesure préventive (p.ex. en veillant à ce que les installations dangereuses soient séparées des autres développements par une distance adéquate, ce qui empêcherait tout effet nuisible) ou comme un moyen d'atténuer les effets nocifs exercés par un accident.

6. Directive du Conseil 96/82/EC du 9 décembre sur le contrôle des principaux risques d'accident mettant en cause des substances dangereuses. (Note: une proposition visant à modifier la directive est à l'étude).

7. APELL est l'acronyme de «Awareness and Preparedness for Emergency at Local Level ». Pour plus de renseignements, voir l'encadré texte à la fin du chapitre 5.

8. Convention sur la prévention des accidents industriels majeurs ( $\left.\mathrm{n}^{\circ} 174\right)$ et recommandation connexe ( ${ }^{\circ} 181$ ) adoptées en 1993, et Convention concernant la sécurité dans l'utilisation des produits chimiques au travail (no 170) et recommandation connexe (no 177), adoptées en 1990.

9. Convention sur les effets transfrontières des accidents industriels et Convention sur la protection et l'utilisation des cours d'eau transfrontières et des lacs internationaux.

10. Beaucoup de ces documents sont énumérés à l'annexe IV; certains présentent des renseignements et des notes d'orientation supplémentaires sur les sujets abordés ici.

11. La première édition des Principes directeurs est disponible en plusieurs langues. L'information sur les traductions des première et seconde éditions est présentée sur le site Internet de l'OCDE: www.oecd.org/ehs/ 
12. Une liste de ces ateliers est incluse à l'annexe IV. Des copies des rapports de ces ateliers sont disponibles au site www.oecd.org/ehs/

13. Pour les besoins de la présente publication, une substance dangereuse est définie à l'annexe I («Explication des termes utilisés »). L'expression «substance dangereuse » est aussi utilisée sous des formes similaires dans d'autres documents internationaux, portant notamment sur les marchandises dangereuses, les cargaisons dangereuses ou encore les produits ou substances chimiques dangereux.

14. Les interfaces des transports comprennent, par exemple, les zones portuaires et les gares de triage des chemins de fer.

15. Voir l'encadré texte sur les PME à la fin du chapitre 1.

16. Dans le contexte des transports, on utilise souvent les expressions «marchandises dangereuses » ou «cargaisons dangereuses » pour décrire les produits transportés. Dans le présent document, on utilisera l'expression "substances dangereuses », comme équivalent général des expressions «marchandises dangereuses» ou «cargaisons dangereuses» telles qu'employées dans les ententes internationales (comme celles conclues par le Comité d'experts de l'ONU sur le transport des marchandises dangereuses, l'OMI ou la CEE-ONU), mais elle n'englobera pas les matières radioactives.

17. Le présent document ne traite pas des questions liées à des actes délibérés, bien que la plupart des notes d'orientation s'appliquent à de telles situations. Ces questions seront traitées ultérieurement.

18. Certains aspects des Principes directeurs ne s'appliquent pas aux installations militaires, du fait qu'ils pourraient représenter une menace pour la sécurité nationale.

19. Aux fins de la présente publication, on entend par partie prenante (ou intervenant) tout individu, groupe ou organisme qui participe à la prévention, à la préparation et à l'intervention en matière d'accidents chimiques, qui s'y intéresse ou qui pourrait en subir les effets. Cela comprend notamment les propriétaires et les directeurs d'installations dangereuses, les autres employés travaillant dans les installations et leurs représentants, les pouvoirs publics à tous les niveaux, ainsi que la population et les collectivités.

20. Aux fins de la présente publication, on entend par « employé » tout individu travaillant dans une installation dangereuse ou pour le compte de celle-ci. Cela comprend les employeurs, les travailleurs et les contractants.

21. Aux fins de cette publication, l'expression «pouvoirs publics» désigne les autorités nationales, régionales et locales chargées des différents aspects de la prévention, de la préparation et de l'intervention en matière d'accidents chimiques. Cela comprend notamment les organes chargés de la protection de l'environnement, de la santé publique, de la sécurité du travail, du secteur industriel, ainsi que des interventions d'urgence et de la protection civile. 
Partie A

PRÉVENTION DES ACCIDENTS CHIMIQUES

Cette partie est de loin la plus longue des Principes directeurs, reflétant ainsi l'importance accordée à la prévention des accidents chimiques. Le chapitre 1 (Principes généraux) comprend une vue d'ensemble des rôles et des responsabilités des divers intervenants concernés et reconnaît que beaucoup de parties aux intérêts divergents sont préoccupées par la question de la prévention. On insiste également sur la nécessité d'une étroite coopération entre les parties prenantes et sur le fait que les risques doivent être abordés à l'échelle locale. Ces points sont davantage développés aux chapitres 2 à 4 , lesquels donnent des renseignements plus spécifiques sur les rôles et les responsabilités respectives de l'industrie, des pouvoirs publics, de la population en général et des autres intervenants. 


\section{Chapitre 1}

\section{PRINCIPES GÉNÉRAUX}

1.1 Le principal objectif des programmes de sécurité aux installations dangereuses est la prévention des accidents entraînant des effets nuisibles sur la santé, l'environnement ou les biens (tout en reconnaissant que des accidents mettant en cause des substances dangereuses peuvent malgré tout se produire).

1.2 La prévention des accidents mettant en cause des substances dangereuses est au cœur des préoccupations de tous les intervenants, et notamment des suivants : l'industrie (p. ex. propriétaires et directeurs d'installations dangereuses, autres employés et contractants travaillant à ou pour ces installations, représentants ${ }^{1}$ des employés); pouvoirs publics aux échelles nationale, régionale et locale; collectivités.

" Pour que les activités de prévention des accidents soient efficaces, une grande collaboration est indispensable entre toutes les parties concernées et à tous les niveaux. À l'intérieur de collectivités où il y a présence d'installations dangereuses, il est important que l'industrie, les pouvoirs publics et la population en général travaillent ensemble pour réduire les risques d'accident.

" Cette coopération doit être fondée sur une politique de transparence, qui aidera notamment à convaincre le public que les mesures appropriées sont prises pour limiter le risque que des accidents mettant en cause des substances dangereuses exercent des effets nocifs à l'extérieur de l'installation.

1.3 Toutes les installations dangereuses devraient se conformer aux mêmes objectifs généraux de sécurité (c.-à-d. répondre aux mêmes attentes en matière de sécurité), peu importe leur taille, leur emplacement ou d'autres facteurs. L'obligation de l'industrie de fonctionner de façon sûre s'applique aux entreprises de toute taille, qui produisent, utilisent, manipulent, transportent, entreposent ou éliminent des substances dangereuses, y compris les entreprises qui ne sont pas des producteurs de substances chimiques ou qui ne sont pas considérées comme faisant partie du secteur chimique.

- 1.4 La sécurité doit être une partie intégrante des activités commerciales d'une entreprise, et toutes les installations dangereuses doivent tendre vers l'objectif ultime de zéro incident ${ }^{2}$. Toutes les ressources doivent être consacrées pour atteindre ce but.

" L'établissement d'un objectif ultime de zéro incident constitue un incitatif pour atteindre le rendement optimal et persévérer dans la quête d'une plus grande sécurité.

" La progression vers ce but peut être facilitée en établissant des objectifs de communication et de publication associés à la sécurité et en mesurant la progression vers ces objectifs.

1.5 La direction des installations dangereuses a la responsabilité première du fonctionnement sûr des installations et de la recherche de moyens pour y arriver.

1.6 L'industrie devrait contrôler régulièrement et évaluer la performance en matière de sécurité des installations dangereuses afin : 
- d'évaluer les réalisations par rapport aux objectifs fixés;

- de déterminer si les politiques et les décisions spécifiques, axées sur la sécurité, ont été efficacement mises en pratique;

- de concentrer les ressources là où on en a le plus besoin;

- de fournir l'information voulue pour justifier l'ajustement ou le redressement des objectifs et réaliser d'autres améliorations;

- de démontrer l'engagement de la direction en faveur de la sécurité et favoriser le processus d'amélioration;

- d'établir une base permettant de déterminer si une performance est bonne ou insuffisante;

- de fournir l'information appropriée sur les réalisations en matière de sécurité aux pouvoirs publics, à la collectivité, aux intervenants et aux organisations non gouvernementales (ONG); et

- de contribuer aux activités de formation pratique et théorique.

1.7 Lorsque certaines activités sont confiées à des tierces parties, la direction doit s'assurer que les contrats contiennent des clauses sur les rôles et les responsabilités concernant la sécurité et prévoient suffisamment de ressources pour permettre à ces parties d'assumer ces rôles et responsabilités.

" Des procédures doivent être en place pour déterminer si les contractants (tierces parties) ont la capacité d'assumer leurs tâches en respectant les normes de sécurité.

" Dans tous les cas, c'est la direction qui assume la responsabilité de la sécurité des installations.

1.8 La direction devrait coopérer avec les pouvoirs publics pour aider ceux-ci à s'acquitter de leurs responsabilités.

1.9 La direction devrait encourager et faciliter la déclaration par tous les employés (incluant les contractants) de tout accident ou quasi-accident chimiques, de façon à pouvoir apprendre de l'expérience du passé. Cette façon de faire est indispensable si on veut continuer à améliorer la sécurité.

1.10 Il incombe aux producteurs de substances dangereuses de promouvoir la sécurité dans la gestion de ces substances tout au long de leur cycle de vie, et de fournir de l'aide aux utilisateurs en aval, le tout en conformité avec le principe de la gérance des produits.

1.11 Tous les employés ${ }^{3}$ devraient partager la responsabilité et avoir un rôle à jouer en matière de prévention des accidents, et, à cette fin, devraient assumer leurs tâches en veillant activement à la sécurité, aider les autres à en faire autant, et enfin contribuer à la mise au point et à l'application des politiques et des pratiques concernant la sécurité.

1.12 Les pouvoirs publics devraient fixer des objectifs généraux en matière de sécurité, établir un cadre de contrôle clair et cohérent et s'assurer, grâce à des mesures appropriées $\mathrm{d}$ 'inspection et de vérification, que toutes les exigences sont respectées.

" Ils devraient être proactifs en favorisant la recherche de nouvelles méthodes pour la prévention des accidents en plus de leur rôle plus traditionnel consistant à réagir suite à des craintes particulières de la population. 
" Ils devraient prendre des initiatives afin de sensibiliser tous les secteurs de la société à la nécessité de prévenir les accidents, de déterminer les outils requis, et de susciter une culture nationale visant à promouvoir la prévention des accidents.

4 Ils devraient s'assurer que le public est informé au sujet des installations dangereuses et l'aider à bien comprendre cette information, ce qui permettra de gagner sa confiance à l'égard de la réglementation en vigueur.

1.13 Les pouvoirs publics devraient coopérer avec l'industrie (direction et autres employés) et encourager celle-ci à assumer ses responsabilités, c.-à-d. à veiller au fonctionnement sûr de ses installations dangereuses et à informer le public au sujet des risques et des mesures de sécurité. De plus, les pouvoirs publics devraient encourager toute aide permettant d'améliorer les programmes de sécurité dans les petites et moyennes entreprises (PME) et dans d'autres entreprises dont les ressources et le personnel sont limités.

1.14 Les pouvoirs publics devraient, dans le cadre de leur programme d'application de la loi, vérifier régulièrement la performance des installations dangereuses. Les programmes d'inspection permettent également aux pouvoirs publics de partager l'information au sujet de la sécurité avec la direction de ces installations, et de les aider à établir des priorités pour leur travail.

1.15 Les pouvoirs publics devraient conclure des arrangements communs avec les entreprises de leur collectivité afin d'améliorer la sécurité chimique en général, et notamment d'aider les PME et d'autres entreprises disposant de ressources limitées à prévenir les accidents mettant en cause des substances dangereuses et à se préparer aux situations d'urgence.

1.16 Des groupes d'intervenants devraient être constitués aux échelles régionale et nationale comme moyens d'obtention et de diffusion de l'information sur la sécurité, ainsi que pour la mise au point $\mathrm{d}^{\prime}$ approches facilement acceptables par toutes les parties.

1.17 Les pouvoirs publics, les associations industrielles et autres devraient améliorer le partage de l'information et des documents d'orientation concernant la prévention des accidents chimiques et, de façon générale, améliorer la performance des installations dangereuses en matière de santé, de sécurité et d'environnement. Cette information devrait porter aussi bien sur les éléments techniques que sur les questions liées à des facteurs humains et à des systèmes de gestion de la sécurité.

1.18 Tous les intervenants devraient prendre part à l'enjeu fondamental que constitue l'acceptabilité-tolérabilité du risque dans une collectivité.

" À cet égard, chaque pays ou organisation devrait choisir ses propres critères d'acceptabilité-tolérabilité. Un cadre pour juger les critères, accepté par tous, facilitera l'obtention d'un consensus sur ce qui est acceptable-tolérable.

" L'évaluation du risque permet d'éclairer le processus décisionnel, mais elle ne devrait pas constituer le seul facteur décisif. Ce type de question se situe également au niveau du jugement socio-politique. 
1.19 Des moyens devraient être mis à la disposition des entreprises aux ressources limitées, qui ont besoin d'aide ou d'assistance pour améliorer leurs programmes de sécurité (notamment certaines PME).

" Une approche polyvalente, comptant une grande variété d'entités et de programmes, pourrait être nécessaire pour faire face aux possibles préoccupations et limitations des PME et d'autres entreprises qui ont besoin d'aide ou d'assistance.

" Ce type d'entreprise devrait, par exemple, envisager les démarches suivantes:

- conclure des arrangements de coopération avec les pouvoirs publics, les associations industrielles ou commerciales et (ou) d'autres entreprises afin d'améliorer la sécurité dans leurs communautés et d'obtenir les renseignements et la formation nécessaires pour une meilleure sécurité;

- former des partenariats avec d'autres entreprises pour constituer des groupes d'intervention comptant sur une "aide mutuelle », de façon à rassembler et à partager les ressources limitées en cas d'accident chimique (pour former ces groupes d'aide mutuelle, les PME concernées devraient demander l'aide des pouvoirs publics);

- bâtir des relations mutuellement avantageuses avec les fournisseurs et les clients, et tirer parti de leurs offres d'aide et de vérification;

- rejoindre les rangs d'organisations professionnelles.

" Les divers intervenants (entreprises individuelles, associations industrielles ou commerciales, partenaires industrie-gouvernement, pouvoirs publics, commissions de sécurité, organisations professionnelles, organisations ou confédérations syndicales, établissements de recherche ou d'enseignement, médias) devraient fournir de l'aide, de l'information, des outils techniques et des incitatifs aux entreprises qui ont besoin d'assistance. Par exemple, dans leur collectivité, les grosses entreprises devraient mettre leur expertise en sécurité chimique à la disposition des entreprises plus petites. Les entreprises devraient également faire leur possible pour fournir de l'aide aux clients, aux fournisseurs et aux autres entreprises d'industries apparentées. 


\section{PETITES ET MOYENNES ENTREPRISES}

Toutes les installations dangereuses doivent se conformer aux mêmes objectifs généraux de sécurité (c.-à-d. répondre aux mêmes attentes en matière de sécurité), peu importe leur taille, leur emplacement ou d'autres facteurs.

Les PME partagent généralement un certain nombre de caractéristiques qui suscitent des craintes au sujet des risques d'accident. Par exemple, de façon générale, elles possèdent une structure de fonctionnement moins normative que les grandes entreprises, elles emploient peu d'ingénieurs et de spécialistes en sécurité, et enfin comptent sur leurs partenaires commerciaux et sur d'autres sources externes pour l'information concernant la sécurité chimique.

D'un autre côté, les PME comptent beaucoup de points forts pouvant être exploités pour améliorer la gestion de la sécurité chimique. Par exemple, elles peuvent modifier leurs procédures plus rapidement que les grosses compagnies et il y a de plus fortes chances qu'elles disposent de canaux de communication ouverts.

Les PME sont examinées dans les Principes directeurs pour les raisons suivantes: i) il faudra peut-être consacrer des efforts particuliers pour sensibiliser ces entreprises à la nécessité de tenir compte des questions de sécurité chimique; ii) elles auront peut-être besoin d'aide additionnelle pour la mise en oeuvre des mesures proposées par les Principes directeurs.

Une approche polyvalente, comptant une grande variété d'entités et de programmes, pourrait être nécessaire pour faire face aux diverses préoccupations et limitations des PME (voir, par exemple, les paragraphes 1.19 et 2i.5-6).

" Les PME devraient commencer à :

- reconnaître l'importance de la sécurité comme partie intégrante de leurs activités et s'engager à faire fonctionner leur entreprise de façon sûre;

- rechercher activement l'information concernant la sécurité;

- former des partenariats avec les pouvoirs publics et (ou) d'autres entreprises afin d'améliorer la sécurité;

— créer des groupes $\mathrm{d}^{\prime}$ « aide mutuelle » d'intervention avec d'autres entreprises;

— joindre les rangs d'organisations professionnelles.

" Les autres intervenants devraient faire leur possible pour aider, si nécessaire, les PME à réduire les risques d'accident, en leur fournissant de l'information, des conseils et de l'assistance. Parmi ces intervenants, on peut citer :

- d'autres entreprises, incluant les fournisseurs et les groupes homologues;

- les pouvoirs publics, incluant les autorités locales;

- les associations commerciales ou industrielles, y compris les associations d'entreprises qui utilisent (sans les produire) des substances dangereuses;

- des groupes d'intervenants polyvalents, des organismes d'entraide, des partenariats industriegouvernement, des commissions de sécurité, des organisations professionnelles, des syndicats, des consultants, des établissements d'enseignement, des organisations non gouvernementales et des médias.

Les pouvoirs publics ainsi que les associations industrielles et commerciales devraient, si nécessaire, fournir des notes d'orientation sur la façon dont les PME peuvent se conformer à la réglementation.

L'OCDE a préparé une courte brochure, s'adressant directement aux directeurs de PME, afin de présenter les concepts figurant dans les Principes directeurs. Des copies de cette brochure peuvent être obtenues en communiquant avec l'OCDE, ou en se rendant sur le site Internet à l'adresse www.oecd.org/ehs/ (cliquer sur "Accidents chimiques", puis sur « En savoir plus», ensuite dans la boîte portant l'indication "Voir aussi », cliquer sur «Small and Medium-sized Enterprises (SMEs)». Le site présente d'autres renseignements utiles sur les PME. 


\section{Chapitre 2}

\section{INDUSTRIE (incluant la direction et les travailleurs)}

Dans le présent chapitre (traitant du rôle de l'industrie), on reconnaît que les entreprises sont les premières responsables de la prévention des accidents et que la sécurité chimique devrait former une partie intégrante de toutes les phases des activités d'une entreprise, allant de la conception et de la construction, jusqu'à la mise hors service/fermeture/démolition, en passant par l'exploitation et la maintenance. En plus d'examiner le rôle de la direction, le chapitre renferme également des clauses liées au rôle des travailleurs (catégorie définie comme étant constituée de tous les employés [y compris les contractants] autres que ceux de la direction, travaillant à ou pour une installation dangereuse).

\section{a. CULTURE DE LA SÉCURITÉ}

\section{Principes généraux}

2.a.1 Chaque entreprise devrait mettre en place et promouvoir une culture de la sécurité de l'entreprise, se traduisant par une politique de sécurité de l'entreprise.

" Une culture efficace de la sécurité est un élément essentiel de la gestion de la sécurité.

" La culture de la sécurité devrait découler des valeurs, des attitudes et des comportements de la haute direction et de leur circulation au sein de l'organisation. La culture de la sécurité commence avec l'engagement visible des membres du Conseil d'administration et des directeurs-exécutifs de l'entreprise, qui devraient montrer l'exemple et faire preuve d'initiative en s'engageant activement au niveau des questions de sécurité.

" En plus de cet engagement du haut vers le bas en matière de sécurité comme priorité, il devrait également y avoir un engagement du bas vers le haut, grâce à l'application active des politiques de sécurité par tous les employés. Allant de pair avec la culture de la sécurité, tous les employés devraient s'attacher à effectuer leur travail de façon sûre, en suivant les procédures établies et en aidant leurs collègues à relever ces défis.

" La culture de la sécurité devrait incorporer, comme élément fondamental, la conviction que tous les accidents peuvent être évités.

" Dans le cadre de sa culture de la sécurité, l'entreprise devrait établir des règles complètes concernant les rôles, les droits et les obligations de tous ceux qui sont concernés par l'assurance et le maintien de la sécurité.

" Pour la prévention efficace des accidents, des considérations concernant la sécurité devraient être incorporées, entre autres, dans les activités suivantes: planification, conception, construction et mise en service des installations; politiques et procédures d'exploitation, y compris les dispositions concernant l'organisation et le personnel; maintenance; fermetures temporaires; contrôle et surveillance de la sécurité; et enfin, mise hors service, fermeture et démolition des installations dangereuses. 
2.a.2 Dans le cadre de la culture de la sécurité, il devrait y avoir un engagement clairement établi et visible en faveur de la sécurité dans l'entreprise, visant à ce que tous les employés agissent de façon appropriée eu égard à la sécurité. Cet engagement se manifeste comme suit :

- intérêt clair et visible de la direction par l'engagement personnel de ses membres dans les questions de sécurité;

- efficacité de la communication sur les questions de sécurité au sein de la direction et entre celle-ci et d'autres employés;

- réaction positive aux mesures prises pour augmenter la sécurité;

- réponse rapide pour remédier aux erreurs décelées;

- incitatifs financiers et avancement pour une bonne performance en matière de sécurité;

- participation des employés à tous les niveaux à l'élaboration et à la modification des procédures de gestion de la sécurité;

- enquêtes en temps utile sur tous les accidents et quasi-accidents, et diffusion rapide des résultats de l'enquête.

2.a.3 La culture de la sécurité devrait encourager l'initiative et la vigilance dans l'intérêt de la sécurité.

" La culture de la sécurité devrait prévenir la complaisance et les lacunes au niveau de la structure et des procédures, qui toutes entrainnent des activités ou des pratiques contrevenant à la sécurité.

" Une caractéristique importante d'une culture efficace de la sécurité est la «tolérance de l'erreur »; une culture de la sécurité de ce type devrait permettre aux employés d'effectuer efficacement leurs tâches et de ne pas être obnubilés par l'évaluation d'un blâme ou par l'imposition de sanctions suite à des erreurs. La culture de la sécurité devrait encourager une atmosphère de coopération et d'ouverture, dans laquelle les employés se sentent à l'aise pour discuter d'erreurs et de quasi-accidents et ainsi mieux comprendre leur situation. Une culture tolérant l'erreur requiert néanmoins une responsabilisation appropriée et l'obligation de rendre des comptes.

" Pour promouvoir une culture de la sécurité de ce type, les employés et leurs représentants devraient avoir la possibilité de participer à l'élaboration et à l'examen de procédures et pouvoir prendre, sans crainte de représailles, des mesures appropriées en faveur d'une exploitation sûre et (ou) de la protection de la vie.

2.a.4 La direction devrait prendre les mesures appropriées pour s'assurer que tous les employés sont conscients de leurs rôles et responsabilités eu égard à la sécurité, et qu'ils possèdent les capacités, la formation, l'éducation, l'aide et les ressources voulues pour assumer ces rôles et responsabilités. La direction devrait veiller à ce que toutes les procédures de sécurité soient diffusées et bien connues et comprises par tous les employés (et d'autres personnes, si nécessaire).

(Voir plus loin la section 2.d «Exploitation", qui contient des clauses concernant le «Personnel » et la « Formation pratique et théorique »)

2.a.5 La direction et les autres employés ne devraient pas devenir trop confiants lorsqu'il n'y a pas eu d'accident à une installation pendant un certain temps; il ne faut pas cesser d'être vigilant si on veut maintenir la sécurité. 
2.a.6 La culture de la sécurité d'une entreprise peut être améliorée si la direction a une attitude ouverte vis-à-vis du public pour ce qui est des questions de sécurité.

\section{Politique de sécurité}

2.a.7 Chaque entreprise devrait élaborer une déclaration claire et sensée de sa politique de sécurité acceptée, promulguée et appliquée dans toute l'entreprise, déclaration qui reflète la culture de la sécurité de l'entreprise, contient les objectifs et principes généraux en matière de sécurité chimique, et incorpore l'objectif «zéro incident» ainsi que les objectifs de sécurité établis par les pouvoirs publics.

" La politique de sécurité doit se situer au sommet hiérarchique d'une documentation liée à la sécurité chimique d'une entreprise, chaque niveau successif expliquant de façon de plus en plus détaillée l'application de la politique et incluant les documents de travail et les instructions.

" La politique devrait couvrir les mesures de prévention, de préparation et d'intervention en matière d'accident, y compris les éléments du système de gestion de la sécurité (voir plus loin «Systèmes de gestion de la sécurité »).

" La politique de sécurité devrait viser à protéger la sécurité et la santé de toute personne engagée dans la production, le traitement, la manipulation, l'utilisation, l'entreposage ou l'élimination de substances dangereuses, ou pouvant être touchée par l'une de ces activités, et protéger l'environnement et les biens.

" La politique de sécurité devrait être revue régulièrement et, si nécessaire, modifiée, à la lumière de l'expérience acquise et de tout changement pertinent dans les technologies, les lois et les règlements.

2.a.8 Lors de l'élaboration, de la revue et de la modification de la Politique de sécurité, la direction devrait consulter les employés à tous les niveaux et faire appel à leur engagement. Les employés responsables du développement des politiques de sécurité de l'entreprise ne devraient pas relever de ceux qui sont chargés de la direction de la production, et ils devraient avoir accès directement à la haute direction.

2.a.9 La politique de sécurité devrait être diffusée largement dans toute l'entreprise. La direction devrait s'assurer que la raison d'être de cette politique est bien comprise et bien acceptée par tous les employés de l'entreprise.

2.a.10 La gestion et d'autres employés devraient coopérer de façon à se conformer à la politique de sécurité de l'entreprise et à atteindre ses objectifs en matière de sécurité.

" Aux fins de la prévention des accidents, la direction et les travailleurs ont des rôles et des responsabilités différents, mais complémentaires lorsqu'ils effectuent leurs tâches de façon sûre, en contribuant activement à l'élaboration et à la mise en oeuvre des politiques et des pratiques en matière de sécurité, et en coopérant les uns avec les autres et avec d'autres intervenants.

" Les employés à tous les niveaux devraient être motivés et formés (pratique et théorie) pour faire de la sécurité une haute priorité, et de son amélioration permanente un objectif important pour l'entreprise. 
4 Les travailleurs et leurs représentants devraient coopérer avec la direction pour promouvoir la sécurité chimique, et ils devraient disposer de moyens efficaces (structures et procédures) pour ce faire.

2.a.11 La politique de sécurité devrait être rendue accessible au public.

2.a.12 Chaque installation faisant partie d'une entreprise devrait élaborer et mettre à jour son propre programme de sécurité, conforme à la politique de sécurité de l'entreprise et traitant de façon plus détaillée des questions de sécurité et des exigences spécifiques à cette installation. Ce programme doit être élaboré avec la participation active des employés à tous les niveaux et faire régulièrement l'objet d'une révision.

" À chaque installation, la responsabilité de la gestion quotidienne de la sécurité devrait relever du cadre hiérarchique.

" Le cadre hiérarchique devrait répondre aux propositions et aux suggestions des travailleurs et de leurs représentants concernant les questions de sécurité, ou les transmettre à son superviseur.

" La haute direction devrait fournir aux cadres hiérarchiques le support nécessaire pour les décisions et les mesures concernant la sécurité.

2.a.13 L'élaboration et la mise en oeuvre d'une politique de sécurité par une entreprise ainsi que les pratiques liées à la prévention et à la préparation pour les accidents devraient être coordonnées et intégrées aux activités de l'entreprise concernant d'autres aspects de la sécurité professionnelle, de la santé et de la protection de l'environnement, dans le cadre d'un programme de gestion du risque total.

" On devrait s'orienter vers la gestion intégrée de la sécurité, de la santé et de l'environnement (SSE) dans toutes les activités régulières d'exploitation d'une entreprise. Cela permettrait généralement de contribuer au développement durable. À cet égard, il faudrait être conscient du fait qu'il n'est pas possible de réaliser le développement durable sans placer la barre très haute en matière de sécurité et de protection de la santé et de l'environnement.

" La gestion de la sécurité devrait représenter une partie intégrante de la gestion totale de la qualité (GTQ).

4 L'intégration des systèmes de gestion pour les questions d'environnement, de santé et de sécurité, et l'élaboration de procédures à l'échelle de l'entreprise, applicables à toutes les installations, permettent d'améliorer la sécurité. L'utilisation de ces procédures peut aider à détecter les situations qui pourraient entraîner des accidents de travail ainsi que des carences organisationnelles ou d'autres erreurs pouvant à leur tour causer des rejets de substances dangereuses.

\section{Systèmes de gestion de la sécurité}

2.a.14 La direction devrait mettre en place un système de gestion de la sécurité (en tant que partie totalement intégrée de son système général de gestion), qui s'occupe de la prévention, de la préparation et de l'intervention en matière d'accidents chimiques. Le 
système de gestion de la sécurité devrait comprendre la structure organisationnelle, les pratiques, les procédures et les ressources pour la mise en oeuvre de la politique de sécurité.

" Un système efficace de gestion de la sécurité constitue une bonne pratique commerciale. Les faits montrent qu'un tel système apporte des avantages financiers directs et indirects, par exemple en réduisant les coûts associés aux accidents ou aux mises hors service avec perte de temps, en améliorant le capital de sympathie, enfin, en diminuant les primes d'assurance.

" Le système de gestion de la sécurité devrait refléter la culture de la sécurité de l'entreprise, laquelle devrait s'engager du haut vers le bas, et ce à partir de son plus haut niveau, en faveur du système.

" Il devrait y avoir un engagement du bas vers le haut, de la part de tous les employés en faveur du système de gestion de la sécurité. L'entreprise devrait appeler les employés et leurs représentants à participer à l'élaboration du système de gestion de la sécurité, de telle façon qu'ils se sentent partie prenante du système et qu'ils puissent lui faire confiance.

" Des ressources et un personnel suffisants devraient être prévus pour la mise en oeuvre du système de gestion de la sécurité. Il devrait y avoir une attribution clairement définie des responsabilités pour chaque élément.

2.a.15 Le système de gestion de la sécurité devrait s'appliquer au moins aux domaines suivants :

" Structure organisationnelle (incluant les rôles, les responsabilités, la formation pratique et théorique, les qualifications et les relations interpersonnelles dans les tâches dévolues à la sécurité).

" Identification et évaluation des dangers (élaboration et mise en oeuvre de procédures bien définies pour identifier et évaluer les dangers- notamment leur probabilité et leur gravité - résultant d'activités normales ou anormales, et comprenant les dangers générés par la manipulation, la production, le transport, l'entreposage ou l'élimination de substances).

" Contrôle des installations et maîtrise opérationnelle (pour la conception et la construction, ainsi que les procédures pour une exploitation sûre, incluant la maintenance de l'installation, les procédés, l'équipement et les arrêts temporaires).

" Gestion du changement (planification et contrôle des changements dans les éléments suivants : organisation; personnel; installation; procédés, notamment les études préalables à la mise en route, maintenance et mise hors service; produits; équipement; procédures; logiciels; conception; enfin, toute condition externe pouvant influer sur la sécurité).

" Planification pour les situations d'urgence (relative à l'élaboration, à l'adoption, à l'application, à l'examen, à l'essai et, au besoin, à la révision et à la mise à jour des plans d'urgence). 
" Contrôle de la performance (concernant l'évaluation régulière de la conformité à la politique de sécurité et au système de gestion de la sécurité, ainsi que les mécanismes pour les mesures correctives à prendre en cas de non-conformité).

" Audit et réexamen (pour l'évaluation régulière et systématique de la politique de sécurité, ainsi que de l'efficacité et de la pertinence du système de gestion de la sécurité).

" Enquête sur les accidents et apprentissage par l'expérience.

\section{Rapports sur la sécurité}

2.a.16 La direction d'installations dangereuses devrait produire des rapports décrivant les dangers chimiques les plus importants à ces installations et démontrant que les étapes appropriées ont été suivies pour empêcher les accidents chimiques et limiter leurs effets ${ }^{4}$.

2.a.17 Ces rapports devraient démontrer que les étapes appropriées sont suivies pour gérer les dangers chimiques. Les rapports devraient être réexaminés régulièrement et, si nécessaire, mis à jour. Ils devraient inclure une description ou une référence à des documents portant sur les points suivants :

" L'installation, avec son utilité, ses activités, son diagramme de fonctionnement, ses dangers intrinsèques, ses substances dangereuses, son personnel, ses services et son équipement technique.

4 La zone entourant l'installation, incluant les milieux sensibles, la population et les activités à l'intérieur de cette zone (notamment les activités commerciales, résidentielles et industrielles).

" L'identification des dangers et l'évaluation des risques représentés par une installation (voir section $2 b$ sur l'identification des dangers et l'évaluation des risques).

" Le plan d'urgence interne, incluant la liaison avec les plans externes ainsi que la communication et la coordination avec le personnel des interventions d'urgence (voir chapitre 5, section b sur la préparation et la planification pour les situations d'urgence industrie).

" La politique de sécurité de l'entreprise (voir paragraphe 2.a.7-13).

" Le système de gestion de la sécurité de l'entreprise (voir paragraphe 2.a.14-15).

" Les procédures pour la déclaration interne des incidents (voir chapitre 14, section c traitant de la constitution de dossiers sur les installations et des déclarations industrie)

2.a.18 Ces rapports devraient être présentés aux pouvoirs publics pour examen. 


\section{b. IDENTIFICATION DES DANGERS ET ÉVALUATION DES RISQUES}

Dans ce contexte, l'évaluation des risques est un outil employé dans la gestion des risques pour mieux comprendre les risques et documenter la sélection et la priorisation des stratégies de prévention et de contrôle. Grâce à l'évaluation des risques, ces derniers peuvent être classés selon une échelle relative, et on peut alors évaluer les options techniques/organisationnelles/politiques, ce qui permet de maximiser les résultats pour une sécurité optimale. Cela facilite le choix d'options. L'évaluation des risques permet également de fournir de l'information à ceux qui élaborent les politiques pour les aider à déterminer les critères d'acceptabilité ou de tolérabilité du risque, en fonction desquels on peut évaluer différents objectifs ou programmes.

L'évaluation des risques est un processus qui comprend un certain nombre d'étapes, à savoir: l'identification des dangers; l'évaluation du scénario d'un événement; l'évaluation des conséquences; l'évaluation de la probabilité; l'intégration et la comparaison du risque ${ }^{5}$.

2.b.1 La direction devrait entreprendre l'identification des dangers et l'évaluation des risques pour toutes les installations dangereuses.

2.b.2 Lorsqu'elle entreprend une évaluation des risques, la direction doit bien tenir compte des diverses approches possibles et des méthodes disponibles. Elle devrait choisir une approche/méthode qui soit appropriée aux conditions particulières, en effet toutes les approches/méthodes ont des forces et des faiblesses, et aucune n'est parfaite.

" Le choix d'une approche/méthode particulière devrait être régi par un certain nombre de facteurs, dont les suivants :

- objectif/raison d'être de l'évaluation du risque;

- estimation de la nature du risque;

- disponibilité et suffisance des données;

- expertise et ressources nécessaires et disponibles pour une approche/méthode particulière;

- historique des incidents à l'installation et à d'autres installations apparentées;

- contraintes inévitables s'exerçant sur le processus;

- contexte socio-politique dans lequel est effectuée l'évaluation; et,

- hypothèses sur lesquelles est fondée l'approche/méthode.

" Pour choisir une approche/méthode appropriée pour l'évaluation du risque, il faudrait d'abord clairement définir les objectifs de cette évaluation.

2.b.3 Les évaluations du risque devraient être accompagnées de l'information concernant les hypothèses, les limitations dans les données et les incertitudes incorporées dans les approches/méthodes d'évaluation du risque et dans les processus décisionnels, de telle façon que les résultats de ces évaluations puissent être utilisés de façon appropriée.

" Il est important de tenir compte des possibles limitations de données et du choix inapproprié de données si on veut que les résultats des évaluations soient fiables et exhaustifs.

" Par exemple, il peut y avoir des disparités et des insuffisances dans les données disponibles, par exemple, sur les taux et modes de défaillance de l'équipement, les prévisions d'erreurs humaines, les effets à long terme ou retardés d'une exposition aiguë sur la santé, ou encore sur les effets de substances chimiques sur l'environnement. 
" Les limitations au niveau des données peuvent être gérées en partie grâce à l'emploi d'approches/méthodes moins détaillées et plus générales, ou à l'aide d'évaluations comparatives pour faciliter le choix parmi plusieurs options. Les évaluations comparatives font généralement appel à des hypothèses, limitations et incertitudes similaires avec, comme conséquence, l'annulation de leurs effets sur les résultats de l'évaluation.

2.b.4 Toutes les parties doivent rechercher une plus grande clarté dans les hypothèses à la base de l'évaluation des dangers et des risques, ainsi qu'une plus grande transparence dans le processus d'évaluation, de façon à permettre une communication plus efficace et une meilleure compréhension, et enfin à faciliter les comparaisons.

" Pour les hypothèses qui ne peuvent être éliminées, il est souhaitable d'obtenir le consensus entre toutes les parties engagées dans le processus décisionnel, à défaut de quoi l'évaluation pourrait manquer de crédibilité et de support.

" Toute initiative pour améliorer la cohérence et la communication concernant les risques devrait tenir compte des diverses méthodes employées par différents pays et organisations.

2.b.5 Les intervenants touchés, y compris les représentants du public, devraient avoir un rôle à jouer dans le processus d'évaluation du risque, particulièrement en ce qui concerne l'évaluation du niveau de signification des résultats. Les décisions qui dépendent des évaluations du risque peuvent avoir une importance fondamentale pour, par exemple, les employés, la population pouvant être touchée en cas d'accident et le personnel d'intervention en cas d'urgence.

" L'engagement des intervenants est important si on veut prendre les décisions appropriées, instaurer la confiance dans les résultats et faciliter un dialogue ouvert et constructif.

" Le processus d'évaluation du risque devrait être transparent, et tous les intervenants devraient être conscients de la capacité et des limites du processus d'évaluation du risque et des approches ou méthodes adoptées.

"Le fait que les personnes qui prennent les décisions concernant la gestion du risque ne sont pas nécessairement les mêmes que celles qui évaluent les risques montre que le processus d'évaluation du risque doit être transparent et qu'il faut une vision commune des concepts qui sont à la base de la terminologie de l'évaluation du risque.

2.b.6 Les scénarios d'accidents considérés comme faisant partie du processus d'évaluation du risque devraient tenir compte de la possibilité d'erreurs humaines et technologiques ainsi que de catastrophes naturelles et (ou) d'actes délibérés (p.ex. sabotage, terrorisme, vandalisme ou vol), comme éléments déclencheurs d'un accident chimique.

2.b.7 Les évaluations du risque dans le contexte d'installations dangereuses devraient tenir compte de toutes les conséquences possibles, y compris de celles pour l'environnement et la santé. 
" Lorsque la mort ou les effets sur la santé sont les seuls paramètres utilisés dans le processus d'évaluation du risque, le processus peut conduire à des conclusions ambiguës ou erronées.

" La direction et les pouvoirs publics devraient être proactifs en recherchant des moyens pour améliorer l'évaluation du risque eu égard aux conséquences des accidents chimiques pour l'environnement.

" L'évaluation des risques pour l'environnement est complexe, mais ce n'est pas une raison pour l'éviter; il faut plutôt s'attaquer au problème en commençant par de simples modèles qui peuvent être raffinés avec le temps.

"L'évaluation des conséquences environnementales des accidents est complexe principalement à cause de l'insuffisance des données et des outils permettant de réaliser ces évaluations et de déterminer des moyens pour prévenir et atténuer ces conséquences. Les enjeux sont les suivants :

- le grand nombre de récepteurs potentiels dans l'environnement (p. ex. flore, faune), la connaissance incomplète de la façon dont chacun de ces récepteurs est altéré par l'exposition aux substances chimiques;

- le grand nombre de voies possibles (p. ex. sol, eaux souterraines, eaux de surface, air);

- la connaissance limitée de la façon dont les substances chimiques peuvent se déplacer, se disperser, réagir et se transformer dans l'environnement;

- l'insuffisance des données sur les aspects environnementaux des accidents antérieurs.

" Souvent, des substances que l'on sait inoffensives pour l'environnement peuvent, en combinaison avec d'autres produits et (ou) d'autres facteurs, engendrer des dangers significatifs, ou encore il peut y avoir un effet synergiste qui fait que de petites quantités de substances chimiques peuvent produire des impacts importants.

" On devrait s'employer à partager entre les divers pays l'expérience sur la mise au point et l'application de méthodes et d'outils servant à l'évaluation des risques pour l'environnement.

2.b.8 L'évaluation du risque devrait être un processus continu et évolutif. Les évaluations doivent être réexaminées et reprises à intervalles réguliers ou lorsqu'on décèle des signes montrant qu'une révision s'impose.

" Par exemple, il faudrait revoir l'évaluation du risque :

- lorsque de nouveaux procédés ou des procédés modifiés sont utilisés à des installations dangereuses, ou lorsque des modifications importantes ont été apportées au transport des substances dangereuses;

- suite à des incidents;

- si une nouvelle technologie laisse entrevoir des possibilités d'amélioration;

- s'il y a une certaine contradiction entre l'expérience des travailleurs et (ou) de la direction et l'évaluation du risque;

- si de nouvelles données apparaissent au sujet du comportement ou des effets des substances et des procédés;

- s'il y a des projets de nouvelles constructions ou d'autres développements à l'intérieur du site de l'installation ou à proximité. 
" De plus, les évaluations du risque devraient être revues régulièrement pour tester les hypothèses, tenter de résoudre les incertitudes, tirer avantage de l'expérience et des améliorations apportées aux méthodes.

2.b.9 Les entreprises et les organisations industrielles devraient échanger l'information concernant les méthodes d'évaluation du risque et leurs résultats obtenus de façon à améliorer la compétence au niveau de l'emploi des stratégies/méthodes d'évaluation du risque. Cet échange d'information peut également être utilisé pour faciliter la formation destinée à augmenter l'expertise disponible ${ }^{6}$.

\section{c. IMPLANTATION, CONCEPTION ET CONSTRUCTION}

\section{Implantation des installations}

\section{(Voir section $3 . b$ et chapitre 6 traitant de l'aménagement du territoire)}

2.c.1 Lorsqu'elle choisit des sites possibles pour de nouvelles installations dangereuses, la direction d'une entreprise doit respecter l'aménagement du territoire ainsi que les exigences et lignes directrices en matière de zonage. La direction doit rechercher des sites qui réduiraient au minimum les effets nuisibles pour la santé, l'environnement et les biens en cas d'accident à l'installation ou lors du transport de substances dangereuses vers l'installation ou en provenance de celle-ci. La direction et les pouvoirs publics (notamment ceux qui prennent les décisions en matière d'aménagement du territoire) devraient coopérer pour faire en sorte que les installations dangereuses soient implantées et construites de façon à réduire au minimum les risques pour la santé humaine, l'environnement et les biens.

2.c.2 La direction d'une entreprise qui projette de construire une nouvelle installation dangereuse ou d'apporter des modifications importantes à une installation existante devrait élaborer un plan à l'échelle du nouveau projet. Ce plan devrait refléter les données communiquées par les pouvoirs publics et montrer les éléments suivants :

- emplacement et quantités de substances dangereuses présentes sur le site par rapport à la zone environnante;

- type d'aménagement du territoire dans les zones voisines;

- population locale et zones présentant localement un intérêt environnemental;

- effets potentiels générés par le projet à l'extérieur de l'installation.

" La direction devrait également décrire les diverses parties des procédés où il y a présence de substances dangereuses, l'inventaire de substances dangereuses à entreposer, et les conditions dans lesquelles les substances dangereuses doivent être manipulées. De plus, la direction devrait évaluer les conséquences de l'installation projetée pour la santé humaine et l'environnement.

" Ces activités liées à l'évaluation devraient être conduites en liaison avec les autorités locales et le public le plus tôt possible au début du processus de planification pour l'installation, de façon à faciliter les décisions d'implantation et l'étude de solutions de remplacement rentables.

" Le plan à l'échelle, avec l'information et l'évaluation connexes, devraient être fournies aux autorités compétentes. 
2.c.3 La direction des installations dangereuses et les pouvoirs publics devraient examiner ensemble les moyens permettant de réduire les risques aux installations existantes, de telle façon qu'elles soient conformes aux lois et lignes directrices actuelles en matière d'aménagement du territoire et de zonage (p.ex. si les lois ou lignes directrices ont changé depuis que l'installation a été construite, ou si des habitations ou d'autres constructions ont été aménagées au voisinage de l'installation).

" La direction devrait, si nécessaire, faire preuve de bonne volonté pour réduire les risques aux installations existantes, de façon à les rendre conformes aux lois et lignes directrices en vigueur.

" La direction devrait travailler avec les autres intervenants de la communauté pour empêcher la construction d'habitations et d'autres développements (y compris, par exemple, écoles, hôpitaux et centres commerciaux) à proximité de leurs installations dangereuses, si cela peut entraîner des risques inacceptables pour la santé, l'environnement ou les biens à l'extérieur de l'installation.

\section{Conception, planification et aménagement}

- 2.c.4 Des mesures de sécurité devraient être incorporées aux premiers stades de l'étude de définition et de la conception technique d'une installation, de façon à améliorer la sécurité intrinsèque (inhérente) de celle-ci, là où c'est applicable.

" L'utilisation d'une technologie intrinsèquement plus sûre ${ }^{7}$ pour la production, le transport et l'utilisation de substances chimiques (p. ex. réduction des inventaires de substances dangereuses, utilisation de procédés de production plus sûrs et amélioration des enceintes secondaires) permet d'améliorer la sécurité des installations dangereuses.

" Il faudrait concevoir des procédés pour contenir, contrôler et réduire au minimum la quantité de substances dangereuses intermédiaires, de façon à améliorer la sécurité. Lorsque cela n'est pas possible, la quantité de substances intermédiaires produite devrait être réduite au niveau requis pour la prochaine étape de production, ce qui permet de maintenir à un strict minimum les quantités entreposées.

" Les mesures de sécurité devraient tenir compte des possibilités d'erreurs humaines et (ou) techniques, ainsi que d'actes délibérés, comme le sabotage ou le vandalisme, pouvant se produire à une installation.

" Les mesures de sécurité devraient permettre de se conformer sans trop de difficultés aux procédures de sécurité.

" La conception des installations dangereuses devrait tenir compte du facteur humain et des limitations humaines, et s'aligner sur les principes d'ergonomie. Elle devrait également considérer les capacités et limitations psychologiques, physiologiques et cognitives des personnes qui assument des tâches importantes aux installations dangereuses. À cet égard, la conception doit simplifier le plus possible les interventions des opérateurs en tenant compte des capacités cognitives de ces derniers, ce qui permettra de réduire au minimum la possibilité d'erreurs. 
" Pour éviter de concevoir une installation comportant des erreurs latentes de fonctionnement, des essais devraient être effectués pour déterminer si la conception de fonctionnement de l'installation est faisable et applicable (p.ex. qu'elle tient compte de la quantité limitée d'information qui peut être traitée par des personnes dans les conditions auxquelles des opérateurs peuvent être confrontés dans une installation).

" Les principes de conception technique concernant la sécurité ne s'appliquent pas seulement aux nouvelles usines et aux nouveaux procédés, mais également aux modifications d'installations et de procédés existants, ainsi qu'aux activités de recherche.

2.c.5 Pour atteindre un haut niveau de sécurité, la conception des nouvelles installations ou les modifications importantes apportées à des installations existantes devraient incorporer les normes internationales, les codes de pratique et les lignes directrices les plus pertinents et les plus récents, tels qu'établis par les pouvoirs publics, les entreprises, l'industrie, les associations professionnelles et d'autres organismes.

4 Cependant, ces normes, codes de pratique et lignes directrices devraient être considérés comme des exigences minimales. L'amélioration de la sécurité est un processus dynamique, qui devrait refléter les progrès dans les connaissances et la technologie. Ces normes, codes et lignes directrices devraient donc être complétés par d'autres lignes directrices élaborées dans le cadre de l'entreprise (incorporées dans des guides et des spécifications pour la conception technique) et résultant de l'expérience opérationnelle et des connaissances spécialisées acquises.

" Les installations existantes devraient être évaluées afin de déterminer si elles respectent ces normes, codes et lignes directrices. Lorsqu'elles ne les respectent pas, les améliorations appropriées devraient être apportées le plus tôt possible.

2.c.6 La conception d'une installation dangereuse devrait intégrer l'équipement, le matériel et les procédures techniques qui permettraient de réduire le plus possible les risques (autrement dit, toutes les mesures devraient être prises pour réduire les risques avant de considérer des dépenses additionnelles pour aller bien au-delà de l'amélioration de la sécurité résultante).

" À cet égard, pour réduire le risque, il faudrait envisager d'utiliser un procédé et une conception «intrinsèquement plus sûrs » pour l'installation. Des approches intrinsèquement plus sûres consisteraient à choisir soigneusement le procédé, ainsi qu'une conception judicieuse pour l'installation (en fait, à opter pour une conception éliminant certains risques, à réduire au minimum les conséquences des erreurs humaines, et à mieux tolérer les erreurs pouvant survenir). Ce type d'approche comprend les concepts suivants, dans la mesure où ils réduisent le risque total :

- réduire les inventaires de substances dangereuses;

- réduire au minimum, dans la mesure du possible, l'emploi de substances dangereuses;

- remplacer les substances dangereuses par d'autres moins dangereuses;

- utiliser des substances ou des procédés dangereux de façon à limiter le danger potentiel qu'ils représentent (p.ex. grâce à des systèmes en circuit fermé, utilisant des substances moins réactives);

- faciliter la conception, l'aménagement et l'exploitation des usines et des procédés; 
- modérer les conditions de fonctionnement (p. ex. les niveaux de pression);

- passer des systèmes complexes à des systèmes plus simples;

- adapter la nature et le développement du transport à l'intérieur de l'installation et hors de celle-ci;

- s'assurer qu'il y a des systèmes de sécurité en double;

- réduire au minimum la production de déchets dangereux.

" Des systèmes conçus spécialement pour augmenter la sécurité du procédé - avec, par exemple, système de décompression et détecteur d'incendie et d'explosion devraient être inclus dans la conception technique des installations dangereuses nouvelles et existantes, en tenant compte des scénarios d'accidents possibles.

" Les installations dangereuses devraient être conçues de façon à prévenir ou à réduire au minimum l'exposition des employés aux substances dangereuses, ce qui permet de réduire les besoins en équipement de protection individuelle.

" Dans le cas de l'équipement essentiel pour la sécurité (comme les contenants sous pression ou les instruments de contrôle), la conception technique devrait être soumise à une certification reconnue ou à une procédure de vérification.

" Pour la conception des installations dangereuses, on devrait considérer l'emploi de systèmes en double aux fins de la sécurité (comme l'alimentation en électricité pour les systèmes de contrôle).

" Il faudrait aussi envisager de protéger au maximum les parties vulnérables de l'entreprise contre les dommages causés par des actions externes (p. ex. sabotage, terrorisme, vandalisme, vol).

2.c.7 Les principes d'une conception intrinsèquement plus sûre ne devraient pas être appliqués isolément, mais plutôt faire partie d'une démarche intégrée pour améliorer la sécurité.

" Cela concerne l'exploitation et la maintenance de l'installation ainsi que l'application de systèmes de gestion de la sécurité, et on devrait considérer notamment les éléments suivants : l'intégrité continue de l'équipement dans le temps, la gestion du personnel, la gestion du changement, la formation des opérateurs et d'autres employés, les réexamens et les audits de la performance en matière de sécurité, l'apprentissage par l'expérience et la mise en place d'une culture de la sécurité.

" Une bonne conception devrait être complétée par des systèmes de gestion appropriés de la sécurité, incluant les éléments suivants : formation, recyclage et éducation des employés; élaboration, mise en oeuvre, réexamen et mise à jour des procédures d'exploitation; gestion prudente des modifications dans la conception; conséquences pour la sécurité lorsqu'il y a des changements au niveau de la direction ou d'autres employés; procédures d'audit et de contrôle.

2.c.8 Même si, au niveau de la conception et de l'exploitation, on devrait mettre l'accent sur la sécurité intrinsèque, il faudrait également considérer la nécessité de systèmes protecteurs "additionnels", ce qui permettrait de garantir la sécurité grâce à des mesures d'atténuation. 
" Les procédures devraient être conçues de façon à réduire au minimum le risque de panne et, en cas de panne, de façon à réduire au minimum les effets nuisibles.

" Les systèmes ou mécanismes (utilisant, par exemple, des parois de confinement ou des bassins collecteurs) permettant de contenir les fuites, les déversements accidentels ou l'eau pour combattre un incendie devraient eux aussi être incorporés dans la conception des installations dangereuses, en tenant compte de la quantité de substances dangereuses qui pourraient être libérées. Ces systèmes ou mécanismes pourraient également comporter un plus grand nombre de barrières pour prévenir le rejet de ces substances, p. ex. une double encapsulation.

4. S'il y a un bris dans l'enceinte de confinement, on peut réduire au minimum les effets nuisibles par d'autres mesures d'atténuation, comme l'emploi d'équipement de protection contre l'incendie et les procédures en cas d'urgence.

2.c.9 Les systèmes devraient être conçus de telle façon qu'une panne dans une composante individuelle n'engendre pas des conditions non sécuritaires pour le procédé (autrement dit, ils doivent être à $l^{\prime}$ «épreuve des pannes») et (ou) devraient pouvoir tolérer de possibles erreurs humaines. Lorsque les employés sont bien formés, ils peuvent réagir de façon appropriée dans des situations anormales ou imprévues, notamment lorsqu'ils disposent d'un peu de temps pour réagir. La conception des installations dangereuses devrait donc tenir compte de cela et prévoir un peu de temps pour permettre à l'opérateur de réagir avant qu'il n'y ait d'autres dommages en cas de situation anormale.

2.c.10 Un niveau approprié d'automatisation et des systèmes d'aide à la décision devraient être incorporés dans la conception des installations dangereuses.

4 L'automatisation complète n'est ni réaliste ni optimale du point de vue de la sécurité. Même si l'automatisation et les systèmes d'aide à la décision peuvent améliorer la sécurité grâce à la rapidité du diagnostic et de la réaction, ces systèmes ne répondent qu'à des événements anormaux prévus ou «connus». Les événements qui ne se situent pas dans le cadre des spécifications de conception, ou qui ne sont pas prévus, doivent être traités manuellement. La présence d'un opérateur bien renseigné et bien formé pour réagir est donc indispensable.

" Si le système est automatisé dans des proportions telles que l'opérateur n'a plus que très peu de responsabilités, ce dernier n'aura peut-être plus la capacité ou l'expérience suffisante pour faire face à des situations anormales se produisant rarement. La sécurité peut également être compromise si les responsabilités de l'opérateur deviennent trop banales, ou si l'opérateur n'a pas assez d'occasions pour mettre en pratique ses compétences.

" Même s'il y a l'automatisation, il faut néanmoins maintenir un niveau suffisant de personnel pour avoir une présence régulière sur place dans l'unité opérationnelle (et pas seulement à distance, à des ordinateurs) et ainsi disposer d'un personnel suffisant pour faire face aux situations d'urgence.

\section{(Voir paragraphes 2.d.10 - 17 concernant le personnel)}

2.c.11 Les systèmes informatisés peuvent être une composante importante de la sécurité d'exploitation. 
" Les systèmes en ligne devraient aider les opérateurs à assumer leurs responsabilités et permettre un accès facile et rapide aux procédures d'exploitation et à l'information connexe.

" Les systèmes en ligne devraient également permettre de recueillir l'information utile pour déterminer les causes fondamentales des incidents, alors que les systèmes hors ligne devraient donner un accès facile et rapide à la documentation sur l'entreprise, à la planification d'urgence ainsi qu'à la formation et à l'enseignement.

2.c.12 Les systèmes de sécurité, qu'ils soient informatisés ou qu'ils requièrent une intervention humaine, devraient être conçus et testés de telle façon que les signaux critiques parviennent jusqu'à l'opérateur (même s'il y a plusieurs pannes simultanées) et que les systèmes ne soient pas surchargés et, par conséquent, inopérants.

" Par exemple, on devrait considérer le fait qu'un opérateur peut être inondé de signaux si plusieurs systèmes d'une même installation font défaut en même temps, entraînant de la confusion par suite de la trop grande abondance d'information et de l'incapacité de déterminer la marche à suivre appropriée. À cet égard, il est plus difficile d'interpréter une information hautement intégrée que des données individuelles simples.

" Pour la conception des systèmes de sécurité, il est essentiel de tenir compte des effets psychologiques pouvant s'exercer sur un directeur ou un opérateur lorsque des systèmes tombent en panne, et de la façon dont ce type de stress peut altérer leur capacité à réagir.

2.c.13 Dans la phase de la conception, la direction devrait s'assurer que l'on tient suffisamment compte de l'aménagement du site, avec comme éclairage les objectifs généraux de sécurité. Il faudrait tout particulièrement considérer les éléments suivants :

" Établissement de distances de séparation sûres pour réduire au minimum tout effet ${ }^{8}$ de type "onde de choc» ou "effet domino" sur le site, dans les limites de l'installation, ou hors-site et mettant en jeu d'autres entreprises.

" Localisation des substances et procédés dangereux par rapport au lieu de travail du personnel ainsi qu'à l'équipement et aux contrôles de sécurité nécessaires.

" Localisation des bureaux, des salles de commande et d'autres locaux, de façon à réduire au minimum les effets nuisibles sur la santé et à augmenter la capacité de garder le contrôle sur l'installation en cas d'accident.

"Effets possibles sur la communauté et l'environnement locaux.

2.c.14 Une installation d'entreposage ou toute autre installation dangereuse qui entrepose des substances dangereuses devrait être conçue en fonction de la nature et de la quantité des substances dangereuses à entreposer.

" La conception des installations d'entreposage devrait incorporer des éléments de sécurité permettant de réduire au minimum la probabilité et la gravité d'un accident mettant en cause des substances dangereuses. À cette fin, la conception devrait prévoir la séparation des substances incompatibles et la subdivision des inventaires grâce à, par exemple, des bâtiments séparés ou des murs pare-feu. De plus, 
l'installation devrait être conçue de façon à réduire le risque d'effet domino en cas d'accident.

" Il faut tout particulièrement veiller à mettre en place des systèmes automatisés pour la manipulation des substances dangereuses, ce qui réduit le risque d'un accident mettant en cause ces substances.

" De plus, la conception devrait permettre d'accéder aux lieux pour les inspections concernant les substances dangereuses, de combattre les incendies et d'organiser efficacement l'évacuation. L'équipement pour combattre les incendies devrait être disponible et des systèmes de retenue adéquats (p.ex. aires entourées d'un mur) devraient être prévus pour faciliter l'activation des procédures d'atténuation en cas de déversement afin de protéger les personnes et l'environnement si un accident se produit. Des mesures de sécurité devraient également être en place, notamment une clôture et l'accès limité au personnel autorisé.

" On devrait s'employer à réduire au minimum la quantité de substances dangereuses à une installation d'entreposage, et ce dans des proportions permettant de réduire le niveau total de risque.

2.c.15 Des précautions devraient être prises pour faire en sorte que les choix ou les modifications apportées n'augmentent pas ou ne transfèrent pas involontairement le risque. Par exemple, dans certains cas, la réduction des inventaires de substances dangereuses peut augmenter le risque total en raison de la nécessité d'activités de transport et de manipulation plus fréquentes (p. ex. chargement et déchargement des substances).

2.c.16 Le personnel compétent qui sera responsable de l'exploitation d'une installation dangereuse devrait également être engagé dans les diverses phases de planification, de conception et de construction de l'installation. Les employés et leurs représentants devraient participer aux décisions concernant la conception de leur lieu de travail, et on devrait leur donner la possibilité de participer à la conception, à la mise en place et à l'amélioration de l'équipement, de façon à profiter du savoir-faire et de l'expérience des employés.

2.c.17 La direction d'une installation dangereuse devrait rassembler toute l'information liée à la sécurité concernant le procédé et l'équipement connexe, notamment les données relatives à la conception, à l'exploitation, à la maintenance et aux situations d'urgence.

" Un fichier ou un dossier de ce type est essentiel aussi bien pour la formation qu'à des fins d'exploitation.

" Ce fichier ou dossier est également nécessaire pour la production de rapports sur la sécurité, qui peuvent être requis par les pouvoirs publics, ou aux fins d'inspection ou de contrôle par ces derniers.

" Le concept ou les procédures d'exploitation devraient contenir des informations sur les caractéristiques relatives à la sécurité incorporées dans la conception (y compris les systèmes de sécurité automatisés), ainsi que sur le rôle des opérateurs, des directeurs, du personnel chargé de la maintenance, etc. De plus, le fichier de documentation sur le procédé ou le dossier sur l'installation devrait contenir de l'information sur les éléments suivants : 
- procédures de fabrication;

- instructions pour le procédé et l'exploitation (y compris la mise en route et l'arrêt en toute sécurité);

- diagrammes linéaires de fonctionnement du procédé, montrant les pièces d'équipement les plus importantes;

- quantités et propriétés des substances produites, entreposées ou manipulées sur place;

- résultats des essais de sécurité et données relatives à la sécurité pour les matières premières, les solvants, les catalyseurs, les produits intermédiaires et les produits secondaires, ainsi que les matières réactives et les produits de réaction;

- réactions secondaires et chimie de ces réactions;

- données obtenues grâce aux études des dangers;

- traitement des déchets (confinement et élimination).

" Le fichier de documentation sur le procédé et le dossier sur l'installation devraient être mis à jour régulièrement.

\section{Construction}

2.c.18 La direction d'une installation dangereuse devrait accorder une attention toute particulière à l'assurance de la qualité lors de la phase de construction d'un projet.

" Des vérifications et des inspections devraient être effectuées régulièrement pour contrôler la sécurité pendant la phase de construction, de façon à s'assurer que l'intégrité de la conception originale est bien respectée. Cela consiste à vérifier que les plans sont scrupuleusement suivis, que les exigences découlant des études de dangers sont intégralement appliquées et que l'équipement connexe est correctement installé. Cela consiste enfin à vérifier que les matériaux, les méthodes (comme les techniques de soudage) et les essais (comme ceux de pression/fuite) appropriés sont utilisés par un personnel suffisamment qualifié (employés ou contractants), et conformément aux normes reconnues.

" Toute modification à la conception originale d'une installation devrait être documentée, et ces modifications devraient se refléter dans l'assurance de la qualité et les examens en matière de sécurité avant la mise en service et le démarrage de l'installation.

" Les systèmes d'assurance de la qualité (AQ) peuvent fournir des outils utiles pour vérifier que l'équipement est bien conforme aux normes et aux autres exigences.

2.c.19 Des vérifications de la sécurité devraient également être effectuées aux stades de la mise en service et du démarrage d'un projet, de telle façon que le but de la conception soit pleinement atteint. Des essais fonctionnels devraient être effectués avec toutes les composantes ainsi que tous les dispositifs de contrôle et de sécurité, indispensables à la sécurité de l'installation.

2.c.20 Une entreprise devrait acheter son équipement uniquement auprès de fournisseurs connus, et, avant de l'utiliser, elle devrait inspecter l'équipement en bonne et due forme pour s'assurer qu'il est conforme aux spécifications de conception et aux exigences de 
sécurité. Les entreprises devraient partager entre elles l'information concernant la fiabilité des fournisseurs.

2.c.21 Pour la construction d'une installation dangereuse, une entreprise ne devrait travailler qu'avec des contractants pouvant lui donner entière satisfaction, c.-à-d. qui fournissent des services conformément à toutes les lois et réglementations applicables, ainsi qu'en conformité avec les normes et politiques pertinentes de l'entreprise en matière de sécurité, et ce de façon à ne pas augmenter le risque d'un accident mettant en cause des substances dangereuses. Les contractants devraient travailler selon les normes établies par la direction de l'installation et, dans la mesure du possible, sous la surveillance directe de la direction.

\section{d. EXPLOITATION}

\section{Procédures}

2.d.1 La direction devrait s'assurer que les dispositions organisationnelles appropriées pour la mise en oeuvre de la politique de sécurité de l'entreprise sont en place. La responsabilité hiérarchique pour la gestion de la sécurité dans l'entreprise et l'autorité pour procéder à des changements, ainsi que la responsabilité individuelle en matière de sécurité devraient être clairement définies. Les rôles et responsabilités de tous les employés (c.-à-d. la direction et les travailleurs, incluant les contractants) en matière de sécurité devraient être clairement déterminés.

2.d.2 La direction devrait s'assurer que chaque installation d'une entreprise dispose de procédures et d'instructions d'exploitation écrites et facilement accessibles, de façon à établir les conditions nécessaires pour répondre aux objectifs de conception de l'installation et maintenir son intégrité. Ces conditions devraient tenir compte des normes, des codes et des lignes directrices pertinents de telle façon que l'équipement, l'usine et les installations constituent un lieu de travail sûr dans des conditions d'exploitation aussi bien normales qu'exceptionnelles.

" Les procédures écrites devraient être bien comprises par tous les employés concernés (incluant les contractants). Il devrait y avoir des systèmes de formation pratique et théorique, d'examen et de surveillance pour faire en sorte que tous les employés connaissent, comprennent et observent en tout temps les procédures appropriées, et que ces procédures soient réexaminées régulièrement et mises à jour pour pouvoir tenir compte de toute modification importante dans la conception ou l'exploitation d'une usine.

" Les opérateurs, le personnel de maintenance et d'autres employés assumant des tâches liées à la sécurité dans l'installation devraient être appelés à participer à l'élaboration et à la mise à jour des procédures. Cela aide à faire en sorte que les procédures soient réalistes, faisables et appliquées de façon cohérente, et enfin donne un peu l'impression à ceux qui doivent suivre les procédures qu'ils en sont les « dépositaires ».

" Tous les employés visés devraient être au courant de toute modification apportée à l'usine. 
2.d.3 Des consignes et des dispositions devraient être prévues à toute installation dangereuse pour la prévention des incendies et des rejets de substances dangereuses. De plus, des dispositions appropriées devraient être prises pour la protection du personnel, des bâtiments et de l'équipement, ainsi que pour l'intervention (p. ex. pour combattre un incendie) en cas d'incendie ou de rejets de substances.

2.d.4 Il devrait y avoir des consignes pour s'assurer qu'il existe une protection efficace contre les accidents mettant en cause des substances dangereuses en cas de fonctionnement anormal. Les conditions de fonctionnement sont anormales, par exemple, dans les cas suivants: des instruments essentiels, des systèmes d'alarme ou de l'équipement d'urgence ne fonctionnent pas; demande de production inhabituelle (à court terme), lourde charge de travail en temps supplémentaire ou ralentissement de la production; contraintes au niveau des ressources (y compris la dotation et les ressources financières); mise hors service ou évacuation en cas d'urgence.

2.d.5 La direction devrait s'assurer que tous les employés possèdent, si nécessaire, un équipement de protection individuelle, et veiller à ce que cet équipement soit toujours en bon état.

" La direction devrait également s'assurer qu'une formation régulière est donnée pour l'utilisation de l'équipement de protection individuelle.

" Les employés devraient être responsables de l'utilisation de l'équipement de protection individuelle approprié, conformément aux consignes et aux politiques de sécurité.

" On devrait s'employer à concevoir les installations de façon à réduire au minimum les besoins en équipement de protection individuelle.

(Voir paragraphes 2.c.4-17 sur la conception, la planification et l'aménagement des installations)

2.d.6 Des consignes devraient être prévues pour les installations d'entreposage et celles où il y a présence de substances dangereuses, de façon à réduire au minimum les risques d'accident et notamment à prévenir la dégradation de ces substances, des emballages, des étiquettes ou d'autres marques utilisées dans ce contexte.

" Le cas échéant, le responsable de l'entrepôt doit veiller à ce que toutes les exigences légales pertinentes et les codes de pratique applicables pour l'entreposage de substances dangereuses soient rigoureusement observés.

" Pour prévenir les explosions et les incendies, il faudrait déterminer si les conditions d'entreposage (y compris, par exemple, la température et la pression) génèrent des risques particuliers. Il faudrait également éviter les sources potentielles d'allumage, comme la cigarette, le soudage et l'équipement d'emballage sous film rétractable. Tout l'équipement d'alimentation énergétique devrait être, si nécessaire, spécialement protégé.

" Un plan d'entreposage devrait être élaboré par le responsable de l'entrepôt, montrant la nature des substances dangereuses dans chaque partie de l'installation d'entreposage.

- le plan d'entreposage devrait être mis à la disposition des employés et des autorités locales compétentes (p. ex. services d'incendie); 
- l'information concernant les substances dangereuses conservées dans l'installation d'entreposage devrait être mise à jour régulièrement et rendue facilement accessible aux employés, aux représentants syndicaux et aux responsables des interventions en cas d'urgence.

" Lorsque l'entreposage relève d'une tierce partie (à l'extérieur de l'installation), le propriétaire des substances dangereuses (produits, matières premières et produits intermédiaires) devrait vérifier si l'entrepôt convient pour ces substances et si le responsable de ce dernier possède les qualifications voulues pour assumer de façon sûre les tâches d'entreposage. Cela pourrait vouloir dire que le propriétaire ou le fournisseur des substances ait à surveiller l'installation d'entreposage et à former les employés de l'installation hors site.

- le propriétaire ou le fournisseur des substances dangereuses entreposées devrait communiquer au responsable de l'entrepôt l'information nécessaire pour prévenir tout accident et intervenir de façon appropriée en cas d'accident, y compris l'information concernant la réaction et (ou) la décomposition des produits formés lors d'un incendie;

- à cet égard, le propriétaire ou le fournisseur devrait mettre à la disposition du responsable de l'entrepôt une fiche signalétique (FS) ou une fiche technique de chaque substance, de telle façon que le responsable de l'entrepôt puisse s'assurer que les propriétés physiques, chimiques, (éco)toxicologiques et autres, essentielles en cas d'accident, sont bien comprises par tous les employés concernés, travaillant à l'installation d'entreposage;

- une attention particulière devrait être accordée à l'étiquetage et au marquage appropriés des substances dangereuses, indiquant toute propriété dangereuse et les précautions adéquates à prendre;

- le propriétaire ou le fournisseur devrait s'assurer que les substances incompatibles sont bien séparées.

2.d.7 Des dispositions appropriées devraient être prévues pour préserver la sécurité à une installation dangereuse, réduire au minimum la possibilité de rejets délibérés à la suite, par exemple, d'actes de terrorisme, de sabotage ou de vandalisme, ou encore de vols de substances dangereuses. La direction de l'installation dangereuse devrait désigner les secteurs de l'installation dont l'accès devrait être limité ou contrôlé, et mettre en oeuvre des mesures pour assurer un contrôle permanent et interdire l'accès aux personnes non autorisées.

2.d.8 Un haut niveau de maintenance et d'efficacité opérationnelle devrait être maintenu aux installations dangereuses, y compris les entrepôts, car il existe une étroite corrélation entre ces fonctions et un bon rendement en matière de sécurité.

2.d.9 La direction devrait s'assurer que des procédures d'exploitation et des instructions pour la sécurité, écrites, pertinentes et acceptées accompagnent tout nouveau produit, procédé ou équipement avant son transfert d'un secteur à un autre (ou d'un propriétaire à un autre), de telle façon que les connaissances et l'expérience acquises en recherche, en développement, en usine pilote et à l'échelle de la production puissent être transmises elles aussi. Ce transfert devrait être officialisé par une attestation de passation ou un rapport d'autorisation appropriés, dûment signés.

" Cela s'appliquerait par exemple lorsqu'un produit ou procédé passe de la phase de la recherche ou du développement à la production réelle, ou lorsqu'il y a passation de la production la maintenance, ou retour de la maintenance à la production. 
" Les procédures d'exploitation et les instructions pour la sécurité devraient également être fournies chaque fois qu'il y a transfert d'installations ou de technologie.

\section{Personnel}

2.d.10 La direction devrait s'assurer que chaque opération est dotée d'un personnel suffisant et d'une façon qui permette d'exploiter les installations en toute sécurité en tout temps.

" On devrait s'assurer que les employés possèdent les qualifications leur permettant de s'acquitter de leurs tâches en toute sécurité (compte tenu des facteurs tant physiques que psychologiques), y compris les employés dont les activités sont largement sédentaires, comme les directeurs et les employés des postes de contrôle. À cet égard, aucune tâche ne devrait être confiée à un employé si cela compromet la sécurité de fonctionnement d'une installation.

" Il faut repérer les emplois qui ne conviennent pas aux handicapés, ni aux personnes à capacité réduite, ni aux femmes enceintes, ni aux jeunes, en raison du risque d'accident mettant en cause des substances dangereuses.

" Les employés et leurs représentants devraient participer au processus décisionnel concernant l'organisation de leurs activités et les besoins en dotation de l'installation, dans la mesure où ces éléments peuvent influer sur la sécurité.

" À cet égard, la direction devrait envisager des mécanismes pour l' " examen par des pairs » du rendement en matière de sécurité.

2.d.11 La direction devrait tout particulièrement s'assurer qu'il y a suffisamment de personnel et de supervision pendant la nuit et les fins de semaine, ainsi que pendant les périodes où la situation est difficile ou inhabituelle, et que le travail en temps supplémentaire ou des conditions de travail irrégulières n'augmentent pas le risque d'accidents mettant en cause des substances dangereuses.

" Dans la planification des calendriers de dotation, on devrait viser à éviter le stress chez le personnel ainsi que les charges de travail trop lourdes. Par exemple, les heures de travail et le temps des pauses devraient être compatibles avec les exigences en matière de sécurité. Le temps supplémentaire et le travail pendant les jours de repos par un employé quel qu'il soit devraient demeurer dans les limites du raisonnable. Toutes ces heures de travail anormales devraient être enregistrées dans un dossier pour faciliter le contrôle des heures travaillées.

" La direction devrait déterminer - pour y faire face - les besoins en dotation et en qualifications techniques spéciales pour les démarrages, les mises hors service, les conditions d'exploitation anormales ou particulières, les périodes correspondant à des demandes inhabituelles de production, à des contraintes au niveau des ressources ou à des situations d'urgence, ou encore à des situations qui pourraient être source de stress chez le personnel;

2.d.12 On devrait considérer si certaines tâches, en raison de leur relation avec la prévention des accidents, devraient faire l'objet de contrôles spécifiques par la direction; comme 
exemple, on peut citer l'obligation d'obtenir une autorisation spéciale, un permis de travail ou une licence pour des activités, comme la pressurisation de réservoirs ou le soudage.

2.d.13 La direction devrait prendre des mesures pour éviter toute situation dans laquelle des emplois, qui sont très importants pour la sécurité, deviennent trop routiniers ou trop limités au niveau des responsabilités.

2.d.14 Des politiques spécifiques concernant les activités individuelles qui pourraient mettre en péril la sécurité opérationnelle d'une installation - comme la cigarette, l'abus de certains produits, etc. - devraient être définies et incluses dans chaque contrat passé avec un employé ou dans les conditions d'embauche.

2.d.15 La direction ne devrait pas engager des entrepreneurs pour effectuer des tâches liées à l'exploitation d'une installation dangereuse si cela risque de nuire à la sécurité.

4 La direction devrait uniquement retenir les services d'entrepreneurs qui possèdent les qualifications pour mener à bien les travaux sous contrat selon les lois et règlements applicables, les politiques et normes de sécurité de l'entreprise et toute pratique complémentaire spécifique à ces travaux.

" Avant d'accorder un contrat, la direction devrait obtenir des données montrant que les entrepreneurs possèdent les qualifications pour s'acquitter de leurs tâches à un niveau de sécurité suffisamment élevé. La conformité aux lois, règlements, politiques et normes de sécurité en vigueur doit constituer une partie intégrante du contrat avec les entrepreneurs.

4 La direction devrait surveiller le rendement des contractants au niveau de la sécurité et, de façon générale, ces derniers devraient être assujettis aux mêmes systèmes de sécurité que le personnel de l'installation.

2.d.16 Les entrepreneurs engagés pour effectuer des travaux liés à l'exploitation d'une installation dangereuse devraient avoir, en matière de sécurité, des droits et des responsabilités équivalents à ceux du personnel de l'installation. Des mesures spéciales doivent, si nécessaire, être mises en place pour s'assurer que les contractants sont bien informés au sujet des dangers inhérents à l'exploitation d'installations dangereuses. Il faudrait leur fournir l'information sur la sécurité propre au site.

2.d.17 Il faudrait vérifier si les réductions dans le niveau de dotation, tant chez les travailleurs (comme les opérateurs) que dans la direction, pourraient avoir des effets nuisibles sur la sécurité.

" Il s'agit là d'une question importante, vu que les conditions économiques peuvent conduire à une réduction du nombre d'employés et à des changements dans les structures de l'entreprise.

" Des réductions dans le niveau de dotation ne nuisent pas nécessairement à la sécurité, car il y a d'autres facteurs qui interviennent, comme la conception, la gestion et l'exploitation. Cependant, il est possible que des coupures dans le personnel entraînent une baisse dans les communications relatives à la sécurité, un manque de coordination entre les politiques et la pratique, un plus grand stress, et 
moins de temps pour la formation, les inspections spontanées et la période de repos entre deux quarts de travail. Elles peuvent également se traduire par une perte au niveau de l'expérience et par un nombre plus grand d'opérateurs travaillant seuls, plutôt qu'avec des collègues.

2.d.18 Le rendement en matière de sécurité devrait être considéré comme une composante essentielle du rendement global de tout directeur ou autre employé, et il devrait être réexaminé régulièrement. Le rôle des directeurs et des travailleurs (à tous les niveaux) concernant la sécurité devrait être clairement défini, de telle façon que le rendement en matière de sécurité puisse faire l'objet d'un suivi et d'un réexamen appropriés.

2.d.19 La coopération entre la direction et les travailleurs, à tous les niveaux, est essentielle si on veut exploiter une installation dangereuse en toute sécurité.

" La gestion devrait encourager les travailleurs à assumer leurs rôles et responsabilités et leur faciliter la tâche.

" Les travailleurs peuvent tirer parti de l'expérience et du support de leurs syndicats, de leurs confédérations et de leurs organisations internationales pour les aider.

2.d.20 La direction d'une installation dangereuse devrait prendre toutes les mesures raisonnables pour informer les employés sur place, y compris les contractants, au sujet des substances dangereuses auxquelles ils peuvent être exposés. L'information appropriée sur les dangers (notamment les niveaux d'exposition lors de situations d'urgence) et sur les procédures à suivre pour la manipulation adéquate de toutes les substances de l'installation (incluant celles utilisées, préparées comme produits intermédiaires, entreposées ou disponibles pour la vente) devrait être réunie, mise à jour et diffusée largement dans une (des) langue(s) que tous les employés peuvent comprendre.

\section{(Voir paragraphes 2.d.25-33 sur la communication interne et paragraphes 2.d.34-41 sur la formation pratique et théorique)}

2.d.21 On devrait s'employer à s'assurer que les employés sont bien informés au sujet des activités concernant leur milieu de travail et y participent, notamment au niveau de la maintenance, des essais et de l'étalonnage. De plus, ils devraient recevoir une formation et être mis à contribution dans des activités connexes, comme la conception des aires de travail, les évaluations des risques et les audits des installations.

2.d.22 La planification pour le perfectionnement et la rotation du personnel devraient toujours se situer dans le cadre du respect des exigences relatives à la sécurité opérationnelle. Cela s'applique aux employés à tous les niveaux, y compris la direction.

2.d.23 À l'intérieur de l'entreprise, on devrait disposer d'un nombre suffisant d'employés spécialisés en sécurité. Ils devraient demeurer impartiaux et indépendants de la gestion hiérarchique, servir comme experts-conseils et, à ce titre, jouer le rôle de la conscience de l'entreprise en matière de sécurité.

" À cet égard, les spécialistes en sécurité devraient :

- disposer de l'autorité nécessaire pour assumer leurs responsabilités, et avoir visiblement le support de la direction; 
- dialoguer avec les employés à tous les niveaux de l'entreprise, et jouir de leur estime;

- être compétents du point de vue technique soit par formation spécialisée, soit par expérience (de préférence les deux);

- avoir de bonnes relations interpersonnelles et de la facilité pour la communication.

" Le nombre de spécialistes en sécurité devrait correspondre à la taille, à la technologie et à la complexité de l'entreprise.

" La direction devrait considérer la rotation des employés entre la gestion hiérarchique et les fonctions liées à la sécurité de façon à mieux faire comprendre les problèmes inhérents à la sécurité, à trouver de meilleures solutions à ces problèmes, et enfin à renforcer la culture de la sécurité à l'intérieur de l'entreprise.

2.d.24 Chaque employé devrait être tenu de suivre les procédures édictées par la direction, et de prendre raisonnablement soin de sa sécurité personnelle ainsi que de celle des autres qui pourraient être touchés par ses actes ou ses négligences au travail.

" Chaque employé devrait aider les autres à s'acquitter en toute sécurité de leurs tâches et à collaborer activement avec la direction pour appliquer les consignes et les dispositions relatives à la sécurité.

" Il est important que les employés à tous les niveaux reçoivent la formation et les ressources dont ils ont besoin pour mener à bien leurs tâches et, en même temps, qu'ils assument la responsabilité de leurs tâches (et en soient tenus comptables), que ce soit à titre individuel ou comme membres d'une équipe.

" Même si chaque individu est responsable de son propre rendement en matière de sécurité, c'est l'entreprise qui doit créer les conditions qui permettront à chaque individu d'agir de façon responsable et efficiente.

4 L'expérience semble montrer que la sécurité se trouve améliorée lorsqu'une organisation confie à ses employés des responsabilités dans une atmosphère de confiance et leur fournit les outils nécessaires pour travailler et prendre des décisions.

\section{Communication interne}

2.d.25 Des voies efficaces de communication bidirectionnelle pour le transfert de l'information concernant la sécurité entre la direction et les travailleurs devraient être mises sur pied aux installations dangereuses.

" Cela aidera à créer et à maintenir un haut niveau de motivation chez tous les employés pour exploiter l'installation de façon sûre.

4. Il faut prendre soin de s'assurer que les liaisons importantes de communication ne sont pas coupées du fait, par exemple, de différences de langue ou d'une présomption voulant que certains employés ou contractants soient négligents ou ne soient pas capables de comprendre la pertinence de certains faits. 
2.d.26 Les voies régulières de communication devraient être renforcées par la création de comités de la sécurité devant servir de mécanisme officiel pour la consultation entre la direction, les travailleurs et leurs représentants en sécurité. Les comités de la sécurité devraient favoriser - mais non remplacer - la communication directe en matière de sécurité entre la direction et les travailleurs, ainsi qu'entre les niveaux de responsabilité individuelle et ceux de la gestion hiérarchique. L'emploi de ces comités permet de tirer le maximum d'avantages de l'expérience et des connaissances pratiques des employés, et favorise la confiance mutuelle grâce aux mesures prises pour améliorer la sécurité.

" Les comités de la sécurité devraient fonctionner à divers niveaux de l'entreprise. Ces comités pourraient, selon la taille de l'entreprise, être composés de :

- travailleurs de divers niveaux (dont les représentants en matière de sécurité lorsqu'il y en a);

- directeurs habilités à mettre en oeuvre les recommandations du comité;

- spécialistes de la sécurité;

- contractants, le cas échéant.

" Les membres du Comité de la sécurité devraient recevoir une formation en sécurité et, si nécessaire, des conseils de spécialistes.

Des ressources devraient être mises à la disposition du Comité de la sécurité pour lui permettre d'agir.

4 La direction devrait prendre ses décisions en fonction des recommandations du Comité de la sécurité; la responsabilité ultime pour la sécurité demeure du ressort de la direction.

Des membres du Comité de la sécurité ne devraient perdre aucun revenu en raison du temps consacré à des activités au sein du comité.

2.d.27 En plus du Comité de la sécurité constitué dans une installation dangereuse, la mise en place de mécanismes similaires aux niveaux de l'entreprise, du secteur ou encore à l'échelle nationale ou internationale peut se révéler utile pour faciliter la diffusion de l'information concernant la sécurité et étayer les processus décisionnels en matière de sécurité.

2.d.28 Les représentants en sécurité à l'échelle de l'usine devraient bénéficier de support dans leur travail. Ces représentants désignés par les travailleurs examinent avec la direction les questions liées à la sécurité. Les représentants en sécurité devraient recevoir une formation spéciale, propre à leurs fonctions.

2.d.29 Aucune mesure disciplinaire contre un employé ne devrait être prise si celui-ci se plaint de bonne foi auprès des autorités compétentes ou d'employés assumant des responsabilités en matière de sécurité, au sujet de ce qu'il considère comme une violation de la réglementation ou une carence au niveau des mesures prises aux fins de la sécurité. La direction doit encourager ce type de démarche si on veut instaurer le climat de « transparence » nécessaire pour les questions de sécurité.

2.d.30 Un employé devrait avoir le droit de refuser d'effectuer toute tâche dans laquelle il juge qu'il y a un risque non justifié d'accident mettant en cause des substances dangereuses. 
" L'employé devrait immédiatement signaler à la direction les raisons pour lesquelles il refuse d'assumer cette tâche.

"Dans certains cas, un employé ou un représentant en sécurité, s'il y en a un, pourrait interrompre des activités dangereuses en adoptant la démarche la plus sûre possible lorsqu'il juge que ces activités peuvent constituer un danger imminent et grave pour la sécurité.

2.d.31 Les employés devraient immédiatement faire rapport à la direction sur toute situation qui, selon eux, pourrait s'écarter des conditions normales de fonctionnement, et notamment les situations qui pourraient entraîner un accident mettant en cause des substances dangereuses.

"La direction devrait enquêter sur ces rapports.

" Tout employé devrait avoir le droit de signaler des conditions non sûres aux pouvoirs publics compétents.

2.d.32 Aucun employé ne devrait être défavorisé en raison d'initiatives comme celles décrites dans les deux paragraphes précédents.

2.d.33 La direction des installations dangereuses devrait fournir l'information et l'aide technologiques concernant la sécurité en matière de substances dangereuses aux contractants, distributeurs, transporteurs, clients et utilisateurs en aval, ainsi qu'aux employés.

\section{Formation pratique et théorique}

- 2.d.34 La direction devrait prendre toutes les mesures raisonnables pour s'assurer que tous ceux qui travaillent dans une installation dangereuse, y compris les employés temporaires et les contractants, reçoivent une formation pratique et théorique appropriée et qu'ils possèdent les compétences voulues pour s'acquitter de leurs tâches dans des conditions tant normales qu'inhabituelles.

4 La sensibilisation et la formation doivent porter sur les domaines suivants :

- identification des dangers, évaluation des risques, mesures correctives appropriées pour faire face aux problèmes de sécurité;

- prévention et atténuation des risques;

- mesures qui devraient être prises en cas de situation inhabituelle ou d'urgence;

- mesures correctives apportées aux méthodes de manutention des produits;

- dangers particuliers propres à une tâche donnée.

" La formation en sécurité devrait faire partie de la formation initiale donnée à tous les nouveaux employés pour les sensibiliser aux questions de sécurité et les engager à y faire face. Il devrait également y avoir de la formation régulière sous forme de suivi. Pendant les périodes de ralentissement des opérations ou si les circonstances le dictent, on devrait considérer la possibilité d'utiliser le temps libre des employés pour l'enseignement et la formation.

" La formation devrait être structurée de façon à développer chez chaque employé les capacités lui permettant de s'acquitter des tâches qui lui ont été confiées, et elle devrait être assez vaste pour permettre aux employés de comprendre le 
fonctionnement de l'installation, de l'équipement et des procédés ainsi que les possibilités de situation anormale. L'approche en matière de formation pratique et théorique devrait susciter le haut niveau de sensibilisation nécessaire non seulement pour prévenir les accidents, mais également pour faire face rapidement et efficacement à des situations anormales.

"Des dispositions devraient être prises pour s'assurer que les besoins en formation spécialisée à tous les niveaux sont clairement établis et satisfaits de façon adéquate.

" On devrait encourager et former tous les employés à examiner les tâches qui leur sont assignées et à la façon dont ils peuvent les accomplir avec une sécurité optimale, plutôt que de les exécuter mécaniquement. La formation devrait montrer clairement non seulement ce que les employés sont tenus d'accomplir, mais également pourquoi certaines activités sont indispensables pour la sécurité. À cet égard, la formation devrait inciter les employés à faire part, si nécessaire, de leurs préoccupations en matière de sécurité (qu'il s'agisse de questions techniques ou de problèmes de gestion).

" Les travailleurs et leurs représentants devraient être appelés à participer à l'élaboration des programmes d'enseignement et de formation.

2.d.35 Dans l'élaboration et la mise en oeuvre des programmes, on devrait considérer les méthodes de formation les plus efficaces pour des situations particulières, y compris la formation pour les opérations quotidiennes et pour faire face à des situations inhabituelles ou d'urgence.

" Parmi les différentes méthodes de formation, on peut citer les suivantes : formation d'un opérateur par un autre; systèmes en ligne; modèles de simulation électroniques. La formation à l'aide de simulateurs permet de se familiariser avec l'application du diagnostic et des mesures correctives pour l'exploitation des systèmes automatisés modernes.

" On devrait, si possible, considérer la formation des employés en groupes plutôt qu'individuellement, car ce type de formation peut représenter un moyen efficace pour insuffler aux employés une bonne attitude en matière de sécurité, développer chez eux un comportement positif en groupe, et enfin augmenter la capacité des membres du groupe à prévoir les problèmes potentiels de sécurité et à trouver des solutions.

" Il devrait y avoir des activités de formation conjointe pour les gestionnaires et les travailleurs afin de les aider à mieux comprendre leurs rôles et responsabilités mutuels.

2.d.36 Les directeurs ont une obligation personnelle de se tenir au courant des normes et des risques en matière de sécurité. Ils devraient connaitre et pleinement comprendre les propriétés et le comportement des substances dangereuses utilisées ainsi que les limitations de l'équipement et de la technologie.

"Les directeurs devraient avoir la capacité de mettre en oeuvre les mesures à prendre en cas d'urgence. 
4. Chaque directeur devrait s'assurer que les personnes de son équipe savent comment accomplir en toute sécurité les tâches qui leur sont confiées et comment maintenir un haut niveau de sensibilisation à la sécurité. Pour réaliser cela, chaque superviseur devrait recevoir une formation dans les domaines suivants: techniques de communication, initiative en matière de sécurité, enquête sur les accidents et production de rapports, analyses de la sécurité et de la santé, organisation de réunions sur la sécurité.

2.d.37 Des exercices devraient être effectués assez fréquemment, de telle façon que les opérateurs puissent bien analyser les situations d'urgence et réagir adéquatement. La perception fonctionnelle, particulièrement en ce qui concerne la prise de décisions dans des situations d'urgence, constitue un facteur important pour la sécurité opérationnelle. La perception peut être complexe et se baser sur l'information acquise antérieurement et la connaissance des systèmes.

2.d.38 La nature des besoins en formation pour la sécurité devrait être analysée régulièrement; de plus, les programmes de formation devraient faire l'objet d'un suivi et d'une évaluation de leur efficacité, pour être éventuellement révisés.

" La formation pratique et théorique devrait être modifiée pour refléter les changements dans les procédés utilisés, la technologie appliquée et les procédures suivies à une installation.

" Ce processus d'évaluation et de révision est particulièrement important lorsque surviennent des changements, comme pendant les périodes où des employés, y compris les directeurs et les superviseurs, sont assignés à une installation nouvelle ou différente.

" Les travailleurs et leurs représentants devraient être appelés à participer à l'expérimentation et à l'évaluation des programmes d'enseignement et de formation, et à leur révision ultérieure.

2.d.39 La direction devrait tenir compte d'éventuelles différences au niveau de la langue, de telle façon que les employés puissent comprendre le contenu de l'enseignement et de la formation, et communiquer avec leur collègues.

" Le cas échéant, la formation pratique et théorique devrait être disponible dans des langues autres que la première langue utilisée à l'installation, comme dans les cas où il y a des employés étrangers ou lorsque l'installation est située dans une région multilingue.

" Lorsque les employés parlent différentes langues, la direction devrait offrir des cours de langue de telle façon que l'on dispose d'une langue de communication commune pour faire fonctionner l'installation en toute sécurité et faire face aux situations d'urgence.

2.d.40 Des dossiers devraient être tenus et mis à jour concernant la formation pratique et théorique en sécurité reçue par tout membre du personnel, y compris les directeurs et les contractants. 
2.d.41 L'enseignement et la formation devraient être considérés comme faisant partie des tâches de l'employé aux fins du calcul du temps de travail et du salaire.

\section{Facteurs humains}

2.d.42 Pour la prévention des incidents aux installations dangereuses et pour faire face aux situations anormales, une attention particulière devrait être accordée au rôle des « facteurs humains ».

D À cet égard, il faudrait être conscient du fait que l'homme peut parfois se tromper et que la majorité des accidents sont, d'une façon ou d'une autre, attribuables à une erreur humaine, comme une action ou une inaction, qui involontairement, active les points faibles dans l'équipement, les procédures, les systèmes et (ou) les organisations.

》 L'expression «facteur humain » est souvent utilisée dans un contexte négatif (on a tendance à l'assimiler à erreur humaine). Cependant, l'homme est souvent le seul moyen qui existe pour faire face efficacement à une situation anormale, car il a la faculté de raisonner et de transcender les réactions automatiques des machines. L'homme a la capacité d'anticiper l'action, d'assimiler une information complexe et floue, et enfin d'affronter des situations inhabituelles grâce à ses connaissances et à son expérience. Ainsi, un employé peut redresser une situation potentiellement peu sûre s'il dispose d'assez de renseignements et de formation, et si le lieu de travail est conçu de façon à lui permettre de prendre des mesures correctives.

2.d.43 On devrait tenir compte du « facteur humain » à tous les stades propres à une installation dangereuse, et notamment aux suivantes : conception, construction, identification des dangers et évaluation du risque, exploitation, formation pratique et théorique, maintenance, mise hors service et fermeture.

Le facteur humain, incluant les aspects tant positifs que négatifs du comportement humain, s'applique à tous ceux qui sont employés dans une installation dangereuse (c.-à-d. directeurs et travailleurs, y compris les contractants).

" Les lieux de travail, y compris les outils et l'équipement connexes, devraient être conçus en fonction des principes ergonomiques et de l'interface employé/machine, de telle façon que ces lieux cadrent avec les capacités et les limitations physiques et cognitives de l'employé.

\ Les tâches qui peuvent nuire à la sécurité de l'exploitation d'une installation devraient être analysées de façon à pouvoir confier à chaque employé des fonctions adaptées à ses capacités physiques et psychologiques, et à faire en sorte que les employés n'aient pas une charge de travail trop lourde et ne soient pas soumis à un stress excessif.

\section{(Voir section 2.c sur l'implantation, la conception et la construction)}

2.d.44 Les employés devraient être encouragés à partager leur expérience afin de réduire le risque d'erreur humaine.

* Cela peut être réalisé, par exemple, en organisant des ateliers sur la sécurité, des discussions sur les quasi-accidents et d'autres groupes de discussions, ainsi que par 
la visite et l'inspection des lieux de travail par les employés et, le cas échéant, par les représentants en sécurité.

" L'expérience liée aux erreurs humaines devrait également être partagée entre les différentes entreprises et, dans la mesure du possible, avec les pouvoirs publics.

\section{(Voir paragraphes 2.d.25 - 33 sur la communication interne)}

2.d.45 Des précautions spéciales devraient être prises pour éviter les erreurs humaines pouvant entraîner des accidents pendant des périodes d'exploitation inhabituelle ou lorsque les employés peuvent subir des contraintes (p.ex. en cas de demande inhabituelle de production à court terme, de travail supplémentaire excessif, de ralentissement dans la production, ou enfin de restrictions au niveau des ressources). La direction devrait démontrer que les considérations de sécurité ont précédence sur les autres considérations. La contrainte s'exerçant sur la sécurité pourrait résulter de la pression sur les employés individuellement ou sur les groupes d'employés ou encore sur toute l'entreprise (par exemple pour augmenter la production ou réduire les coûts).

\section{(Voir paragraphes 2.d.10 - 24 sur le personnel)}

2.d.46 Des précautions spéciales devraient être prises pendant et après les modifications et la maintenance, lors de la fermeture et du démarrage ainsi qu'après les interruptions de service, du fait que les erreurs ont tendance à augmenter pendant et après ces périodes.

\section{(Voir sections 2.e sur la maintenance et les réparations et 2.f sur les modifications)}

\section{e. MAINTENANCE ET RÉPARATIONS}

2.e.1 La direction d'une installation dangereuse devrait préparer des programmes pour la maintenance régulière, l'inspection et l'essai de l'équipement de façon à s'assurer qu'il est en tout temps adapté aux fins pour lesquelles il a été conçu.

" Une attention particulière devrait être portée pendant les périodes de maintenance et de réparations, vu que le risque d'accident est plus élevé lors de ces périodes.

" Les programmes de maintenance doivent être rigoureusement respectés, et réexaminés régulièrement pour s'assurer qu'ils continuent à répondre aux exigences de sécurité.

" Des normes et procédures de maintenance et de réparation devraient être élaborées pour aider à garantir la sécurité de chaque opération, et toutes les tâches devraient être effectuées selon ces procédures.

"Des procédures devraient exister pour les dispositifs de verrouillage et les permis à chaud.

4 Les programmes de maintenance devraient tenir compte de l'information obtenue dans le cadre de l'identification des dangers et de l'évaluation des risques. 
" La direction devrait s'assurer que tous les contractants chargés de la maintenance ou des réparations connaissent et appliquent toutes les normes et procédures pertinentes.

" Des dossiers devraient être tenus sur tous les travaux de maintenance liés à la sécurité; des procédures d'examen de l'équipement et d'assurance de la fiabilité devraient être élaborées.

" Des dossiers devraient être tenus sur tout défaut constaté lors de la maintenance de l'équipement, qui pourrait nuire matériellement à la sécurité; des mesures devraient être prises rapidement pour corriger le défaut.

"Il devrait y avoir des procédures pour la mise hors service et le redémarrage en toute sécurité de l'installation, par exemple pendant la maintenance de l'équipement. On devrait tout faire pour éviter les causes possibles de risques, notamment les problèmes de communication et de responsabilité partagée; cela peut être particulièrement préoccupant si des contractants sont mis à contribution (ils ne sont peut-être pas pleinement au courant des détails concernant le fonctionnement, les politiques et les procédures propres à une installation).

2.e.2 La direction locale de chaque installation dangereuse devrait inspecter et entretenir régulièrement les systèmes d'alarme, l'équipement de protection et d'urgence ainsi que tous les dispositifs essentiels pour l'arrêt sans heurts des opérations, en collaboration (le cas échéant) avec les pouvoirs publics concernés.

\section{f. MODIFICATIONS (techniques et organisationnelles)}

2.f.1 La direction d'une installation dangereuse devrait introduire des procédures officielles pour faire en sorte qu'aucune modification (ou réparation) à l'usine, à l'équipement, aux procédés, aux logiciels (incluant les systèmes de commande automatisés), à l'installation ou encore aux procédures ne compromette la sécurité.

" Les procédures de modification devraient s'appliquer aussi bien aux changements permanents que temporaires, et elles devraient être basées sur la documentation industrielle appropriée et mise à jour et, le cas échéant, sur une inspection physique de l'installation.

" Tous les projets de modifications à une installation dangereuse devraient être enregistrés, documentés et évalués, de façon à pouvoir procéder aux études nécessaires sur les dangers, apporter les modifications appropriées à la conception, et enfin organiser et enregistrer correctement ces dernières.

- les projets visant des modifications significatives devraient être examinés par des techniciens compétents, indépendants de ceux directement à l'origine des projets;

- le niveau d'approbation par la direction, requis pour une modification, devrait être fondé sur le niveau de risque connexe;

- les superviseurs qui ont le mandat d'apporter une modification (par exemple, pour changer un procédé de fabrication ou une instruction opérationnelle), devraient être pleinement au courant des dangers connexes et devraient consulter le (les) spécialiste(s) compétent(s) pertinent(s) avant de procéder à ce changement. 
" Toute modification majeure devrait être soumise aux mêmes exigences de notification et de déclaration que les nouvelles installations.

2.f.2 Dans les cas où des changements apportés à un procédé pourraient nuire à la sécurité par exemple, utilisation de matières premières différentes, changements des conditions, augmentation de la taille d'un lot, emploi d'un équipement plus grand ou différent l'analyse initiale du danger devrait être réexaminée et il faudrait aussi compléter le fichier de documentation sur le procédé ou le dossier sur l'installation.

4 Il devrait également y avoir des procédures pour s'assurer que les changements au niveau de la gestion, chez les travailleurs ou dans l'organisation ne compromettent pas la sécurité (y compris, par exemple, les changements dans la structure ou le financement de l'entreprise, la réduction des effectifs, la sous-traitance d'une partie de la production). Ces changements devraient enclencher des procédures de réexamen pour s'assurer que la sécurité n'a pas subi de recul).

" Des techniques devraient être mises au point pour évaluer comment une série de changements mineurs dans l'installation peuvent globalement nuire à la sécurité, et pour déterminer ce qu'il serait possible de faire pour atténuer toute croissance du potentiel d'accidents.

2.f.3 Des procédures devraient être en place pour la remise en service d'une installation après modification, réparation et (ou) remise en état d'une usine, d'équipement ou de logiciels. Ces procédures devraient comporter des essais de fonctionnement et des vérifications de la sécurité pour s'assurer de l'intégrité de l'installation. Les essais de fonctionnement devraient être effectués en présence d'un directeur responsable de l'exploitation de l'installation. Le directeur devrait être tenu d'approuver officiellement la remise en service de l'installation.

2.f.4 Tous les employés concernés devraient être au courant de toute modification apportée à l'installation. Toute modification importante au niveau de l'usine, des procédés, de l'aménagement, du personnel, des logiciels ou d'autres aspects qui peuvent nuire à la sécurité devrait enclencher un réexamen des pratiques de formation afin de déterminer s'il y a des besoins additionnels en formation.

2.f.5 Les contractants ayant participé à une modification quelle qu'elle soit devraient être soumis aux mêmes procédures, y compris les mêmes exigences d'enregistrement, de déclaration et d'évaluation que le personnel de l'installation. Des procédures devraient être en place pour que les contractants ayant participé à des modifications puissent faire part à la direction de toute préoccupation concernant la sécurité.

\section{g. EXAMEN ET ÉVALUATION DE LA PERFORMANCE EN MATIÈRE DE GESTION DE LA SÉCURITÉ}

(Voir également section 3.c, traitant de l'examen et de l'évaluation de la performance en matière de sécurité par les pouvoirs publics)

2.g.1 L'industrie devrait organiser la surveillance (p. ex. par des audits) des programmes afin de faciliter l'amélioration permanente de la sécurité aux installations dangereuses. 
" La gamme des activités de surveillance par l'industrie comprend l'évaluation permanente de la gestion relative à l'environnement, à la santé et à la sécurité à l'échelle de l'installation, l'auto-évaluation par l'installation, les audits de l'installation par l'entreprise, et enfin les audits ou inspections par des tierces parties.

" Ces activités permettent de procéder à des vérifications pour s'assurer que les éléments d'un système de gestion de la sécurité sont en place et sont appliqués de façon appropriée pour atteindre les buts et les objectifs spécifiés.

2.g.2 L'approche adoptée pour la surveillance devrait être systématique. À cet égard, un plan de surveillance devrait être élaboré à chaque installation, «appartenant » à la direction locale et mis en oeuvre en premier lieu par cette dernière, et enfin offrant une certaine souplesse pour ne pas devenir routinière.

" Le plan de surveillance devrait inclure des réexamens réguliers, avec la participation des travailleurs et de leurs représentants au lieu de travail, des vérifications détaillées à intervalles réguliers d'activités et de procédures spécifiques, ainsi qu'un audit global de la performance.

" Le plan de surveillance d'une installation devrait former la base d'une série hiérarchique de rapports annuels d'assurance de la sécurité, depuis le directeur responsable d'une installation jusqu'aux directeurs divisionnaires / commerciaux / opérationnels et, ultérieurement, jusqu'au directeur général de l'entreprise.

" Comme le montrent les évaluations des risques, on devrait mettre l'accent sur les aspects qui sont vitaux pour la sécurité d'une installation donnée. Certains aspects généraux devront être couverts par le processus de surveillance, notamment les suivants: organisation et gestion; formation; intégrité de l'usine; protection et prévention contre l'incendie; enquête et déclaration concernant les accidents ou les situations dangereuses; consignes en cas d'urgence.

" Le niveau potentiel de risque devrait être un facteur important lorsqu'on détermine la fréquence de la surveillance.

2.g.3 Toute la surveillance devrait être considérée comme un circuit fermé avec "rétroaction » (c.-à-d. planifier, exécuter, vérifier, intervenir), conçu pour apporter des améliorations permanentes (tout en sachant que les programmes de surveillance peuvent différer au niveau des objectifs et des méthodes). Il y a certains éléments communs à la réussite de tous les programmes de surveillance (c.-à-d. des audits et des inspections). Plus précisément, ils devraient tous comporter les éléments suivants :

- objectifs clairement définis;

- portée bien délimitée, car si l'objectif recherché est trop vaste, il sera peut-être difficile à atteindre;

- calendrier (incluant, p. ex. un plan d'action dans des délais fixés);

- expert(s) approprié(s), formés et qualifiés pour les tâches et objectifs particuliers;

- analyse de la documentation pertinente et entrevues avec le personnel de base (notamment les opérateurs industriels);

- détection des carences et recensement des pratiques appropriées;

- rapport officiel sur les résultats; 
- examen par la direction, visant à définir clairement les responsabilités, un échéancier pour le suivi et des moyens pour s'assurer que les mesures pertinentes sont prises;

- faits montrant que les mesures de suivi ont été prises.

2.g.4 On devrait s'employer à améliorer la transparence lors des audits, notamment à rendre publics les politiques, les programmes et les résultats. Cela peut aider à établir et à maintenir la confiance parmi les intervenants (pouvoirs publics, industrie, employés, population, etc.). Les faits montrent qu'une plus grande sensibilisation du public aux risques conduit indirectement à l'amélioration de la sécurité.

" L'industrie devrait déployer des efforts concertés pour rendre publique l'information pertinente sous une forme facile à comprendre, et susciter des occasions de dialogue entre les intervenants (industrie, pouvoirs publics et population).

"Un énoncé sur la performance en santé et sécurité de l'entreprise devrait faire partie du rapport annuel préparé à l'intention des actionnaires et des employés.

\section{(Voir l'encadré texte sur la GRI [Global Reporting Initiative] à la fin du chapitre 2)}

" Il faut aider le public à mieux comprendre la nature du «risque » et les risques présentés par les installations dangereuses.

2.g.5 On devrait envisager de faire participer les représentants de la communauté (y compris, par exemple, le personnel d'intervention en cas d'urgence) aux audits. Cela pourrait aider à améliorer le niveau de confiance entre ceux qui sont responsables de l'exploitation d'une installation dangereuse et la communauté locale.

2.g.6 L'industrie devrait partager l'information - à l'intérieur du pays et à l'échelle internationale - sur les méthodes et les outils utilisés pour les inspections et les outils, ainsi que sur les résultats d'inspections et d'audits spécifiques. On devrait s'employer à promouvoir systématiquement ce type d'activités et trouver des mécanismes qui pourraient faciliter le partage de l'information.

2.g.7 On devrait chercher à utiliser davantage d'indicateurs de performance majeurs comme moyen pour mesurer la sécurité et déterminer si les mesures prises permettent réellement de réduire le risque. De plus, ces indicateurs pourraient aider à canaliser les audits et les inspections vers des secteurs à priorité maximale 9 .

2.g.8 Toutes les entreprises devraient mettre sur pied des programmes de surveillance, constitués de plusieurs niveaux d'audits, afin de vérifier divers systèmes techniques et de gestion à l'intérieur d'une installation. Ces programmes de surveillance permettent à la direction de réexaminer son fonctionnement et ainsi veiller à ce que qu'aucun risque non encore détecté antérieurement n'ait été introduit et qu'il y ait le niveau requis de conformité aux lois, normes, codes et lignes directrices, tant à l'échelle nationale $\mathrm{qu}^{\prime}$ internationale, ainsi qu'aux propres exigences et Principes directeurs de l'entreprise. En plus de détecter toute carence ou tout problème potentiel dans l'installation, l'audit devrait également reconnaître les mesures couronnées de succès, les leçons tirées de l'expérience et les améliorations réalisées en matière de sécurité.

" Un audit au niveau de l'usine peut fournir des données détaillées sur les activités quotidiennes des opérateurs de procédés ainsi que de l'information via ceux qui sont 
familiers avec les systèmes, alors qu'un audit conduit par l'administration centrale ou une tierce partie peut détecter des problèmes potentiels pouvant passer inaperçus aux yeux de ceux qui sont directement engagés dans l'exploitation, repérer les problèmes potentiels dans la structure organisationnelle de l'entreprise ou dans son système d'audits, et aider à trouver de nouvelles idées pour des améliorations.

"Les audits peuvent révéler l'existence de besoins additionnels en normes, en matériel informatique et (ou) en procédures. De plus, ils peuvent fournir : un outil de gestion ou de stratégie pour définir les priorités aux fins de l'attribution des ressources; un mécanisme pour partager l'information et l'expérience sur les meilleures pratiques; un support pour aider la gestion hiérarchique à assumer ses responsabilités; un moyen pour détecter les améliorations en matière de sécurité; un apport au dialogue avec les pouvoirs publics et la population; un mécanisme permettant de déterminer si les politiques internes ou les exigences légales sont respectées.

" Les audits devraient être l'un des éléments du système de gestion de la sécurité $d^{\prime}$ une entreprise.

" Les audits constituent également la base pour réexaminer la validité du système de gestion de la sécurité et, le cas échéant, pour le revoir afin de vérifier son bien-fondé et sa conformité aux meilleures pratiques.

2.g.9 La direction devrait, le cas échéant, utiliser des experts indépendants pour surveiller les installations dangereuses. L'emploi d'experts d'une tierce partie peut, dans certains cas, constituer un moyen extrêmement utile pour améliorer le rendement en matière de sécurité en proposant un point de vue indépendant.

D Pour la mise sur pied des programmes de surveillance, on devrait considérer la possibilité de confier certains audits à une tierce partie indépendante, selon l'objet et la nature de l'audit.

" Les compagnies d'assurance pourraient fournir un service utile à cet égard, particulièrement dans le cas des petites et moyennes entreprises.

2.g.10 Les audits devraient comprendre des entrevues avec les employés occupant des postes clés, au niveau tant des travailleurs que de la direction. Ces entrevues aident à vérifier que les employés comprennent les procédures opérationnelles et qu'ils accomplissent leurs tâches conformément à ces procédures. Elles permettent également de mieux savoir si les employés sont conscients des dangers en cause et de leur rôle pour y faire face. De plus, les entrevues aident à déterminer si l'information circule efficacement dans l'entreprise (c.-à-d. si l'échange d'information se fait dans les deux sens) et si la direction peut apprendre de l'expérience des employés.

2.g.11 Une équipe d'audit devrait être constituée pour chaque processus d'audit.

" Les membres d'une équipe d'audit devraient être choisis en fonction des besoins et des objectifs de l'audit et devraient, le cas échéant, comprendre des représentants de diverses fonctions, comme l'exploitation, la maintenance, l'ingénierie, la sécurité, la santé et l'environnement. 
" Les membres de l'équipe d'audit devraient avoir de l'expérience pratique et une formation adéquate pour détecter les dangers potentiels à l'installation et enregistrer les résultats. Les programmes de formation devraient être conçus pour aider les vérificateurs à déterminer à la fois les carences et les bonnes pratiques que l'on retrouve à l'installation.

2.g.12 Les membres de l'équipe d'audit devraient être appelés à participer à l'élaboration des programmes d'audit, de façon à les sensibiliser davantage au contenu.

2.g.13 Les travailleurs et leurs représentants devraient eux aussi participer aux programmes et au processus d'audit.

2.g.14 L'industrie devrait considérer la création d'un système pour améliorer l'échange d'information et d'expérience entre les diverses installations à l'intérieur d'une entreprise, ainsi qu'entre les entreprises, de façon à améliorer la compétence des vérificateurs. Cela pourrait, le cas échéant, comprendre l'échange d'équipes d'audit (ou des visites conjointes), ou encore le partage de rapports d'audits.

\section{h. MISE HORS SERVICE, FERMETURE ET DÉMOLITION}

2.h.1 Les procédures et les structures organisationnelles appropriées devraient être établies pour la mise hors service, la fermeture et la démolition des installations dangereuses.

" Ces procédures devraient être conçues pour faire en sorte que les dangers soient sous contrôle pendant le processus de fermeture et pendant que l'installation n'est pas en service, d'éviter de laisser un site contaminé une fois que l'installation a été fermée, de s'assurer que le processus de démolition se déroule de façon sûre, et enfin que le site est conforme à toutes les lois pertinentes concernant l'environnement et la sécurité.

" La direction devrait s'assurer que les contractants engagés pour la fermeture et la mise hors service respectent les consignes de sécurité.

\section{i. AUTRES RESPONSABILITÉS INDUSTRIELLES}

\section{Gérance des produits et aide aux autres entreprises}

2.i.1 Les producteurs de substances dangereuses devraient promouvoir la gestion sécuritaire des substances qu'ils produisent tout au long du cycle de vie de celles-ci, à partir de leur conception, en passant par leur production et leur utilisation, jusqu'à leur élimination finale, et ce conformément au principe de la "gérance des produits». Les producteurs devraient s'employer tout particulièrement à empêcher les accidents pendant la manipulation et l'emploi d'une substance dangereuse par les utilisateurs en aval.

" Les producteurs de substances dangereuses sont responsables de leurs produits et, par conséquent, devraient pleinement sensibiliser les personnes concernées aux dangers pouvant découler de l'utilisation, de la manipulation, de l'entreposage ou de l'élimination de leurs produits; ils devraient aussi, au besoin, fournir aide et (ou) conseils. 
" À cet égard, les producteurs devraient fournir la technologie, l'information et l'aide nécessaire aux contractants, distributeurs, transporteurs, clients et utilisateurs, de façon à leur permettre de suivre les pratiques de prévention appropriées. Les producteurs devraient être encouragés à fournir de leur plein gré aux clients la formation, les renseignements et d'autres services concernant les risques que présentent les produits chimiques et leur manipulation sans danger.

" Les producteurs devraient veiller à ce qu'une fiche signalétique santé et sécurité (FSSS) complète soit préparée pour chaque substance et mise à la disposition de tous les clients, dans la langue appropriée.

2.i.2 Les entreprises qui vendent des substances dangereuses devraient chercher activement à déterminer si leurs clients peuvent manipuler en toute sécurité les substances (y compris, s'il y a lieu, le traitement, l'utilisation et l'élimination des substances).

" S'il n'est pas possible de le déterminer, on devrait juger du bien-fondé d'accepter ce type de clients.

" Si on constate que des clients n'ont pas la capacité de manipuler en toute sécurité les substances dangereuses, le vendeur de celles-ci devrait prendre les mesures qui s'imposent (par exemple en aidant le client à acquérir cette capacité) ou alors ne pas accepter ce type de client.

"Les fournisseurs et les distributeurs de substances dangereuses devraient constituer des canaux d'information de premier plan à la disposition de certaines entreprises, notamment des PME, qui pourraient avoir besoin d'information et d'aide.

2.i.3 Les entreprises devraient chercher à coopérer avec d'autres secteurs dans leur région ou à l'intérieur de leur propre secteur, ou encore à établir des partenariats pour faciliter le partage de l'information et pour apprendre à partir de l'expérience.

2.i.4 Les associations industrielles/commerciales, les chambres de commerce locales et d'autres organisations industrielles et professionnelles devraient fournir des moyens utiles pour diffuser l'information concernant la prévention des accidents qui mettent en cause des substances dangereuses.

"Les associations industrielles/commerciales et les organisations professionnelles / normatives devraient être des sources critiques en matière d'orientation, de services de consultation et d'autres outils techniques, constituant ainsi un mécanisme pour canaliser l'expérience collective de leurs membres aux fins du développement des ressources qui peuvent être mises à la disposition aussi bien des membres que des non-membres.

"Les entreprises et les associations industrielles/commerciales devraient fortement encourager les entreprises qui agissent de façon moins responsable à améliorer et à respecter les objectifs appropriés en matière de sécurité.

2.i.5 Les entreprises et (ou) les associations industrielles/commerciales devraient encourager et aider les petites et moyennes entreprises et d'autres compagnies qui ont besoin d'assistance, en partageant leur expérience et en procurant des conseils et de l'aide aux fournisseurs, clients, contractants et à d'autres secteurs avec lesquels ils ont des 
interactions et (ou) ou des relations commerciales. À cet égard, les entreprises plus grandes devraient, par exemple :

" établir le dialogue avec les PME et d'autres compagnies grâce à des activités régionales, ou communiquer indirectement via les associations industrielles/commerciales et les organisations professionnelles;

" agir comme « guides » pour les PME, en s'alignant sur les principes de la gérance des produits, adoptés par beaucoup d'associations chimiques industrielles;

" agir comme conseillers pour les PME locales (ce que peuvent également faire les PME plus expérimentées);

" travailler avec les contractants pour s'assurer qu'ils respectent les normes de sécurité chaque fois qu'ils travaillent avec des entreprises de grande taille et, de façon plus générale, pour améliorer la capacité des contractants à accomplir leurs tâches de façon sûre, ce qui aidera à établir des relations à long terme, mutuellement bénéfiques;

" fournir de l'information sur la sécurité chimique aux contractants, clients et fournisseurs appropriés (sans attendre qu'on leur en demande);

" travailler avec les pouvoirs publics pour aider à élaborer une réglementation judicieuse et applicable.

" participer aux initiatives lancées par l'industrie en matière de sécurité, comme le Programme de gestion responsable $\mathrm{MD}^{\mathrm{MD}}$ l'industrie chimique.

\section{(Voir encadré texte sur la gestion responsableMD ci-dessous)}

2.i.6 Les entreprises plus petites, dont les ressources sont limitées, devraient examiner si, en matière de sécurité, elles ont besoin de l'aide de consultants externes, de spécialistes, d'associations industrielles ou commerciales et (ou) des pouvoirs publics, ainsi que des fournisseurs. Les fournisseurs de substances dangereuses devraient se montrer coopératifs en s'assurant que des personnes sont disponibles pour fournir des conseils permettant d'atteindre un niveau de sécurité approprié.

\section{PROGRAMME DE GESTION RESPONSABLEMD DE L'INDUSTRIE CHIMIQUE}

La Gestion responsable $\mathrm{MD}$ est une initiative élaborée et adoptée par l'association de l'industrie chimique pour améliorer la performance des procédés et des produits de leurs compagnies membres au niveau de la santé, de la sécurité et de l'environnement, ainsi que le niveau d'engagement communautaire et la sensibilisation de l'industrie.

En vertu de la Gestion responsable ${ }^{\mathrm{MD}}$, les compagnies participantes sont tenues de s'employer en permanence à améliorer la gestion responsable du secteur des produits chimiques, et s'entendent notamment à :

- améliorer en permanence leur performance au niveau de la santé, de la sécurité et de l'environnement;

- $\quad$ être attentifs aux préoccupations de la population et à y donner suite;

- $\quad$ s'entraider pour atteindre une performance optimale;

- $\quad$ faire part de leurs objectifs et progrès au public.

Pour plus de renseignements sur la Gestion responsable ${ }^{\mathrm{MD}}$, on peut visiter le site 


\section{Transfert de technologie}

\section{(Voir paragraphes 16.c.7 - 14 et 16.c.23 - 41 sur le transfert de technologie à l'échelle internationale)}

2.i.7 Chaque fois qu'une entreprise transfère une technologie de procédé ou une autre technologie liée à la sécurité, la direction de cette entreprise devrait tout faire pour s'assurer que la technologie sera appliquée d'une façon qui permette d'obtenir un niveau de sécurité équivalent à celui atteint dans les installations du fournisseur utilisant la même technologie.

* Il incombe aux entreprises qui transfèrent un procédé ou une autre technologie liée à la sécurité, destinés aux installations dangereuses, de mettre au point la technologie et les procédures d'exploitation connexes pour que les installations puissent fonctionner avec un niveau de sécurité acceptable, en tenant compte du fait qu'une technologie de sécurité donnée n'est peut-être pas adéquate pour toutes les installations et que les pratiques de la direction et d'autres employés peuvent être fortement influencées par l'administration et les usages à l'échelle locale.

W Tous ces transferts de technologie devraient être accompagnés de l'information connexe sur la sécurité.

n Le fournisseur de technologie devrait aider le destinataire en matière de formation.

" Le fournisseur de technologie ne devrait pas transférer une technologie jugée inacceptable à ses propres sites ou rejetée par les autorités compétentes pour des raisons de sécurité.

2.i.8 La technologie ne devrait pas être transférée à moins que le fournisseur et le destinataire soient convaincus, après une recherche des données et un examen d'une évaluation appropriée des risques, que le destinataire de la technologie peut l'appliquer et l'utiliser de façon sûre, en tenant compte des conditions locales ainsi que de l'infrastructure légale et administrative requise pour son exploitation sans danger.

* La partie chargée de procéder à cette évaluation - qui peut varier en fonction des clauses du contrat - devrait avoir accès à toute l'information nécessaire et utiliser les techniques actuellement acceptées pour l'identification des dangers et l'évaluation des risques.

" La partie responsable de l'évaluation devrait mettre à contribution les autorités locales et les représentants de la collectivité, et elle devrait veiller à ce que les résultats de l'évaluation soient mis à la disposition des autorités locales.

2.i.9 Un contrat devrait régir le transfert de la technologie, et ce contrat devrait notamment définir et réglementer clairement le partage des responsabilités entre les parties chargées du contrôle effectif de l'exploitation, de la prévention des accidents, ainsi que de la préparation et de l'intervention en matière d'accident. 
"Le cas échéant, ce contrat devrait également prévoir des clauses pour la livraison d'une usine clés en main.

" Les sections du contrat associées aux domaines décrits ci-dessus devraient être accessibles, sur simple demande, aux pouvoirs publics compétents ainsi qu'aux employés et à leurs représentants.

2.i.10 Lorsqu'une installation dangereuse faisant l'objet d'un transfert de technologie a été construite selon les spécifications données et que sa capacité de fonctionnement sans danger (conformément aux procédures spécifiées) a été démontrée de façon satisfaisante par une série d'épreuves d'admissibilité, un document de livraison devrait être signé par toutes les parties concernées, incluant les contractants.

\section{Acquisitions et activités affiliées}

\section{(Voir paragraphes 16.c.15 - 41 sur les investissements internationaux)}

2.i.11 Avant d'acquérir une installation dangereuse existante ou à l'état de projet, ou d'investir dans une installation de ce type, une entreprise devrait procéder à une évaluation des risques afin de déterminer la nature et le niveau des dangers à cette installation. L'entreprise devrait également déterminer les conditions pour exploiter l'installation en conformité à ses propres normes.

" Le «vendeur » d'une installation existante devrait divulguer tous les problèmes de sécurité connus ou suspectés, associés à cette installation.

" $\mathrm{L}^{\prime}$ « acheteur » devrait s'assurer que la divulgation est complète et que les mesures requises ont été prises pour permettre d'exploiter l'installation en toute sécurité après la prise de possession.

2.i.12 Toutes les politiques et lignes directrices de l'entreprise concernant la sécurité - soit la prévention, la préparation et l'intervention en matière d'accidents - devraient être applicables aux acquisitions.

" Lorsqu'une entreprise qui a acquis une installation existante en arrive, après une évaluation, à la conclusion que l'installation ne répond pas aux normes de l'entreprise ou n'atteint pas un niveau de sécurité accepté à l'échelle internationale, l'installation devrait être amenée à ce niveau dans un délai raisonnable.

"Dans les cas où la remise à niveau n'est pas possible, l'entreprise acquéreur devrait, en temps utile, informer les pouvoirs publics, les employés et leurs représentants de la situation et de ce qu'ils envisagent de faire.

2.i.13 Lorsqu'une entreprise a acquis partiellement une autre entreprise exploitant des installations dangereuses, mais sans aucun contrôle sur l'exploitation de cette dernière, l'acquéreur devrait, le cas échéant, négocier un accord contractuel pour aider à mettre en oeuvre durablement les normes de sécurité adéquates.

2.i.14 Une entreprise devrait donner à chacun de ses établissements affiliés ou de ses filiales pleinement accès à toute l'information concernant la sécurité - y compris l'information acquise récemment, les résultats de recherches, les éléments de technologie et de 
techniques de gestion qui pourraient réduire la probabilité d'accident ou atténuer les conséquences en cas d'accident - au site de l'affilié ou de la filiale.

2.i.15 Les entreprises devraient tenir des dossiers indiquant quelles substances dangereuses sont produites, utilisées ou entreposées chez les affiliés ou les filiales, en précisant l'emplacement géographique, de telle façon qu'il soit possible de partager effectivement à l'échelle mondiale l'information sur le potentiel d'accidents mettant en cause ces substances dangereuses.

2.i.16 Une entreprise devrait régulièrement procéder à un audit de la performance en matière de sécurité ainsi que des systèmes d'intervention en cas d'urgence, de toutes les installations dangereuses des filiales et, dans la mesure du possible, des affiliés, de façon à s'assurer que le niveau de sécurité à ces installations ne met pas indûment en danger les employés, les communautés voisines, l'environnement ou les biens, et que ce niveau soit conforme aux normes de sécurité généralement acceptées.

2.i.17 Lorsqu'elles déterminent le niveau de financement à accorder à une entreprise pour l'investissement dans une installation dangereuse, les institutions financières devraient tenir compte du montant de ressources nécessaires à l'entreprise pour se conformer aux exigences en matière de sécurité ainsi qu'aux politiques et lignes directrices sur la sécurité en vigueur dans l'industrie.

- 2.i.18 Dans le cas d'un accident, la direction d'une entreprise devrait en informer le plus tôt possible les gestionnaires des affiliés et des filiales concernés, en leur indiquant les causes probables et en leur recommandant de procéder immédiatement à des vérifications de la sécurité. De plus, tout rapport produit après l'accident (par exemple un rapport d'enquête) devrait être fourni aux gestionnaires des affiliés ou des filiales. 


\section{GLOBAL REPORTING INITIATIVE (GRI)}

Les rapports sur la performance des opérations industrielles sont importants pour les autorités et la population dans le cadre de la surveillance de la sécurité et de la situation environnementale propres aux compagnies. Pour éviter les nombreux rapports séparés, on préfère de plus en plus présenter plusieurs questions sous forme de rapport unique. On a commencé à utiliser des critères normalisés pour la production des rapports, de façon à pouvoir mieux comparer l'information et à faciliter le contrôle de qualité.

La GRI a été conçue comme un projet international multipartite pour l'élaboration, la promotion et la diffusion de structures pour la production volontaire de rapports sur la performance économique, environnementale et sociale d'une organisation (parfois appelés "rapports de durabilité »). La GRI veut rendre la production des rapports de durabilité aussi banale et crédible que les rapports financiers au niveau de la comparabilité, de la rigueur et de la vérifiabilité. Chaque secteur industriel peut adapter et élaborer davantage le cadre général mis au point par la GRI. La performance en matière de sécurité et les rejets accidentels, de même que les déchets et les émissions ainsi que les droits de la personne et les aspects du travail font partie du cadre général de ces rapports.

Un cadre généralement accepté pour les rapports de durabilité sera utile à tous les intervenants. La normalisation de la procédure encourage davantage de compagnies à produire des rapports pour le public en général. Les lignes directrices de la GRI permettent une meilleure comparabilité entre les diverses opérations, et font en sorte que tous les paramètres pertinents sont inclus dans les mesures et les audits. Cela est particulièrement important dans un domaine comme la santé et la sécurité où la production de rapports a évolué dans diverses directions, rendant les comparaisons difficiles. La production systématique de rapports permet la comparaison entre différentes compagnies et entre divers échéanciers, et peut montrer quand et où les initiatives lancées dans le domaine de la prévention, de la préparation et de l'intervention en matière d'accidents sont efficaces et concluantes.

Il est important également que les rapports produits dans le cadre de la GRI permettent aux profanes et aux organismes d'inspection de comprendre l'engagement d'une compagnie vis-à-vis entre autres, des politiques concernant l'environnement et la sécurité. Les gouvernements utiliseront l'information sur la durabilité pour localiser et surveiller les secteurs où des améliorations sont apportées par une compagnie, et négocier dans ces conditions les ententes sur les permis. Les agences de cotation et les investisseurs disposeront de l'information voulue pour la référenciation de la durabilité et les analyses des meilleures pratiques.

L'expansion rapide de l'utilisation du cadre de la GRI a des incidences importantes sur la façon dont les Principes directeurs sont appliqués, et sur la manière dont leur application peut être suivie.

On peut obtenir des renseignements supplémentaires à l'adresse suivante : www.globalreporting.org 


\section{Chapitre 3}

\section{POUVOIRS PUBLICS}

Le présent chapitre examine les rôles et les responsabilités des pouvoirs publics en matière de prévention des accidents mettant en cause des substances dangereuses. Il examine plus précisément la mise en oeuvre d'objectifs de sécurité et d'un cadre de contrôle, ainsi que de processus de surveillance et d'application de la réglementation. Le rôle des pouvoirs publics eu égard à d'autres aspects des processus de préparation, d'atténuation, d'intervention et de suivi (incluant, p.ex., la communication d'information au public et l'aménagement du territoire) sera examiné ailleurs dans la présente publication.

Il est important de se rappeler que l'expression "pouvoirs publics", telle qu'utilisée dans la présente publication, comprend toute la gamme d'organismes gouvernementaux à l'échelle locale, régionale et nationale, qui sont responsables de la protection de l'environnement, de la santé publique, de la santé et sécurité professionnelles, de la protection civile, de l'intervention en cas d'urgence et d'autres aspects de la prévention, de la préparation et de l'intervention en matière d'accidents chimiques. Le présent document ne précise pas quel type d'organisme gouvernemental devrait être responsable de telle ou telle activité, puisque chaque pays attribue diversement les responsabilités en se basant sur la réglementation et la culture locales.

\section{a. STRATÉGIE EN MATIÈRE DE SÉCURITÉ ET CADRE DE CONTRÔLE}

3.a.1 Les pouvoirs publics devraient mettre en oeuvre une stratégie à long terme pour réduire les risques d'accident mettant en cause des substances dangereuses, et pour limiter les conséquences néfastes d'accidents qui se sont produits. Cette stratégie devrait comprendre des objectifs clairs et appropriés.

3.a.2 Les pouvoirs publics devraient élaborer un cadre de contrôle clair, cohérent et méthodique, couvrant tous les aspects de la prévention des accidents qui mettent en cause des substances dangereuses, et limitant les conséquences de ces accidents.

" Le cadre de contrôle devrait être constitué d'obligations exécutoires (établies, par exemple, par des lois et des règlements). De plus, les pouvoirs publics devraient s'assurer que les normes, codes et règles d'orientation sont prêts (notamment les codes de pratique et les guides d'assurance de la qualité). Ces documents devraient être conçus de façon à permettre à chaque partie intéressée de déterminer si les objectifs de sécurité appropriés sont atteints.

" Le cadre de contrôle devrait également inclure des dispositions permettant de surveiller le niveau de sécurité des installations dangereuses pendant toutes les phases de leur cycle de vie.

" Les pouvoirs publics devraient s'employer à harmoniser le plus possible la réglementation au sein des diverses autorités nationales et locales, et à éliminer les exigences redondantes.

3.a.3 Les pouvoirs publics devraient promouvoir la coordination entre les divers organismes. Pour optimiser le processus de prévention, de préparation et d'intervention ainsi que l'utilisation des ressources, il est important que tous les organismes concernés coordonnent leurs activités. 
" Le processus de prévention, de préparation et d'intervention en matière d'accidents chimiques est par nature une activité interdisciplinaire dans laquelle sont engagées les autorités dans différents secteurs et à différents niveaux.

" Lorsqu'il y a plus d'un organisme public compétent, un mécanisme de coordination devrait être mis en place afin de réduire au minimum les activités redondantes et les conflits dans l'application des conditions imposées par les divers pouvoirs publics.

- Les pouvoirs publics devraient rechercher une certaine coordination entre eux pour faire en sorte que les règlements ainsi que les lignes directrices et l'information technique fournies aux entreprises soient complémentaires, non redondants et non contradictoires.

- 3.a.4 Lors de l'établissement des objectifs de sécurité et du cadre de contrôle, les pouvoirs publics devraient consulter les représentants d'autres intervenants concernés.

4Parmi ces intervenants, il pourrait y avoir les suivants :

- autres pouvoirs publics, y compris, le cas échéant, les représentants de communautés ou de pays voisins;

- secteur industriel (gestion et autres employés);

- associations professionnelles et industrielles/commerciales;

- experts indépendants;

- syndicats;

- groupes de pression;

- public.

" On devrait s'employer tout particulièrement à fournir à la population des occasions de contribuer à la prise de décisions par les pouvoirs publics.

3.a.5 Les objectifs et les exigences établis par les pouvoirs publics devraient être appliqués de façon équitable et homogène pour que les entreprises de toute taille et de tout type, aussi bien nationales qu'étrangères, soient obligées de respecter les mêmes objectifs globaux. Les pouvoirs publics devraient garantir un traitement égal à l'ensemble de l'industrie, quelle que soit la taille de l'installation, en offrant des chances égales à tous (même si le mode d'application peut varier). On devrait veiller à ce que les règlements et les programmes gouvernementaux ne soient pas biaisés en faveur des organisations plus importantes.

3.a.6 Le cadre de contrôle devrait donner une certaine souplesse aux méthodes utilisées pour respecter les objectifs et exigences en matière de sécurité. Le secteur industriel devrait, le cas échéant, être autorisé à mettre sur pied des méthodes qui sont mieux adaptées à sa nature propre pour lui permettre d'atteindre ses objectifs et respecter ses obligations.

" Les pouvoirs publics devraient considérer la répartition des exigences proportionnellement au niveau de risque.

" Un dialogue permanent entre les pouvoirs publics et l'industrie est nécessaire pour s'assurer que les règlements sont bien appliqués, ce qui permettra d'améliorer la sécurité, particulièrement en ce qui concerne les PME. 
3.a.7 Le cadre de contrôle devrait inclure des clauses pour l'application des conditions à observer, et suffisamment de ressources devraient être mises à la disposition des pouvoirs publics pour les activités de surveillance et d'application de la loi. Les mécanismes d'application devraient comprendre les sanctions appropriées et des amendes imposables en cas de non-conformité.

3.a.8 Les pouvoirs publics devraient donner des lignes directrices, claires et compréhensibles, sur la façon dont les objectifs peuvent être atteints par les entreprises dans le respect des conditions établies.

3.a.9 Les conditions et les lignes directrices établies par les pouvoirs publics devraient stimuler l'innovation et promouvoir l'emploi d'une technologie et de pratiques de sécurité améliorées.

" Les conditions à observer devraient être considérées comme minimales; on devrait encourager l'industrie à atteindre un niveau de sécurité plus élevé que celui qui correspondrait à une observation se limitant strictement aux normes et lignes directrices établies.

n Les pouvoirs publics devraient encourager l'industrie à prendre des mesures pour améliorer la sécurité, par exemple en appliquant les principes de la technologie intrinsèquement plus sûre.

(Voir paragraphes 2.c.6 - 8 sur la technologie intrinsèquement plus sûre)

3.a.10 Les pouvoirs publics devraient être conscients du fait que leurs décisions ou actions, même si elles ne mettent pas directement en cause une installation dangereuse donnée, peuvent contribuer à augmenter le risque d'accident ou d'effets nuisibles en cas d'accident. Par exemple, les pouvoirs publics devraient tenir compte du fait que les décisions afférentes à l'aménagement du territoire, à la planification pour les urgences, à l'intervention en cas d'urgence ou à la lutte antipollution peuvent influer sur la possibilité d'accidents ou encore aggraver les effets d'accidents.

3.a.11 Les pouvoirs publics devraient établir des critères pour déterminer si telle ou telle installation dangereuse peut être à l'origine d'accidents. Ces critères peuvent être basés, par exemple, sur des substances spécifiques et (ou) des catégories de substances présentes dans l'installation et sur les conditions de leur traitement, ainsi que sur leur potentiel d'exercer des effets nuisibles graves sur la santé, l'environnement ou les biens ${ }^{10}$.

3.a.12 Les pouvoirs publics devraient mettre sur pied un système pour obtenir de l'information concernant certaines catégories spécifiques d'installations dangereuses, en fonction de critères établis (voir paragraphe 3.a.11 ci-dessus). Dans le cadre d'un système de ce type, la direction des installations concernées serait tenue de présenter un rapport décrivant les dangers les plus importants à l'installation et de démontrer que les étapes appropriées ont été prises pour prévenir les accidents et atténuer les effets si un accident devait se produire ${ }^{11}$.

- Les pouvoirs publics peuvent établir différentes conditions de déclaration (rapports) pour différentes catégories d'installations, les plus contraignantes s'appliquant aux installations considérées comme présentant le plus grand potentiel de risque. 
" Le niveau de détail de ces rapports devrait être proportionné au niveau de danger propre à l'installation.

" Les rapports devraient se présenter sous un format harmonisé et utiliser des définitions uniformisées.

4 Les pouvoirs publics devraient examiner les rapports qu'ils reçoivent en déterminant, par exemple, s'ils sont complets, en évaluant la sécurité de l'installation visée et, le cas échéant, en procédant à des inspections sur place pour vérifier l'information figurant dans le rapport.

" Ces rapports devraient être accessibles au public, tout en se situant dans des limites raisonnables pour protéger la confidentialité (p. ex. les secrets commerciaux ${ }^{12}$, la vie privée) ou la sécurité publique, ou encore pour des raisons de défense nationale. Les rapports peuvent être obtenus sous forme de copie papier ou de fichier électronique. Le cas échéant, un résumé du rapport devrait être préparé et mis à la disposition du public.

\section{(Voir paragraphes 2.a16 - 18 sur les rapports de l'industrie en matière de sécurité)}

3.a.13 Les pouvoirs publics devraient veiller tout particulièrement à ce que toutes les installations dangereuses, y compris les PME et les utilisateurs commerciaux de substances dangereuses, entreprennent les évaluations appropriées de toute la gamme d'accidents possibles (incluant les accidents peu probables et ceux qui peuvent avoir des conséquences graves) ainsi que de planification adéquate pour les situations d'urgence.

3.a.14 Les pouvoirs publics devraient considérer quelles installations ou modifications d'installations peuvent être si dangereuses qu'elles ne devraient être autorisées à fonctionner qu'après autorisation réitérée par un pouvoir public déterminé. Dans ces cas, une certaine forme de contrat d'autorisation pourrait être adoptée, laquelle exigerait que la direction fournisse l'information détaillée sur tous les aspects pertinents des activités projetées par l'installation avant l'implantation et le démarrage et périodiquement par la suite. Le public devrait avoir la possibilité de contribuer aux décisions concernant ces contrats.

3.a.15 Les pouvoirs publics devraient limiter les formalités administratives/obligations de déclaration et se concentrer sur les éléments qui sont utiles pour identifier les risques et les moyens d'y faire face, ou qui sont nécessaires dans le cadre des fonctions gouvernementales. On sait que les besoins en formalités administratives pourraient représenter une charge importante pour les PME; cependant, la préparation des rapports et des plans pour les situations d'urgence constitue un important exercice d'apprentissage et représente une importante ressource en cas d'urgence. De plus, les pouvoirs publics ont légitimement besoin d'une information uniforme en provenance de toutes les installations dangereuses.

3.a.16 La réglementation et les notes d'orientation doivent être réexaminées régulièrement pour s'assurer qu'elles sont conformes aux objectifs suivants : réduire au minimum les risques; ne pas empêcher les améliorations au niveau de la conception; tenir compte des changements dans la technologie et de l'expérience acquise.

" Les conditions à observer et les notes d'orientation devraient, si nécessaire, être modifiées en temps opportun pour tenir compte des progrès techniques, des 
connaissances additionnelles acquises et des développements à l'échelle internationale.

D Pour les modifications au cadre de contrôle, qui exigent des changements dans la technologie ou dans les pratiques de gestion, il faudrait donner à l'industrie un délai raisonnable pour la mise en oeuvre et le respect de la conformité.

- 3.a.17 Les pouvoirs publics devraient promouvoir la sécurité en facilitant le partage de l'information concernant les systèmes de gestion de la sécurité et l'évaluation de ces systèmes et ainsi déterminer si d'autres travaux sont nécessaires.

\section{(Voir paragraphes 2.a.14 - 15 sur les systèmes de gestion de la sécurité)}

3.a.18 Les pouvoirs publics devraient disposer d'effectifs suffisants en personnel qualifié et formé, disponible pour assumer leur rôle et leurs responsabilités aux fins de la prévention des accidents, et ils devraient faire en sorte pour que ce personnel reçoive une formation théorique et pratique adéquate.

" Si l'expertise nécessaire aux pouvoirs publics pour assumer leur rôle et leurs responsabilités n'est pas disponible chez leur personnel, des dispositions devraient être prises pour que cette expertise soit obtenue, si nécessaire, en retenant, par exemple, les services de consultants externes.

- Les contrats d'experts ou de consultants externes engagés par les pouvoirs publics devraient stipuler que ces experts ou consultants ne doivent divulguer aucune information non publique recueillie, sauf à l'organisme public qui a retenu leurs services.

3.a.19 Les pouvoirs publics devraient exiger que la direction d'une installation dangereuse déclare certains incidents (accidents et quasi-accidents).

" L'information pertinente contenue dans ces rapports devrait être facilement accessible pour éviter des incidents similaires à d'autres installations dangereuses.

Les pouvoirs publics devraient également mettre sur pied un système pour conserver les statistiques sur les accidents, effectuer des analyses de l'information recueillie, et enfin diffuser les renseignements pertinents obtenus grâce à ces analyses.

3.a.20 Pour aider l'industrie à améliorer la sécurité aux installations dangereuses, les pouvoirs publics devraient considérer le déploiement d'autres activités, comme les suivantes: fournir de l'aide technique; promouvoir les programmes de formation; encourager la recherche; faciliter la sensibilisation du public. Ces activités devraient être conduites de façon à ne pas nuire au jugement impartial des pouvoirs publics dans leur rôle premier, soit d'établir et de faire respecter les objectifs et conditions à observer en matière de sécurité.

3.a.21 Les pouvoirs publics devraient promouvoir les activités d'aide (par eux-mêmes, l'industrie ou d'autres) pour améliorer les programmes de sécurité dans les installations dangereuses des PME et d'autres entreprises qui pourraient connaître des contraintes comparables (p.ex. en expertise, en personnel) pour la mise en oeuvre de ces programmes. Les mesures d'aide par les pouvoirs publics devraient être déployées 
séparément des programmes d'application réglementaire. Parmi les mesures spécifiques que les pouvoirs publics peuvent prendre, on peut citer les suivantes:

" Prévoir des mécanismes pour permettre aux PME de faire entendre leur point de vue sur la réglementation et proposer d'autres approches, comme le protecteur du citoyen, les comités consultatifs et les forums locaux d'échange d'information.

" Prévoir un accès en une seule étape à l'information pertinente et à l'aide technique, p. ex. via des centres d'information et des lignes téléphoniques sans frais.

" Donner des lignes directrices et fournir des outils techniques, comme des programmes d'audit certifiés.

4 Promouvoir les services de volontariat par les entreprises (p. ex. pour aider d'autres entreprises dans leur industrie ou communauté qui pourraient avoir besoin d'assistance, notamment certaines PME).

" Fournir des renseignements ainsi que la formation théorique et pratique, incluant l'expérience acquise, les bases de données et les communications techniques.

" Pour l'amélioration de la sécurité, promouvoir les activités volontaires, qui vont audelà des exigences réglementaires.

\section{(Voir paragraphe 1.19 et encadré texte sur les PME)}

\section{b. AMÉNAGEMENT DU TERRITOIRE}

L'élaboration et la mise en oeuvre des clauses d'aménagement du territoire (aussi bien le zonage que l'implantation) contribuent à la fois à prévenir et à atténuer les accidents mettant en cause des substances dangereuses. L'aménagement du territoire peut être considéré comme une mesure préventive, du fait qu'il peut aider à implanter l'installation dangereuse à une distance adéquate des autres installations ou aménagements, ce qui permettrait de prévenir les effets nocifs; ou encore, il peut être considéré comme un moyen pour réduire les effets nuisibles des rejets, des incendies, des explosions ou d'autres accidents qui ont lieu.

$V u$ le double rôle de l'aménagement du territoire, on en parle également dans le chapitre 6. Pour avoir une idée complète du sujet, il est important de lire les deux ensembles de dispositions.

Bien que l'aménagement du territoire soit un élément essentiel dans la stratégie de réduction des risques liés aux accidents avec présence de substances dangereuses, il est complémentaire aux autres mesures de prévention et d'atténuation, mais ne peut les remplacer.

Il est important de comprendre que l'aménagement du territoire dans ce contexte s'applique non seulement au zonage et à l'implantation des installations dangereuses, mais également aux modifications importantes des installations existantes. Il est indispensable aussi de tenir compte des divers aspects de l'aménagement du territoire lors du processus décisionnel concernant les projets d'aménagement au voisinage d'une installation dangereuse existante (notamment les foyers pour personnes âgées, les écoles, les magasins et d'autres propriétés commerciales, ainsi que les infrastructures publiques, comme les gares de chemin de fer).

À noter que dans certains pays l'aménagement du territoire est décidé à l'échelle nationale en coopération avec les autorités locales, alors que dans d'autres il s'agit d'une question exclusivement locale. 
3.b.1 Les pouvoirs publics devraient élaborer des dispositions pour l'aménagement du territoire en s'assurant que les nouvelles installations dangereuses soient implantées de façon à protéger la santé, l'environnement et les biens en cas d'accident mettant en cause des substances dangereuses.

" Ces dispositions devraient également prévenir l'aménagement d'habitations ou d'autres développements inappropriés près d'installations dangereuses, et exercer un contrôle sur les modifications inopportunes aux installations existantes.

" L'aménagement du territoire devrait examiner les deux éléments suivants :

- le zonage général, qui comprend la création de zones spécifiques pour les activités industrielles dangereuses en tenant compte de tous les aspects de protection de la santé, de l'environnement et des biens;

- le processus décisionnel au cas par cas, concernant l'implantation d'une nouvelle installation, les modifications importantes à une installation existante, ou des travaux d'aménagement près d'une installation existante.

מ Dans de nombreuses régions, les installations dangereuses sont situées près de secteurs habités ou commerciaux, ou encore près de milieux sensibles. Ces dernières ne seraient pas conformes aux normes d'aménagement du territoire pour les nouvelles installations ou d'autres aménagements ( $\mathrm{du}$ fait, par exemple, que les normes ont changé avec le temps ou qu'elles n’ont pas été appliquées avec rigueur dans le passé). Les pouvoirs publics devraient, si possible, faire en sorte que les aménagements existants se conforment aux normes actuelles d'aménagement du territoire.

\section{(Voir paragraphe 2.c.1-3 traitant de l'implantation des installations)}

3.b.2 Les pouvoirs publics devraient, lorsqu'ils envisagent un projet de nouvelle installation dangereuse ou un projet de développement près $d$ 'une installation existante, tenir compte des risques d'accident potentiel (y compris les effets nuisibles en cas d'accident). L'évaluation du risque devrait être effectuée par les pouvoirs publics ou en leur nom; la direction de l'installation dangereuse visée devrait mettre l'information requise à la disposition des pouvoirs publics.

n L'évaluation du risque devrait tenir compte de toute la gamme d'incidences, ainsi que des avantages et des inconvénients de l'emplacement particulier proposé pour la nouvelle installation ou le nouvel aménagement. Cela devrait être fait pour les projets de nouvelles installations, les modifications importantes apportées à des installations existantes, et pour d'autres lotissements au voisinage d'installations dangereuses.

D Les autorités responsables de l'aménagement du territoire devraient obtenir l'information technique concernant le risque présenté par l'installation dangereuse considérée (figurant, par exemple, dans des notifications adressées aux pouvoirs publics ou dans des rapports sur la sécurité), et en tenir compte. Cette information devrait être disponible auprès de l'entreprise concernée. Les responsables de l'aménagement devraient également tenir compte de toute autre information accessible, comme les rapports provenant d'universités ou d'ONG.

- Une méthode systématique d'identification des dangers, d'estimation et d'évaluation des risques est utile pour fournir aux pouvoirs publics les lignes directrices qui les 
aideront à prendre leurs décisions en matière d'aménagement du territoire. Par exemple, une méthode systématique pourrait conduire à un classement relatif des dangers et des risques.

" Lors des prises de décision concernant l'aménagement du territoire, les évaluations du risque sont une source d'information pour le processus décisionnel, mais elles ne constituent pas toujours la seule influence décisive. Ces décisions sont également tributaires du jugement socio-politique au niveau local. À cet égard, les pouvoirs publics devraient expliciter tous les critères retenus pour guider les décisions concernant l'aménagement du territoire, y compris les critères pour l'analyse de la tolérabilité/acceptabilité des risques et pour le processus décisionnel; les conclusions devraient être transparentes.

\section{(Voir section $2 . b$ traitant de l'identification des dangers et de l'évaluation des risques)}

3.b.3 Les décisions par les pouvoirs publics en matière d'aménagement du territoire, concernant les installations dangereuses, devraient tenir compte du risque cumulatif représenté pour la communauté par toutes les installations dangereuses situées à proximité. Dans certains cas, il pourrait être préférable, du point de vue de la sécurité, de centraliser toutes les installations dangereuses en un seul endroit, alors que dans d'autres, il vaudrait peut-être mieux les garder éloignées les unes des autres.

4Dans les décisions concernant l'aménagement du territoire, on devrait tenir compte de la possibilité d'effets domino, où un accident chimique à un site pourrait entraîner un accident à un (des) site(s) voisin(s).

" Dans les décisions, on devrait considérer l'importance de maintenir une distance adéquate entre une installation dangereuse et d'autres secteurs aménagés situés à proximité, de façon à réduire les risques d'effets nuisibles en cas d'accident.

"Dans les décisions, on devrait considérer l'importance de prévoir une distance adéquate entre une installation dangereuse et la population ou les milieux sensibles de façon à réduire les risques d'effets nuisibles en cas d'accident.

3.b.4 Les rôles des pouvoirs publics eu égard aussi bien à l'établissement d'objectifs pour la sécurité qu’à l'aménagement du territoire devraient être bien coordonnés et bénéficier d'une communication interne efficace.

\section{c. EXAMEN ET ÉVALUATION DE LA PERFORMANCE EN MATIÈRE DE SÉCURITÉ}

\section{(Voir également la sect. 2.g sur l'examen et l'évaluation de la performance en matière de gestion de la sécurité)}

3.c.1 Les pouvoirs publics devraient établir des programmes appropriés d'examen et d'évaluation de la performance en matière de sécurité (incluant des inspections) pour le suivi de celle-ci aux installations dangereuses à toutes les phases de leur cycle de vie. Cela comprend la planification, l'implantation, la conception, la construction, l'exploitation (incluant la maintenance), ainsi que la mise hors service / fermeture / démolition.

" Les inspections sont essentielles lorsqu'on veut s'assurer de la sécurité globale d'une installation dangereuse, car elles permettent de vérifier si les règlements, les normes 
et les pratiques sont bien observés, si les systèmes de gestion de la sécurité sont en place et fonctionnent correctement, et enfin si les rapports sur la sécurité sont valides. Elles constituent également un moyen pour apprendre comment améliorer les systèmes de gestion de la sécurité, et elles peuvent stimuler l'industrie en l'incitant à aller au-delà des conditions minimales. Un autre avantage important des inspections est qu'elles constituent le fondement de la confiance que la population place dans la sécurité des installations dangereuses.

" Les autorités chargées de l'inspection devraient jouer, selon le cas, différents rôles :

- le rôle traditionnel de l'inspecteur - vérifier la conformité à toutes les exigences - s'est élargi, l'inspecteur jouant maintenant un deuxième rôle important, soit $\mathrm{d}$ 'aider les entreprises à détecter les problèmes potentiels (même si elles ne sont pas soumises à des exigences réglementaires) et à fournir de l'information sur les moyens d'améliorer la performance en matière de sécurité;

- les autorités chargées de l'inspection sont également engagées dans des activités connexes - conçues pour aider à atteindre l'objectif général d'amélioration de la sécurité -, comme l'élaboration de lignes directrices pour la préparation et la mise en oeuvre des programmes d'audit, la fourniture de services de consultation et la promotion de programmes volontaires d'auto-audit.

" Il est possible qu'une inspection ne suffise pas à examiner de façon détaillée tous les aspects concernant la sécurité à une installation dangereuse. L'inspection pourrait donc être axée sur l'évaluation du système de gestion et, plus précisément, sur l'évaluation de l'efficacité globale de ce système (autrement dit, l'inspection devrait déterminer si l'entreprise assume ses responsabilités en matière de sécurité).

" Dans certains cas, les inspections comprendront des examens plus détaillés. Cela peut se produire dans le cadre d'un plan d'inspection générale (p. ex. lorsque les autorités déterminent des secteurs prioritaires spécifiques à partir d'un plan stratégique) ou suite à la performance médiocre à une installation ou à d'autres problèmes qui ont été constatés (p. ex. lorsque l'inspection révèle des problèmes potentiels ou s'il y a des questions suite à l'examen du rapport sur la sécurité par les autorités).

"Les inspections constituent un élément important, mais elles représentent seulement une partie du système de contrôle par les pouvoirs publics. Il y a d'autres éléments, comme l'attribution de permis, la documentation et les examens.

3.c.2 Les pouvoirs publics devraient s'assurer que des lignes directrices sont élaborées à l'intention de ceux qui doivent se conformer et sur la façon dont ils peuvent assumer au mieux leurs obligations et le démontrer aux autorités chargées de la surveillance et de l'application des règlements.

3.c.3 Pour que la surveillance soit efficace et crédible, les autorités chargées de l'inspection et des activités de surveillance connexes devraient pouvoir en rendre compte publiquement. Cela peut être réalisé en rendant le système transparent.

" À cette fin, les autorités chargées des inspections et des activités de surveillance connexes devraient rendre publics les objectifs, les politiques et les procédures qu'elles ont adoptés pour les activités de surveillance. 
" Les pouvoirs publics devraient également rendre publics les résultats de leurs activités de surveillance.

" Cela peut aider à établir et à maintenir un climat de confiance entre les intervenants (pouvoirs publics, industrie, employés, population et autres).

" Les pouvoirs publics (et l'industrie) devraient se mobiliser pour mettre à la disposition du public l'information pertinente sous une forme qui peut facilement être comprise, et pour susciter des occasions de dialogues entre les intervenants (industrie, pouvoirs publics et population).

" Il faut également aider la population à mieux comprendre la nature du « risque » et les risques que représentent les installations dangereuses.

3.c.4 Tous les programmes d'inspection devraient comporter des éléments communs (qui sont essentiels si on veut qu'un programme d'audit ou d'inspection soit efficace). Chaque programme doit, plus précisément, comprendre les éléments suivants :

- objectifs clairement définis;

- portée délimitée : elle ne doit pas être trop large, car il pourrait alors être difficile d'atteindre l'objectif souhaité;

- calendrier (incluant p. ex. un plan d'action avec des échéanciers);

- $\quad$ expert(s) formé(s) et qualifié(s) pour les tâches et objectifs spécifiques;

- examen(s) de la documentation appropriée, et entrevues avec le personnel clé (incluant les opérateurs de procédés);

- détermination des carences et des pratiques adéquates;

- rapport officiel présentant les données recueillies;

- examen par la direction définissant clairement les responsabilités pour les mesures de suivi, et moyen pour s'assurer que les mesures sont appliquées;

- démonstration que les mesures de suivi ont été appliquées.

3.c.5 La participation des pouvoirs publics aux activités de surveillance, y compris les inspections, ne change rien au fait que la responsabilité première pour la sécurité des installations dangereuses incombe à la direction.

3.c.6 Les pouvoirs publics devraient partager à l'intérieur du pays et à l'échelle internationale l'information et l'expérience concernant les méthodologies et les outils utilisés pour les inspections, en plus d'échanger les résultats d'inspections spécifiques. On devrait s'employer à promouvoir en permanence ces activités et à mettre sur pied des mécanismes qui pourraient faciliter le partage de l'information.

" Pour partager l'expérience entre les inspecteurs, on devrait s'employer encore plus à promouvoir les « visites mutuelles conjointes » pour les inspections.

" De plus, des réseaux internationaux d'inspecteurs devraient être constitués pour divers aspects de la sécurité chimique.

" Une des façons d'améliorer l'apprentissage grâce à l'expérience des autres est de mieux harmoniser la réglementation et la cohérence des méthodes associées à la surveillance et aux inspections. 
" Les méthodes employées pour les inspections devraient tenir compte de la réglementation et des coutumes locales; par conséquent, il ne sera peut-être pas toujours possible d'atteindre une certaine uniformité dans les méthodes.

3.c.7 On devrait s'employer à utiliser des indicateurs de performance plus avancés comme moyen possible pour mesurer la sécurité et déterminer si les mesures prises permettent effectivement de réduire le risque. De plus, ces indicateurs pourraient aider à axer les audits et les inspections vers les secteurs de haute priorité13.

3.c.8 Les pouvoirs publics devraient établir des programmes pour une ou plusieurs inspections par année, pour l'établissement d'objectifs et de priorités - p. ex. se concentrer pendant une année sur un sujet particulier, comme les installations gérées par plusieurs exploitants -, ainsi que des échéanciers.

" Pour l'établissement des objectifs et des priorités, les autorités devraient tenir compte de la performance passée des installations dangereuses en matière de sécurité, ainsi que de la nature et de la portée des dangers présents dans les installations.

" Normalement, les programmes d'inspection comporteraient, selon le cas, des dispositions aussi bien pour les inspections planifiées que pour les «vérifications ponctuelles» (p.ex. lorsqu'il existe un secteur préoccupant). Les inspections planifiées et les vérifications ponctuelles ont toutes une fonction importante.

" La préparation longtemps à l'avance des plans d'inspection présente un avantage important, à savoir que cela donne aux autorités la possibilité de former et d'équiper leurs inspecteurs pour leur permettre de mener à bien leurs inspections.

3.c.9 En matière d'inspections, les pouvoirs publics devraient élaborer une méthode cohérente et normalisée pour la planification, l'exécution et la production de rapports.

" Toutes les inspections devraient comprendre la documentation sur les résultats, y compris les recommandations pour les mesures de relance et pour toute action coercitive requise.

" Les mesures de suivi devraient être conçues de telle façon que les déficiences détectées soient traitées de façon appropriée et en temps opportun, et qu'il y ait une vérification des mesures prises.

"Différents outils sont à la disposition des pouvoirs publics pour les mesures de suivi, selon la gravité des cas, comme des notifications des modifications à effectuer; la description des mesures et des échéanciers acceptés; des citations et amendes et enfin, dans les cas les plus graves, la fermeture de l'installation.

3.c.10 Les inspections devraient être faites par un inspecteur ou des inspecteurs aidé(s), si nécessaire, par des experts pour examiner les dangers particuliers notés à l'installation.

" Une tierce partie (indépendante du gouvernement et de l'entreprise) peut être déléguée pour procéder, au nom des pouvoirs publics, à des inspections techniques ou à des inspections des systèmes. On devrait s'assurer de la qualité de ces tierces parties (par exemple, par des processus de certification ou d'accréditation). 
" Même si une tierce partie est engagée dans le processus, les pouvoirs publics demeurent légalement responsables des inspections; ils ne peuvent pas déléguer leurs responsabilités aux inspecteurs de la tierce partie.

"Lorsqu'il y a participation d'une tierce partie, il faut prendre soin d'éviter tout conflit éventuel, notamment lorsque cette tierce partie est retenue à la fois pour des services de consultation et d'inspection.

3.c.11 Suffisamment de ressources et de personnel devraient être mises à la disposition des pouvoirs publics pour leur permettre d'effectuer les inspections. Les inspecteurs travaillant pour les pouvoirs publics devraient recevoir la formation appropriée et posséder l'expertise nécessaire pour déterminer, par exemple, si les méthodes adoptées dans une installation dangereuse permettront à celle-ci de se conformer aux exigences légales.

3.c.12 Les pouvoirs publics participant au processus de prévention, de préparation et $d^{\prime}$ intervention en matière d'accidents chimiques (p. ex. ceux qui sont responsables de la santé, de la sécurité, de l'environnement et de la protection civile, aux échelles nationale, régionale et locale) devraient coopérer et coordonner leurs actions dans le domaine des inspections. Cela aidera à maximiser l'efficacité, réduira au minimum le double emploi des ressources (lesquelles sont limitées) et contribuera effectivement à la gestion du risque. Une coopération de ce type devrait s'atteler, selon les besoins, aux diverses phases du processus d'inspection (p. ex. préparation, inspection sur place, rapport et suivi).

" La coopération apporte un certain nombre d'avantages connexes, notamment les suivants : apprendre mutuellement de l'expérience des autres; partager les ressources, l'expertise et les outils; tirer avantage de points de vue différents; réduire au minimum la probabilité que différents organismes donnent des conseils contradictoires ou en arrivent à des conclusions incompatibles; faciliter l'amélioration de la réglementation ou des normes, ainsi que la résolution des conflits (p. ex. entre sécurité et environnement); améliorer le climat de compréhension et de confiance entre les divers organismes. La coopération devrait donc mener à une plus grande cohérence au niveau des approches et des résultats entre les diverses équipes d'inspection à l'intérieur d'un même pays.

" L'industrie tire elle aussi des avantages de cette coopération. Par exemple, la coopération pourrait conduire à des inspections plus ciblées, permettre d'éviter le double emploi, et donner davantage de cohérence dans les approches par les divers organismes concernés. Il devrait en résulter moins d'interruptions dans les activités commerciales et une participation plus efficiente des employés aux inspections.

4 Une coordination efficiente des inspections entre les divers organismes concernés nécessite beaucoup d'efforts pour en arriver à une compréhension mutuelle des différentes coutumes, réglementations et structures de ces organismes.

"Dans le cadre des étapes nécessaires pour une coordination effective, les autorités devraient établir :

- s'il y a lieu, des procédures pour des inspections conjointes;

- un partage clairement défini des tâches, avec détermination des rôles et des responsabilités pour divers aspects des inspections; 
- un climat de compréhension mutuelle entre les autorités pour tous les aspects pertinents de la culture propre à ces autorités, incluant les outils légaux, les politiques et les procédures;

- des activités de formation coordonnées;

- des canaux de communication clairement déterminés;

- un mécanisme bien défini pour traiter les conflits.

" Une inspection coordonnée ne signifie pas que l'inspection est pleinement intégrée. L'objectif devrait plutôt viser à éviter le double emploi des activités et à partager les travaux correspondant à divers aspects des inspections lorsqu'il y a un intérêt commun.

3.c.13 Les inspecteurs (pouvoirs publics) et l'industrie réglementée devraient coopérer lorsqu'ils procèdent à des audits et à des inspections. Cette coopération peut prendre différentes formes, y compris une coordination améliorée des activités et de la communication dans des domaines d'intérêt commun, ainsi qu'un grand esprit d'ouverture lors des discussions au sujet des résultats des audits et des calendriers. De plus, la coopération peut également aider les autorités à tirer parti des résultats des audits effectués par l'entreprise ou une tierce partie.

" Un système de réglementation efficace est indispensable si on veut instaurer une bonne coopération et un esprit de confiance entre l'industrie et les pouvoirs publics. La réglementation est l'effet multiplicateur nécessaire pour que les pouvoirs publics puissent protéger les intérêts du public et des employés.

" Grâce à la coopération on peut généralement apporter certaines améliorations, dont les suivantes:

- augmentation de l'efficacité des inspections, ce qui permet aux pouvoirs publics, à l'industrie et à d'autres secteurs d'optimiser l'utilisation de ressources limitées (y compris le personnel);

- constitution d'une base permettant aux autorités de diminuer la fréquence ou de changer la nature des inspections en fonction de l'information qui leur est fournie;

- amélioration de la capacité d'apprentissage mutuel des parties, qui pourront ainsi mieux assumer leurs rôles et responsabilités (p. ex. le processus d'audit peut être amélioré grâce aux conseils des autorités chargées des inspections);

- augmentation du niveau de confiance et de l'engagement parmi les intervenants (y compris les employés et le public).

" lorsqu'ils s'engagent à coopérer avec l'industrie, les pouvoirs publics devraient s'assurer que cette coopération ne nuit pas à leur capacité d'appliquer les lois et qu'elle ne diminue pas leur indépendance.

" pour que la coopération soit efficace, la direction d'une installation dangereuse devrait être compétente et désireuse de s'attaquer sérieusement aux problèmes liés à la sécurité. De plus, elle devrait faire part des résultats des audits aux pouvoirs publics.

3.c.14 Les pouvoirs publics devraient considérer la coordination de divers aspects de la sécurité, de la santé et de l'environnement, si cela peut procurer des avantages évidents, et ce parallèlement aux efforts de l'industrie chimique et d'autres secteurs industriels 
visant à améliorer l'intégration de la gestion des questions de sécurité, de santé et d'environnement afin de pouvoir les traiter de façon plus efficace et plus efficiente. 


\section{Chapitre 4}

\section{PUBLIC ET AUTRES INTERVENANTS}

Le présent chapitre porte sur les rôles et responsabilités de divers intervenants, autres que l'industrie et les pouvoirs publics. On s'intéresse tout particulièrement aux collectivités et au public, aux syndicats, aux établissements de recherche universitaires ou autres, et enfin aux organisations non gouvernementales.

\section{a. COLLECTIVITÉS/PUBLIC}

\section{(Voir encadré texte après la sect. 4.a « Exemple de représentation de la communauté »)}

4.a.1 Les membres des communautés situées au voisinage d'installations dangereuses et d'autres qui peuvent être touchées en cas d'accident, devraient être au courant des risques d'accident, savoir où obtenir de l'information au sujet des installations et comment réagir en cas d'accident.

" Il est probable que la sensibilisation du public conduise à une réduction du nombre d'accidents chimiques et de leur gravité. Par exemple, le fait que le public est bien informé peut inciter l'industrie à réduire les risques d'accident chimique. De plus, un public bien informé peut stimuler le dialogue entre l'industrie, les pouvoirs publics et la population, et faciliter la participation effective de cette dernière au processus décisionnel concernant les installations dangereuses.

" Mieux le public sera informé des risques pour sa communauté, plus il voudra participer aux processus décisionnels et prendre des initiatives pour aider à réduire les risques.

4.a.2 Les collectivités situées à proximité d'installations dangereuses devraient, le cas échéant, avoir des représentants qui peuvent assurer la liaison entre celles-ci et les autres intervenants et faciliter l'échange d'information.

" Ces représentants pourraient, par exemple, être choisis par les administrations locales, ou encore se présenter, de leur propre initiative, comme volontaires.

" Les représentants des collectivités peuvent être considérés comme des partenaires des autres intervenants et aider à faire connaître les points de vue et les préoccupations des collectivités et à mettre en place des moyens pour permettre aux installations de travailler avec les membres des collectivités.

" L'existence des représentants des collectivités ne modifie ni ne limite aucunement les rôles et les responsabilités de l'industrie ou des pouvoirs publics.

4.a.3 Les représentants des collectivités peuvent aider les autres intervenants à informer et à former le public, et à faire part des réactions des collectivités aux installations et aux pouvoirs publics. 
" Les représentants des collectivités devraient rechercher activement l'information concernant les installations dangereuses. Ces représentants devraient également être connus des autorités des installations dangereuses et des pouvoirs publics, de façon à ce que l'information puisse leur être transmise.

" Les représentants des collectivités devraient coopérer avec les pouvoirs publics et, le cas échéant, avec les représentants des installations dangereuses afin d'aider à élaborer des programmes de communication et des messages. La participation des représentants des collectivités peut faciliter la diffusion de l'information sur les dangers, leur compréhension par les milieux ciblés, et leur réception.

" Les représentants des collectivités devraient, selon le cas, diffuser l'information qu'ils ont obtenue. Cela pourrait être réalisé par différents moyens, comme les journaux, Internet, des tableaux d'affichage, etc. 


\section{EXEMPLE DE REPRÉSENTATION DE LA COMMUNAUTÉ : COMMENT METTRE SUR PIED UN COMITÉ DE CITOYENS POUR LES QUESTIONS DE PRÉVENTION, DE PRÉPARATION ET D'INTERVENTION EN MATIËRE D'ACCIDENTS CHIMIQUES}

Une des façons de faire participer davantage les membres de la communauté et de faciliter les échanges entre les membres d'une communauté et d'autres intervenants est de créer un comité dont les membres représentent les divers intérêts de la communauté. Bien que la liste ne soit pas exhaustive, les éléments suivants mettent en lumière un certain nombre de questions dont il faut tenir compte si on veut former un comité fonctionnel et représentatif.

La qualité de membre du comité est importante, car ce dernier devrait refléter les intérêts de la communauté. Les membres devraient provenir de différents secteurs de la communauté, ainsi que de divers milieux. Comme c'est généralement le cas aux États-Unis et au Canada, le comité comprend des représentants de l'industrie locale, de l'administration municipale, des organisations non gouvernementales et des employés d'installations voisines, ainsi que des éducateurs, des personnes actives dans la communauté et de simples citoyens.

Pour faciliter le démarrage du comité, on pourrait faire appel aux services d'un consultant externe et neutre. L'installation dangereuse pourrait aider le processus en désignant des groupes cibles à l'intérieur de la communauté et en les invitant à participer.

Pour encourager la participation effective des citoyens à l'échelle locale, le comité pourrait attirer les personnes possédant des qualifications pertinentes. Une des façons d'y arriver est de faire appel aux retraités (p. ex. anciens juristes, ingénieurs, spécialistes en environnement, etc.)

Normalement, les membres de la communauté qui participent au comité le font volontairement. Il est donc important de faciliter leur participation (p. ex. en organisant les réunions à des dates, heures et endroits appropriés) et de trouver des moyens pour exprimer combien le travail des participants est apprécié. De plus, l'atmosphère devrait refléter l'existence d'une mission commune, être amicale et décontractée, bref un endroit où on peut apprendre à travailler ensemble. Cela facilitera la communication et aidera à créer un niveau élevé de confiance entre les intervenants.

Le comité devrait déterminer son mandat et ses objectifs (en consultant les intervenants appropriés) et définir ses propres activités pour atteindre ces objectifs. Cela devrait être fait en tenant compte des conditions locales et des capacités des membres du comité. On devrait considérer la possibilité de faire appel à un médiateur neutre (payé ou non) pour faciliter les réunions du comité.

La direction d'une installation dangereuse et les représentants des pouvoirs publics devraient traiter les membres du comité comme des partenaires. Un comportement paternaliste de la part des représentants d'entreprises locales ou des pouvoirs publics pourrait nuire aux relations et altérer les échanges entre les intervenants.

Pour assurer sa viabilité, le comité pourrait obtenir un financement. Cependant, pour préserver l'indépendance du comité, ce financement ne devrait couvrir que ses dépenses. Le financement pourrait provenir de diverses sources, comme de la direction de l'installation dangereuse, d'associations commerciales ou industrielles, ou encore des pouvoirs publics.

Un réseau pour l'échange d'information et pour la communication devrait être mis sur pied dans chaque comité. Enfin, on devrait trouver des moyens pour permettre aux différents comités de partager leur expérience. 


\section{b. ORGANISATIONS SYNDICALES}

- 4.b.1 Les organisations syndicales devraient encourager la formation théorique et pratique de leurs membres dans le domaine de la sécurité.

4 Cela pourrait se traduire par une formation directe, ou par une aide aux activités de formation grâce, par exemple, à l'élaboration de plans de cours, de la fourniture de matériels et de programmes de formation, d'engagement de tuteurs et de conférenciers, et enfin, d'assistance avec partage de l'expérience liée à la formation.

" La compétence et les connaissances acquises par les organisations syndicales grâce à leurs programmes de formation théorique et pratique et à leur expérience de tous les jours, peuvent servir à améliorer les politiques et les activités de prévention. Étant donné que les syndicats sont directement intéressés par la réussite de l'entreprise dans laquelle travaillent leurs membres, ils peuvent être des alliés très utiles pour la réalisation d'un haut niveau de sécurité.

" Les organisations syndicales aident également à former les représentants en sécurité. Ces derniers sont chargés de s'occuper des questions de santé et de sécurité de leurs collègues. Ils jouent également des rôles importants au niveau de la médiation et de la communication entre la direction et les autres employés.

\section{(Voir paragraphe 2.d.28 sur les représentants en sécurité)}

4.b.2 Les organisations syndicales devraient faciliter la coopération avec la direction à l'échelle tant nationale qu'internationale.

4.b.3 Les syndicats devraient participer aux organisations internationales qui élaborent des lignes directrices sur la sécurité chimique et la prévention des accidents. Par exemple, en tant que l'un des trois groupes constitutifs de l'OIT, les organisations syndicales jouent et continuent de jouer un rôle de premier plan dans l'élaboration et la promotion des conventions et des recommandations de l'OIT.

\section{c. ÉTABLISSEMENTS DE RECHERCHE UNIVERSITAIRES ET AUTRES}

\section{(Voir encadré texte ci-dessous, à la fin du présent chapitre)}

\section{d. ORGANISATIONS INTERNATIONALES}

\section{(Voir encadré texte dans la section $16 . b$ traitant du rôle des organisations intergouvernementales;} voir également encadrés textes sur APELL aux sections 5.d et 16.b)

\section{e. ORGANISATIONS NON GOUVERNEMENTALES (ONG)}

4.e.1 Les ONG (comme les groupes environnementaux, humanitaires et de consommation) devraient encourager leurs éléments constitutifs et d'autres à s'engager dans la voie de la réduction des risques et de la prévention des accidents. Ils devraient aider à déterminer 
les préoccupations et les priorités spécifiques concernant la réduction des risques ainsi que les activités de prévention, de préparation et d'intervention.

4.e.2 Les ONG devraient faciliter les mesures visant à informer le public, et fournir l'aide technique pour permettre au public d'analyser et de comprendre l'information disponible.

4.e.3 Les ONG devraient avoir, le cas échéant, la possibilité de contribuer aux processus décisionnels concernant les installations dangereuses, comme ceux s'appliquant à la délivrance de licences et à l'aménagement du territoire. Les membres des ONG pourraient avoir les qualifications et l'expérience leur permettant d'examiner l'information technique, les documents juridiques et autres matériels nécessaires pour une participation effective et pour la recommandation de possibles solutions aux préoccupations constatées.

- 4.e.4 Les ONG devraient, le cas échéant, participer aux processus de législation et de réglementation, par exemple en aidant à déterminer les préoccupations du public qui pourraient influer sur les objectifs des politiques, en fournissant des analyses de l'information provenant d'un ensemble d'installations (p. ex. concernant des cas concrets $\mathrm{d}$ 'incidents), en proposant de nouvelles directives en matière de politiques, et enfin en s'inspirant de l'expérience acquise par d'autres pays ou régions. 


\section{ÉTABLISSEMENTS DE RECHERCHE UNIVERSITAIRES OU AUTRES}

On devrait encourager les établissements de recherche universitaires ou autres à entreprendre des recherches sur la prévention, la préparation et l'intervention en matière d'accidents chimiques.

Les activités considérées comme utiles pour des recherches additionnelles dans ce domaine sont les suivantes :

- améliorer les techniques d'évaluation des risques et évaluer les possibilités d'accidents chimiques et la capacité d'intervention grâce à un meilleur équipement et à de meilleures pratiques;

- chercher à mieux comprendre les effets sur la santé et l'environnement attribuables à des expositions aiguës à différentes substances dangereuses*;

- améliorer le traitement médical et les procédures de décontamination;

- chercher à mieux comprendre l'acceptabilité/tolérabilité en matière de risques.

Les organisations engagées dans des activités de recherche sur la prévention, la préparation et l'intervention en matière d'accidents chimiques devraient coopérer et partager les résultats de leurs recherches afin d'établir un climat de confiance au niveau des résultats et éviter les chevauchements. La coopération peut être facilitée grâce à des projets de recherche et à des réseaux d'information multipartites avec partage des coûts, ainsi qu'à l'échange d'information aux réunions et par de la documentation.

Pour partager le plus largement possible les résultats des recherches liées à la sécurité, ces derniers devraient être disponibles dans un format et dans une langue facilement compréhensibles par les utilisateurs potentiels.

De plus, on devrait constituer des inventaires nationaux et internationaux des activités de recherche afin de faciliter la diffusion de l'information et des résultats en matière de recherches.

Dans un domaine connexe, il est recommandé que le programme de cours et de recherches des facultés de sciences et d'ingénierie des universités et écoles comprenne, comme discipline pleinement intégrée, l'analyse des risques et d'autres aspects liés à la sécurité au niveau de la conception, de l'exploitation et de la gestion des installations dangereuses et du transport de substances dangereuses. Ce sujet sera plus amplement élaboré à un atelier de l'OCDE prévu pour 2003.

De plus, des cours en planification pour les urgences, en sécurité et en analyse des risques devraient faire partie des programmes de vulgarisation et d'éducation permanente.

Enfin, les organisations chargées de recherches devraient établir des liens avec l'industrie afin de déterminer des sujets de recherche pertinents à l'intention des étudiants diplômés.

*Des travaux sur les conséquences pour la santé sont en cours dans le cadre de plusieurs projets nationaux et internationaux, comme le projet américain AEGL (« Acute exposure guideline levels») et le projet ACUTEX de la CE visant à élaborer des méthodes innovatrices pour définir les niveaux d'exposition aiguë qui pourraient être utilisés pour établir les concentrations d'exposition aiguë à des fins de planification pour les situations d'urgence et l'aménagement du territoire. 


\section{NOTES}

1. Chaque pays et chaque entreprise peut avoir un mode différent de représentation des employés, notamment en ce qui concerne les représentants syndicaux et les représentants en sécurité.

2. Aux fins de la présente publication, les incidents sont définis comme étant des accidents ou des quasi-accidents.

3. Aux fins de la présente publication, les employés, selon la définition de l'annexe 1, comprennent à la fois la direction et les travailleurs, ainsi que les contractants/sous-traitants.

4. Dans certains pays, ces rapports sont appelés « rapports sur la sécurité » ou «plans de gestion des risques ».

5. Ces étapes peuvent être décrites à l'aide d'une terminologie différente. Par exemple, dans le thésaurus CARAT (système utilisant Internet, élaboré par l'OCDE pour faciliter la communication concernant l'évaluation du risque), les éléments généraux sont décrits comme suit : identification du danger (hazard identification); analyse de l'exposition aux rejets (release exposure analysis); effets de la dose (dose response); expression du risque (risk expression), suivi d'intégration du risque (risk integration). Pour plus de renseignements sur le thésaurus CARAT, voir la note associée au paragraphe 2.b.9.

6. Suite aux craintes voulant qu'un manque de cohérence dans les définitions des termes clés nuise au partage de l'expérience dans le domaine de l'évaluation du risque, l'OCDE a mis au point un système en ligne, appelé CARAT (Chemical Accident Risk Assessment Thesaurus). Le CARAT a été créé pour faciliter la communication concernant l'évaluation des risques d'accident chimique et, à l'intérieur de chaque pays, aider à surmonter les problèmes entraînés par les différences de culture et de langue. Le CARAT est un système qui réunit, dans une base de données, l'information sur différentes méthodes d'utilisation des lois, des règlements, des documents d'orientation, des études de cas et de la terminologie associés à l'évaluation des risques. Le système CARAT est fondé sur le principe voulant que le processus d'évaluation du risque comporte diverses étapes qui peuvent être décrites dans une langue opérationnelle objective. Cette langue opérationnelle possède la qualité de la transparence et constitue une base pour la comparaison entre les différentes méthodes. Les utilisateurs ont accès à la base CARAT via Internet; ils peuvent y visualiser chaque entrée, comparer deux ou plusieurs entrées, et effectuer diverses recherches destinées aux utilisateurs. L'adresse Internet du CARAT est www.oecd.org/ehs/carat.

7. Les expressions « sécurité intrinsèque » ou « intrinsèquement sûr » utilisées dans le contexte des installations dangereuses ne devraient pas être interprétées au sens d'absence totale de risque résiduel. Il est important d'utiliser ces expressions de façon prudente; les risques présents à l'installation doivent être bien compris et les communications à ce sujet doivent être adéquates.

8. Il y a effet domino lorsqu'un accident entraîne des effets nuisibles plus importants ou déclenche d'autres accidents en raison de la proximité d'autres parties de l'installation ou d'installations voisines et de leurs inventaires de substances dangereuses.

9. L'OCDE a publié en 2003 des notes d'orientation sur l'élaboration et l'application d'indicateurs de performance en matière de sécurité à l'intention des pouvoirs publics, de l'industrie et de la communauté. 
10. Voir, par exemple, les annexes pertinentes de la Directive «Seveso II » et de la Convention sur les effets transfrontières des accidents industriels de la CEE-ONU.

11. Dans certains pays, ces rapports sont appelés « rapports sur la sécurité » ou «plans de gestion des risques».

12. L'information cruciale pour la sécurité ne doit en aucun cas être considérée comme un secret commercial.

13. L'OCDE a publié en 2003 des notes d'orientation sur l'élaboration et l'application d'indicateurs de performance en matière de sécurité à l'intention des pouvoirs publics, de l'industrie et de la communauté. 
Partie B

\section{PRÉPARATION AUX SITUATIONS D'URGENCE ET ATTÉNUATION DE LEURS CONSÉQUENCES}

Cette partie porte sur les rôles et responsabilités des différentes parties prenantes au chapitre de la planification pour les situations d'urgence et de l'atténuation des conséquences des accidents (ce qui englobe l'aménagement du territoire et l'information du public).

Comme il sera expliqué ci-après, la responsabilité de préparer les plans d'urgence internes incombe tout d'abord à l'industrie, alors que celle de mettre en place les plans d'urgence externes revient principalement aux pouvoirs publics. Cependant, le processus de planification pour les situations d'urgence requiert, pour être efficace, une coopération entre les diverses parties prenantes, notamment le personnel d'intervention, le personnel médical et paramédical, les représentants du public et les médias.

Aux fins du présent document, sont comprises dans le personnel médical et paramédical tant les organisations que les personnes qui détiennent des responsabilités au chapitre de la santé publique. 


\section{Chapitre 5}

\section{PRÉPARATION ET PLANIFICATION POUR LES SITUATIONS D’URGENCE}

\section{a. PRINCIPES GÉNÉRAUX}

Cette section vise la planification pour les situations d'urgence (également appelée planification pour les urgences ou simplement planification dans le présent chapitre) tant à l'installation qu'à l'extérieur de celle-ci.

5.a.1 Les pouvoirs publics (à tous les paliers) et la direction des installations dangereuses devraient mettre en oeuvre des activités ou des programmes relatifs à la planification pour les situations d'urgence liées à des accidents mettant en cause des substances dangereuses.

" Les activités et les programmes de planification applicables aux cas d'urgence devraient permettre de mettre en place les dispositions nécessaires pour localiser tous les accidents et, si possible, pour les maîtriser afin d'atténuer leurs effets nuisibles sur la santé, l'environnement et les biens.

" Le recensement des installations dangereuses présentes dans le secteur visé par le plan d'urgence est une condition préalable à la planification d'urgence.

" Il faudrait préparer des plans d'urgence internes et externes établissant en détail les procédures techniques et administratives pour réduire au minimum les effets sur la santé, l'environnement et les biens (tant sur les lieux mêmes qu'à l'extérieur) en cas d'accident.

" La planification pour les situations d'urgence intéressant les installations dangereuses devrait prendre en compte les autres risques, comme ceux d'accidents pendant le transport de substances dangereuses et les catastrophes naturelles.

5.a.2 Il faudrait, dans le cadre de la planification pour les urgences, élaborer les scénarios envisageables et caractériser les risques potentiels ainsi que les zones géographiques où des effets sont susceptibles de se produire en cas d'accident. Il faudrait notamment préciser, pour ces zones, quelles populations risquent d'être touchées et dans quels secteurs il est possible qu'on doive prendre la décision d'évacuer, de recommander aux gens de se mettre à l'abri sur place ou d'appliquer d'autres mesures pour limiter l'exposition humaine. Il faudrait également indiquer, pour ces zones, la nature et l'ampleur des ressources qui pourraient être requises en cas d'accident.

" Au moment de déterminer les zones d'exposition probable, il faudrait prendre en considération la possibilité que des effets nuisibles puissent s'exercer par suite : d'un contact direct avec des substances toxiques ou irritantes (à titre d'exemple, par contact oculaire, exposition cutanée ou inhalation); d'un rayonnement thermique ou $d^{\prime}$ une surpression; d'une exposition indirecte (par exemple, par ingestion d'eau ou de nourriture contaminée); de blessures indirectes (causées par l'effondrement de structures, par des projectiles ou un incendie) $)^{1}$. 
" Au moment de déterminer les zones où des effets pourraient s'exercer, il faudrait indiquer s'il s'y trouve des infrastructures critiques (dont des services de transport et des routes), des milieux écologiquement sensibles et des établissements abritant des populations vulnérables (comme les hôpitaux, les maisons de retraite et les maisons de repos, les centres commerciaux, les écoles et autres lieux de rassemblement des enfants). En ce qui concerne les établissements où se trouvent des populations vulnérables, il pourrait être nécessaire, en cas d'accident, de se rendre sur les lieux mêmes pour donner des instructions spécifiques.

" Il faudrait prendre ces zones en compte dans le processus de planification pour les urgences. Il faudrait déterminer les répercussions possibles en se fondant sur le pire des scénarios d'accidents et sur le scénario d'accident le plus probable.

"Lorsqu'un accident se produit, il faut déterminer les zones qui sont véritablement à risque d'après la nature des substances dangereuses qui s'échappent de l'installation en cause, les conditions météorologiques et les vents dominants, et la dispersion probable des substances dans l'environnement.

5.a.3 Le processus de planification pour les urgences devrait comprendre l'estimation des conséquences potentielles des accidents pour l'environnement et la santé, et prévoir les mesures appropriées de prévention, de préparation et d'intervention.

" Si un accident survient à une installation dangereuse, la planification pour les urgences devrait viser à éviter la pollution des milieux environnementaux, comme les sources d'eaux de surface et souterraines ainsi que les sols.

" En outre, toutes les évaluations environnementales relatives à des projets d'aménagement devraient tenir compte, s'il y a lieu, de la possibilité d'accidents chimiques.

\section{(Voir la section $2 . b$ sur l'identification des dangers et l'évaluation des risques)}

5.a.4 La planification pour les urgences devrait tenir compte des possibles facteurs de complication associés aux accidents liés à des installations dangereuses ainsi que des éléments susceptibles de rendre l'intervention plus difficile. Parmi ces facteurs et éléments figurent notamment les conditions météorologiques extrêmes, les catastrophes naturelles, la coupure de l'approvisionnement en énergie ou en eau, les problèmes touchant les réseaux de communication et de transport, les effets de synergie s'exerçant lors d'accidents qui mettent en cause plusieurs substances dangereuses, les effets dominos ou encore du sabotage.

5.a.5 Toutes les parties qui seront associées à l'intervention d'urgence (par exemple, les pompiers et les policiers) devraient participer au processus de planification pour les urgences.

" À ce chapitre, les autorités responsables de la santé publique, y compris les spécialistes provenant des centres d'information, devraient prendre part à tous les segments pertinents de la planification pour les urgences à l'installation et à l'extérieur de celle-ci.

"Il faudrait offrir aux représentants du public l'occasion de participer au processus de planification. 
" Les représentants des médias devraient également être associés à l'élaboration des plans d'urgence.

\section{(Voir la partie C sur l'intervention d'urgence)}

5.a.6 Les plans d'urgence externes et tous les plans d'urgence internes applicables devraient être cohérents et intégrés.

4I Il s'agit là d'un élément essentiel si l'on veut :

- que la coordination soit efficace;

- que les problèmes relatifs au chevauchement des responsabilités et aux interfaces complexes soient surmontés;

- que le partage des responsabilités soit clair quant aux divers aspects de l'intervention d'urgence en cas d'accident mettant en cause des substances dangereuses pouvant entraîner des effets à l'extérieur des installations.

" Les responsables des plans d'urgence internes devraient travailler en étroite collaboration avec les personnes en charge des plans externes.

5.a.7 Les pouvoirs publics et l'industrie devraient coopérer au chapitre de la planification afin de protéger les zones de peuplement ainsi que les milieux écologiquement sensibles se trouvant à proximité des installations dangereuses.

" Les plans d'urgence devraient comporter des conseils sur les circonstances dans lesquelles le public susceptible d'être touché devrait s'abriter à l'intérieur, et celles où il convient de procéder à une évacuation.

" Les plans d'urgence devraient tenir compte de la situation particulière des établissements locaux dotés d'infrastructures sensibles ou pouvant abriter des populations particulièrement vulnérables, comme les écoles, les hôpitaux, les résidences pour personnes âgées et les centres de détention.

5.a.8 Une bonne coopération entre l'industrie et le personnel d'intervention (par exemple, les services d'incendie, de secours, de police et de santé) est essentielle pour que la préparation et l'intervention soient efficaces.

1 Dans cette perspective, il faudrait que les intervenants soient en mesure de communiquer efficacement (c'est-à-dire qu'ils doivent « être sur la même longueur d'onde »).

" L'industrie et les autorités responsables des services d'urgence devraient partager leurs connaissances et leur expérience concernant tous les aspects de la prévention, de la préparation et de l'intervention.

- 5.a.9 L'industrie, les pouvoirs publics ainsi que les services médicaux et sanitaires devraient coopérer afin de s'assurer que le personnel médical et paramédical - susceptible de participer à une intervention d'urgence liée à des substances dangereuses - est au fait des substances dangereuses produites, utilisées, transportées ou manutentionnées en grandes quantités au sein de la collectivité. Le personnel médical et paramédical devrait également connaître les aspects des plans d'urgence locaux qui le concernent et les rôles qui lui sont assignés dans ces plans. 
(Voir les paragraphes 5.c.11 à 5.c.19 sur les aspects médicaux et paramédicaux de la planification en cas d'urgence)

5.a.10 Les plans d'urgence (tant internes qu'externes) devraient définir les rôles et responsabilités de toutes les parties concernées et déterminer la chaîne de commandement, les voies de communication, la coordination attendue entre les parties et les moyens d'obtenir les renseignements, les ressources et l'équipement nécessaires.

5.a.11 Les plans d'urgence devraient fournir les règles d'orientation qui sont requises pour adapter l'intervention en fonction des diverses situations pouvant se produire (des petits accidents au pire des scénarios d'accidents).

" Un plan d'urgence ne peut donner des instructions précises concernant l'intervention, puisque les accidents sont tous de nature différente et mettent souvent en jeu une combinaison d'éléments qui n'ont peut-être pas été envisagés durant le processus de planification.

D Les plans d'urgence, même s'ils sont valables sur papier, peuvent échouer pour diverses raisons lorsqu'ils sont mis en pratique, notamment les suivantes: défaut d'avoir prévu les déficiences dans les informations disponibles au moment de l'accident; formation insuffisante; coordination insuffisante; rupture des communications; défaut d'admettre les limites des ressources humaines en situation de stress; plan d'une complexité trop grande, ou assignant des tâches trop lourdes à certains intervenants.

5.a.12 Les plans d'urgence internes et externes devraient être mis à l'essai, réexaminés régulièrement et mis à jour au besoin compte tenu, par exemple, des changements dans la nature des risques, des nouveaux aménagements résidentiels et commerciaux dans le secteur, du perfectionnement des techniques et des capacités d'intervention, des leçons tirées des exercices ou des essais ainsi que de l'application des plans d'urgence lors d'accidents ou de quasi-accidents, et enfin du changement de personnel.

\ Il est primordial de mettre les plans d'urgence à l'essai périodiquement pour s'assurer qu'ils sont adéquats, complets et réalistes, et que les différents plans applicables dans un secteur donné (à l'installation et à l'extérieur de celle-ci) sont compatibles. Cela permet également de relever les déficiences ou les besoins au niveau de la disponibilité en personnel approprié (y compris les besoins en formation) ainsi qu'en équipement, en fournitures et en information nécessaires. En outre, tester les plans d'urgence renforce la confiance du personnel d'intervention en ses capacités de répondre à des situations d'urgence réelles.

" Les exercices peuvent mettre à l'essai différents segments du plan isolément et peuvent comprendre des simulations, par exemple sur table, par ordinateur.

" Il faut établir une fréquence d'essais appropriée et déterminer quels segments devraient être testés à un moment donné (vu que tous les segments du plan ne seront pas soumis à chaque essai). On peut, par exemple, mettre à l'essai en priorité les secteurs incertains ou ceux qui n'ont pas fait l'objet d'essais depuis quelque temps.

* Certains exercices devraient être menés dans des conditions défavorables (par exemple, en dehors des heures normales de travail, par mauvais temps, etc.) pour 
mettre en lumière l'éventail des limites et des problèmes associés aux plans d'urgence.

"Les personnes qui seront mobilisées en cas d'accident devraient participer aux essais ou exercices. À titre d'exemple, comme l'intervention en cas d'accident exigera que des décisions soient prises par de hauts responsables (de l'industrie et des pouvoirs publics), ceux-ci devraient prendre part aux exercices pertinents. En outre, les membres du public devraient être associés aux parties des exercices ou des essais qui les concernent.

" Si les manœuvres prévues dans le cadre d'un exercice ou d'un essai sont susceptibles de soulever des questions ou de faire naître des préoccupations chez le public, l'exercice ou l'essai devrait être expliqué à l'avance.

" Les exercices ou les essais les plus profitables sont ceux pendant lesquels on s'abstient de formuler des reproches (c'est-à-dire que personne n'est blâmé pour les erreurs ou les problèmes relevés). Dans un tel climat, tous les participants se sentiront libres de s'exprimer de façon ouverte et honnête dans leurs évaluations, sans craindre des conséquences fâcheuses.

" En recourant à des observateurs indépendants durant les exercices, on facilitera l'examen des déficiences et des imperfections relatives aux plans d'urgence en toute objectivité.

" En ce qui concerne la mise à l'essai des plans d'urgence externes, il faudrait envisager des essais conjoints des plans applicables à un secteur donné (par exemple, lorsqu'il existe plus d'une installation dangereuse dans le secteur, ou lorsqu'un éventuel accident pourrait toucher plus d'une collectivité d'un même pays ou de pays différents). Il s'agit là d'une manière de procéder économique et propre à fournir un bon aperçu des faiblesses de la planification.

4 Les essais devraient également concerner le transport de substances dangereuses entrepris sous la responsabilité des installations dangereuses.

" Les résultats des exercices ou des essais relatifs aux plans d'urgence ainsi que les plans d'urgence révisés devraient être publiés et diffusés largement afin d'informer tous ceux qui peuvent avoir un rôle à jouer en cas d'accident et de permettre à d'autres parties de profiter de l'expérience.

5.a.13 Au cours de l'élaboration des plans d'urgence, il faudrait procéder à une évaluation réaliste des compétences, de l'équipement et des autres ressources auxquels on pourra recourir en cas d'intervention, ainsi qu'à une évaluation des compétences, de l'équipement et des autres ressources qui sont requises, en se basant sur l'éventail de scénarios d'accidents envisageables, y compris le pire des scénarios. Ces évaluations donneront un aperçu des lacunes en matière de compétences, d'équipement et d'autres ressources.

5.a.14 Toutes les parties responsables devraient veiller à ce que les ressources humaines, les ressources financières, l'équipement (y compris le matériel de communication et l'équipement de protection individuelle) ainsi que les autres ressources nécessaires pour appliquer les plans d'urgence soient facilement accessibles et puissent être mis en œuvre immédiatement en cas d'accident ou de menace imminente d'accident. Au besoin, il 
faudrait se procurer l'équipement spécialisé ou très coûteux par l'intermédiaire de programmes de coopération avec d'autres collectivités, avec les pouvoirs publics d'autres paliers, ou encore avec l'entreprise privée.

" Il faudrait établir des mécanismes d'aide mutuelle entre les collectivités voisines ou les autres collectivités concernées au sein d'un même pays ou de part et d'autre d'une frontière au cas où l'intervention nécessitée par un accident dépasserait les capacités locales.

" Les pouvoirs publics des collectivités voisines (au sein d'un même pays ou de part et d'autre d'une frontière) devraient mettre leurs ressources en commun (y compris l'équipement, les compétences spécialisées ainsi que les ressources et l'information médicales) afin d'employer au mieux les capacités d'intervention. Il faudrait tâcher de faire en sorte que l'équipement et autres ressources pertinentes qui sont partagés avec d'autres collectivités soient compatibles (par exemple, les raccords de tuyauterie).

" Avant d'envisager d'offrir des ressources à d'autres collectivités dans un but d'entraide, il est important que les collectivités se ménagent des capacités d'intervention suffisantes et des ressources d'intervention à la hauteur des risques locaux.

" Il faudrait s'assurer qu'il existe des capacités d'intervention adéquates à l'échelle nationale, de sorte qu'une quantité raisonnable de ressources, en additionnant les ressources publiques et privées, soit disponible dans chaque secteur compte tenu du niveau de risque. Dans certaines régions, il pourrait être nécessaire que les entreprises fournissent de l'équipement et des ressources pour intervenir en cas d'accident lié à leurs installations dangereuses, cela afin de compenser le manque de ressources chez les autorités locales.

\section{(Voir la section 16.a sur la coopération transfrontalière)}

5.a.15 Il faudrait que le personnel d'intervention ait facilement accès à l'information, aux fournitures et à l'équipement dont il a besoin pour évaluer l'ampleur d'un accident et décider des mesures d'intervention appropriées.

" Il devrait notamment disposer de méthodes analytiques et de matériel de détection des substances dangereuses, d'équipement de protection en cas de fuite ou de déversement, et d'information sur les premiers soins et les antidotes à administrer pour contrer les effets de certains produits chimiques.

"L'information technique contenue dans les plans d'urgence (par exemple, les propriétés physico-chimiques des substances) devrait être présentée sous une forme qui convienne aux intervenants en cas d'urgence et devrait proposer des orientations claires quant aux mesures à prendre.

"L'information concernant les possibles effets néfastes des accidents - déduits des scénarios envisagés et des modèles de dispersion - devrait être mise à la disposition des intervenants en cas d'urgence en temps réel, de manière à permettre une intervention rapide. 
" Il faudrait pouvoir trouver sur place, dans toutes les installations dangereuses, des renseignements clairs sur la sécurité, notamment de l'information sur ce qu'il faut faire en cas d'accident, la façon de réduire les répercussions néfastes sur la santé, l'environnement et les biens et les premiers soins à donner aux personnes exposées.

" Il faudrait s'employer à traiter le problème des substances dangereuses ayant fait l'objet de peu d'évaluations (et pour lesquelles, en conséquence, les mesures d'intervention en cas d'accident sont mal connues). De plus, ces substances devraient constituer une priorité en matière de recherche afin d'améliorer la connaissance de leurs effets possibles sur la santé et sur l'environnement ainsi que des traitements à appliquer pour contrer ces effets.

5.a.16 Il faudrait prévoir des systèmes auxiliaires dans les plans d'urgence. Par exemple, il faudrait disposer de voies de communication de remplacement, $d^{\prime}$ une équipe de relève des intervenants clés, et d'un second quartier général au cas où le premier ne puisse fonctionner correctement.

5.a.17 Il devrait exister des systèmes et des procédures pour détecter rapidement les accidents ou les menaces imminentes d'accident et alerter immédiatement le personnel d'intervention d'urgence.

5.a.18 Le processus de planification d'urgence devrait comprendre l'élaboration de méthodes pour informer le public des mesures à prendre en cas d'urgence, et de la façon dont il sera avisé qu'un accident s'est produit, le cas échéant.

" Il devrait exister des systèmes d'alarme en cas d'urgence pour avertir le public susceptible d'être touché qu'un accident s'est produit, ou qu'il y a menace imminente d'accident.

" Le système choisi devrait être efficace et permettre de donner l'alerte rapidement. Il peut s'agir de sirènes, de messages téléphoniques automatisés ou de systèmes de hauts-parleurs mobiles, ou encore d'une combinaison de ces éléments.

" Le public pouvant être touché devrait être avisé des systèmes qui seront utilisés pour l'avertir en cas d'urgence. En outre, ces systèmes devraient être éprouvés à l'avance, de sorte que le public en comprenne l'importance et sache comment réagir adéquatement en cas d'urgence.

\section{(Voir le chapitre 7 sur l'information du public)}

5.a.19 Les porte-parole désignés en prévision des situations d'urgence devraient être choisis avec soin durant le processus de planification; il convient en effet de s'assurer qu'ils possèdent les compétences, l'autorité et la crédibilité requises pour communiquer efficacement avec le public.

" Les porte-parole devraient être spécialement sélectionnés et formés de sorte qu'ils sachent comment présenter l'information en fonction du public cible et comment la transmettre efficacement.

" Pour être efficace, la communication avec le public en situation d'urgence requiert la participation coordonnée d'un certain nombre de parties concernées - dont, par exemple, les responsables locaux en matière d'intervention, les porte-parole des 
entreprises, les représentants des employés, les représentants des collectivités, les pouvoirs publics, les spécialistes techniques et les médias —; c'est pourquoi les tâches de chacune de ces parties devraient être définies lors de la préparation des plans d'urgence.

\section{(Voir paragraphe 7.13 sur le rôle des médias)}

5.a.20 Les pouvoirs publics, l'industrie et les autres parties prenantes devraient, s'il y a lieu, participer à des activités de coopération internationales et régionales concernant la planification en cas d'urgence dans le but de partager leur expérience, d'améliorer la planification et de faciliter la coordination voulue lors des interventions effectuées en cas d'accident.

\section{b. INDUSTRIE}

- 5.b.1 Toutes les installations dangereuses devraient se doter d'un plan d'urgence interne adéquat et établi d'après un éventail complet de scénarios d'accidents, y compris le pire des scénarios et les cas de rejets les plus probables.

D Afin de donner une assise à la planification en cas d'urgence (plans d'urgence externes comme internes), la direction des installations dangereuses - y compris les PME et les entreprises qui ne font pas partie de l'industrie chimique, mais où sont utilisées ou manutentionnées des substances dangereuses - devrait déterminer et évaluer toute la gamme d'accidents qui pourraient se produire aux installations (y compris les accidents dont la probabilité est faible, avec des conséquences graves), puis établir les mesures d'intervention appropriées. Cette information devrait se trouver dans les « rapports sur la sécurité » ou dans les rapports apparentés, si de tels documents sont rédigés.

\section{(Voir les paragraphes 2.a.16 à 2.a.18 concernant les rapports sur la sécurité)}

" Les plans d'urgence internes devraient contenir un plan à l'échelle du site, accompagné d'une liste des substances manutentionnées indiquant les quantités en jeu et leur emplacement sur le site par rapport aux populations et zones voisines. Les plans d'urgence devraient également comprendre une évaluation des dangers et présenter de l'information au sujet de chacune des substances dangereuses, des conditions dans lesquelles elles sont fabriquées, manutentionnées et entreposées, et des premiers soins à prodiguer aux employés et aux membres du public s'ils sont exposés à l'une de ces substances dangereuses.

D Les plans d'urgence internes devraient également énoncer des instructions permettant au besoin d'interrompre, en phases séquencées, les activités de l'installation.

- La direction devrait être responsable de la préparation d'un plan d'urgence interne, et l'entreprise à qui appartiennent les installations devrait financer cette démarche. La responsabilité de mettre le plan en œuvre devrait incomber à la direction, avec l'appui d'autres employés.

Les plans d'urgence internes devraient être mis à la disposition des pouvoirs publics à des fins de réexamen. 
" Toute installation dangereuse ne disposant pas des ressources nécessaires pour procéder à la planification en cas d'urgence, compte tenu de l'éventail complet des accidents qui pourraient se produire à l'installation, devrait demander de l'aide (auprès, par exemple, des installations voisines, des fournisseurs ou des pouvoirs publics) pour remplir ses obligations.

5.b.2 Les plans d'urgence internes devraient énoncer les rôles et responsabilités de toutes les parties concernées, et devraient déterminer clairement la chaîne de supervision, la coordination entre les parties, les voies de communication et les moyens d'obtenir l'information requise.

" Dans le cadre de la préparation des plans d'urgence internes, il faudrait nommer des personnes aux postes suivants :

- coordonnateurs internes (de l'entreprise), devant prendre en charge, le cas échéant, les lieux de l'accident;

- responsables en chef sur place, issus du centre d'urgence de l'entreprise, devant prendre la tête des opérations en cas d'urgence et s'occuper de la communication avec les pouvoirs publics;

- responsable du déclenchement des plans d'urgence externes.

" Le rôle des coordonnateurs internes et des responsables en chef sur place par rapport à celui du personnel d'intervention d'urgence de la collectivité devrait être clairement énoncé, de manière à éviter tout conflit potentiel. À ce chapitre, les coordonnateurs internes et les responsables en chef pourraient avoir à se conformer aux décisions prises par le coordonnateur sur les lieux de l'accident (coordonnateur sur place) désigné par les pouvoirs publics.

" Les plans d'urgence internes, lorsqu'il s'agit de déterminer les responsabilités des divers employés ${ }^{2}$ en cas d'accident, devraient être empreints d'une certaine souplesse, de façon à pouvoir s'appliquer en dépit des variations prévisibles dans l'occupation des postes, et devraient tenir compte d'éléments comme les absences pour cause de maladie, de vacances ou de fermeture des installations.

- 5.b.3 Tous les employés des installations dangereuses devraient connaître parfaitement les dispositions pertinentes du plan d'urgence interne. Ils devraient notamment savoir quoi faire en cas d'accident, par exemple prendre des mesures pour limiter les rejets de substances dangereuses et (ou) évacuer les lieux et se rendre à un point de rassemblement déterminé à l'avance.

" Tous les employés devraient être informés des procédures pour donner l'alarme en cas d'accident ou de menace imminente d'accident, cela afin que des mesures soient prises aussi rapidement que possible pour maîtriser l'incident.

" La direction devrait s'assurer que les employés concernés sont au courant des capacités et des plans d'intervention des services d'incendie et autres intervenants en cas d'urgence.

5.b.4 Les visiteurs qui pénètrent dans des installations dangereuses devraient être informés de ce qu'ils doivent faire en cas d'urgence. 
5.b.5 L'industrie ne devrait pas invoquer le motif du secret de fabrication pour refuser de divulguer au public des informations concernant la sécurité de l'usine, les mesures de sécurité et les caractéristiques des substances en jeu. En règle générale, les multinationales ne devraient pas invoquer, dans un pays donné, la protection des secrets de fabrication comme motif pour retenir des renseignements qu'elles diffusent pourtant dans un autre pays. Toute exception à ce principe devrait être justifiée au cas par cas.

5.b.6 La direction des installations dangereuses devrait s'assurer de conserver, en un lieu facilement accessible, des quantités suffisantes de fournitures médicales d'urgence nécessaires (y compris les antidotes appropriés), et de les renouveler au besoin. Cela revêt une importance particulière dans les secteurs où il est difficile pour les autorités sanitaires locales de maintenir de tels stocks.

5.b.7 Les activités touchant la préparation en cas d'urgence et la prévention des accidents chimiques devraient être intégrées au fonctionnement normal des installations dangereuses, de sorte que les structures organisationnelles rattachées à ces deux types d'activités soient compatibles.

5.b.8 La direction devrait travailler avec les pouvoirs publics à l'élaboration des plans d'urgence externes pour s'assurer que les responsables de la préparation de ces documents ont en main tous les renseignements qu'il leur faut, y compris l'information nécessaire à l'évaluation des dangers, et pour veiller à la compatibilité des plans d'urgence internes et externes.

Dans le cadre du processus de planification en cas d'urgence, la direction devrait, en collaboration avec les intervenants d'urgence, examiner les possibilités d'intervention pour les différents scénarios d'accidents et s'entendre sur les démarches qu'il convient d'adopter pour les divers scénarios.

- Aux fins de la préparation, de l'intervention et du suivi applicables aux urgences, la direction devrait publier les informations relatives à la santé concernant les substances dangereuses fabriquées et (ou) distribuées, entreposées, manutentionnées, transformées, éliminées ou utilisées de quelque autre façon sur le lieu de travail. Cela comprend les renseignements touchant la composition de même que les propriétés toxicologiques, écotoxicologiques et autres des substances dangereuses, y compris les solvants et les additifs.

En plus de fournir les renseignements relatifs aux installations et aux produits chimiques en jeu sur place, la direction devrait coopérer avec les pouvoirs publics pour le choix du tracé et le relevé des pipelines ainsi que des itinéraires routiers empruntés à l'extérieur du périmètre des installations pour acheminer des substances dangereuses en provenance ou à destination des installations.

- La direction ne devrait jamais dissimuler des informations nécessaires à l'élaboration des plans d'urgence. Ainsi, il ne faudrait jamais invoquer le motif du secret de fabrication ou de la confidentialité des renseignements commerciaux pour omettre de révéler ces informations. Cependant, il faudrait, s'il y a lieu, prendre des dispositions pour que toute personne recevant des renseignements commerciaux en préserve la confidentialité. 
5.b.9 L'industrie devrait coopérer avec les pouvoirs publics et s'assurer que les populations pouvant être touchées possèdent l'information adéquate pour comprendre les risques encourus et les mesures à prendre en cas d'accident. La direction et les employés des autres échelons, aux installations dangereuses, devraient entretenir d'étroites relations avec les dirigeants communautaires, les établissements d'enseignement et les autres membres de la population locale afin d'aider à accroître la connaissance qu'ont les collectivités des concepts relatifs au risque.

5.b.10 Les entreprises d'une même zone géographique (y compris, par exemple, les entreprises d'une zone portuaire ou d'un parc industriel) devraient coordonner leurs plans d'urgence internes et leurs mesures d'intervention afin de garantir la cohérence de ces plans, d'établir des mécanismes d'aide mutuelle et, le cas échéant, de contribuer à éviter les effets dominos.

\section{c. POUVOIRS PUBLICS}

Cette section englobe les autorités et installations sanitaires et médicales.

5.c.1 Les pouvoirs publics devraient établir des règles d'orientation et des normes applicables à l'élaboration des plans d'urgence internes et externes.

- 5.c.2 Les pouvoirs publics devraient veiller à ce que les plans d'urgence internes et externes soient élaborés, appliqués, mis à l'essai et tenus à jour en collaboration avec la direction des installations dangereuses et, au besoin, avec la participation des employés et des représentants des collectivités concernées, étant entendu que la responsabilité de la préparation et de la mise en œuvre effectives de tels plans n'est pas attribuée de la même façon dans tous les pays.

" Les pouvoirs publics des divers paliers ont des responsabilités au niveau des plans $\mathrm{d}^{\prime}$ urgence internes et externes.

- En règle générale, les autorités centrales (nationales et régionales) devraient énoncer des principes généraux concernant la planification en cas d'urgence et élaborer des règles $\mathrm{d}$ 'orientation quant aux mesures d'intervention et de remise en état des milieux endommagés lors d'un accident. Elles devraient également prodiguer (au besoin) conseils et aide aux autorités locales et veiller à ce que les fonctionnaires de tous les paliers soient déterminés à constituer des capacités appropriées de préparation en cas d'urgence et d'intervention.

- En règle générale, les pouvoirs publics de tous les paliers devraient veiller à ce que des plans d'urgence internes et externes soient élaborés, et que ces plans puissent être mis en œuvre en conformité avec les principes généraux énoncés.

" La responsabilité de la préparation et de la mise en œuvre effectives des plans $\mathrm{d}^{\prime}$ urgence externes peut incomber aux fonctionnaires locaux ou encore à un groupe ou un comité désigné à cet effet, selon les lois et les politiques applicables dans la localité en question. Les autorités régionales ou nationales peuvent également assumer une part de cette responsabilité. Il faudrait cependant que soit clairement indiqué qui détient le pouvoir de décision en matière d'élaboration et d'application des plans d'urgence. 
5.c.3 Les pouvoirs publics devraient veiller à ce que tous les secteurs où se trouve une installation dangereuse soient dotés d'un plan d'urgence externe adéquat.

"Le plan d'urgence externe devrait :

- énoncer les objectifs poursuivis;

- présenter toutes les informations pertinentes sur les installations dangereuses et ses environs;

- établir les procédures à suivre et désigner les représentants officiels responsables en cas d'accident.

" Les pouvoirs publics devraient s'attacher tout particulièrement à vérifier si toutes les installations dangereuses, y compris les PME et les utilisateurs de substances dangereuses à des fins commerciales, évaluent l'éventail complet de scénarios d'accidents possibles et procèdent à une planification appropriée en cas d'urgence. Il faudrait au besoin trouver l'aide nécessaire pour s'assurer que ces entreprises assument leurs responsabilités quant à la planification pour les situations d'urgence.

- 5.c.4 Les pouvoirs publics de tous les paliers devraient incorporer la planification pour les situations d'urgence touchant les installations dangereuses au processus de planification pour les catastrophes naturelles (comme une inondation, un séisme ou une tempête) et aux mesures de protection civile puisque toutes ces activités font intervenir, pour une bonne part, les mêmes exigences. Cette intégration devrait permettre de produire des plans d'urgence coordonnés et cohérents, à l'intérieur d'une structure de supervision harmonisée. Il faudrait garder à l'esprit que les catastrophes naturelles peuvent être à l'origine d'accidents chimiques dans les installations dangereuses, et qu'elles peuvent entraver les efforts d'intervention en cas d'urgence.

5.c.5 Dans le cadre de l'élaboration des plans d'urgence externes, les pouvoirs publics devraient recenser toutes les parties qu'on s'attend à voir participer à l'intervention d'urgence. En outre, il faudrait établir de manière réaliste les rôles, les ressources et les capacités associés à chacune de ces parties, et obtenir un engagement de la part de chacune.

" Parmi les participants à l'intervention d'urgence figurent notamment les suivants :

- les services de police, d'incendie, de transport et d'aide sociale ainsi que les services médicaux (y compris les hôpitaux);

- les organisations de gestion des situations d'urgence ou de protection civile;

- les organisations responsables des services et des travaux publics;

- la direction des installations dangereuses;

- les centres d'information du public;

- les organismes responsables de la santé publique et de l'environnement.

" Les plans d'urgence, lorsqu'il s'agit de déterminer les rôles et les responsabilités de chacune des parties concernées, devraient déterminer clairement la chaîne de supervision, la coordination entre les parties, les voies de communications et les moyens d'obtenir l'équipement, les ressources et les renseignements nécessaires (par exemple, les données techniques, météorologiques et médicales).

" Les plans devraient désigner un coordonnateur en matière d'urgence (coordonnateur sur place) investi de l'autorité nécessaire pour mobiliser les services d'urgence et coordonner leur intervention. 
"Les plans devraient établir sans équivoque qui a le pouvoir de dégager les ressources d'urgence et de les utiliser.

" Les plans d'urgence devraient tenir compte de la possibilité que les objectifs des divers intervenants d'urgence entrent en conflit (par exemple, les services de police pourrait essayer de préserver les éléments révélant qu'une faute a été commise, alors que les services d'incendie et de santé pourraient ignorer cet aspect dans leur lutte pour maîtriser le rejet de substances dangereuses).

5.c.6 Les plans d'urgence et le personnel d'intervention d'urgence devraient prendre en considération le fait que certaines entreprises pourraient ne pas disposer des ressources et des installations nécessaires pour intervenir en cas d'accident mettant en cause des substances dangereuses.

- 5.c.7 Les plans d'urgence devraient comprendre, à l'intention des intervenants de première ligne, des renseignements détaillés concernant, notamment, la façon dont les divers groupes d'intervention (notamment le personnel médical et paramédical) devraient collaborer, et la manière de procéder pour repérer les victimes, les trier et leur prodiguer les premiers soins.

5.c.8 Tout le personnel participant au processus d'intervention d'urgence (y compris, par exemple, les intervenants de première ligne comme les policiers, les pompiers et les ambulanciers) devrait être formé et sensibilisé en continu, de sorte que les effectifs soient toujours prêts à affronter diverses éventualités. Le personnel d'intervention devrait également être associé au processus de planification en cas d'urgence afin qu'il comprenne les mesures devant être prises en cas d'accident.

" Les programmes de formation et de sensibilisation devraient être éprouvés, évalués et révisés, au besoin, en fonction des changements aux plans et dispositions d'urgence, des risques présents dans les collectivités, des ressources disponibles et des autres facteurs pertinents.

1) Les programmes de formation et de sensibilisation devraient au minimum permettre aux intervenants de première ligne de se familiariser avec :

- les plans d'urgence locaux;

- les installations dangereuses présentes dans la collectivité, y compris les résultats de l'évaluation des risques effectuée à ces installations;

- l'obligation de prendre des mesures de protection lorsqu'on intervient sur les lieux d'un accident lié à des substances dangereuses, y compris le port de vêtements et d'équipement de protection;

- les propriétés essentielles des diverses substances dangereuses présentes dans la collectivité, et les moyens d'intervenir en cas d'accident mettant en cause ces substances;

- les dangers de contamination et les procédures de décontamination;

- les mesures spécifiques de premiers soins;

- les possibles effets nuisibles de nature psychologique chez les victimes, les intervenants en cas d'urgence et le public.

" La formation et la sensibilisation concernant l'intervention d'urgence devraient permettre au personnel d'intervention de poser les gestes appropriés pour réduire le plus possible les effets nuisibles des accidents mettant en cause des substances dangereuses sur la santé et l'environnement. Elles devraient également améliorer sa 
capacité à recueillir de l'information au sujet des possibles effets nuisibles sur la santé et l'environnement.

" Étant donné que les accidents mettant en cause des substances dangereuses sont relativement rares, les plans d'urgence devraient tenir compte de la difficulté de maintenir les compétences nécessaires à l'intervention; dans la mesure du possible, ils devraient confier au personnel d'intervention des responsabilités qui correspondent à leurs tâches quotidiennes habituelles (ou qui s'en rapprochent).

" Le personnel médical et paramédical devrait, au besoin, contribuer à la formation des personnes qui n'œuvrent pas dans le domaine de la santé mais qui participeront vraisemblablement à l'intervention d'urgence.

" Les parties prenantes qui peuvent être associées à l'intervention d'urgence (notamment le personnel d'intervention ainsi que le personnel médical et paramédical) devraient participer à une formation et à des exercices conjoints.

5.c.9 Les pouvoirs publics responsables de l'intervention en cas d'urgence, dont les services d'incendie et les services de secours, devraient se familiariser (à l'avance, en prévision des situations d'urgence) avec l'information pertinente sur les installations dangereuses de leur secteur, qui comprend notamment les renseignements relatifs aux propriétés physiques et chimiques (par exemple, les propriétés toxicologiques et écotoxicologiques) et à l'emplacement des substances dangereuses, de même qu'à l'emplacement des points d'approvisionnement en eau, des systèmes d'extinction à mousse et du reste du matériel de lutte contre les incendies présent aux installations dangereuses.

5.c.10 Les pouvoirs publics devraient s'assurer que les intervenants en cas d'urgence ont accès à des sources d'information (notamment aux centres d'information désignés) qui leur fournissent les renseignements nécessaires pour poser un diagnostic sur les personnes souffrant des effets de substances dangereuses, pour traiter ces personnes et les remettre sur pied.

" Ces centres, ou autres sources d'information, devraient pouvoir renseigner les intervenants sur:

- les substances en cause dans l'accident;

- les premiers soins et le traitement médical;

- les établissements médicaux et les moyens de transport des victimes;

- le moment et la façon d'alerter les services essentiels;

- la structure de supervision qui s'applique à l'intervention;

- la liste des spécialistes disponibles.

" Les pouvoirs publics devraient déterminer quelle est la meilleure façon de garantir l'accès aux informations essentielles.

" Les sources d'information pourraient comprendre des centres spécialisés mis sur pied pour organiser la collecte, la compilation et la diffusion des renseignements relatifs à la planification et à l'intervention en cas d'urgence qui concernent l'exposition humaine aux substances dangereuses, par exemple les centres antipoison. Elles pourraient également comprendre des établissements universitaires, des associations industrielles et d'autres sources. Il faudrait tenir à jour la liste des sources d'information relatives aux différents domaines. 
" Les centres ou sources d'information désignés devraient pouvoir fournir les renseignements voulus 24 heures sur 24, tous les jours de l'année.

" Des représentants des centres ou des sources d'information devraient être prêts, si nécessaire, à participer au processus de planification pour les situations d'urgence ou, selon le cas, à y contribuer.

" Si un pays dispose de plusieurs sources ou centres d'information désignés, ceux-ci devraient être en liaison les uns avec les autres.

"Il faudrait promouvoir l'établissement de réseaux entre les sources d'information.

" Les centres ou sources d'information des différents pays devraient partager leurs renseignements et leur expérience.

" Il faudrait tenir à jour une liste des spécialistes, à l'échelle nationale et internationale, dans divers domaines de la préparation et de l'intervention applicables aux urgences. En outre, il devrait exister un répertoire international des groupes de spécialistes qui peuvent se mettre à la disposition des pays ayant besoin d'aide pour répondre à une situation d'urgence ${ }^{3}$.

5.c.11 La planification pour les situations d'urgence devrait tenir compte de toute la gamme d'effets possibles (effets aigus, effets à long terme et effets psychologiques) que peuvent entraîner les accidents chimiques, et il faudrait prévoir des mesures d'intervention pour soulager ces effets chez le personnel d'intervention, les employés et les membres de la collectivité.

" Il faudrait prendre en considération que les effets sur la santé, chez les populations exposées, peuvent être à court terme et (ou) à long terme. Les effets nocifs peuvent se manifester immédiatement après l'accident, ou demeurer à l'état latent pendant un certain temps. Il peut s'agir d'effets tant directs qu'indirects. Il est possible que des effets psychologiques dont l'exposition n'est pas nécessairement responsable apparaissent lors de l'accident ou après.

" La planification pour les situations d'urgence devrait également prévoir des mécanismes permettant d'atténuer le stress vécu par les personnes responsables de gérer la crise, et d'offrir à celles-ci des services d'écoute.

- 5.c.12 Le processus de planification pour les urgences devrait tenir compte de la nécessité de protéger le personnel soignant contre l'exposition aux substances dangereuses.

" Le personnel peut être exposé à de telles substances lorsqu'il manipule des victimes n'ayant pas été convenablement décontaminées, ou à cause de circonstances imprévues, par exemple un changement dans la direction du vent.

" Normalement, le personnel soignant ne devrait pas pénétrer dans les zones contaminées, sauf circonstances exceptionnelles (par exemple, si des procédures de tri des victimes ou de sauvetage s'imposent). Dans de tels cas, le personnel devrait porter un équipement de protection adéquat et être accompagné par le personnel d'intervention; en outre, il faudrait l'empêcher de dépasser les seuils d'exposition établis. 
5.c.13 Les hôpitaux et autres établissements de traitement dont on pourrait solliciter la participation en cas d'accident lié à des substances dangereuses devraient élaborer des plans d'urgence (coordonnés avec les plans d'urgence externes).

" Ces plans devraient décrire les systèmes ou procédures à appliquer pour admettre et traiter un grand nombre de patients à la fois.

" Ces systèmes ou procédures devraient avoir trait, par exemple, au tri des patients ainsi qu'aux dispositions relatives à leur identification et à leur dossier, et à la décontamination éventuelle.

" Les pouvoirs publics devraient veiller à ce que de tels plans soient en place, et devraient faciliter leur élaboration.

(Voir l'encadré texte suivant le paragraphe 5.c.19, sur la planification en cas d'urgence dans les établissements médicaux)

- 5.c.14 Dans le cadre du processus de planification pour les urgences, il faudrait évaluer les types de ressources médicales d'urgence requises pour répondre aux différents cas d'urgence et soigner toute la gamme de victimes possibles.

- 5.c.15 Dans le cadre du processus de planification pour les urgences, il faudrait vérifier si les établissements médicaux sont adéquats, y compris si les moyens de transports existent.

" Il faudrait disposer, sur les lieux de l'accident et dans les hôpitaux, de matériel de décontamination et, si nécessaire, d'équipement de protection pour le personnel médical d'intervention d'urgence.

Les pouvoirs publics, en collaboration avec les hôpitaux ou établissements de traitement, devraient mettre en place des procédures et des systèmes auxiliaires permettant de déplacer et de soigner un grand nombre de victimes si les hôpitaux et les établissements de traitement locaux se révèlent incapables de répondre à la demande (par exemple, si la capacité des unités spécialisées est insuffisante, ou si celles-ci sont tout simplement inexistantes).

* Afin de combler les besoins d'urgence, il faudrait prendre des dispositions qui permettent de transformer rapidement des installations normalement utilisées à des fins autres que médicales. Par exemple, lorsque l'accès aux hôpitaux est limité, on peut prévoir l'aménagement provisoire de lieux comme les écoles, les complexes sportifs et des tentes pour offrir des soins médicaux aux victimes de l'accident.

Les plans d'urgence devraient indiquer les mesures de protection à prendre dans l'éventualité où un hôpital ou un établissement de traitement est contaminé ou mis en péril à cause d'un accident (par exemple, coupure de l'approvisionnement en électricité, dommages à la structure, vent soufflant dans une direction propre à entraîner les substances dangereuses vers l'établissement en question).

- Les hôpitaux ou les établissements de traitement devraient prévoir, le cas échéant, des mesures d'évacuation des patients ou de décontamination des lieux.

- Les hôpitaux devraient être préparés à prendre des précautions particulières si des substances dangereuses se retrouvent dans leur périmètre ou s'ils admettent des patients contaminés. 
- 5.c.16 Dans le cadre du processus de planification d'urgence, il faudrait s'assurer de disposer $d^{\prime}$ 'une alimentation en oxygène suffisante, $d$ 'antidotes non périmés et d'autres produits pharmaceutiques nécessaires pour traiter les personnes touchées par les effets des substances dangereuses.

" Il faudrait veiller à préserver l'efficacité des antidotes et autres produits pharmaceutiques gardés en réserve (par exemple, ces produits devraient être entreposés adéquatement et ils ne devraient pas être conservés au-delà de leur date de péremption).

" Les pouvoirs publics et la direction des installations dangereuses devraient encourager l'élaboration d'antidotes efficaces contre celles des substances dangereuses pour lesquelles il n'existe pas d'antidotes du tout ou pas d'antidotes assez efficaces.

- 5.c.17 L'industrie tout comme les pouvoirs publics devraient instituer des mécanismes facilitant la mise en commun et (ou) le partage des ressources médicales en cas d'accident (au sein d'une collectivité donnée, entre des collectivités voisines ou entre des pays). Parmi ces ressources médicales peuvent figurer, par exemple, des installations, de l'équipement, des fournitures, de l'information et du personnel.

- 5.c.18 Les autorités en matière de santé publique et de sensibilisation devraient veiller à ce que tout le personnel médical et paramédical concerné reçoive une formation de base sur les principes de toxicologie médicale et de médecine d'urgence. Cette formation et cette sensibilisation devraient se faire en continu et être renouvelées périodiquement, compte tenu des changements aux plans et dispositions d'urgence, aux risques existant dans les collectivités, aux ressources disponibles et aux autres facteurs pertinents.

- 5.c.19 Il faudrait que des vétérinaires, des biologistes et d'autres spécialistes de la santé du bétail, des animaux de compagnie et des espèces sauvages prennent part à l'organisation et à la planification de l'intervention médicale en cas d'accident, aussi bien pour protéger ces animaux que pour aider leurs propriétaires ou ceux qui en prennent soin. 


\section{ÉTABLISSEMENTS MÉDICAUX : PLANIFICATION POUR LES SITUATIONS D’URGENCE}

Les hôpitaux et autres établissements de traitement devraient se doter de plans d'urgence (coordonnés avec les plans externes locaux). Dans le cadre de la planification pour les urgences, ils devraient :

- maintenir un inventaire de l'équipement disponible pouvant être requis, et avoir en main de l'information à jour sur la façon d'obtenir une aide complémentaire;

- s'assurer de disposer d'équipement et d'installations de décontamination (si ce n'est sur les lieux, du moins par l'intermédiaire d'unités mobiles);

- conserver un répertoire du personnel médical et paramédical qui pourrait être sollicité pour aider les hôpitaux et les autres établissements offrant des soins lors d'une situation d'urgence;

- disposer de plans ou de procédures pour l'acheminement, au besoin, de patients vers d'autres hôpitaux ou établissements (ces plans ou procédures devraient être élaborés en collaboration avec les pouvoirs publics);

- faire installer une ligne téléphonique spéciale (autonome) en service 24 heures sur 24, tous les jours, à l'intention des services d'urgence dans l'éventualité d'un accident, et d'un mode de communication auxiliaire au cas où cette ligne téléphonique ne pourrait être utilisée après un accident;

- avoir accès à de l'information spécialisée et à des spécialistes quant au traitement adéquat des victimes d'exposition;

- instituer des protocoles d'échantillonnage en ce qui concerne le prélèvement, l'entreposage et l'analyse des échantillons biologiques (humains) (ce qui devrait comprendre, aussitôt que possible, l'échantillonnage biologique des personnes exposées ou pouvant avoir été exposées à des substances dangereuses, y compris celles chez qui des symptômes ne se manifestent pas immédiatement);

- disposer de procédures d'enregistrement de tous les patients admis à l'hôpital ou à un autre établissement pour être soignés à la suite d'une exposition à des substances dangereuses;

- disposer de procédures pour protéger les autres patients et le personnel contre la contamination;

- établir des mécanismes de suivi et de contrôle.

5.c.20 Dans le cadre du processus de planification pour les urgences, les pouvoirs publics devraient faire en sorte que des systèmes soient en place pour fournir au public l'information nécessaire après un accident et une fois les mesures d'intervention immédiates prises.

" Lors d'un accident et après celui-ci, il faudrait rapidement communiquer au public des renseignements crédibles, essentiels, fondés, objectifs et précis, et ce, explicitement et de façon continue.

" Ces informations devraient avoir trait aux effets de l'accident à l'extérieur du périmètre des installations, aux risques que d'autres effets nuisibles se produisent hors site, aux mesures à prendre par le public et aux aspects connexes du suivi.

" Pour que l'information fournie au public soit efficace et digne de confiance, elle doit être vraie, même si cela signifie qu'il faut reconnaître sa responsabilité pour une erreur commise.

" La communication sur les risques, lors d'un accident, exige des techniques et des systèmes particuliers, qu'il faut définir. Ceux-ci devraient être décrits dans les documents d'orientation concernant la communication et être inclus dans la formation relative à la planification et à l'intervention applicables aux urgences. 
5.c.21 Dans les cas où un accident lié à une installation dangereuse peut avoir des effets dans les collectivités voisines, la planification et l'intervention applicables aux urgences au sein des diverses collectivités pouvant être touchées devraient être coordonnées. Lorsqu'un accident peut entraîner des effets transfrontaliers, la planification et l'intervention en cas d'urgence devraient faire l'objet d'une coopération entre les pays voisins ${ }^{4}$.

\section{(Voir la section 16.a sur la coopération transfrontalière)}

5.c.22 Les pouvoirs publics devraient établir des procédures pour recouvrer les coûts auprès des responsables des accidents, conformément au principe pollueur-payeur 5 .

5.c.23 Lorsqu'ils sont alertés au sujet d'un accident mettant en cause des substances dangereuses, les autorités chargées de l'intervention devraient déclencher la mise en œuvre de leurs plans d'urgence, y compris les mécanismes prévus pour avertir le public et l'informer des mesures à prendre pour réduire les conséquences néfastes.

\section{d. PUBLIC ET AUTRES PARTIES PRENANTES}

\section{Collectivités et public}

- 5.d.1 Les représentants des collectivités devraient prendre part à l'élaboration des plans d'urgence externes. Ils pourraient également constituer un apport pour les plans d'urgence internes en participant à leur préparation.

5.d.2 Les représentants des collectivités devraient, au besoin, participer au réexamen des plans d'urgence internes et externes pour vérifier s'ils sont adéquats et s'ils correspondent bien aux risques relevés au sein de la collectivité.

5.d.3 Les représentants des collectivités devraient aider l'industrie et les pouvoirs publics à mettre sur pied des programmes de communication sur les risques et à transmettre aux populations pouvant être touchées l'information sur les mesures à prendre en cas d'accident mettant en cause des substances dangereuses pour atténuer les effets nuisibles sur la santé, l'environnement et les biens.

5.d.4 Les représentants des collectivités devraient participer à l'élaboration et à l'exécution des essais ou exercices relatifs aux plans d'urgence externes. Ils devraient également prendre part au processus de débreffage afin de définir les leçons tirées de ces essais et exercices, et ils devraient enfin participer aux activités de suivi connexes.

\section{(Voir l'encadré texte après la section 4.a, sur un « Exemple de représentation de la communauté »)}

\section{Syndicats}

5.d.5 Les syndicats à tous les échelons (local, national et international) ont un rôle important à jouer pour ce qui est de s'assurer que la qualité de la préparation et de l'intervention se situe à un niveau élevé aux installations dangereuses. 
" L'efficacité des programmes de planification pour les urgences repose sur la participation des employés et de leurs représentants, qui doit être aussi importante que possible.

" Une telle participation requiert des syndicats et de la direction qu'ils collaborent au chapitre de l'élaboration, de la mise en œuvre, du contrôle, de la mise à l'essai et de la révision des programmes de planification pour les urgences, de façon à s'assurer que l'information est communiquée efficacement, et à obtenir des employés un degré de compréhension et d'appui optimal vis-à-vis de ces programmes.

" Dans les installations dangereuses où il existe des comités santé/sécurité/environnement réunissant les représentants syndicaux et la direction, il faudrait que ces comités discutent des programmes de planification pour les urgences.

" Les employés et les syndicats peuvent constituer un appui précieux pour véhiculer efficacement l'information jusqu'aux collectivités locales. L'appui des employés et des syndicats en cette matière accroîtra la crédibilité de l'information fournie à ces collectivités.

5.d.6 Les syndicats devraient appuyer les activités de formation et de sensibilisation de leurs membres à la planification pour les urgences (y compris les aspects relatifs à la santé, à la sécurité et à l'environnement).

" Les syndicats sont également responsables de former des représentants en sécurité, qui seront chargés de défendre les intérêts des membres touchant à la santé et à la sécurité. À cet égard, les représentants en sécurité jouent un rôle essentiel au chapitre de la médiation et de la communication entre la direction et les travailleurs.

" L'expérience et les connaissances accumulées par les syndicats grâce à leurs programmes de sensibilisation et de formation et à leurs activités quotidiennes peuvent constituer des sources précieuses de rétro-information au chapitre de la planification et de l'intervention applicables aux urgences.

5.d.7 Les syndicats devraient prendre part à des activités menées en coopération avec la direction à l'échelle locale, nationale et internationale. L'engagement et la participation des syndicats en matière d'élaboration, de promotion, de mise en œuvre, de contrôle et de révision des programmes de planification pour les urgences à l'échelle locale, nationale et internationale constituent, sur une base régulière, des occasions de coopération et de partage de l'expérience, et créent un climat de confiance qui est essentiel.

\section{Établissements de recherche universitaires et autres}




\section{Organisations non gouvernementales (ONG)}

5.d.8 Les ONG devraient contribuer à accroître la connaissance du public en matière de risques et de concepts relatifs aux risques (tant généraux que propres à des installations dangereuses) en participant à la sensibilisation du public quant aux mesures à prendre en cas d'accident et en offrant un soutien humanitaire dans une telle éventualité.

" Les ONG reçoivent souvent des demandes d'information de la part de membres du public qui n'ont pas nécessairement un accès direct et facile à l'information autrement que par elles, ou qui peuvent éprouver des difficultés à bien comprendre l'information et ses implications.

" Les groupes locaux (comme les groupes écologiques) peuvent aider à favoriser la tenue de discussions davantage ciblées entre les représentants des installations dangereuses et les membres des collectivités.

" Les ONG devraient prendre part aux discussions sur l'acceptabilité-tolérabilité des risques, et contribuer à mieux sensibiliser le public à ces risques.

\section{LE PROGRAMME APELL DU PNUE}

Le programme APELL (Awareness and Preparedness for Emergencies at Local Level [Information et préparation au niveau local]) est un outil créé par le PNUE pour réduire au minimum les effets nuisibles des accidents technologiques et des situations d'urgence en accroissant la sensibilisation des collectivités locales et en améliorant la communication entre les parties prenantes. Il définit un processus bien structuré et détaillé pour l'élaboration d'un plan d'intervention d'urgence coordonné, intégré et efficace à l'intention des collectivités locales.

L'APELL est un moyen de rapprocher les parties pour permettre des échanges efficaces sur les risques et l'intervention d'urgence. Cette communication mène à son tour à l'établissement d'un calendrier d'activités.

Le processus de dialogue tel qu'il est recommandé par l'APELL devrait contribuer à

- réduire les risques;

- accroître l'efficacité de l'intervention en cas d'accident;

- permettre aux populations de réagir de manière adéquate aux situations d'urgence.

Pour en savoir plus sur l'APELL, consulter le site

http:/ / www.uneptie.org/pc/apell/home.html (en anglais seulement). 


\section{Chapitre 6}

\section{AMÉNAGEMENT DU TERRITOIRE}

L'élaboration et la mise en œuvre de dispositions relatives à l'aménagement du territoire (zonage et choix des sites) contribuent à la prévention des accidents mettant en cause des substances dangereuses de même qu'à l'atténuation de leurs conséquences. L'aménagement du territoire peut être considéré comme une mesure préventive du fait qu'il aide à séparer les installations dangereuses des autres aménagements par une distance convenable, prévenant ainsi les effets néfastes; il peut aussi être vu comme un moyen d'atténuer les effets nuisibles des rejets, des incendies, des explosions et autres accidents.

Bien que l'aménagement du territoire soit un élément essentiel des stratégies visant à maîtriser les risques associés aux accidents qui mettent en cause des substances dangereuses, il est un complément, et non un substitut, aux autres mesures de prévention et d'atténuation.

Il faut savoir que l'aménagement du territoire, dans le présent contexte, ne concerne pas uniquement le zonage et le choix du site des installations dangereuses, mais également les modifications en profondeur qui sont apportées à des installations existantes. De même, il est primordial d'examiner les questions relatives à l'aménagement du territoire lorsqu'il s'agit de prendre des décisions concernant des aménagements projetés (par exemple, des habitations, des écoles, des boutiques ou autres établissements commerciaux, et des infrastructures publiques telles que des gares ferroviaires) à proximité d'installations dangereuses existantes.

Il faut noter que, dans certains pays, l'aménagement du territoire se décide à l'échelle nationale, en coopération avec les autorités locales, tandis que dans d'autres pays, il s'agit d'une question d'ordre strictement local.

(Étant donné le double rôle tenu par l'aménagement du territoire, des questions connexes sont abordées également à la section $3 b$. Pour embrasser le sujet dans sa totalité, il importe de prendre connaissance des deux ensembles de dispositions.)

6.1 Les pouvoirs publics devraient adopter des dispositions relatives à l'aménagement du territoire de nature à garantir que les nouvelles installations dangereuses sont judicieusement situées du point de vue de la protection de la santé, de l'environnement et des biens, dans l'éventualité d'un accident lié à des substances dangereuses. De plus, les dispositions relatives à l'aménagement du territoire devraient régir les autres aménagements (habitations, commerces, infrastructures publiques) à proximité des installations dangereuses.

" Les dispositions relatives à l'aménagement du territoire ne devraient pas avoir pour effet involontaire d'élever le niveau global de risque pour les personnes susceptibles d'être touchées en cas d'accident.

" À cet égard, les pouvoirs publics devraient prendre en compte les préoccupations $\mathrm{d}^{\prime}$ ordre environnemental et social.

L Lorsqu'ils prennent des décisions concernant le choix du site des installations, les pouvoirs publics devraient tenir compte de tous les risques dans la zone visée afin de limiter autant que possible tout accroissement global du risque pour la santé, l'environnement et les biens. 
6.2 Il faudrait coordonner les activités d'aménagement du territoire des pouvoirs publics locaux, régionaux et nationaux.

" Les pouvoirs publics régionaux et nationaux devraient définir les objectifs généraux à atteindre (en s'appuyant sur les renseignements et les orientations techniques pertinents) pour assurer la cohérence des critères appliqués à l'échelle locale.

" Les autorités locales à l'échelon approprié sont normalement les mieux placées pour prendre les décisions ponctuelles concernant l'aménagement du territoire, en tenant compte des facteurs socio-économiques locaux.

6.3 Les dispositions relatives à l'aménagement du territoire devraient comporter des mécanismes d'application des décisions en matière de zonage et de choix des sites. Les mécanismes assurant l'application et le respect des mesures d'aménagement du territoire prises à des fins de sécurité varieront d'un pays à l'autre en fonction des coutumes, des populations et des systèmes juridiques, mais on peut dégager certains principes généraux.

6.4 Les dispositions et les processus relatifs à l'aménagement du territoire, tout comme les mécanismes de contrôle connexes, devraient indiquer clairement les normes à respecter et les méthodes d'évaluation utilisées par les pouvoirs publics (tant pour les nouvelles installations dangereuses et les modifications aux installations existantes que pour les projets d'aménagements, résidentiels ou autres, à proximité d'installations dangereuses existantes).

" Les décisions concernant l'aménagement du territoire (au chapitre du zonage comme du choix des sites pour les installations dangereuses) tiennent généralement compte des facteurs socio-économiques; il est important que les processus décisionnels soient transparents et que toutes les décisions soient en accord avec l'objectif consistant à atteindre un niveau de sécurité élevé.

" Différentes méthodes peuvent être employées en matière d'aménagement du territoire, dont les suivantes: méthode axée sur les conséquences (repérage des secteurs où des blessures graves seront subies, d'après l'évaluation des répercussions d'un certain nombre de scénarios d'incidents envisageables pour une installation ou un site donnés); méthode axée sur le risque (repérage des secteurs où il existe une probabilité que des dommages de tel ou tel degré se produisent, d'après l'évaluation tant des conséquences que des probabilités associées à divers scénarios d'incidents envisageables pour une installation ou un site donnés); méthode générale (établissement de distances de sécurité en fonction du type d'activités plutôt que d'après une analyse approfondie d'une installation ou d'un site donnés).

Les capacités d'intervention des services d'urgence extérieurs (qui supposent du personnel entraîné et de l'équipement) doivent également entrer en ligne de compte dans l'aménagement du territoire. À cet égard, les dispositions relatives à l'aménagement du territoire ne devraient pas négliger l'importance de choisir - pour l'installation des hôpitaux et des établissements de soins - des secteurs qui ne sont pas susceptibles d'être touchés en cas d'accident lié à des substances dangereuses.

6.6 Si une zone donnée comptant des installations dangereuses n'est pas en mesure de respecter à court terme les lignes directrices en vigueur pour l'aménagement du 
territoire, il convient d'intervenir pour atténuer les risques à long terme, par exemple en modifiant les installations ou en éliminant progressivement les installations anciennes et (ou) les immeubles d'habitation à proximité du site. De telles mesures peuvent toutefois exiger une indemnisation des propriétaires.

- 6.7 Le public devrait se voir offrir l'occasion de participer aux décisions concernant le choix des sites pour les installations dangereuses. Les populations pouvant être touchées devraient être avisées des demandes d'implantation ou de permis soumises en rapport avec des installations dangereuses. Il faudrait aussi que les décisions prises au sujet de ces demandes soient rendues publiques ${ }^{6}$. 


\section{Chapitre 7}

\section{COMMUNICATION AVEC LE PUBLIC}

Ce chapitre aborde la communication d'information au public, considérée comme un élément essentiel de la préparation aux situations d'urgence et de l'atténuation de leurs conséquences (ainsi que des programmes de prévention et d'intervention). Il est entendu, aux termes des présents Principes directeurs, que l'information du public est une responsabilité conjointe des pouvoirs publics et de l'industrie, et que les divers pays et collectivités attribuent différemment les responsabilités relatives à l'information. En outre, les présents Principes directeurs tiennent compte $d u$ fait que les voies de communication doivent fonctionner dans les deux sens, et que les membres des collectivités devraient participer à l'élaboration et à la mise en ouvre des programmes d'information.

7.1 Les membres du public susceptibles d'être touchés en cas d'accident ont le droit d'être informés correctement de façon à connaître les dangers et les risques associés aux installations dangereuses établies dans leur communauté et à pouvoir réagir de manière appropriée en cas d'accident.

7.2 L'information communiquée au public pouvant être touché devrait comprendre des règles d'orientation spécifiques sur ce que l'on peut prévoir en cas d'accident, notamment :

- des détails sur la façon dont le public susceptible d'être touché sera averti si un accident se produit ou menace de se produire de manière imminente;

- des conseils au public pouvant être touché quant aux mesures à prendre et au comportement à adopter en cas d'accident (ces conseils devraient être adaptés en fonction des besoins des différents groupes cibles, y compris les groupes vulnérables se trouvant, par exemple, dans les hôpitaux, les écoles, les foyers pour personnes âgées, etc.);

- des éléments expliquant aux membres du public pourquoi ils devraient se comporter de la manière conseillée, afin qu'ils comprennent en quoi cela permettra d'atténuer les effets néfastes;

- les sources d'information sur les suites de l'accident (par exemple, les radiofréquences et les chaînes de télévision);

- les sources d'explications ou de renseignements supplémentaires;

- les points de contact, où les membres du public peuvent fournir aux pouvoirs publics des renseignements sur un accident éventuel (par exemple, si quelqu'un remarque quelque chose d'inhabituel à l'installation);

- des renseignements sur la façon dont le public sera avisé que tout est rentré dans l'ordre.

- Cette information devrait être fournie en temps opportun, être rediffusée périodiquement au besoin et être mise à jour si nécessaire.

- Il devrait être clairement indiqué qu'il faut prendre immédiatement connaissance de l'information et la conserver dans un endroit où il sera facile de s'y reporter en cas d'accident. 
"Les pouvoirs publics devraient s'assurer que l'information est fournie au public, quoiqu'il incombe à chaque pays ou collectivité de décider qui est responsable de la communiquer ${ }^{7}$.

7.3 Les éléments d'information énumérés au paragraphe 7.2 devraient être transmis au public susceptible d'être touché sans qu'il ait à les réclamer ("information active »), et ces renseignements devraient également être conservés dans un endroit désigné, où le public pourrait les demander.

7.4 Le public pouvant être touché devrait également obtenir sans avoir à les réclamer spécifiquement des renseignements supplémentaires sur les installations dangereuses voisines. Ces renseignements devraient comprendre :

- les types d'industries se trouvant dans le secteur et les produits chimiques qu'elles fabriquent et utilisent (le nom commun ou, si cela convient mieux, le nom générique ou la classe générale de danger des substances présentes à l'installation qui pourraient être à l'origine d'un accident capable de causer des dommages importants hors site, ainsi que les principales caractéristiques nocives de ces substances);

- le nom des entreprises responsables des installations et l'adresse des installations;

- de l'information sur les types d'accidents qui pourraient causer des dommages importants hors site, et sur leurs effets potentiels sur la santé, l'environnement et la propriété;

- les mesures préventives qui ont été prises afin de réduire le plus possible la probabilité d'accidents;

- un renvoi au plan d'urgence externe;

- un ou des points de contact, où le public peut obtenir davantage d'information ou des éclaircissements, et où des renseignements peuvent être fournis aux services de secours et aux divers organismes;

- de l'information sur les activités prévues à l'installation qui risquent d'inquiéter les gens vivant à proximité (par exemple flammes de torchère, odeurs) ${ }^{8}$.

7.5 Il convient de déterminer avec précision quels sont les membres du public pouvant être touchés par un accident, et de cibler la diffusion de l'information pour que toutes ces personnes disposent de renseignements pertinents et suffisants, présentés de manière facilement compréhensible.

" Pour déterminer les groupes auxquels s'adressent ces renseignements, il peut être utile de respecter les limites et regroupements naturels des collectivités afin de ne pas diffuser des informations différentes aux membres d'une même collectivité.

" Il faut savoir que le public n'est pas homogène et, en conséquence, il convient de déterminer s'il est nécessaire d'adapter le message en fonction de différents groupes, basés sur l'âge, le sexe, la culture ou la langue, le niveau de scolarité, le niveau de risque ou d'autres critères.

" Il faudrait tâcher de fournir ces renseignements à toute personne qui emménage ou commence à travailler dans un secteur pouvant être touché en cas d'accident.

" Les renseignements communiqués au public devraient être compréhensibles pour le destinataire moyen ( $c^{\prime}$ est-à-dire les personnes qui ne possèdent pas de connaissances ou de formation techniques) et être présentés sous une forme et (ou) rédigés dans 
une langue facile à lire et à comprendre. Les membres de la collectivité devraient être consultés pour s'assurer que la formulation du message et la langue utilisée sont adaptées à leur groupe.

" Les renseignements concernant les mesures à prendre en cas d'accident doivent être réalistes, sans quoi la crédibilité de l'information sera minée (par exemple, il sera malvenu de recommander à des campeurs de chercher refuge sous leur tente).

" Les renseignements devraient permettre à toutes les personnes concernées de comprendre leurs responsabilités (par exemple, les enseignants ont besoin d'une formation et de renseignements particuliers compte tenu de leurs responsabilités, et ils doivent garantir aux parents que leurs enfants seront bien protégés en cas d'accident).

7.6 Afin d'éviter la confusion et de faciliter l'échange de l'information, les mécanismes d'obtention et de diffusion de l'information devraient être aussi simples que possible et, si possible, utiliser des voies de communication éprouvées.

" Les concepteurs des moyens d'information du public devraient tenir compte de l'expérience acquise en matière de communication des risques dans des situations analogues (par exemple, lors de catastrophes naturelles).

" Les membres de la collectivité devraient être consultés pendant la conception et la mise en œuvre des processus de communication de l'information au public.

7.7 Afin de se les rappeler et de faire en sorte qu'ils parviennent à tous les publics cibles, les messages devraient être répétés périodiquement par l'intermédiaire de différents moyens ou voies de communication.

7.8 Les concepteurs des programmes d'information devraient garder à l'esprit que les messages seront interprétés par les destinataires, filtrés en fonction de l'expérience personnelle et jugés selon le degré de confiance et d'autres facteurs (par exemple, la présence ou l'absence de messages contradictoires). Il est important de comprendre ces influences et de formuler les messages en conséquence.

" Il faudrait se baser, dans la conception des programmes d'information, sur le comportement réel des gens dans une situation d'urgence tel que le révèlent les observations et l'expérience, et non sur ce que les gens affirment qu'ils feraient en cas d'urgence.

"Les réactions prévisibles de la part du public devraient être prises en compte au moment de rédiger des instructions concernant l'intervention en cas d'urgence. À ce chapitre, les réactions du public à des événements affolants et inattendus sont souvent guidées par l'instinct plutôt que par la formation ou les renseignements reçus. Par exemple, les parents voudront spontanément aller chercher leurs enfants à l'école, même si ce geste pourrait aggraver le danger qu'eux-mêmes et leurs enfants courent.

7.9 Les pouvoirs publics, par leur campagne d'information, ne devraient pas se contenter de cibler le public pouvant être touché en cas d'accident; ils devraient aussi sensibiliser la population dans son ensemble aux risques qu'entraînent les accidents mettant en cause 
des substances dangereuses (et aux autres situations d'urgence) ainsi qu'aux mesures qui devraient être prises en cas d'accident. Ceci est essentiel compte tenu de la mobilité aussi bien des gens (ils entrent et sortent des zones à risque) que de certaines sources de risque.

7.10 La responsabilité de communiquer l'information sur les installations dangereuses devrait être confiée à des personnes qui possèdent les connaissances et les compétences appropriées, qui sont perçues comme étant bien informées et dignes de foi, qui inspirent confiance et qui jouissent du respect de la collectivité.

7.11 Les pouvoirs publics, l'industrie et le public devraient avoir l'occasion de se consulter au sujet des divers types d'information à communiquer au public (information active et information fournie sur demande).

"Les pouvoirs publics devraient également organiser des discussions avec le public et les autres parties prenantes au sujet du caractère acceptable/tolérable ou non des risques, de telle façon que le public se familiarise avec les concepts relatifs au risque et puisse aisément participer aux processus décisionnels pertinents.

" Au besoin, les pouvoirs publics devraient envisager la possibilité de mettre sur pied des groupes communautaires à ces fins.

(Voir l'encadré à la suite de la section 4 .a se rapportant à un « Exemple de représentation de la communauté »)

7.12 Les employés devraient contribuer à informer et à la sensibiliser les membres de leur collectivité. Des employés renseignés peuvent jouer un rôle important comme ambassadeurs de la sécurité au sein de la collectivité puisqu'ils ont toutes les raisons de vouloir se protéger eux-mêmes ainsi que leur famille et leur quartier.

7.13 Comme les médias constituent une voie d'information du public en général, il faudrait encourager leur participation à l'élaboration et à la mise en œuvre du processus de communication prévu dans le cadre de la planification en cas d'urgence.

* Il faudrait désigner clairement un ou des médias comme responsables de la communication de l'information en cas d'accident, et les faire connaître au public.

" L'industrie et les pouvoirs publics devraient fournir aux représentants des médias toute l'information pertinente au sujet des installations dangereuses, de telle sorte que ceux-ci aient une idée de la situation générale suffisamment précise pour constituer une source efficace et fiable d'information pour le public en cas d'accident.

" Les médias devraient demeurer une source d'information objective en ce qui concerne les installations dangereuses et les accidents qui s'y produisent; par conséquent, il leur faudrait éviter tout conflit d'intérêts potentiel.

7.14 Les décideurs et autres parties prenantes, y compris le public, devraient obtenir de l'information et de la formation sur les mesures que le personnel d'intervention pourrait prendre en cas d'accident, et ils devraient garder à l'esprit que, pour limiter le plus possible les effets sur la santé, l'environnement et les biens, le mieux est parfois de ne pas intervenir, ou à peine. Les décideurs et le public doivent comprendre pourquoi le personnel d'intervention pourrait limiter ses activités, et ce afin d'éviter que la pression publique ou politique ne pousse à une démarche plus dommageable. 


\section{(Voir paragraphe 10.11)}

7.15 Des procédures devraient être prévues pour associer le public à l'élaboration des plans d'urgence externes'.

(Voir section 5.d sur le rôle du public et des autres parties prenantes dans la préparation et la planification en cas d'urgence)

7.16 Dans les cas où l'installation dangereuse est située près d'une frontière, il devrait y avoir des mécanismes garantissant que l'information soit communiquée à toutes les parties prenantes des deux pays pouvant être touchés en cas d'accident.

\section{(Voir section 16.a sur la coopération transfrontalière)}

7.17 Il faudrait s'employer à faciliter l'échange, entre les collectivités et les pays, des renseignements relatifs aux meilleures pratiques en matière $d^{\prime}$ information du public. 


\section{NOTES}

1. Des travaux visant à mieux comprendre les effets sur la santé de l'exposition aiguë à certains produits chimiques sont en cours dans le cadre de plusieurs projets nationaux et internationaux comme le projet américain de détermination des AEGL (Acute Exposure Guideline Levels; seuils de référence en matière d'exposition aiguë) et le projet ACUTEX de la CE qui, lui, vise à élaborer des méthodes nouvelles pour établir des seuils d'exposition aiguë pouvant être utilisés dans le cadre de la planification pour les situations d'urgence et de l'aménagement du territoire.

2. Il convient de rappeler que le terme «employés » tel qu'on l'entend dans le présent document inclut les contractants et les sous-traitants.

3. Par exemple, l'annuaire international des centres d'urgence (International Directory of Emergency Response Centres; en anglais seulement), publication conjointe de l'OCDE, du PNUE et du BCAH, peut servir de base à l'établissement d'une telle liste.

4. Voir la Convention sur les effets transfrontières des accidents industriels de la CEE-ONU, la Convention sur la protection et l'utilisation des cours d'eau transfrontières et des lacs internationaux de la CEE-ONU de même que la Décision du Conseil de l'OCDE C(88)84(Final).

5. Voir la Recommandation du Conseil de l'OCDE C(89)88(Final).

6. Voir la Décision-Recommandation du Conseil de l'OCDE C(88)85(Final).

7. Voir la Décision-Recommandation du Conseil de l'OCDE C(88)85(Final).

8. Voir la Décision-Recommandation du Conseil de l'OCDE C(88)85(Final).

9. Voir la Décision-Recommandation du Conseil de l'OCDE C(88)85(Final). 


\section{Partie C}

\section{INTERVENTION D'URGENCE}

Cette partie traite des rôles et responsabilités de l'industrie, des pouvoirs publics, du public et des autres parties quant à l'intervention en cas d'accident chimique. Elle est considérablement plus courte que la partie B (Préparation aux situations d'urgence et atténuation de leurs conséquences) puisque les mesures d'intervention devraient essentiellement consister à appliquer les plans d'urgence, en tenant pour acquis que le processus de planification préalable était complet et efficace. (Voir le chapitre 5 sur la préparation et la planification en cas d'urgence.)

Les mesures décrites dans la présente partie se situent à l'échelle locale: elles concernent les lieux de l'accident. Par conséquent, les pouvoirs publics visés ici comprennent les autorités locales d'intervention (c'est-à-dire les pompiers, les secours médicaux d'urgence et la police), les organismes gouvernementaux locaux ou régionaux ainsi que les établissements sanitaires et médicaux. 


\section{Chapitre 8}

\section{PRINCIPES GÉNÉRAUX}

8.1 On devrait disposer de systèmes permettant d'alerter immédiatement le personnel d'intervention en cas d'accident lié à des substances dangereuses ou de menace imminente d'un tel accident nécessitant son entrée en action.

" La mise en alerte du personnel d'intervention devrait déclencher la mise en oeuvre du plan d'urgence.

" Il faudrait alors activer les systèmes permettant d'alerter le public susceptible d'être touché par l'accident.

8.2 Les parties responsables de l'intervention d'urgence devraient être associées au processus de planification. Après l'intervention, le plan d'urgence devrait être réexaminé et modifié, au besoin, à la lumière de l'expérience acquise.

8.3 En cas d'accident lié à des substances dangereuses, les parties prenantes devraient prendre toutes les mesures raisonnablement envisageables pour réduire le plus possible l'exposition des gens à ces substances et pour limiter les effets néfastes sur la santé, l'environnement et les biens.

8.4 Les porte-parole désignés pour fournir l'information au public à la suite d'un accident (y compris les représentants de l'industrie et des pouvoirs publics) devraient posséder les connaissances, les compétences, l'autorité et la crédibilité nécessaires pour communiquer efficacement avec le public.

- Les porte-parole officiels devraient adopter une attitude aussi ouverte que possible lorsqu'ils communiquent des renseignements lors d'un accident et après.

n Dans cette optique, ils devraient, par exemple, présenter l'information en temps opportun, admettre, le cas échéant, qu'ils ne disposent pas de certaines informations, éviter de faire des promesses impossibles à tenir, être les premiers à annoncer les mauvaises nouvelles et faire en sorte que les messages transmis correspondent aux mesures adoptées. 


\section{Chapitre 9}

\section{INDUSTRIE}

- En cas d'accident lié à des substances dangereuses, la direction des installations en cause devrait immédiatement mettre en œuvre son plan d'urgence interne.

9.2 S'il survient un accident lié à des substances dangereuses qui entraîne ou menace d'entraîner des dommages à la santé, à l'environnement ou aux biens hors site, ou encore un accident que les ressources d'intervention internes sont impuissantes à maîtriser, la direction ou les employés des installations dangereuses devraient sans tarder alerter les autorités locales responsables des interventions d'urgence.

NOTE : Cela est différent de l'obligation qu'a l'industrie d'aviser les pouvoirs publics après un accident, à des fins de tenue de dossier ou d'application de la loi (voir chapitre 14).

- Une fois alertés, les pouvoirs publics devraient déclencher la mise en œuvre du plan d'urgence externe, en commençant par une évaluation préliminaire de la situation pour arrêter les décisions quant aux mesures à prendre.

* La direction devrait être préparée à aider le personnel d'intervention en lui fournissant de l'information, de l'expertise et d'autres ressources, au besoin.

9.3 Les plans d'urgence devraient comprendre des critères qui établissent nettement quand les pouvoirs publics devraient être appelés en renfort pour répondre à un accident pouvant avoir des effets hors site.

* Ces critères devraient fixer à quel stade précisément il convient de contacter les pouvoirs publics, et qui appeler.

- Même si les pouvoirs publics participent à l'intervention, l'entreprise demeure responsable de la sécurité de ses installations, y compris des effets nuisibles causés par tout accident. 


\section{Chapitre 10}

\section{POUVOIRS PUBLICS}

10.1 Lorsqu'elles sont averties qu'un accident lié à des substances dangereuses s'est produit, les autorités chargées de l'intervention devraient déclencher la mise en œuvre de leur plan d'urgence.

" L'objectif est de réunir les moyens nécessaires pour déterminer le lieu de l'accident et, si possible, pour le contenir et, ainsi, atténuer les effets nuisibles sur la santé, l'environnement et les biens.

"Le plan d'urgence devrait prévoir des mécanismes pour s'assurer que le public est au courant de l'accident et informé des mesures à prendre pour en limiter les conséquences nuisibles.

10.2 Le coordonnateur sur place devrait prendre les décisions quant aux mesures à prendre dans l'immédiat afin d'éviter ou de limiter l'exposition humaine aux substances dangereuses, tant sur le site qu'à l'extérieur.

" Cela devrait inclure des mesures visant à empêcher la contamination des secouristes.

" Le coordonnateur sur place devrait être préparé à obtenir de l'information et des conseils de la part de la direction de l'entreprise, de même que d'autres spécialistes, sur les meilleurs moyens de protéger la santé, l'environnement et les biens contre les substances dangereuses en cause dans l'accident.

" Le personnel médical et paramédical de même que l'équipe d'intervention devraient prêter main-forte au coordonnateur sur place, à la demande de celui-ci, pour les mesures à prendre dans l'immédiat pour éviter ou limiter l'exposition des employés, des secouristes et du public.

- 10.3 Dans les cas où il y a déversement d'une substance toxique, c'est le coordonnateur sur place ou les autres personnes désignées à cet effet dans le plan d'urgence qui devraient décider si le public susceptible d'être touché doit s'abriter à l'intérieur ou être évacué. La décision devrait être fondée sur la probabilité d'exposition et les effets potentiels sur la santé.

10.4 Étant entendu que les décisions relatives aux mesures immédiates sont généralement motivées d'abord et avant tout par la nécessité de protéger les personnes contre les effets toxiques aigus, les décisions concernant l'intervention devraient également prendre en compte les effets potentiels à long terme et les effets tardifs sur la santé causés par l'exposition (directe et indirecte) aux substances dangereuses, ainsi que les répercussions possibles sur l'environnement.

" Le personnel d'intervention (y compris le personnel médical et paramédical) devrait savoir que les personnes exposées à des substances dangereuses peuvent subir des effets nuisibles sans que cela se traduise par des symptômes immédiats et évidents. Par conséquent, les personnes exposées devraient être placées en observation et, s'il y 
a lieu, recevoir tout de suite des soins et être transportées vers un établissement de traitement.

4. Il faudrait disposer de renseignements permettant d'étayer les décisions concernant la façon de traiter les personnes exposées aux produits chimiques, qui pourraient subir des effets nuisibles à long terme, tardifs ou non décelés.

\section{(Voir le chapitre 13 sur les aspects médicaux du suivi des incidents)}

10.5 Il faudrait prendre des dispositions pour que les premiers secours et autres soins médicaux soient prodigués, si possible, à l'extérieur de la zone contaminée.

" L'accès aux zones contaminées devrait être strictement contrôlé; seul le personnel d'intervention requis devrait être autorisé sur les lieux.

" De manière générale, les premiers soins devraient être administrés près des lieux de l'accident, mais à l'extérieur du rayon de contamination, de sorte que l'état des blessés soit stabilisé avant leur transport (s'il y a lieu) vers un établissement de soins.

" Il importe d'abord et avant tout de soustraire la personne à l'exposition aux substances dangereuses. Ensuite, on peut s'occuper des effets physiologiques et psychologiques.

" En plus des premiers soins habituels, il peut s'avérer nécessaire d'entreprendre d'autres traitements sur les lieux de l'accident. En conséquence, des fournitures et du matériel spécialisés, y compris des antidotes, devraient, le cas échéant, être disponibles sur place.

10.6 Pour trier les victimes des accidents mettant en cause des substances dangereuses, le personnel d'intervention (et autres personnels d'intervention d'urgence, selon le cas) devraient appliquer les mêmes règles que celles qui sont adoptées généralement dans les situations d'urgence. Les personnes appartenant aux groupes vulnérables (comme les enfants) devraient normalement être traitées en priorité.

10.7 Des systèmes devraient être prévus pour réunir le matériel, les spécialistes et les autres ressources nécessaires à l'intervention; il faudrait notamment être en mesure d'obtenir s'il y a lieu de l'aide de la part des autorités régionales et nationales ou du personnel pour les interventions d'urgence des collectivités voisines ou autres, selon les besoins.

10.8 Le premier personnel d'intervention arrivé sur les lieux d'un accident devrait posséder des renseignements, une formation et une expérience suffisants pour évaluer rapidement s'ils peuvent faire face à la situation ou s'ils doivent demander des renforts (matériel ou personnes possédant des compétences particulières).

" Il faudrait disposer de systèmes donnant un accès immédiat, sur les lieux mêmes, aux renseignements qui peuvent être utilisés pour évaluer la situation d'urgence et y répondre. De plus, il faudrait prévoir des systèmes permettant de recueillir, de diffuser et de mettre à jour l'information destinée à être communiquée au personnel médical et paramédical ainsi qu'aux autres parties concernées au fur et à mesure que l'intervention d'urgence progresse, y compris les renseignements ou avis médicaux émis à l'intention du public par les médias. 
" Il faudrait disposer de lignes directrices en ce qui concerne le choix de la stratégie d'intervention en cas d'accident, sachant qu'il pourrait être nécessaire de faire ce choix même si l'on dispose de peu de temps et d'informations incomplètes.

10.9 Les renseignements utilisés à l'appui des mesures d'intervention, qui devraient être mis à jour et diffusés régulièrement, comprennent les éléments énumérés ci-dessous.

"Les données sur la quantité et la nature des substances dangereuses mises en cause, notamment les suivantes : propriétés physico-chimiques; transformation possible des produits de décomposition des substances, par exemple au contact de l'eau ou par pyrolyse; propriétés toxicologiques et écotoxicologiques; effets cliniques, y compris les effets aigus, tardifs et à long terme; évaluations des risques.

" Des lignes directrices concernant le seuil d'exposition aiguë à diverses substances, les effets nocifs probables et les moyens de protection contre ces effets.

"Des renseignements sur le nombre et le type de patients prévisibles, sur la nature de leurs blessures et sur la gravité de leur exposition.

"La nature de l'information sur les premiers soins et les traitements médicaux devrait être adaptée au public cible qui comprend, notamment, le profane (sans formation médicale), le médecin généraliste et le personnel médical spécialisé (par exemple, le spécialiste en soins intensifs). Cette information devrait couvrir les points suivants :

- les signes, les symptômes et le moment prévisible d'apparition des symptômes après l'exposition selon diverses voies (exposition oculaire, inhalation, absorption cutanée, ingestion);

- les moyens de décontamination des victimes;

- la façon de trier et d'enregistrer un nombre potentiellement grand de victimes (compte tenu des circonstances locales);

- les soins médicaux (y compris l'administration d'antidotes, s'il y a lieu), selon les circonstances, la gravité de l'état des victimes et l'accès à des hôpitaux ou à d'autres établissements de soins;

- le moment où l'on peut s'attendre à ce que des symptômes se déclarent chez des personnes au départ asymptomatiques (au bout de quelques heures ou davantage);

- la façon de recueillir et d'entreposer des échantillons à des fins d'analyse de la toxicité et d'autres essais;

- les mesures de protection que le personnel médical et l'équipe d'intervention d'urgence devraient prendre pour éviter d'être contaminés;

- l'endroit où se trouvent les fournitures pharmaceutiques nécessaires;

- l'emplacement des laboratoires et le type d'analyses qu'ils peuvent effectuer.

" Des renseignements au sujet des ressources et des installations disponibles, notamment :

- les établissements médicaux (c'est-à-dire l'emplacement des centres de soins de santé, des hôpitaux et des dispensaires, de même que leur capacité, par exemple, en lits, en ventilation mécanique, en alimentation en oxygène et en matériel spécialisé);

- les fournitures pharmaceutiques;

- les installations de décontamination;

- les renforts en personnel médical, y compris en spécialistes;

- les services de biosurveillance; les installations de laboratoire; 
- les sources d'information.

Les moyens de transport pour les victimes (ambulances, hélicoptères).

"Quand et comment contacter les services essentiels, dont les autorités centrales, les autorités locales, la police, les pompiers et les autres services de secours.

-Qui est le coordonnateur local en cas d'urgence, et quels sont les critères qui justifient le transfert de l'autorité et de la supervision à une autorité supérieure.

- La liste des spécialistes (au sein de l'industrie, des pouvoirs publics, etc.) capables de donner des avis sur des substances dangereuses ou des groupes de substances dangereuses en particulier.

" Les renseignements ou avis médicaux communiqués au public par les médias.

10.10 Dans les cas où ce qu'exige l'intervention dépasse les prévisions établies dans le plan d'urgence, ou que des difficultés d'un autre ordre se présentent (par exemple, la sécurité du personnel d'intervention est menacée), le coordonnateur sur place devrait demander de l'aide.

- Cette aide peut revêtir la forme de renseignements communiqués par des spécialistes de l'extérieur (dans le domaine, par exemple, de la toxicologie, du génie chimique, de la médecine, etc.) aptes à donner rapidement un avis fiable. Ces renseignements devraient être présentés de manière à pouvoir être compris et exploités par le personnel d'intervention. Le coordonnateur peut, par exemple, avoir besoin des types d'information suivants :

- $\quad$ substances dangereuses en cause;

- $\quad$ suggestions quant aux mesures à prendre;

- évaluation des risques;

- besoins en équipement de protection;

- endiguement et confinement des substances dangereuses;

- décontamination et activités pour mettre fin à la situation d'urgence.

" Si un accident ne peut être maîtrisé grâce aux ressources locales d'intervention (c'està-dire l'équipement, le matériel et le personnel), il faudrait lancer des appels pour obtenir de l'aide des collectivités voisines, des autorités régionales ou nationales et (ou) d'autres pays.

10.11 Malgré l'inclination naturelle ou la pression qui poussent à intervenir de façon proactive pour répondre à un accident chimique, le personnel d'intervention d'urgence devrait être conscient $\mathrm{du}$ fait qu'à certains stades de l'intervention, il vaut mieux s'abstenir d'intervenir ou de limiter cette intervention afin de réduire autant que possible les effets nuisibles sur la santé, l'environnement ou les biens.

" Tout type d'intervention (y compris les mesures de faible envergure) requiert un contrôle et un suivi appropriés pour s'assurer que toutes les conséquences sont bien comprises et que les mesures visant à protéger la santé et l'environnement sont prises là où il y a lieu de le faire. 
" Il importe que les décisions concernant les mesures appropriées à prendre puissent être modifiées au cours du temps, d'après l'évolution de la situation et l'examen des renseignements mis à jour.

- 10.12 Les hôpitaux et autres établissements de soins devraient mettre en œuvre leurs plans d'urgence ainsi que la partie du plan d'urgence communautaire placée sous leur responsabilité dès qu'ils sont avisés que des patients victimes d'un accident lié à des substances dangereuses pourraient leur être acheminés.

10.13 Au cours d'une intervention d'urgence, le personnel médical et paramédical ainsi que les établissements de santé devraient faire partie de l'équipe globale d'intervention et du circuit d'information, de manière à pouvoir fournir et recevoir des renseignements au besoin.

10.14 Les hôpitaux et autres établissements de santé susceptibles de participer à l'intervention liée à un accident devraient recevoir, dès que possible, des renseignements sur les substances dangereuses en cause, le type d'accident survenu (déversement, incendie, etc.), le nombre probable de victimes et la nature de leurs blessures.

" Ces renseignements devraient être utilisés pour faire une évaluation préliminaire des effets possibles sur la santé humaine et du meilleur traitement à prodiguer.

" Il faudrait disposer de protocoles thérapeutiques et, dans la plupart des cas, les respecter, surtout si les victimes de l'accident sont acheminées vers plusieurs établissements de santé (étant entendu, toutefois, qu'une certaine latitude est nécessaire pour tenir compte de la vulnérabilité variable d'un individu à l'autre et $\mathrm{d}^{\prime}$ autres facteurs pertinents) ${ }^{1}$.

\section{(Voir l'encadré au chapitre 5 sur la planification en cas d'urgence dans les établissements médicaux)}

- 10.15 Afin de traiter de manière appropriée les personnes exposées, le personnel médical et paramédical devrait avoir accès à de l'information spécialisée et consulter, si besoin est, différents spécialistes (par exemple, des toxicologues, des pneumologues et d'autres spécialistes du système respiratoire, des ophtalmologistes, des hématologues et des médecins du travail).

10.16 Après un accident, il faudrait très vite offrir un soutien psychologique aux personnes qui en ont besoin. Concrètement, il faudrait que des professionnels ou des conseillers possédant une formation en psychiatrie, en psychologie ou en intervention psychosociale soient rapidement à l'œuvre sur place.

" Ces professionnels ou conseillers peuvent offrir un soutien affectif aux victimes et à leurs proches ainsi qu'au personnel d'intervention; collaborer avec les services d'information; participer à la détection des éventuels problèmes de santé mentale; contribuer à l'établissement de réseaux de suivi destinés à dépister et à traiter les séquelles psychologiques.

" Il faudrait tenir compte, dans le processus de planification, du rôle d'autres personnes pouvant apporter un soutien affectif et psychologique (par exemple, les membres du clergé, les entrepreneurs de pompes funèbres), surtout dans les collectivités où le nombre de psychologues et de psychiatres n'est pas suffisant. 
4Dans les zones à haut risque, il faudrait disposer de données épidémiologiques et de moyens reconnus à l'échelle internationale pour évaluer les effets sur la santé mentale, de sorte qu'un suivi puisse être effectué après un accident.

10.17 Lorsqu'un accident entraîne des décès, toutes les personnes qui entrent en contact avec les dépouilles (le personnel d'intervention, les médecins légistes, les embaumeurs) devraient se protéger contre toute contamination éventuelle par les substances dangereuses. Il faudrait qu'une personne responsable de fournir information et assistance aux personnes qui entrent en contact avec des dépouilles contaminées soit désignée dans le plan d'urgence.

10.18 Les pouvoirs publics régionaux ou nationaux devraient autant que possible appuyer les mesures d'intervention locales afin de contribuer à la protection de la santé, de l'environnement et des biens. Ce soutien pourrait consister (sans exclure d'autres possibilités) à :

- fournir des renseignements et des avis techniques, scientifiques, politiques, météorologiques et juridiques au personnel d'intervention;

- entreprendre des inspections et le prélèvement d'échantillons pour déterminer l'ampleur de la contamination;

- déterminer quelles ressources naturelles, entre autres animales, sont menacées, prévoir le devenir du déversement, faire des prévisions météorologiques et établir les priorités en matière de protection;

- fournir des éléments d'orientation pour la protection et le rétablissement des espèces sauvages.

10.19 Le personnel d'intervention (et d'autres intervenants) devrait étayer les décisions et les mesures prises au cours de l'intervention suite à un accident (par exemple, les alertes, les décisions relatives à l'évacuation ou à l'utilisation d'abris, etc.) de manière à pouvoir réexaminer l'efficacité des opérations, apprendre de l'expérience vécue, améliorer les plans d'urgence, alimenter l'enquête et tirer des leçons dans l'éventualité de futures interventions. L'expérience devrait, le cas échéant, être partagée avec les autres entreprises ou collectivités.

10.20 Au cours de la transition entre les activités d'intervention ou de secours et les opérations de remise en état, tous les intervenants devraient coopérer et échanger les informations afin de maintenir la sécurité sur les lieux et de protéger et (ou) de restaurer l'environnement et la propriété.

10.21 Les pouvoirs publics devraient, le cas échéant, appliquer le principe pollueur-payeur et les procédures connexes pour recouvrer les coûts auprès des responsables de l'accident ${ }^{2}$. 


\section{Chapitre 11}

\section{PUBLIC ET AUTRES PARTIES PRENANTES}

\section{a. PUBLIC ET COLLECTIVITÉS}

11.a.1 Les membres du public pouvant être touchés devraient connaître les systèmes d'alerte utilisés en cas d'accident mettant en cause des substances dangereuses. Après une alerte, ils devraient suivre les instructions reçues dans le cadre du processus de planification ainsi que toutes les indications supplémentaires fournies par les voies de communication désignées à cet effet.

11.a.2 À la suite d'un accident et au cours des phases initiales de l'intervention d'urgence, les membres du public devraient pouvoir compter sur les pouvoirs publics compétents pour leur fournir de l'information, en se servant de systèmes éprouvés, facilement accessibles et compréhensibles.

11.a.3 Les membres du public devraient aviser un représentant officiel compétent lorsqu'ils remarquent quelque chose d'inhabituel à une installation dangereuse.

\section{b. MÉDIAS}

- 11.b.1 Les médias devraient pouvoir compter sur un accès facile et permanent à des représentants officiels désignés possédant l'information pertinente ainsi qu'à d'autres sources; ainsi, ils pourront fournir au public des renseignements essentiels et exacts tant que dure la situation d'urgence, et aideront à éviter la confusion.

" Il faudrait s'employer à vérifier la clarté et la fiabilité des renseignements lorsqu'ils sont communiqués aux médias, avant leur diffusion dans le public.

" Les autorités en matière de santé publique devraient être consultées lorsque des déclarations concernant les aspects sanitaires des accidents chimiques sont faites aux médias.

11.b.2 Les médias devraient, afin de réduire les effets nuisibles sur la santé, l'environnement et les biens, faciliter les efforts d'intervention en communiquant au public des informations dignes de foi à propos des accidents mettant en cause des substances dangereuses, y compris des avis sur les mesures à prendre par les personnes susceptibles d'être touchées. Les médias ne devraient pas entraver le déroulement de l'intervention d'urgence (par exemple, en cherchant à pénétrer dans les zones d'accès restreint).

\section{c. ORGANISATIONS NON GOUVERNEMENTALES (ONG)}

11.c.1 Les ONG pourraient constituer une source précieuse d'expertise et d'information dans le cadre d'une intervention d'urgence. Les membres des ONG pourraient aider le personnel d'intervention en accomplissant certaines tâches précises, prévues dans le cadre du processus de planification pour les situations d'urgence. Il pourrait s'agir notamment 
d'offrir un soutien humanitaire, psychologique et social aux membres de la collectivité et au personnel d'intervention (par exemple, la Croix-Rouge), de l'aide pour l'évaluation et l'atténuation des effets des accidents sur l'environnement, et un soutien pour la protection des animaux. 


\section{NOTES}

1. Le Programme international sur la sécurité des substances chimiques a élaboré des protocoles et des traitements de référence.

2. Voir la Recommandation du Conseil de l'OCDE C(89)88(Final). 


\section{Partie D}

\section{SUIVI DES INCIDENTS (Accidents et quasi-accidents)}

\footnotetext{
Dans cette partie, on traite des mesures à prendre après qu'un accident ou un quasi-accident s'est produit, après la phase initiale immédiate d'intervention. La préoccupation essentielle est de tirer des leçons de l'expérience, de manière à éviter que des incidents semblables surviennent dans le futur; par conséquent, la partie D porte sur l'évaluation des conséquences, la déclaration des incidents et les enquêtes.

On n'aborde pas, dans le présent document, les questions relatives à la remise en état consécutive à un accident, par exemple la dépollution de l'environnement. Cependant, on reconnaît qu'effectivement, les mesures prises pendant la phase d'intervention peuvent avoir une incidence sur les opérations de remise en état. C'est pourquoi le travail d'intervention devrait être mené de manière à obtenir les données et les infrastructures nécessaires à la remise en état.
} 


\section{Chapitre 12}

\section{ESTIMATION DES CONSÉQUENCES}

\section{(Voir aussi le chapitre 13 traitant des aspects médicaux du suivi)}

12.1 Il faudrait procéder à l'estimation des conséquences des accidents chimiques (y compris les répercussions sur l'environnement) aussi rapidement que possible après l'accident.

" Il faudrait établir des protocoles définissant les types de données à relever, la manière d'entreprendre les évaluations appropriées (compte tenu des différences entre les démarches privilégiées dans chaque pays).

" Les personnes participant aux opérations sur le terrain devraient avoir reçu la formation nécessaire pour, par exemple, être en mesure de prélever des échantillons et de comprendre les éventuelles répercussions sur l'environnement.

" Il faudrait accélérer l'utilisation des nouvelles technologies, comme les systèmes par satellite et autres systèmes de détection à distance, pour déterminer les zones contaminées et pour améliorer la rapidité des alertes dans les zones où il existe un risque significatif de dommages à l'environnement suite à un possible accident chimique.

" Le principe pollueur-payeur devrait être appliqué, s'il y a lieu, de manière à obtenir les ressources humaines et autres qui sont requises pour l'estimation des conséquences des accidents sur l'environnement. L'application de ce principe peut également inciter fortement la direction des installations dangereuses à prendre tous les moyens à sa disposition pour éviter de tels accidents ${ }^{1}$.

12.2 Il faudrait chercher à mieux comprendre les conséquences des accidents sur l'environnement.

" Afin de pouvoir estimer les conséquences des accidents sur l'environnement, la préparation devrait comprendre l'acquisition de données géographiques et géologiques de base sur les zones entourant les installations dangereuses. De plus, une surveillance devrait être exercée dans le cas des secteurs particulièrement préoccupants du point de vue de l'environnement.

Les parties concernées devraient rassembler les données concernant les effets environnementaux nuisibles causés par les accidents graves, et publier les résultats de leurs recherches.

D Les zones touchées par des accidents liés à des substances dangereuses devraient être surveillées pour déterminer si l'exposition aiguë à ces substances entraîne des effets à long terme ou tardifs.

D Le partage de l'information au sujet des conséquences des accidents sur l'environnement devrait être élargi et, à l'échelle internationale, il faudrait s'employer à réexaminer et à évaluer conjointement ces données. Dans cette perspective, il faudrait faire en sorte que les données environnementales relatives 
aux accidents survenus dans le passé soient compilées dans des bases, comme celles gérées par le MAHB (Major Accident Hazards Bureau) de la CE, au nom de la CE, de l'OCDE et de la CEE-ONU. 


\section{Chapitre 13}

\section{ASPECTS MÉDICAUX DU SUIVI}

13.1 Il faudrait mettre en place des procédures de suivi épidémiologique et médical appropriées pour contrôler et continuer d'observer les personnes ayant subi une exposition à des substances dangereuses, y compris celles qui en apparence n'ont pas souffert de cette exposition. L'apparition des symptômes peut se produire à retardement, des heures ou des jours après l'exposition. L'examen immédiat aidera ultérieurement au diagnostic et au traitement des symptômes.

" Les pouvoirs publics devraient veiller à ce que les personnes ayant subi une forte exposition à des produits chimiques toxiques lors d'un accident soient convenablement examinées et enregistrées en vue d'un suivi à court et à long termes, qu'elles semblent ou non souffrir de cette exposition. Ce processus d'enregistrement devrait faire partie intégrante de l'intervention.

"Il faudrait effectuer des prélèvements biologiques sur les personnes exposées (ou susceptibles d'avoir été exposées) dans un délai aussi court que possible après l'exposition et, s'il y a lieu, à intervalles réguliers par la suite.

" Il faudrait respecter une méthode systématique de contrôle et d'échantillonnage afin de garantir la qualité de la cueillette et de l'analyse des données, et de permettre des comparaisons dans le temps et entre différents événements.

" Il peut s'avérer nécessaire de rechercher les personnes susceptibles d'avoir été exposées pour les soumettre à l'observation et aux traitements nécessaires.

4Il faudrait consigner les mesures de suivi de manière appropriée.

13.2 Après un accident grave, il faudrait établir et mettre en œuvre des protocoles convenables d'épidémiologie et de prélèvement, et ce dans le but de définir l'accident, d'aider à en limiter les conséquences nuisibles sur la santé et l'environnement, et d'apprendre de l'expérience vécue. Afin d'améliorer la valeur de la surveillance et de l'échantillonnage, il faudrait disposer de données sur l'environnement et les populations à proximité des lieux de l'accident et avant ce dernier (pour connaître, par exemple, le degré normal d'exposition et la nature des écosystèmes locaux).

13.3 Il faudrait s'employer davantage à partager l'information au sujet des effets à long terme et à court terme de l'exposition aiguë à des substances dangereuses sur la santé2.

13.4 Il faudrait encourager davantage le partage de l'information entre les spécialistes en santé (y compris les chercheurs) au sujet des traitements appropriés et du suivi épidémiologique et médical à appliquer aux accidents mettant en cause des substances dangereuses ${ }^{3}$. 


\section{CONSTITUTION DE DOSSIERS SUR LES INCIDENTS ET DÉCLARATIONS}

\section{(Déclaration des accidents et quasi-accidents antérieurs)}

\section{a. PRINCIPES GÉNÉRAUX}

14.a.1 La direction des installations dangereuses de même que les associations industrielles, les pouvoirs publics et les autres parties prenantes devraient réfléchir sur la manière de créer un climat propice à la confiance et au partage volontaire de l'information concernant les accidents et les quasi-accidents, y compris les leçons apprises de ceux-ci.

- Il convient de développer davantage et de promouvoir des mécanismes qui favorisent un échange ouvert et franc d'informations concernant les accidents et les quasi-accidents, tant à l'intérieur d'une entreprise donnée qu'entre les entreprises. Il existe un besoin évident de relever et de partager largement ces renseignements dans toute l'industrie, de telle façon que les entreprises puissent profiter de l'expérience des autres.

" En plus de partager les renseignements au sein de l'industrie, il convient d'élaborer des moyens de faire participer les pouvoirs publics à ces échanges, sans compromettre les intérêts des entreprises.

\section{b. POUVOIRS PUBLICS}

14.b.1 Les pouvoirs publics devraient exiger que les éléments clés des accidents liés à des substances dangereuses soient rapidement notifiés ou déclarés (à une autorité compétente). Cette notification devrait être suivie de rapports écrits officiels fournissant des renseignements complémentaires au sujet de l'accident en question.

14.b.2 Les pouvoirs publics devraient définir des critères, des exigences et des procédures appropriés pour la constitution de dossiers sur tous les incidents graves. Cela comprend tant la cueillette d'informations par le personnel d'intervention d'urgence que la compilation de renseignements par la direction de l'installation où l'accident s'est produit.

- La constitution efficace d'un dossier par l'industrie et les pouvoirs publics peut contribuer grandement à l'exploitation des installations dangereuses en toute sécurité. Elle aide aussi à cultiver chez le public la confiance que le nécessaire est fait pour éviter que des incidents semblables ou des incidents aux conséquences analogues se reproduisent.

- Il faudrait constituer un dossier non seulement sur les accidents graves, mais aussi sur les quasi-accidents importants.

" Il faudrait que le dossier ainsi constitué puisse servir de base pour déterminer quels incidents devraient faire l'objet d'une enquête, contribuer à mettre en lumière les tendances de même que les zones préoccupantes, établir les causes et les 
conséquences des incidents, permettre de tirer des leçons de l'expérience, et ouvrir la voie à des mesures correctives propres à combler toute lacune dans la technologie ou les procédures ayant conduit à l'incident.

" Les rapports sur les accidents passés que soumet l'industrie aux autorités devraient inclure des renseignements sur les conséquences des accidents pour l'environnement et la santé. Les répercussions économiques des accidents devraient également être évaluées dans la mesure où le permet l'existence de données pertinentes (les répercussions économiques recevant ici une définition très large, qui englobe notamment aussi bien les coûts directs que les coûts indirects).

" Les pouvoirs publics devraient inciter les entreprises à leur signaler spontanément, au-delà de ce qui est légalement requis, les quasi-accidents graves et les accidents.

14.b.3 Les pouvoirs publics devraient mettre en place un système national structuré pour tenir des statistiques et compiler l'information sur les accidents liés à des substances dangereuses.

" Un tel système améliorera les processus décisionnels, fournira des indications qui permettront d'améliorer la réglementation et les lignes d'orientation, aidera à établir les priorités, contribuera à la préparation des analyses et facilitera l'échange ainsi que la diffusion de l'information et des leçons apprises.

" Les pouvoirs publics et l'industrie devraient s'employer à améliorer l'échange, à l'échelle internationale, des renseignements sur les accidents et les quasi-accidents importants liés à des substances dangereuses. Les statistiques et l'information concernant de tels accidents et quasi-accidents, figurant dans les systèmes nationaux, devraient être versées dans les systèmes internationaux de manière à promouvoir la sécurité, à profiter de l'expérience des autres, et à fournir des données suffisantes pour effectuer des analyses et tenir des statistiques ${ }^{4}$.

\section{c. INDUSTRIE}

14.c.1 La direction devrait respecter toutes les procédures établies pour la notification ou la déclaration des incidents aux pouvoirs publics.

14.c.2 Tous les décès, tous les incidents importants (c'est-à-dire les accidents et les quasiaccidents) et tous les autres événements justifiant une déclaration selon les critères de l'entreprise devraient être déclarés par la direction locale d'une installation dangereuse aux membres compétents de la direction de l'entreprise.

14.c.3 L'entreprise, au nom de la culture de la sécurité, de même que toutes les parties concernées devraient promouvoir la déclaration des accidents et des quasi-accidents aux gestionnaires compétents au sein de l'entreprise, de façon qu'il soit possible d'en déterminer les causes.

" Il faudrait disposer de procédures de déclaration des incidents.

" Les employés devraient recevoir une formation appropriée sur l'identification des dangers, de manière à faciliter la déclaration des incidents. 
4 Il faudrait compter parmi les événements justifiant une déclaration ceux qui surviennent pendant des travaux menés en collaboration avec des contractants.

"Il faudrait s'efforcer de susciter un climat tel que la déclaration des incidents et les débats à leur propos soient considérés comme des activités constructives.

" Les employés devraient aussi être encouragés à discuter des quasi-accidents entre eux, avec leurs représentants et avec la direction, immédiatement après les faits.

" Les employés devraient avoir l'assurance que déclarer des incidents à la direction ou en débattre entre eux ou avec leurs représentants n'entraînera pour eux aucune conséquence négative.

- 14.c.4 L'information concernant les incidents devrait aussi être transmise aux associations professionnelles pertinentes.

14.c.5 Il faudrait s'efforcer de coordonner les déclarations faites par l'industrie à l'échelon national et international, afin de faciliter l'échange d'informations. 


\section{Chapitre 15}

\section{ENQUÊTES SUR LES INCIDENTS}

\section{a. PRINCIPES GÉNÉRAUX}

Ces principes généraux s'appliquent aux enquêtes menées tant par l'industrie que par les pouvoirs publics. Les objectifs des enquêtes peuvent varier selon les parties qui entreprennent celles-ci (par exemple, les pouvoirs publics peuvent procéder à une enquête à des fins d'application de la loi). Cependant, les enquêtes conduites par l'industrie et les pouvoirs publics ont en commun un certain nombre d'éléments, surtout au niveau des méthodes à utiliser.

\section{(Voir l'encadré à la suite du paragraphe 15.a.1 se rapportant aux enquêtes sur les incidents)}

15.a.1 La direction devrait enquêter sur tous les incidents liés à des substances dangereuses qui se produisent dans ses installations. Les pouvoirs publics devraient entreprendre une enquête sur les accidents ayant des effets nuisibles considérables.

4 Les objectifs des enquêtes initiées par l'industrie et ceux des enquêtes entreprises par les pouvoirs publics seront différents (voir les sections b et c ci-dessous). Cependant, nombre des principes à la base du déroulement de l'enquête seront semblables, comme on l'explique ici (section a).

" De manière générale, les enquêtes initiées par l'industrie seront indépendantes de celles entreprises par les pouvoirs publics, même si des enquêtes conjointes seraient souhaitables.

\section{ENQUÊTES SUR LES INCIDENTS}

L'enquête devrait être un processus de recherche de faits visant à tirer des leçons de l'expérience vécue, et non pas un exercice dont l'objectif serait de trouver un coupable ou un responsable. Ceux qui sont mêlés à l'incident ne devraient pas s'inquiéter à ce chapitre. Il devrait exister une totale collaboration entre le personnel d'exploitation des installations et les enquêteurs.

Dans le cadre des enquêtes, il faudrait mettre l'accent sur la détermination des causes sous-jacentes (parfois appelées les causes fondamentales) dans la chaîne des événements qui ont mené à l'accident, sur les leçons à tirer de l'incident et sur les moyens d'éviter des accidents semblables à l'avenir. L'enquête ne devrait pas se borner à établir les causes immédiates ou apparentes.

Il faudrait être conscient que les accidents constituent habituellement l'étape ultime d'une longue séquence d'événements où s'enchevêtrent, selon un motif complexe, défauts techniques, erreurs humaines et lacunes en matière d'organisation ou de gestion. 


\section{ENQUÊTES SUR LES INCIDENTS (suite)}

Il ne devrait pas suffire d'inscrire « erreur humaine » comme cause, le cas échéant. Les enquêteurs devraient plutôt déterminer exactement quels éléments ont un rôle à jouer dans l'erreur humaine. Il peut s'agir d'ennui, de stress, de surcharge de travail ou de manque de formation. Figurent aussi parmi les autres causes fondamentales possibles : une tolérance insuffisante du système aux erreurs; le défaut de fournir les procédures d'exploitation par écrit ou de les maintenir à jour; des procédures irréalistes ayant créé des circonstances fâcheuses ou exigé de l'opérateur qu'il agisse de façon illogique; une mauvaise conception de l'ergonomie, du système ou de la technologie; des moyens de fabrication fournissant trop ou trop peu de données à l'opérateur pour que l'on puisse s'attendre à une réponse appropriée de sa part; un manque de personnel; une pression inacceptable exercée sur l'opérateur ou le gestionnaire pour que la productivité prime sur la sécurité; une réorganisation ou un changement de personnel mal gérés.

Les erreurs humaines ne se limitent pas aux erreurs des opérateurs; elles peuvent se produire en différents points de la hiérarchie de l'entreprise, par exemple au niveau des responsables de la maintenance, des responsables de la gestion du changement ou des permis d'exploitation, ou encore au niveau de la supervision et de la direction. Parmi les exemples de causes d'erreurs humaines autres que celles attribuables aux opérateurs figurent les suivants : problèmes dans la transmission des connaissances, en particulier lorsque des employés spécialisés prennent leur retraite; complexité du système, y compris la conception et l'ingénierie des procédés; vieillissement des installations et réparations connexes, en l'absence d'inspections et de maintenance convenables; manque d'adaptation aux changements organisationnels et technologiques, dont l'automatisation.

La recherche des causes fondamentales devrait être systématique, exhaustive et équitable, et comprendre quatre phases principales :

- La première phase se situe avant qu'il soit possible d'accéder aux lieux de l'accident. Un certain nombre de mesures peuvent alors être prises pour faire avancer l'enquête; il s'agira notamment de mettre sur pied un groupe d'enquête, d'interroger les témoins oculaires, d'organiser un circuit d'information et de suivi, de dresser la liste des facteurs qui pourraient avoir eu une incidence sur les événements, d'établir une liste préliminaire de scénarios envisageables, de coordonner le travail avec l'équipe d'intervention d'urgence afin de préserver les éléments de preuve, d'entamer l'enquête à l'extérieur de la zone dont l'accès est restreint, de se préparer à l'arrivée d'un volume important de données et de prendre des photographies aériennes.

- La deuxième phase est la visite initiale des lieux; à ce moment, il est primordial de rassembler de l'information sur l'état du site, de revoir le plan d'enquête, et de relever les éléments de preuve qui risquent de s'altérer avec le temps.

- La troisième phase est celle de l'enquête; les efforts se concentrent alors sur le relevé des éléments de preuve, la reconstitution des événements, l'analyse, l'expérimentation et la simulation des scénarios, et l'infirmation ou la confirmation systématiques des hypothèses de scénarios.

- La quatrième phase comprend la rédaction du rapport d'enquête et des recommandations; ces documents devraient être achevés rapidement, de manière à éviter les délais dans l'application des mesures correctives.

Dans la planification et la conduite des enquêtes sur les causes fondamentales des incidents, il faudrait s'employer à tenir compte des éventuelles contraintes ou des obstacles possibles à l'efficacité des procédures d'investigation, comme :

- la disparition ou l'altération des éléments de preuve à cause des possibles troubles de mémoire des témoins oculaires avec le temps, et à cause du fait que l'enquête se déroule dans des conditions de grand stress, et peut se poursuivre pendant quelques mois;

- le nombre limité de scénarios examinés, ce qui introduit un biais dans la collecte des éléments de preuves retenus pour harmoniser les scénarios choisis;

- la législation visant à promouvoir l'accès du public à l'information et les lois conçues pour protéger les renseignements commerciaux confidentiels, qui peuvent constituer des entraves à l'obtention et au partage des éléments de preuve pertinents; 


\section{ENQUÊTES SUR LES INCIDENTS (suite)}

- le caractère limité des ressources humaines et financières, en regard de la complexité de l'enquête;

- le manque de confiance entre les parties concernées;

- les questions de responsabilité;

- les mesures à prendre pour assurer la sécurité du site.

L'analyse sera facilitée par l'emploi d'une base de données informatique où sont emmagasinés les éléments clés de chaque incident. On pourra ainsi faire ressortir des tendances particulières et utiliser les données rétrospectives de façon anticipative en vue de prévenir les accidents; on pourra, par exemple, orienter la formation en matière de sécurité vers la prévention du type d'incidents qui se sont produits.

\section{Éléments clés des enquêtes sur les causes fondamentales}

\section{(Voir l'encadré ci-dessus)}

15.a.2 Il faudrait établir des protocoles d'enquête sur les causes fondamentales. Ces protocoles devraient notamment définir les rôles et les responsabilités des personnes qui participent à l'enquête. Ils devraient également préciser les étapes du processus d'enquête.

n Les objectifs des enquêtes sur les causes fondamentales devraient être les suivants :

- découvrir ce qui s'est passé;

- déterminer pourquoi l'incident s'est produit (c'est-à-dire trouver les causes sous-jacentes et les causes connexes, et reconstituer la chaîne des événements);

- élaborer des plans d'application de mesures correctrices par la direction en vue de prévenir des incidents analogues ou identiques;

- $\quad$ mettre ces plans en œuvre.

W À cet égard, une fois la première étape accomplie (découvrir ce qui s'est passé), il est essentiel de ne pas cesser de demander "pourquoi? » tant que les causes sousjacentes ou fondamentales ne sont pas déterminées.

W Les protocoles devraient également préciser quand il convient de mettre un terme à l'enquête, pour garantir que celle-ci n'est pas conclue prématurément. Par exemple, de manière générale, le défaut de suivre les procédures ne devrait pas être considéré comme une cause fondamentale. Il faut comprendre à quoi l'incident est attribuable, à la base. De plus, l'incident en lui-même ne peut être tenu pour une cause fondamentale (par exemple, le fait qu'une explosion se soit produite ne peut être considéré comme la cause fondamentale du rejet de substances dangereuses).

15.a.3 Il faudrait mettre sur pied un groupe chargé d'enquêter sur l'accident.

n Le groupe devrait être pluridisciplinaire et compter des membres qui connaissent l'installation faisant l'objet de l'enquête. Ces derniers peuvent être des employés participant à l'exploitation ou à la maintenance de l'installation, ou leurs représentants. 
" Tous les membres du groupe d'enquête devraient avoir les connaissances, les compétences et l'expérience nécessaires pour mener des enquêtes et assumer les rôles et les responsabilités qui leur ont été confiés.

15.a.4 Il faudrait tenir compte, dans le cadre des enquêtes, des divers types d'information et d'éléments de preuve ${ }^{5}$ possibles, dont les témoignages, la documentation (sur place ou hors site, notamment les registres de maintenance, de laboratoire et d'échantillonnage ainsi que les relevés météorologiques) et les preuves matérielles (constatées sur les lieux de l'accident et sur l'équipement connexe).

" Dans la mesure du possible, les éléments de preuve devraient être protégés de manière à faciliter le processus d'enquête. Il est impératif de relever et de préserver tous les éléments de preuve pouvant s'altérer avec le temps, et de faire le nécessaire pour éviter que la chaîne de possession des éléments de preuve soit rompue. Il faudrait désigner sans équivoque les responsables de la garde des éléments de preuve et de leur divulgation.

" Par conséquent, les équipes d'intervention d'urgence devraient posséder la formation requise pour savoir comment préserver au maximum l'intégrité des éléments de preuve.

" Des mesures devraient être prises pour protéger efficacement les renseignements commerciaux confidentiels qui risquent d'être divulgués au cours de l'enquête, et tous les membres du groupe d'enquête devraient connaître ces mesures de protection et la manière dont elles devraient être appliquées.

- 15.a.5 Des rapports d'enquête devraient être rédigés; ils devraient notamment présenter la chronologie des événements ayant conduit à l'accident ou au quasi-accident, les causes sous-jacentes (ou fondamentales) ainsi que les causes connexes, de même que des recommandations quant aux mesures de suivi à appliquer. Il faudrait aussi expliquer dans ce rapport quelles théories relatives aux causes de l'accident sont erronées, et en quoi.

- 15.a.6 Les recommandations faites au terme d'une enquête devraient être spécifiques, de telle façon qu'elles permettent de rectifier les techniques ou les méthodes de gestion. En général, une enquête amènera toute une série de recommandations quant aux mesures à prendre (c'est-à-dire qu'habituellement, une intervention unique ne suffira pas). Sachant cela, l'objectif devrait être de chercher la solution optimale compte tenu des circonstances, en gardant à l'esprit qu'il se peut que la solution parfaite n'existe pas.

15.a.7 Un suivi adéquat devrait être donné à l'enquête afin de vérifier que les mesures correctrices ont été appliquées conformément aux recommandations, et qu'elles ont permis de remédier aux problèmes relevés.

15.a.8 Il faudrait considérer le recours à une tierce partie, par exemple des consultants, pour évaluer les résultats de l'enquête sur l'accident et vérifier la qualité des conclusions de l'enquête ainsi que des recommandations formulées dans le rapport.

15.a.9 Lorsque plus d'une instance a l'autorité de procéder à une enquête sur un accident, il faudrait s'efforcer de coordonner les enquêtes de manière à éviter les chevauchements, à accroître l'efficacité et à assurer l'accès à tous les éléments de preuve pertinents. 
15.a.10 Au terme d'une enquête, le processus d'investigation devrait être réexaminé pour vérifier son efficacité, pour voir si un suivi approprié a été effectué compte tenu des résultats de l'enquête, et pour tirer des leçons en vue d'enquêtes futures. Dans cette optique, il peut être très utile $\mathrm{d}^{\prime}$ associer des parties « externes » à ce processus de réexamen (notamment des consultants ou des spécialistes appartenant à des associations industrielles).

\section{Partage des résultats de l'enquête}

\section{(Voir les paragraphes $15 . b .3$ et 15.c.3)}

15.a.11 Il faudrait s'employer à promouvoir le partage des leçons tirées des enquêtes sur les incidents et à faciliter la diffusion des résultats aussi rapidement que possible (par exemple grâce à des technologies comme Internet).

" Il faudrait réaliser que la simple diffusion des «leçons apprises » peut être insuffisante, et s'efforcer de comprendre de quelle façon communiquer l'information pour que les mesures appropriées soient appliquées. Dans cette perspective, les «leçons apprises" peuvent représenter une composante extrêmement importante des programmes de formation et de sensibilisation.

"Il faudrait s'employer à détecter les obstacles au partage de l'information concernant les enquêtes et les leçons apprises (dont les soucis d'ordre juridique et les considérations liées à la protection des renseignements commerciaux confidentiels) et à les aplanir.

- 15.a.12 Les informations pertinentes des rapports d'enquête (comme les recommandations et les leçons apprises) devraient être communiquées aux diverses parties prenantes potentiellement concernées par ces résultats (par exemple, les utilisateurs des technologies visées par le rapport), mais dans le strict respect de la confidentialité de certains renseignements commerciaux. La diffusion des leçons apprises contribuera à faire en sorte que tous les employés concernés, dans toutes les installations visées, en tiennent compte.

" La direction devrait partager les aspects pertinents des rapports d'enquête aussi largement que possible avec les pouvoirs publics et avec les autres parties concernées à des fins d'amélioration de la sécurité et d'information du public. Chacune des parties concernées a tout intérêt à rendre publics, dans la mesure du possible, les éléments pertinents de ces rapports. De tels documents peuvent également être utilisés dans le cadre des activités de sensibilisation et de formation.

" Les entreprises devraient chercher à diffuser les éléments clés des leçons apprises, par l'intermédiaire des bases de données ou des centres d'échange nationaux ou internationaux à leur disposition.

" Il faudrait élaborer des mécanismes pour faire en sorte que les entreprises qui n'ont pas accès aux circuits réguliers d'information sur la sécurité (par exemple, les associations industrielles) reçoivent les renseignements pertinents concernant les expériences vécues dans d'autres entreprises.

" Les pouvoirs publics devraient faciliter la circulation des rapports au sein de l'industrie. À cette fin, ils devraient envisager la création de bases de données sur les 
incidents (accidents et quasi-accidents) de telle façon que les entreprises puissent avoir aisément accès à cette information (par exemple, grâce à Internet).

15.a.13 Il faudrait s'employer à améliorer le partage de l'expérience relative aux méthodes et démarches utilisées dans le cadre d'enquêtes sur des incidents.

15.a.14 Il faudrait chercher à mettre en place un cadre de travail élémentaire et une terminologie commune pour la préparation des rapports d'enquête afin de faciliter le partage de l'information relative aux enquêtes.

\section{b. INDUSTRIE}

15.b.1 La direction des installations dangereuses devrait s'assurer que tous les incidents mettant en cause des substances dangereuses font immédiatement l'objet d'une enquête et d'une analyse approfondie.

4 La direction des installations dangereuses devrait adopter des normes internes indiquant clairement la nature des enquêtes qui devraient être effectuées, les personnes qui devraient y participer, et les critères qui devraient être utilisés pour déterminer l'étendue de l'enquête selon le type d'incident.

- Les incidents (tant les accidents que les quasi-accidents) pouvant apporter les plus grandes améliorations au chapitre de la sécurité dans le cas des produits chimiques devraient faire l'objet d'enquêtes particulièrement minutieuses. Dans les cas où la probabilité de tirer des leçons de l'incident est moins grande, l'enquête devrait être moins poussée.

" Il est important d'enquêter sur les quasi-accidents puisqu'ils sont souvent annonciateurs d'accidents potentiels, et peuvent mettre en lumière les mesures qu'il convient de prendre pour éviter ces derniers. De plus, les enquêtes sur les quasiaccidents engagent les employés et contribuent à répartir les responsabilités en matière de sécurité. Pour améliorer la compréhension des quasi-accidents, il faut d'abord que ceux-ci soient décelés (par exemple, par un employé), puis que la situation soit rapportée aux parties concernées. La direction devrait encourager la détection et la déclaration des quasi-accidents en créant un climat de confiance dans lequel les employés ne craindront pas d'être blâmés, et en exprimant nettement le message que la déclaration des quasi-accidents est essentielle. Enfin, la procédure de déclaration des quasi-accidents devrait être relativement simple.

" Les personnes qui participent aux enquêtes (menées à l'interne ou par une tierce partie) devraient avoir l'autorité et les ressources requises pour prendre toute mesure corrective dictée par les résultats de l'enquête.

" Afin d'améliorer la sécurité des installations dangereuses, il faudrait constituer un dossier d'enquête et publier le rapport d'enquête pour informer toutes les parties concernées des leçons apprises.

15.b.2 La direction devrait être déterminée à conduire une enquête sur les causes fondamentales en vue d'en tirer des leçons permettant d'améliorer la sécurité. 
" Des ressources suffisantes devraient être allouées pour mener à bien ce travail et appliquer les mesures correctives recommandées dans les rapports d'enquête.

" Les enquêtes sur les causes fondamentales devraient se poursuivre au moins jusqu'à l'établissement des causes dont la suppression empêchera que se reproduisent des événements pouvant mener au même type ou à un type analogue d'accident ou de quasi-accident.

- 15.b.3 La direction devrait diffuser les résultats des enquêtes (ce qui comprend les recommandations et les leçons apprises) sur les accidents et les quasi-accidents au sein de l'entreprise et les communiquer aux autres entreprises de même qu'aux autres parties concernées, afin d'aider à éviter que surviennent les mêmes problèmes ou des problèmes similaires à l'avenir.

" Il est important que les leçons apprises soient transmises à l'ensemble des effectifs de l'entreprise (à la direction aussi bien qu'aux travailleurs et à leurs représentants) et, en particulier, aux autres installations de l'entreprise qui risquent de faire face à des situations semblables.

" Les leçons apprises devraient être communiquées aux personnes qui ont le pouvoir de procéder aux changements recommandés dans le rapport d'enquête. Il faudrait que chacun dans l'entreprise s'engage à prendre les mesures qui s'imposent à la lumière des recommandations et des leçons apprises.

" Les rapports d'enquête et les leçons tirées des incidents devraient être conservés adéquatement, de façon qu'il soit possible de les consulter facilement et en tout temps. Cela devrait contribuer à préserver la mémoire de l'entreprise dans le temps, même en cas de changement substantiel de personnel.

" Tous les nouveaux employés devraient recevoir une formation comprenant une présentation des éléments pertinents des accidents et des quasi-accidents qui se sont produits dans le cadre des activités de leur entreprise et un exposé des leçons tirées de ces incidents.

" Les résultats des enquêtes devraient être mis à la disposition des autres entreprises à qui cette information pourrait être profitable pour améliorer la sécurité de leurs installations (par exemple, les entreprises possédant des installations analogues à celles où l'incident s'est produit).

" La diffusion de l'information contenue dans les rapports d'enquête devrait se faire selon des procédures établies, qui prévoient notamment des moyens de retracer le cheminement de l'information et d'en faire le suivi.

\section{(Voir les paragraphes 15.a.11 - 14)}

15.b.4 La direction devrait chercher, de manière anticipative, à connaître les expériences pertinentes vécues par d'autres entreprises en matière d'enquête et à en tirer profit. Les renseignements relatifs à ces expériences peuvent se trouver, par exemple, dans les rapports d'accident affichés sur les sites des entreprises, dans les bases de données nationales et internationales ou dans d'autres sources d'information accessibles aux entreprises. 


\section{c. POUVOIRS PUBLICS}

15.c.1 Les pouvoirs publics devraient mener une enquête indépendante sur tous les accidents ayant des effets nuisibles importants sur la santé, l'environnement ou les biens, de même que sur les accidents pouvant fournir des indications substantielles au chapitre de la réduction des risques. Cela devrait faire partie du rôle qu'ont les pouvoirs publics, à titre d'autorité réglementaire, de déterminer les causes sous-jacentes (ou fondamentales) et les causes connexes des accidents.

" Les pouvoirs publics devraient également envisager l'ouverture d'une enquête lorsqu'ils soupçonnent qu'une loi ou un règlement n'ont pas été respectés.

- Il faudrait constituer un dossier d'enquête et publier les informations pertinentes des rapports d'enquête afin d'informer les parties concernées des leçons apprises, et ainsi améliorer la sécurité des installations dangereuses.

" Les enquêtes menées par les pouvoirs publics devraient être impartiales et dignes de foi, afin que le public soit convaincu de leur bien-fondé.

* Le cas échéant, l'enquête sur l'accident devrait être conduite par un groupe de spécialistes formé de personnes autres que celles responsables de l'inspection à l'installation et de l'application du cadre réglementaire (par exemple, une commission spéciale).

" Toutes les parties intéressées devraient avoir l'occasion de participer à l'enquête.

- Les pouvoirs publics devraient avoir la responsabilité de veiller à ce que soient prises les mesures qui s'imposent à la lumière des recommandations formulées dans les rapports d'enquête.

15.c.2 Les pouvoirs publics devraient établir des critères d'après lesquels ils fixeront les priorités en matière d'enquête (c'est-à-dire quels accidents devraient faire l'objet d'une enquête et dans quelle mesure), compte tenu des limitations en ressources.

- Les critères devraient être choisis de manière à maximiser les bénéfices (par exemple en termes de leçons à tirer), à utiliser les ressources de façon optimale et à permettre de prendre des mesures et d'obtenir des résultats rapidement.

- Dans cette perspective, les pouvoirs publics devraient prendre en considération des facteurs comme l'historique en matière d'accidents analogues, la portée des dommages à la santé et à l'environnement, le nombre d'établissements où sont utilisés les procédés en cause dans l'accident, et la probabilité que l'acquisition de nouvelles informations aboutisse à une amélioration de la sécurité. De plus, il faudrait tenir compte d'intérêts stratégiques, comme le niveau de préoccupation du public à l'égard de la situation.

15.c.3 Les rapports d'enquête préparés par les pouvoirs publics devraient, une fois apportées les modifications nécessaires à la protection des renseignements confidentiels, être publiés en vue d'une diffusion aussi large que possible. 
" Les rapports devraient contenir suffisamment de renseignements généraux pour que les résultats de l'enquête soient utiles dans d'autres situations.

" Les rapports devraient présenter les conclusions dégagées de l'analyse des données sur l'accident.

" Les pouvoirs publics devraient communiquer ces rapports aux associations industrielles de leur pays susceptibles de tirer profit des leçons apprises de l'enquête.

" Il faudrait élaborer un mécanisme qui facilite la circulation des rapports d'enquête à l'échelon international et, plus particulièrement, qui améliore le partage de l'information relative aux causes des accidents.

" Les pouvoirs publics sont les mieux placés pour établir des corrélations entre les données contenues dans les rapports d'enquête, favoriser l'échange des renseignements et produire des analyses crédibles. Ces éléments sont importants à la fois pour les pouvoirs publics et pour la direction des installations dangereuses aux fins de l'amélioration des processus décisionnels (par exemple, pour l'élaboration et l'application de la réglementation, le contrôle, la préparation de plans d'urgence et la mise au point de techniques de gestion et d'évaluation des risques).

\section{(Voir aussi les paragraphes 15.a.11 - 14)}

15.c.4 Les pouvoirs publics devraient disposer des ressources nécessaires pour assumer leurs responsabilités en matière d'enquêtes sur les accidents et de diffusion des renseignements connexes à ces activités.

15.c.5 Lorsque plus d'une instance (nationale, régionale et [ou] locale) prend part à l'enquête, il faudrait s'efforcer de coordonner les activités des organismes engagés dans l'investigation.

" Une telle coordination optimisera la capacité des témoins à fournir des renseignements utiles, limitera les perturbations dans l'exploitation, renforcera l'impartialité, garantira la cohérence des éléments de preuve tirés des échantillons et améliorera l'efficacité de l'enquête.

" Il est nécessaire de discuter de la coordination et de la collaboration et de s'entendre sur ces questions avant le début de l'enquête. Les objectifs des divers organismes, qui diffèrent d'une instance à l'autre, devraient être pris en compte dans l'élaboration des mécanismes de coordination.

\section{d. AUTRES PARTIES PRENANTES}

15.d.1 Des représentants des collectivités devraient être associés aux séances de débreffage et aux enquêtes sur les accidents, de même qu'à l'examen des rapports d'enquête, s'il y a lieu, pour aider à réduire la probabilité que des accidents analogues se reproduisent, et à améliorer les mesures de préparation et d'intervention. D'autres intervenants peuvent également contribuer à accroître la diffusion des leçons tirées des enquêtes sur les accidents. À titre d'exemple, il faudrait que les associations techniques et professionnelles, les milieux universitaires et d'autres organisations non 
gouvernementales prennent part au processus de diffusion des renseignements pertinents.

15.d.2 Lorsque des compagnies d'assurance entreprennent des enquêtes sur les accidents, les résultats de leurs investigations devraient être transmis à l'entreprise concernée et aux autres entreprises, dans la mesure du possible. 


\section{NOTES}

1. Voir la Recommandation du Conseil de l'OCDE C(89)88(Final).

2. Comme on l'a déjà souligné, plusieurs projets à l'échelle nationale et internationale visant à améliorer la connaissance des effets sur la santé de l'exposition aiguë à certains produits chimiques sont en cours. Parmi ces projets, on compte le projet de détermination des AEGL, lancé par les États-Unis et regroupant maintenant plusieurs pays européens, et le projet ACUTEX de la CE. Ces initiatives ont pour objectif d'élaborer des méthodes nouvelles permettant d'établir des seuils d'exposition aiguë applicables dans le cadre de la planification pour les situations d'urgence et l'aménagement du territoire.

3. L'Organisation mondiale de la Santé poursuit actuellement des travaux relatifs à un partage $\mathrm{d}^{\prime}$ information de ce type.

4. Il existe des mécanismes internationaux de partage des statistiques et des études de cas sur les accidents. En particulier, il y a la base de données MARS (Major Accident Reporting System), gérée par le Major Accident Hazards Bureau (MAHB) de la Commission européenne, qui compile les rapports d'accidents provenant des pays de l'Union européenne. De plus, MARS est utilisé pour gérer le mécanisme de déclaration des accidents mis au point par l'OCDE de même que celui élaboré par la CEE-ONU.

5. On peut donner à "élément de preuve » la définition suivante : «tout élément requis pour évaluer les scénarios et appuyer l'analyse ». 


\section{Partie E}

\section{QUESTIONS SPÉCIALES}

Cette partie complète les parties $A$ à $D$ des Principes directeurs en fournissant des lignes d'orientation supplémentaires, plus spécifiques, qui se rapportent:

- aux questions transfrontalières ou internationales, y compris la coopération transfrontalière, l'aide internationale, le transfert de technologie et l'investissement international (chapitre 16);

- au transport mettant en jeu des installations fixes associées au transport de substances dangereuses, comme les interfaces des transports (dont les zones portuaires) et les pipelines (chapitre 17).

Chacun de ces chapitres est décrit de manière plus détaillée ci-après. 


\section{Chapitre 16}

\section{QUESTIONS TRANSFRONTALIÈRES/INTERNATIONALES}

Le présent chapitre traite d'un certain nombre de questions concernant les relations entre différents pays dont la coopération internationale relative à des installations dangereuses situées près des frontières, de même que l'aide bilatérale et multilatérale touchant la prévention, la préparation et l'intervention en matière d'accidents chimiques. De plus, il aborde la question du transfert de technologie et de l'investissement international dans la technologie associée aux installations dangereuses.

Le présent chapitre traite aussi du rôle des organisations intergouvernementales au chapitre de la prévention, de la préparation et de l'intervention en matière d'accidents chimiques (voir l'encadré suivant le paragraphe 16.b.15).

Le présent chapitre est fondé sur le postulat voulant que toutes les installations dangereuses, sans égard à leur emplacement, devraient atteindre le même niveau de sécurité, et que toutes les dispositions des Principes directeurs s'appliquent aux installations dangereuses du monde entier. Cependant, il est entendu que la mise en cuvre intégrale des Principes directeurs peut être impossible dans certaines collectivités à cause du caractère limité des ressources humaines et financières, du manque d'information ou des lacunes du cadre juridique.

Le présent chapitre considère également le rôle particulier des pays développés, compte tenu de leur expérience au niveau de la prévention, de la préparation et de l'intervention en matière d'accidents chimiques. Par conséquent, il faudrait trouver des moyens pour faciliter la communication de l'information et du savoirfaire aux pays qui ne possèdent pas le même degré de connaissances ni la même expérience dans ce domaine.

À cet égard, l'aide aux pays en développement et aux pays à économie en transition devrait être compatible avec un développement respectueux de l'environnement; il faudrait donc s'efforcer d'éviter autant que possible que les projets d'aide ou le transfert de technologie entraînent des risques déraisonnables d'accident chimique.

Le présent chapitre s'inspire d'ententes internationales (comme les Conventions de l'OIT et de la CEE-ONU), de même que de documents d'orientation rédigés par le PNUE, le BCAH, l'OMS, l'OCDE, la CE et d'autres instances.

\section{a. COOPÉRATION TRANSFRONTALIÈRE}

16.a.1 Les pays voisins devraient échanger l'information et se consulter pour prévenir les accidents susceptibles de provoquer des dommages transfrontaliers et pour réduire les effets nuisibles d'un éventuel accident.

" À cette fin, un pays, où une installation dangereuse est établie ou projetée ("pays hôte »), devrait transmettre à tous les pays susceptibles d'être touchés les renseignements pertinents au sujet de cette installation; en retour, les pays potentiellement touchés devraient communiquer au pays hôte toute information pertinente à propos du secteur de leur territoire qui pourrait être touché par les dommages transfrontaliers en cas d'accident.

" Les renseignements que le pays hôte devrait fournir, dans la mesure où ils sont disponibles et en conformité avec ses lois, comprennent : 
- l'emplacement et la description générale de l'installation dangereuse qui pourrait causer des dommages transfrontaliers;

- le nom commun ou, si cela convient mieux, le nom générique ou la classe générale de danger des substances dangereuses qui pourraient être à la source d'effets transfrontaliers;

- les exigences légales, réglementaires et administratives auxquelles est soumise l'exploitation;

- les renseignements généraux quant à la nature, à la portée et à la probabilité des effets hors site d'un accident sur la santé, l'environnement ou les biens;

- l'information sur les aspects pertinents du plan d'urgence externe.

"Les pouvoirs publics des pays susceptibles d'être touchés devraient transmettre les renseignements suivants aux pouvoirs publics du pays hôte :

- la répartition de la population, notamment des groupes vulnérables;

- l'emplacement et la description générale des biens et des activités qui pourraient subir des effets nuisibles;

- l'emplacement des ressources naturelles, des zones protégées, des milieux sensibles et des monuments historiques qui pourraient être endommagés.

- 16.a.2 En ce qui concerne l'aménagement du territoire dans la perspective de projets de construction d'installations dangereuses pouvant causer des dommages transfrontaliers en cas d'accident, une politique d'échange de l'information et de consultation avec les pouvoirs publics compétents des pays voisins devrait être appliquée conformément à la Convention sur les effets transfrontaliers des accidents industriels de la CEE-ONU, à la Convention sur la protection et l'utilisation des cours d'eau transfrontaliers et des lacs internationaux de la CEE-ONU, et enfin à la Décision du Conseil de l'OCDE C(88)84(Final).

16.a.3 Les pays voisins devraient se consulter afin de coordonner les plans d'urgence externes relatifs aux installations dangereuses pouvant causer des dommages transfrontaliers.

" Ils devraient s'informer mutuellement sur les voies de communication à utiliser, des grandes lignes de leurs plans d'urgence respectifs et des moyens d'intervention d'urgence à leur disposition en cas d'accident causant des dommages transfrontaliers.

" Ils devraient s'informer mutuellement sur les instructions données à leurs populations respectives sur ce qu'il convient de faire en cas d'accident.

- 16.a.4 Les pays voisins devraient établir des procédures qui assurent une transmission rapide et efficace de l'information relative à un accident (ou à une menace imminente d'accident) pouvant entraîner des effets transfrontaliers, et ils devraient mettre en place des systèmes destinés à la communication des renseignements pertinents en cas d'accident.

" Les pays concernés devraient s'informer mutuellement sur l'identité des autorités responsables de la transmission et de la réception des renseignements, et sur les autorités responsables de l'application des plans d'urgence externes.

" Les pays concernés devraient collaborer pour faire en sorte que les populations pouvant être touchées par un accident reçoivent toutes les mêmes informations, qu'elles soient dans le pays hôte ou dans un pays voisin. 
" Les pouvoirs publics qui reçoivent les renseignements sur les installations dangereuses devraient respecter la confidentialité des données transmises. Ils ne devraient pas diffuser des renseignements qui ne sont pas du domaine public dans le pays d'où ils proviennent.

16.a.5 En cas d'accident lié à des substances dangereuses (ou en cas de menace imminente d'un tel accident) pouvant provoquer des effets transfrontaliers, les pouvoirs publics du pays hôte devraient veiller à ce que les autorités compétentes des pays pouvant être touchés reçoivent sans délai les renseignements appropriés. De plus, le pays hôte devrait s'employer à coordonner les mesures d'intervention avec celles mises en œuvre dans les pays voisins. Les renseignements que le pays hôte devrait transmettre aux pays risquant d'être touchés devraient décrire, par exemple :

- le lieu et les circonstances (brève description) de l'accident;

- les effets immédiats de l'accident;

- les mesures d'urgence prévues et l'intervention effectuée;

- la nature et la quantité de substances dangereuses qui pourraient toucher les pays potentiellement vulnérables, ainsi que la forme sous laquelle elles se présentent;

- les données disponibles pour évaluer les répercussions probables de l'accident.

16.a.6 Les représentants des pays ou des collectivités potentiellement vulnérables devraient avoir l'occasion de participer aux procédures de délivrance des permis aux installations dangereuses pouvant provoquer des effets transfrontaliers chez eux, ainsi qu'aux procédures pour le choix du site.

- 16.a.7 Dans la mesure du possible, les pouvoirs publics devraient tenter de prêter main-forte aux autres pays qui sollicitent leur aide au chapitre de la préparation et de l'intervention en matière d'accidents mettant en cause des substances dangereuses.

16.a.8 Les pouvoirs publics devraient élaborer des procédures pour faciliter le transit des ressources humaines et matérielles sur leur territoire à des fins d'entraide en cas d'accident lié à des substances dangereuses.

16.a.9 Les pouvoirs publics devraient faciliter l'échange des technologies liées à la prévention, à la préparation et à l'intervention en matière d'accidents chimiques.

\section{b. AIDE TECHNIQUE ET FINANCIÈRE, BILATÉRALE OU MULTILATÉRALE}

\section{Principes généraux}

16.b.1 Il faudrait renforcer la coopération technique entre les pays développés et les «pays bénéficiaires " (c'est-à-dire les pays en développement et les pays à économie en transition [«PET»] ${ }^{1}$ afin d'accroître la capacité institutionnelle du gouvernement des pays bénéficiaires à assumer ses rôles et responsabilités en matière de sécurité des installations dangereuses. Dans cette perspective, une aide pourrait être apportée, par exemple, dans les domaines suivants : mise en place de programmes de prévention des accidents, planification de mesures d'urgence, intervention en cas d'accident, et support pour l'assistance en cas d'accident. 
- 16.b.2 Tous les pays donateurs et les pays bénéficiaires devraient chercher à améliorer l'efficacité des programmes d'aide.

4 Pour être plus efficaces, les programmes d'aide devraient:

- répondre à des besoins spécifiques, bien définis (c'est-à-dire en fonction de la demande) et être axés sur les résultats;

- recourir aux spécialistes locaux et aux langues locales;

- adopter une perspective à long terme;

- prévoir la participation de toutes les parties concernées, soit les pouvoirs publics, l'industrie (y compris les travailleurs) et les organismes communautaires.

" Tous les programmes devraient être bien planifiés et se voir allouer des ressources (humaines et financières) suffisantes pour permettre un suivi approprié.

" Il faudrait, si possible, utiliser les documents d'orientation et de formation existants, et toute la documentation pertinente devrait être diffusée aussi largement que possible.

" Dès le départ, il faudrait s'employer à définir les objectifs des programmes d'aide, leur public cible et un éventail approprié de participants.

" Toutes les parties concernées devraient être mobilisées dès le stade de la planification, de manière à déterminer l'engagement de chacune et à adapter la participation au type d'activité.

" Les participants aux programmes d'aide devraient être étroitement associés à l'élaboration, à la mise en œuvre et au suivi des mesures d'aide.

16.b.3 Les pays donateurs tout comme les pays et les organisations bénéficiaires devraient chercher à améliorer le partage et la coordination de l'information ainsi que la transparence et la responsabilisation afin d'éviter la duplication des tâches et d'accroître l'efficacité de tous les programmes.

" De cette manière, les organisations ou les pays pourraient tirer le meilleur parti des ressources dont ils disposent et exploiter leurs atouts.

" Cela permettrait aux pays donateurs de mieux circonscrire les programmes d'aide aux domaines prioritaires ( $c^{\prime}$ est-à-dire de faire en sorte $q^{\prime}$ 'ils soient davantage fonction de la demande) et d'associer toutes les parties concernées aux activités d'assistance.

" Cela permettrait également aux pays ou aux organisations bénéficiaires d'améliorer l'accès aux programmes et aux documents disponibles ainsi que leur utilisation, tout en réduisant la confusion qui pourrait naître d'éventuels messages contradictoires de la part de différentes organisations.

" Ainsi, le temps et les ressources des participants seraient mieux employés et les projets ainsi que les résultats seraient plus efficaces puisqu'on exploiterait les connaissances spécialisées d'une variété plus grande de secteurs que si les activités n'étaient pas concertées; en outre, les désaccords sur ce qui constitue les «meilleures » lignes d'orientation seraient ainsi éliminés. 
" Afin d'améliorer la transparence et la coordination, il faudrait mettre sur pied des centres de liaison et des mécanismes de coordination entre les pays donateurs, les pays bénéficiaires et les organisations internationales, de même qu'au sein de chacune de ces instances. Les mécanismes pourraient comprendre notamment: le partage, sur une base régulière, du calendrier des activités; la mise à jour et la diffusion périodiques des inventaires des technologies disponibles (ou prévues) pour la prévention, la préparation et l'intervention en matière d'accidents; l'examen scrupuleux des mandats, des activités et des objectifs relatifs aux projets, y compris les forces et les limites de ces derniers.

" La supervision des projets devrait être confiée aux organismes les mieux placés pour assumer ce rôle et, s'il y a lieu, il faudrait entreprendre des activités conjointes ou en coopération.

" Dans la mesure du possible, il faudrait avoir recours aux mécanismes de coordination existants, par exemple le Forum intergouvernemental sur la sécurité chimique (FISC) et le Programme interorganisations pour la gestion rationnelle des produits chimiques (IOMC).

\section{Rôle des organismes d'aide (nationaux et multinationaux) ${ }^{2}$}

- 16.b.4 Les organismes d'aide bilatéraux et multilatéraux et les entités connexes (globalement appelés " organismes d'aide») devraient aider les pays en développement et les PET à diminuer le plus possible les risques d'accident lié à des substances dangereuses par l'intermédiaire de leurs projets d'aide au développement.

" Il faudrait fournir de l'assistance technique et de la formation en vue de mettre en place des infrastructures institutionnelles, d'enrichir les capacités des ressources humaines et d'accroître la capacité institutionnelle dans les pays en développement et les PET. Cela aiderait les pouvoirs publics des pays bénéficiaires à remplir leurs rôles et responsabilités pour assurer la sécurité des installations dangereuses, y compris au chapitre de la prévention, de la préparation et de l'intervention en matière d'accidents.

" Les organismes d'aide devraient veiller à ce que les projets d'aide n'accroissent ni n'entretiennent le risque excessif d'accident lié à des substances dangereuses.

16.b.5 Les organismes d'aide devraient procéder à un examen critique des propositions d'aide pertinentes en vue de réduire autant que faire se peut la possibilité que des projets d'aide contribuent à créer, à accroître ou à entretenir un risque excessif d'accident lié à des substances dangereuses. Un projet convenable devrait plutôt viser l'objectif voulant que les installations dangereuses des pays en développement et des PET présentent un niveau de sécurité équivalent à celui des installations similaires situées dans les pays développés.

" Les organismes d'aide qui sont à l'origine de propositions d'aide devraient être sensibles aux problèmes de sécurité que posent ces propositions.

" L'équipe chargée, au sein d'un organisme d'aide, d'élaborer les propositions d'aide devrait, s'il y a lieu, s'adjoindre des spécialistes qui possèdent les antécédents, la 
formation et l'expérience voulus pour examiner les conséquences possibles en matière de sécurité de toute proposition relative à des installations dangereuses.

" Les organismes d'aide devraient recourir à des procédures officielles et explicites pour évaluer les risques potentiels d'accident lorsque sont prises des décisions concernant l'aide technique et financière relative à des installations dangereuses déterminées. Cette évaluation devrait entre autres prendre en compte les facteurs suivants : possibilités de défaillances techniques; capacités de gestion; compétences de la main-d'œuvre; caractère approprié de la technologie pour la collectivité locale; dispositions institutionnelles pour la surveillance, la préparation aux situations d'urgence et l'intervention en cas d'urgence. Les résultats de ces évaluations devraient être mis à la disposition des autorités des pays bénéficiaires et des groupes communautaires locaux.

" Lorsqu' on entreprend l'élaboration, l'évaluation et la mise en œuvre de projets d'aide concernant des installations dangereuses, il faudrait chercher à obtenir, dans les pays bénéficiaires, la participation des citoyens, notamment les dirigeants communautaires, afin de tirer profit de leur connaissance des atouts et des limites propres aux collectivités locales (infrastructure, compétences de la main-d'œuvre, facteurs culturels, etc.)

16.b.6 Dans la répartition des fonds alloués par des organismes d'aide relativement à des installations dangereuses, il faudrait veiller à ce qu'on dispose de ressources suffisantes pour les postes qui intéressent la sécurité, comme la sensibilisation et la formation. On devrait aussi songer à prévoir des dispositions et des fonds pour assurer dans de bonnes conditions le contrôle, l'évaluation, la maintenance et d'autres activités de suivi, de façon à répondre aux exigences essentielles en matière de sécurité.

16.b.7 Lorsqu'ils financent des projets industriels et autres programmes de développement mettant en œuvre des substances dangereuses, les organismes d'aide devraient veiller à ce que soient accessibles les services médicaux d'urgence et l'information médicale nécessaires pour le traitement des personnes blessées lors d'accidents chimiques. Il faudrait inciter les organismes d'aide à contribuer (par exemple par du financement) au développement, dans les pays bénéficiaires, des capacités d'intervention médicale indispensables en cas d'accident lié à des substances dangereuses.

16.b.8 Les organismes d'aide devraient fournir des informations, de l'enseignement technique, de la formation et de l'assistance en vue de promouvoir la sécurité des installations dangereuses à l'échelle locale.

" Cette assistance devrait, par exemple, avoir trait à des politiques concernant le choix des sites et l'aménagement du territoire qui évitent le peuplement des zones voisines des installations dangereuses, de même qu'à l'application des ententes et documents $\mathrm{d}^{\prime}$ orientation internationaux tels que ceux produits par le PNUE, l'OIT, l'OMS, le $\mathrm{BCAH}$, la CEE-ONU et l'OCDE.

4 Les organisations intergouvernementales, l'industrie, ainsi que les syndicats et leurs organisations internationales devraient prêter leur concours à la coopération technique. 
16.b.9 Les organismes d'aide, les associations industrielles et les entreprises des pays développés devraient aider les pays en développement et les PET à trouver les sources appropriées d'aide technique et financière à l'appui des activités relatives à la prévention, à la préparation et à l'intervention en matière d'accidents.

16.b.10 Les responsables de l'organisation des projets d'aide devraient régulièrement réexaminer d'un œil critique leurs programmes de travail pour vérifier qu'ils demeurent aussi efficaces que possible et qu'ils font une utilisation optimale des ressources disponibles.

"Dans le cadre du réexamen, il faudrait considérer, par exemple : l'efficacité globale des programmes; les outils existants de soutien aux programmes; s'il y a des avantages à adopter des démarches différentes; si les programmes continuent de répondre aux besoins premiers du public cible et de prendre en compte les conditions particulières propres à ce public; comment l'organisation des programmes et la coordination avec d'autres programmes peuvent être améliorées afin de réaliser de manière plus efficace les projets qui sont réclamés et qui peuvent être utilisés dans la pratique.

" Le réexamen régulier peut favoriser la responsabilisation et constituer la base pour déterminer s'il y a des leçons à tirer en vue d'améliorer les activités futures.

" Les organismes d'aide devraient être préparés à admettre que certains projets (y compris les propositions de nouveaux projets et les activités en cours) ne sont pas toujours nécessairement appropriés. Il se peut que le projet ne convienne pas à un site donné, qu'un autre donateur serait mieux placé pour fournir l'aide nécessaire, ou que le projet ne constitue pas une priorité pour le pays bénéficiaire.

16.b.11 Les réseaux sectoriels devraient être améliorés et mieux utilisés, de manière à faciliter la communication de l'information entre les spécialistes, à mettre sur pied des programmes coopératifs (par exemple, entre les instituts de recherche) et de procéder à des échanges d'inspecteurs et d'autres spécialistes.

\section{Rôle des institutions financières internationales}

- 16.b.12 Les institutions financières internationales (comme la Banque mondiale et les banques de développement régional) devraient élaborer et mettre en pratique des politiques et des procédures visant à réduire le plus possible les risques d'accident dans les installations dangereuses qu'elles aident à financer. Elles ne devraient, par exemple, participer à aucun projet qui présente des risques inacceptables d'accident lié à des substances dangereuses.

" À cet égard, une évaluation appropriée des risques d'accident, conforme aux présents Principes directeurs, devrait être réalisée avant que les institutions financières internationales ne subventionnent de nouvelles installations dangereuses ou l'agrandissement d'installations existantes.

" En outre, ces institutions devraient aider les pays en développement et les PET, s'il y a lieu, à entreprendre l'analyse des risques présents dans les installations existantes et à élaborer des programmes de sensibilisation et de formation aux mesures de prévention, de préparation et d'intervention applicables aux accidents. 
16.b.13 Les institutions financières internationales devraient informer le gouvernement des pays bénéficiaires chaque fois qu'un projet qu'elles envisagent de financer peut créer, accroître ou entretenir un risque d'accident mettant en cause des substances dangereuses, et devraient fournir tous les renseignements dont elles disposent à propos de ce risque.

16.b.14 Les institutions financières internationales devraient encourager la mise en œuvre de pratiques appropriées en matière de sécurité chez les entreprises qui bénéficient d'une aide financière relative à un transfert de technologie comportant certains risques d'accident liés aux substances dangereuses. Cela peut se faire en incitant les entreprises à se conformer aux présents Principes directeurs, et en tenant compte - au moment de déterminer le montant de l'aide financière - des ressources nécessaires à cette fin.

\section{Rôle des organisations intergouvernementales}

- 16.b.15 Les organisations intergouvernementales et leurs pays membres devraient évaluer de manière critique les besoins des pays bénéficiaires potentiels avant de créer de nouvelles entités ou de nouveaux programmes. Ils devraient tenir compte de la possible duplication des activités, et déterminer si un autre groupe serait mieux placé pour se charger des travaux envisagés.

\section{LE RÔLE DES ORGANISATIONS INTERGOUVERNEMENTALES}

Les organisations intergouvernementales ont un rôle important à jouer, soit d'exercer leur influence sur la mise en œuvre de pratiques de gérance des produits chimiques, comme celles qui sont formulées dans les présents Principes directeurs, d'encourager cette mise en œuvre, de promouvoir l'utilisation des outils et documents d'orientation à l'appui de ce processus, et de faciliter l'accès à ceux-ci. Les organisations intergouvernementales peuvent, en particulier, servir de relais entre les pays développés et les pays en développement ou à économie en transition, en vue de partager des leçons apprises et de veiller à ce que les pays puissent tirer profit des nombreuses ressources techniques et connaissances spécialisées dont on dispose en matière de gestion des produits chimiques. Les organisations intergouvernementales peuvent notamment :

- relayer l'information et l'aide entre donateurs et bénéficiaires;

- mobiliser et coordonner l'aide internationale aux pays qui font face à des situations d'urgence, surtout lorsque les capacités intérieures sont dépassées et (ou) que des évaluations neutres et indépendantes sont requises;

- combler, au besoin, les lacunes des ententes bilatérales et multilatérales (c'est-à-dire constituer un filet de sécurité international);

- appuyer et (ou) mettre en œuvre des projets visant le renforcement des capacités (à l'échelle locale et régionale);

- favoriser et soutenir l'élaboration de procédures et outils destinés à l'application des lignes d'orientation pertinentes;

- coordonner l'aide multilatérale pour répondre aux besoins et éviter les redondances;

- faciliter la coopération, la collaboration, l'assistance, le soutien financier, et l'accès aux connaissances techniques;

- accroître la sensibilisation à l'importance de recourir aux programmes et outils pertinents qui existent déjà pour faciliter les éléments énumérés ci-dessus;

- appuyer et parrainer l'application et l'adaptation de programmes et d'outils pour les pays en développement;

- entreprendre ou financer des examens de la performance en matière de sécurité à l'échelle mondiale;

- s'assurer d'une participation suffisante des parties prenantes internationales aux activités de prévention, de préparation et d'intervention; 


\section{LE RÔLE DES ORGANISATIONS INTERGOUVERNEMENTALES (suite)}

- faciliter le transfert de technologie à ceux qui en ont le plus besoin (entre les pays développés et les pays en développement);

- établir un lien pratique entre l'industrie et les organisations professionnelles pour promouvoir une plus grande utilisation par l'industrie des documents d'orientation ainsi que des systèmes relatifs à la santé, à la sécurité et à l'environnement de portée internationale, et enfin pour faciliter la normalisation, à l'échelle internationale, des outils et systèmes de déclaration et de gestion.

\section{INFORMATION ET PRÉPARATION AU NIVEAU LOCAL}

L'évaluation des circonstances entourant divers accidents industriels - comme celui de Bhopal (Inde), en 1984, les explosions de gaz de pétrole liquéfié à Mexico, en 1984, l'incendie d'un entrepôt de la société Sandoz, près de Bâle (Suisse), en 1986, l'explosion d'un dépôt de feux d'artifice à Enschede (Pays-Bas), en 2000, et l'explosion survenue à Toulouse (France), en 2001 - a mené à la conclusion que si les populations vivant près des installations à l'origine de ces accidents avaient été davantage sensibilisées et préparées, les répercussions néfastes de ceux-ci auraient été moindres.

APELL («Information et préparation au niveau local») est un outil créé par le PNUE pour éviter le plus possible les accidents technologiques et les situations d'urgence avec leurs conséquences néfastes, en accroissant la sensibilisation des collectivités locales et en améliorant la communication entre les divers intervenants. Il définit un processus bien structuré et détaillé pour l'élaboration d'un plan d'intervention d'urgence coordonné, intégré et fonctionnant efficacement à l'intention des collectivités locales.

Pour en savoir plus sur APELL, consulter le site http://www.uneptie.org/pc/apell/home.html (en anglais seulement).

\section{Rôle des pays bénéficiaires}

- 16.b.16 Il faudrait que les pouvoirs publics conviennent, lorsqu'ils sollicitent une aide bilatérale ou multilatérale en rapport avec des installations dangereuses, que des lois et des procédures appropriées (comme celles décrites dans les présents Principes directeurs) devraient être en place et appliquées.

" Les pouvoirs publics des pays bénéficiaires devraient prendre toutes les mesures nécessaires pour assurer la coopération avec les fournisseurs de l'aide destinée à améliorer la sécurité des installations dangereuses, par exemple en communiquant les renseignements à l'appui des évaluations et de la mise en œuvre des projets d'aide.

* Tous les pouvoirs publics détenant des responsabilités en matière de sollicitation d'aide ou d'amélioration des mesures de prévention, de préparation et d'intervention applicables aux accidents chimiques devraient coordonner leurs activités pour que l'aide technique et financière soit utilisée aussi efficacement que possible. 
- 16.b.17 Les pouvoirs publics devraient conserver des dossiers de leur expérience à titre de bénéficiaires d'aide bilatérale ou multilatérale en rapport avec des installations dangereuses. Ils devraient partager cette expérience avec les organisations donatrices et avec les autres bénéficiaires d'aide.

16.b.18 Les pouvoirs publics devraient faciliter la diffusion des présents Principes directeurs et de tout autre document d'orientation pertinent à toutes les parties prenantes, y compris les pouvoirs publics à tous les paliers, l'industrie (incluant les travailleurs), les groupes communautaires et les autres organisations concernées. Les pouvoirs publics devraient également prendre l'initiative d'aider ces parties à comprendre et à mettre en œuvre ces documents d'orientation.

\section{c. TRANSFERT DE TECHNOLOGIE ET INVESTISSEMENT INTERNATIONAL}

Dans la présente section, on admet que les pouvoirs publics ne devraient pas exercer de discrimination - en matière de sécurité - entre les installations dangereuses dirigées par des sociétés intérieures et celles où sont engagés des sociétés étrangères, de la technologie importée ou des investissements étrangers.

Il ne s'agit pas ici de traiter de manière exhaustive la question de la circulation de la technologie et des investissements des pays développés vers d'autres pays. L'objectif est plutôt d'illustrer les types d'enjeux dont il faut tenir compte, de même que la nécessité (dans certains cas) de redéfinir les rôles et responsabilités respectifs de l'industrie, des pouvoirs publics et des autres parties pour atteindre le niveau de sécurité voulu.

Bien que les Principes directeurs aient été rédigés dans la perspective de la circulation de technologie ou d'investissements des pays développés vers les pays en développement ou les PET, ils s'appliquent également à d'autres types de transfert de technologie ou d'investissement (par exemple, d'un pays en développement à un autre).

L'application des Principes directeurs devrait faciliter le transfert de technologie et l'investissement puisque les fournisseurs de technologie et les investisseurs potentiels peuvent se montrer réticents à faire affaire avec des pays ou des entreprises bénéficiaires sans connaître leur capacité et leur volonté d'exploiter de façon sûre des installations dangereuses. Les pays ou les entreprises bénéficiaires hésiteront aussi à accepter une technologie qui ne peut être appliquée de manière sûre.

La présente section incorpore des passages traitant des rôles et des responsabilités des pays ou des entreprises bénéficiaires, textes qui ont été élaborés dans le cadre du programme APELL du PNUE.

\section{Principes généraux}

16.c.1 Toutes les parties devraient promouvoir dans les installations dangereuses situées dans tous les pays un niveau de sécurité équivalent à celui atteint dans les installations analogues des pays développés.

- La promotion d'un niveau de sécurité équivalent n'empêche pas les pouvoirs publics ou les entreprises de chercher à atteindre un niveau plus élevé de sécurité.

• La sécurité des installations mises en place grâce à un investissement ou à un transfert de technologie en provenance d'un pays développé devrait se situer au plus haut niveau raisonnablement possible, compte tenu de l'état actuel des connaissances et des circonstances locales. 
" Le transfert de technologie ou l'investissement ne devrait avoir lieu que si on a une certitude raisonnable que des conditions d'exploitation sûres peuvent être réunies.

" Il convient de respecter dans l'installation de bonnes pratiques de conception, d'ingénierie, de construction, d'exploitation et de gestion afin de maintenir en permanence la sécurité. Il faudrait tenir compte aussi de la nécessité d'assurer une sensibilisation et une formation adéquates et de communiquer des informations sur l'installation.

"Les responsabilités, y compris les coûts, liées aux objectifs en matière de sécurité peuvent être attribuées d'un commun accord par les parties concernées.

- 16.c.2 Lorsqu'une entreprise établie dans un pays développé investit dans une installation dangereuse nouvelle d'un pays en développement ou d'un PET, ou qu'elle fournit (transfère) un procédé ou une autre technologie intéressant la sécurité à une telle installation, il importe de choisir le procédé et de concevoir l'installation en tenant compte des conditions locales qui peuvent influer sur la sécurité des installations. Il s'agit, entre autres, des facteurs suivants :

- géographie et climat;

- facteurs culturels et socio-économiques;

- infrastructure, y compris les services d'urgence;

- cadre juridique et administratif;

- politiques d'aménagement du territoire;

- systèmes légaux et de contrôle, locaux;

- disponibilité locale de main-d'œuvre;

- systèmes d'information;

- matériaux de construction et équipement disponibles.

16.c.3 Les fournisseurs de technologie et les investisseurs devraient, en liaison avec les bénéficiaires de la technologie et les pouvoirs publics compétents, procéder à une évaluation des dangers spécifiques au site; celle-ci comprendrait, entre autres, une évaluation des traits culturels et des pratiques propres au pays bénéficiaire qui seraient de nature à justifier une refonte du système technique de sécurité, ainsi qu'une évaluation des répercussions possibles de toute hypothèse de conception pouvant influer sur la sécurité d'utilisation de la technologie sur le site considéré.

" Il pourrait s'agir, par exemple, d'hypothèses relatives à la capacité et à l'importance des services publics d'intervention d'urgence existants, à la fiabilité et à la régularité de l'alimentation électrique, à l'importance de la communauté des ingénieurs de sécurité et à la disponibilité de pièces détachées et d'équipement de maintenance.

" L'évaluation des dangers devrait être utilisée pour décider s'il y a lieu de donner suite au projet de transfert de technologie ou d'investissement.

16.c.4 Toutes les parties associées à un transfert de technologie ou à un investissement devraient s'entendre sur le partage des responsabilités relatives à la construction et à l'exploitation sûre d'une installation dangereuse. L'entreprise bénéficiant de l'investissement ou du transfert de technologie devrait veiller à ce que l'accord procure une capacité adéquate et durable d'exploiter et d'entretenir l'installation de manière sûre, et à disposer des ressources nécessaires (humaines, techniques et financières) pour assumer ses responsabilités conformément à l'entente. 
16.c.5 Les Principes directeurs visant la diffusion de l'information aux employés et au public devraient s'appliquer à toutes les installations dangereuses, où qu'elles se situent, étant entendu toutefois que l'emplacement de l'installation peut influer sur les rôles relatifs de l'industrie et des pouvoirs publics.

" Par exemple, si les pouvoirs publics locaux ne disposent pas de ressources suffisantes pour mettre en œuvre des mécanismes d'information du public, la direction des installations dangereuses devrait veiller à ce que les renseignements pertinents soient mis à la disposition du public, en conformité avec les Principes directeurs.

" Il peut s'avérer impossible d'appliquer telles quelles, dans les autres pays, les méthodes utilisées pour communiquer des renseignements sur les risques dans les pays développés. Pour que l'information transmise soit exacte, complète et comprise, la démarche retenue devrait tenir compte des facteurs locaux comme les structures sociales et familiales, les influences religieuses, les langues et les dialectes, les ressources limitées et les techniques disponibles pour diffuser l'information.

16.c.6 Les organisations internationales doivent continuer à prendre des mesures pour appuyer le principe voulant que le transfert de technologie et l'investissement relatifs à des installations dangereuses ne se fassent que s'ils s'accompagnent du transfert des techniques et du savoir-faire correspondants en matière de sécurité et si l'on a la certitude que des conditions d'exploitation sûres peuvent être réunies dans le pays bénéficiaire.

Transfert de technologie des pays développés vers les pays en développement ou à économie en transition (PET)

Les paragraphes qui suivent portent sur le transfert, par une entreprise établie dans un pays développé, d'un procédé ou d'une autre technologie intéressant la sécurité à une installation dangereuse d'un pays en développement ou d'un PET. Le transfert de technologie peut se faire entre parties indépendantes ou dans le cadre d'une relation entre entreprises. Dans ce dernier cas, la relation peut aller d'une participation minoritaire à la pleine propriété. La nature de cette relation peut influer sur la répartition des responsabilités entre le fournisseur et le bénéficiaire de la technologie, ou sur les moyens de remplir ces responsabilités respectives.

\section{(Voir les paragraphes 2.i.7 à 2.i.10 sur le transfert de technologie en général)}

16.c.7 Les responsabilités de toutes les parties prenantes au transfert de technologie relatif à une installation dangereuse devraient être clairement définies dès les stades préliminaires de la transaction.

" Un contrat écrit entre le fournisseur et le bénéficiaire devrait préciser les obligations de chacun d'eux à l'égard des questions de sécurité touchant la technologie transférée, étant entendu que la responsabilité est liée à la maîtrise effective de l'exploitation.

" Les dispositions retenues devraient tenir compte de la quantité de ressources nécessaires pour se conformer aux exigences en matière de sécurité, ainsi qu'à la politique et aux règles d'orientation de l'entreprise en matière de sécurité. 


\section{- Rôle des fournisseurs de technologie}

16.c.8 Le fournisseur ne devrait exporter que les technologies dont on possède une expérience suffisante pour procéder à des analyses appropriées des dangers qu'elles présenteront sur les lieux où elles seront employées.

16.c.9 Le transfert de technologie relatif à une installation dangereuse ne devrait se faire que s'il s'accompagne du transfert des techniques appropriées de sécurité et de l'information nécessaire à l'exploitation sûre de l'installation.

" Le fournisseur de la technologie devrait communiquer au bénéficiaire de celle-ci et, sur demande, aux pouvoirs publics compétents du pays importateur les renseignements suivants, dans la mesure où ils touchent à la sécurité :

- les réglementations, les prescriptions juridiques et administratives et les pratiques de prévention de son pays en matière d'accidents dans les principaux secteurs d'utilisation de la technologie;

- les normes de sécurité généralement admises, les codes volontaires, les règles des associations professionnelles et les autres documents techniques d'orientation touchant la conception, la mise en place ou l'exploitation de la technologie;

- la description du procédé, y compris toutes les données nécessaires sur les substances mises en jeu, les réactions chimiques qui se produisent, etc.;

- les instructions d'exploitation et les paramètres fondamentaux de fonctionnement dans des conditions normales et inhabituelles;

- une analyse des dangers indiquant, entre autres, les aspects particulièrement dangereux de la technologie, les problèmes de sécurité connus ou soupçonnés associés à la technologie, les possibles produits d'un emballement de la réaction et les effets dominos en cas d'accident, les limites inférieure et supérieure des plages d'exploitation sûre de chaque procédé industriel et les quantités de substances dangereuses, toxiques ou inflammables normalement présentes dans le cadre des opérations ou du stockage;

- toute information complémentaire utile à l'évaluation et à la maitrise des dangers, à l'emploi de la technologie et de la manutention de toutes les substances dangereuses utilisées ou fabriquées en toute sécurité, ainsi qu'à l'examen de la performance en matière de sécurité;

- des directives sur la maintenance, indiquant notamment la fréquence recommandée du contrôle et de la maintenance des constituants essentiels et de l'installation dans son ensemble, des évaluations des coûts prévus de la maintenance, et une description du matériel de contrôle nécessaire ainsi que des compétences requises;

- des manuels et des programmes pour la sensibilisation et la formation des employés.

" Les renseignements ci-dessus devraient être offerts dans une langue appropriée et communiqués aussitôt que possible, au besoin et conformément au contrat avant le transfert effectif de la technologie. Le calendrier de communication des renseignements devrait être convenu au cours du processus de négociation en vue du transfert. 
4 Les dispositions appropriées devraient être prises pour protéger les secrets de fabrication légitimes. Ce qui précède ne diminue en rien les droits de propriété intellectuelle liés au produit ou au procédé qui fait l'objet d'un transfert de technologie.

16.c.10 Le fournisseur de la technologie devrait indiquer au bénéficiaire de celle-ci et, le cas échéant, aux pouvoirs publics du pays importateur si la technologie transférée fait intervenir des activités classées comme dangereuses dans le pays exportateur et (ou), à sa connaissance, dans des pays tiers.

16.c.11 Il incombe au fournisseur de la technologie de concevoir le procédé de façon sûre, de superviser la mise en service, d'assurer la sensibilisation et la formation technique initiales, de fournir une aide au démarrage et de communiquer les informations nécessaires à l'exploitation et à la manutention des produits utilisés et fabriqués en toute sécurité (étant entendu qu'un contrat devrait préciser les devoirs du fournisseur et du bénéficiaire, conformément au paragraphe 16.c.7).

16.c.12 Le fournisseur de la technologie, par l'entremise de son propre personnel ou de consultants, devrait mettre à la disposition de l'entreprise bénéficiaire de la technologie des personnes qualifiées qui puissent prêter leur aide en matière de formation et de sensibilisation au sujet de la sécurité de la technologie; cette aide devrait porter aussi sur l'adaptation de la technologie transférée aux conditions locales et sur son intégration dans l'infrastructure industrielle locale.

" Cette assistance devrait être fournie pendant la conception, la construction, le démarrage et les premiers temps de l'exploitation de l'installation dangereuse.

" Le fournisseur de la technologie peut être tenu de superviser certaines tâches qui incombent normalement au bénéficiaire de la technologie, comme l'ingénierie détaillée, la construction de l'usine, l'application du procédé, la maintenance et les modifications de l'usine, les changements dans la conception ou les méthodes d'exploitation, l'information des autorités locales au sujet des questions de sécurité, la formation et la supervision des travailleurs ainsi que la mise en place de dispositifs de sécurité et de contrôle de celle-ci.

- 16.c.13 Le fournisseur de la technologie devrait, s'il y a lieu, continuer de fournir l'information et l'aide nécessaires à l'exploitation sûre $\mathrm{d}^{\prime} u$ une installation dangereuse après le démarrage, bien que l'étendue et la durée de ces obligations puissent varier selon le type de contrat et son contexte. En tout état de cause, le fournisseur de la technologie devrait communiquer toute information nouvelle intéressant la sécurité qui n'était pas connue au moment du transfert, y compris, par exemple, les renseignements relatifs à un accident ou à un quasi-accident lié à cette technologie.

- Rôle des pays exportateurs

16.c.14 À la demande des pouvoirs publics du pays importateur de la technologie, les pouvoirs publics $\mathrm{du}$ pays exportateur devraient leur communiquer, autant qu'il est raisonnablement possible, les renseignements suivants concernant un transfert prévu ou effectif de technologie relatif à une installation dangereuse : 
- prescriptions juridiques et administratives de même que les réglementations nationales et locales applicables à l'endroit où est implantée et exploitée l'installation;

- information de source gouvernementale concernant les risques et l'utilisation sûre de la technologie transférée ainsi que les fins auxquelles on prévoit l'employer;

- au besoin, études et rapports rendus publics sur les accidents et les incidents déjà survenus.

"Les pouvoirs publics du pays exportateur de la technologie devraient, le cas échéant, être en mesure de recouvrer les coûts de la transmission de ces renseignements auprès du fournisseur de la technologie.

"Il faudrait s'attacher à mettre sur pied un mécanisme international pour la collecte, le traitement et la diffusion de ce type de renseignements à l'échelle mondiale.

Investissement par des entreprises des pays développés dans des installations dangereuses établies dans des pays en développement ou à économie en transition (PET)

Les paragraphes qui suivent portent sur l'investissement international réalisé par une entreprise d'un pays développé dans une installation dangereuse sise dans un pays en développement ou un PET. Cette relation peut s'inscrire dans des contextes divers: soit que l'installation dangereuse se trouve sous la direction effective de l'entreprise établie dans un pays développé (cas des filiales), soit que l'entreprise située dans un pays développé a une participation minoritaire et n'assume pas, par voie contractuelle ou autre, la direction de l'installation dangereuse (on parle alors d'entreprises affiliées). L'investissement peut consister, par exemple, à acquérir une installation existante, à construire une installation nouvelle ou à participer à une coentreprise.

Il convient de noter que nombre des dispositions relatives au transfert de technologie s'appliquent aussi à l'investissement. Celui-ci exige souvent un transfert de technologie, ou encore ce dernier s'impose pour amener l'installation considérée au niveau requis de sécurité.

\section{(Voir les paragraphes 2.i.11 à 2.i.18 sur les acquisitions et activités affiliées en général)}

16.c.15 La prévention des accidents et la garantie de la sécurité devraient figurer parmi les principales préoccupations des entreprises établies dans les pays développés - ainsi que des organisations de services ${ }^{3}$ et institutions financières internationales - lorsqu'elles planifient un investissement relatif à une installation dangereuse d'un pays en développement ou d'un PET. Pour déterminer l'ampleur du financement et de l'assistance dont doit s'accompagner l'investissement, il faudrait tenir compte aussi bien de la quantité de ressources nécessaires pour se conformer aux exigences en matière de sécurité ainsi qu'aux politiques et pratiques de l'entreprise à cet égard, que de l'influence des besoins et des facteurs culturels locaux.

16.c.16 Les investissements des entreprises établies dans les pays développés qui se traduisent par la création d'entreprises nouvelles devraient s'assortir de bonnes pratiques de conception, d'ingénierie, de construction et d'exploitation, afin qu'un niveau élevé de sécurité puisse être maintenu en permanence. Il faudrait prendre en compte les besoins en matière de formation et de transfert d'information afférents à l'installation et à son exploitation dans la localité considérée.

16.c.17 Dans la mesure ou cela s'avère raisonnablement possible, les entreprises établies dans un pays développé devraient s'assurer que leurs filiales appliquent pour la prévention, la 
préparation et l'intervention applicables aux accidents, des politiques et pratiques équivalentes à celles adoptées par ces entreprises dans leur propre pays. La promotion de politiques et pratiques équivalentes n'empêche pas les pouvoirs publics ou les entreprises de chercher à atteindre un niveau plus élevé de sécurité.

" Les moyens retenus pour mettre en œuvre ces politiques et pratiques devraient être adaptés à la situation et aux besoins particuliers de la localité considérée, et prendre en compte les facteurs juridiques, politiques, réglementaires, administratifs, techniques, et autres.

" Les responsables de chaque installation devraient élaborer leurs propres programmes de sécurité pour mettre en œuvre la politique de sécurité de l'entreprise.

" Les employés (y compris les contractants) et les collectivités locales devraient être informés à propos des installations dangereuses et des mesures à prendre en cas $\mathrm{d}^{\prime}$ urgence aussi efficacement que dans le pays où l'entreprise a son siège.

" Les employés devraient disposer, au niveau de la participation aux activités relatives à la sécurité dans les installations dangereuses, de droits équivalents à ceux des employés travaillant dans le pays où l'entreprise a son siège.

- 16.c.18 La politique de sécurité de l'entreprise devrait être diffusée dans la ou les langues nationales du pays au sein des installations dangereuses de toutes ses filiales et, dans la mesure du possible, au sein des installations dangereuses de ses entreprises affiliées.

- 16.c.19 Une entreprise établie dans un pays développé devrait faire en sorte que ses entreprises affiliées adoptent des politiques et pratiques comparables aux siennes en matière de sécurité, et devrait offrir son assistance pour faciliter la réalisation de cet objectif.

16.c.20 Une entreprise établie dans un pays développé qui investit dans des installations dangereuses situées dans un pays en développement ou un PET devrait coopérer avec les autorités locales en vue de s'assurer qu'une infrastructure appropriée existe pour la préparation et l'intervention en cas d'urgence, pour le choix des sites et l'aménagement du territoire et pour la communication d'information au public.

16.c.21 Une entreprise établie dans un pays développé qui exerce une activité dans un pays en développement ou un PET devrait partager avec les entreprises locales de ce pays l'expérience qu'elle a acquise en matière de sécurité (y compris, notamment, l'expérience relative à l'exploitation, à la formation, à la maintenance ainsi qu'à la préparation et à l'intervention en cas d'urgence), étant entendu que les secrets de fabrication doivent être protégés.

16.c.22 Les organisations de services internationales devraient prendre toute mesure raisonnable pour faire en sorte que leurs procédés favorisent l'application de pratiques appropriées en matière de sécurité (en conformité avec les présents Principes directeurs). Il pourrait s'agir pour cela, par exemple, de respecter les principes pertinents dans le cadre de leurs propres activités et de faire connaître les Principes directeurs à ceux de leurs clients privés ou publics qui sont concernés. 


\section{Rôle de l'industrie dans les pays bénéficiaires de la technologie ou de l'investissement}

16.c.23 La direction des installations dangereuses situées dans un pays bénéficiaire devrait faire le nécessaire pour promouvoir un niveau de sécurité équivalent à celui des installations analogues dans les pays développés.

16.c.24 La direction et les autres employés des installations dangereuses devraient faire en sorte que des politiques de sécurité et des systèmes d'exploitation sûrs soient créés et mis en application et, de manière plus générale, ils devraient s'efforcer de remplir leurs rôles et responsabilités tels qu'établis pour les mesures de préparation, de prévention et d'intervention applicables aux cas d'urgence dans les Principes directeurs.

"Dans l'éventualité où les ressources ou les compétences manquent pour assumer ces rôles et responsabilités, ou encore en cas de problème, la direction devrait chercher de l'aide auprès des parties pertinentes; elle peut, par exemple, faire jouer ses relations contractuelles ou solliciter la société mère, le fournisseur de la technologie, une association industrielle, une organisation internationale, etc.

" La présence d'un bassin de travailleurs suffisant pour exploiter l'installation en toute sécurité ainsi qu'une sensibilisation et une formation adéquates permettant d'entretenir en permanence des effectifs qualifiés sont des éléments essentiels. En outre, il est primordial d'établir des systèmes de gestion permettant l'exploitation sûre de l'installation.

" L'industrie dans les pays bénéficiaires devrait faire bon accueil à l'aide offerte par les fournisseurs de technologie, les investisseurs et les fabricants de produits chimiques (à titre d'exemple, par l'entremise des programmes de gérance des produits). L'industrie devrait tâcher par tous les moyens de tirer profit de l'expérience des autres.

16.c.25 La direction des installations dangereuses devrait s'entendre avec tous les employés concernés sur le type d'information que l'on peut et que l'on devrait demander aux fournisseurs de technologie, aux investisseurs et aux organismes d'aide, de même que sur la façon dont cette information devrait être utilisée dans le cadre des processus décisionnels.

"Dans cette perspective, la direction devrait se doter des moyens appropriés pour: faire en sorte que les fournisseurs produisent l'information nécessaire à l'exploitation sûre de l'installation et la maintiennent à jour; savoir quels autres renseignements sont disponibles; se procurer ces renseignements; communiquer l'information à tous les employés concernés.

" La direction des installations dangereuses devrait également mettre en place des mécanismes assurant une utilisation appropriée de l'information, c'est-à-dire son expression en connaissances et en activités, notamment au chapitre de l'établissement de politiques et de procédures pour la sécurité.

" Les bénéficiaires de tels renseignements devraient prendre les dispositions nécessaires pour préserver les secrets de fabrication légitimes.

- 16.c.26 Lorsque les installations en exploitation sont situées dans une collectivité où les pouvoirs publics n'ont pas la capacité d'assumer l'ensemble de leurs rôles et responsabilités, 
l'industrie devrait prendre les mesures supplémentaires qui s'imposent pour assurer la sécurité des installations dangereuses. Le type d'aide qui convient dépendra des conditions locales, mais il peut s'agir de donner de l'information ou des lignes d'orientation, de jouer un rôle accru dans l'élaboration de plans d'urgence externes ou dans la diffusion de l'information au public, ou de procurer de l'équipement d'intervention d'urgence spécialisé. La nature de cette aide devrait être clairement établie.

" Par exemple, la préparation de plans d'urgence externes est une responsabilité qui incombe généralement aux autorités locales. Lorsque ces autorités ne l'assument pas, la direction des installations dangereuses devrait veiller à ce que la préparation soit suffisante pour permettre une intervention adéquate en cas $\mathrm{d}$ 'accident.

" À cet égard, les entreprises d'un même pays ou d'une même région devraient coopérer entre elles.

- 16.c.27 Les entreprises bénéficiaires devraient tout mettre en œuvre pour que des évaluations adéquates des dangers soient effectuées avant la concrétisation de tout investissement, transfert de technologie ou projet d'aide en faveur d'une installation dangereuse.

" Les entreprises des pays bénéficiaires devraient participer activement à de telles évaluations afin de s'assurer que les conditions locales sont pleinement prises en considération, y compris les facteurs culturels, juridiques et environnementaux.

" Les entreprises des pays bénéficiaires devraient mettre l'information relative aux conditions locales (c'est-à-dire l'infrastructure, la main-d'œuvre, le pouvoir réglementaire, la capacité de préparation et d'intervention en cas d'urgence, les populations vulnérables et les milieux écologiquement sensibles) à la disposition des responsables de l'évaluation.

" Dans le même ordre d'idées, les entreprises qui effectuent des évaluations environnementales devraient tenir compte, le cas échéant, des risques d'accident liés à des substances dangereuses et des besoins correspondants en mesures de préparation et d'intervention applicables aux accidents.

16.c.28 La direction et les employés des entreprises bénéficiaires devraient participer aux activités concernant l'adaptation de la technologie aux conditions locales, afin de s'assurer que la technologie, y compris les structures de gestion, convient à ces conditions.

16.c.29 La direction des installations dangereuses dans les pays bénéficiaires devrait établir des procédures pour le maintien de la sécurité dans le temps et pour la gestion des modifications aux installations qui risquent d'influer sur la sécurité. Par exemple, il ne faudrait jamais apporter des modifications avant d'avoir obtenu les informations nécessaires et effectué les évaluations appropriées quant aux implications pour la sécurité. Il faudrait que des programmes de sensibilisation et de formation permanents soient en place pour entretenir les compétences des employés et veiller à ce que les nouveaux employés soient sensibilisés et formés de manière adéquate.

16.c.30 Les entreprises d'un même pays ou d'une même région devraient mettre en place des mécanismes de partage de l'information et de l'expérience au chapitre de la sécurité des 
installations dangereuses, notamment pour tenir compte des questions liées aux paramètres culturels, politiques et environnementaux locaux.

16.c.31 Il faudrait que l'industrie développe l'idée voulant que les organisations professionnelles, les milieux universitaires et les instituts de recherche devraient, au besoin, prêter leur appui à l'évaluation de l'information, à l'élaboration de codes, de normes et de programmes de formation, à la surveillance des installations dangereuses et à l'information du public.

16.c.32 L'industrie, dans les pays bénéficiaires, devrait favoriser la diffusion des présents Principes directeurs à toutes les parties concernées, y compris le personnel à tous les échelons et les organisations n'appartenant pas à l'industrie.

" La direction devrait également s'efforcer d'aider ces parties à comprendre et à mettre en œuvre les Principes directeurs.

"L'industrie devrait appuyer les activités connexes par les pouvoirs publics.

\section{Rôle des pouvoirs publics dans les pays bénéficiaires de la technologie ou de l'investissement}

16.c.33 Les pouvoirs publics des pays bénéficiaires de la technologie ou de l'investissement devraient s'employer à encourager toutes les parties à maintenir, dans toutes les installations situées dans un pays en développement ou un PET, un niveau de sécurité équivalent à celui des installations analogues dans les pays développés. À titre d'exemple, en prévision des propositions de transfert de technologie ou d'investissement de la part d'autres pays, les pouvoirs publics devraient établir des systèmes permettant de s'assurer de la conformité des fournisseurs et des bénéficiaires de la technologie aux pratiques et procédures applicables en matière de sécurité, telles que décrites dans les Principes directeurs.

16.c.34 Les pouvoirs publics des pays bénéficiaires de la technologie ou de l'investissement devraient tout mettre en œuvre pour assumer les rôles et responsabilités qui leur incombent conformément aux Principes directeurs, ce qui comprend notamment le respect des dispositions relatives à la prévention, à l'aménagement du territoire ainsi qu'aux mesures de préparation et d'intervention applicables aux urgences.

" Étant entendu que cela n'est peut-être possible que dans une certaine mesure à cause du caractère limité des ressources, les pouvoirs publics devraient commencer par tracer un cadre législatif et réglementaire à l'appui de la mise en œuvre des Principes directeurs. Les pouvoirs publics devraient établir leurs priorités en fonction de la nature des installations dangereuses présentes sur le territoire sous leur autorité, des problèmes associés à ces installations et de la quantité de ressources disponibles.

" Lorsque les ressources ne permettent pas aux pouvoirs publics d'assumer l'ensemble des rôles et responsabilités qui leur sont assignés, ils devraient faire appel à l'industrie et à d'autres groupes du secteur privé pour que ceux-ci apportent leur soutien aux activités du secteur public et les aident à atteindre les objectifs fixés. Par exemple, dans les pays où l'infrastructure publique est déficiente, l'industrie peut être appelée à participer davantage à la communication de l'information au public ou à la planification pour les situations d'urgence. Dans ce cas, le partage des responsabilités entre les pouvoirs publics et l'industrie devrait être clairement défini. 
" En ce qui concerne un transfert de technologie ou un investissement spécifiques, les pouvoirs publics devraient, au besoin, demander au pays ou à l'entreprise à l'origine du transfert ou de l'investissement, ou encore à des organisations internationales de leur fournir l'information et l'aide nécessaires pour assumer leurs responsabilités. À cette fin, les pouvoirs publics devraient être associés dès que faire se peut au processus de planification concernant le transfert de technologie ou l'investissement.

- 16.c.35 Les pouvoirs publics des pays bénéficiaires de la technologie ou de l'investissement devraient établir quel type d'informations ils peuvent et doivent solliciter, et comment cette information peut être utilisée dans le cadre des processus décisionnels.

1) Dans cette perspective, les pouvoirs publics devraient désigner des responsables de la sollicitation de l'information, de la réception de cette information et de sa diffusion aux parties concernées, y compris les autorités locales.

" Les pouvoirs publics devraient également mettre en place des mécanismes assurant une utilisation appropriée de l'information, tant dans le cadre des décisions relatives à des installations dangereuses particulières que dans celui des processus décisionnels plus généraux, qui englobent les décisions en matière d'aménagement $\mathrm{du}$ territoire, de préparation des plans d'urgence et d'amélioration des infrastructures législatives et réglementaires.

" Les pouvoirs publics devraient prendre les dispositions nécessaires pour préserver les secrets de fabrication légitimes.

16.c.36 Dans la mesure du possible, les pouvoirs publics des pays bénéficiaires de la technologie ou de l'investissement devraient veiller à ce que des évaluations adéquates soient effectuées. À cet égard, ils devraient exiger du fournisseur de la technologie tous les renseignements pertinents.

" Les pouvoirs publics devraient participer activement à l'évaluation de certains projets pouvant entraîner des risques d'accident mettant en cause des substances dangereuses ou d'accroître les risques existants (projets concernant, par exemple, le transfert de technologie, un investissement ou de l'aide en rapport avec une nouvelle installation, ou avec des modifications en profondeur à une installation existante), de telle façon que les conditions locales soient pleinement prises en compte, notamment les facteurs culturels, juridiques et environnementaux.

" Les pouvoirs publics devraient fournir aux responsables de l'évaluation l'information qu'ils possèdent sur les conditions locales pertinentes (par exemple, l'infrastructure, la main-d'œuvre, le pouvoir réglementaire, la capacité de préparation et d'intervention applicables aux urgences, les populations vulnérables et les milieux écologiquement sensibles).

" Les pouvoirs publics devraient également s'assurer que l'on tient compte dans le cadre des évaluations environnementales, sil y a lieu, des risques d'accident liés à des substances dangereuses et des besoins correspondants en capacité de préparation et d'intervention applicable aux urgences.

16.c.37 Les pouvoirs publics des pays bénéficiaires de la technologie ou de l'investissement devraient concevoir des activités liées à l'adaptation de la technologie aux conditions 
locales et, le cas échéant, y participer, pour s'assurer que la technologie, y compris les structures de gestion, convient à ces conditions.

16.c.38 Lorsque les pouvoirs publics sont partie à des ententes sur le partage des responsabilités concernant un transfert de technologie ou un investissement, ils devraient s'assurer de disposer des ressources humaines, financières et techniques nécessaires pour assumer les responsabilités contractées.

16.c.39 Les pouvoirs publics des pays bénéficiaires de la technologie ou de l'investissement devraient établir et appliquer le principe voulant que les installations dangereuses soient capables de maintenir un niveau adéquat de sécurité sur une base permanente.

" Il faudrait éviter d'apporter des modifications importantes aux installations dangereuses existantes ou aux ententes sur l'exploitation de telles installations sans avoir pris en considération les questions de sécurité. Normalement, la direction des installations est responsable de procéder à l'évaluation de la sécurité et de veiller à ce que l'information et le personnel qualifié adéquats soient disponibles pour mener à bien cette évaluation.

" Étant entendu que la sensibilisation et la formation des membres de la direction et des employés sont d'abord et avant tout la responsabilité de l'industrie, les pouvoirs publics devraient prendre les mesures appropriées pour faciliter, globalement, le maintien de travailleurs dans l'industrie et de représentants au sein des pouvoirs publics, qui sont formés et sensibilisés aux questions de sécurité.

- 16.c.40 Dans la mesure du possible, les pouvoirs publics devraient établir des procédures de contrôle des technologies importées et des investissements étrangers qui sont susceptibles d'accroître de manière significative les risques d'accident liés à des substances dangereuses. Ces procédures devraient aider à faire en sorte que la technologie ou l'investissement reçus s'accompagnent de l'information ainsi que des mesures de sensibilisation et de formation requises.

16.c.41 Les pouvoirs publics devraient essayer de s'assurer qu'une capacité d'intervention suffisante existe en cas d'accident lié à une installation dangereuse. Il est entendu que nombre de collectivités locales ne disposent pas du personnel, de l'équipement et des installations qu'il faudrait pour intervenir en cas d'urgence. Cependant, ce problème pourrait être résolu, par exemple, en octroyant des ressources à l'échelle régionale et nationale, en obligeant l'industrie à combler les lacunes en matière de personnel, d'équipement et d'installations (par l'intermédiaire des réseaux d'entraide existant au sein de l'industrie), ou en prenant part à des accords de coopération avec les collectivités voisines. 


\section{Chapitre 17}

\section{INSTALLATIONS FIXES ET TRANSPORT}

Le présent chapitre concerne certains aspects du transport des substances dangereuses (marchandises dangereuses). De manière plus précise, on y propose des lignes d'orientation intéressant le transport dans la mesure où il fait appel à des installations fixes, ce qui comprend:

- les interfaces des transports en général (gares de triage, gares routières, aéroports, installations de chargement et de déchargement);

- les zones portuaires;

- les pipelines.

(Voir l'encadré ci-après pour des exemples de ce qui différencie les interfaces des transports des autres installations fixes aux fins des mesures de prévention, de préparation et d'intervention applicables aux accidents chimiques)

Il importe de savoir que le présent chapitre est un complément au reste des Principes directeurs. Autrement dit, bien que les dispositions contenues dans les autres sections de ces principes s'appliquent aux interfaces des transports et à d'autres aspects du transport mettant en jeu des installations fixes, le chapitre 17 présente d'autres lignes d'orientation propres aux interfaces des transports (y compris les zones portuaires) et aux pipelines.

De plus, ce chapitre présente des lignes d'orientation quant aux rôles et responsabilités des parties concernées, qui comprennent ici, en plus des parties prenantes visées de manière générale par les Principes directeurs, les propriétaires et les exploitants d'interfaces des transports et de pipelines, les propriétaires et les exploitants de moyens de transport (navires, camions, trains) et les travailleurs participant au transport et aux manœuvres de chargement ou de déchargement.

Dans ce chapitre, on présuppose que l'identification adéquate des dangers associés aux substances dangereuses et le confinement, le conditionnement, l'emballage, la séparation du fret, l'arrimage, le marquage, l'étiquetage, la pose de plaques et la documentation appropriés sont des conditions indispensables pour un transport et une manutention sûrs des substances dangereuses.

Chaque pays ou instance devrait décider dans quelle mesure les substances sont assujetties à la réglementation sur le transport et, dans quelle mesure, elles sont soumises à d'autres exigences (par exemple, celles qui s'appliquent à l'entreposage ou aux installations fixes en général). L'attribution des responsabilités peut varier d'un pays à l'autre, mais en aucun cas il ne doit y avoir des lacunes dans la réglementation.

\section{a. INTERFACES DES TRANSPORTS 4}

17.a.1 Les limites géographiques des interfaces des transports où sont manipulées des substances dangereuses doivent être clairement définies et devraient englober les zones de manutention, de transport et (ou) d'entreposage temporaire des substances dangereuses.

- Les zones où l'on garde des substances dangereuses devraient être clairement indiquées comme telles, et faire l'objet d'une surveillance adéquate ainsi que $\mathrm{d}$ 'inspections régulières pour y détecter d'éventuels fuites ou dommages. 
" Les dispositions relatives à l'aménagement du territoire devraient être appliquées aux interfaces des transports de telle façon que leur emplacement permette de réduire au minimum les risques d'effets nuisibles en cas d'accident, et de manière à prévenir tout développement inapproprié à proximité des interfaces.

" Au moment de planifier et de construire de nouvelles installations ou d'agrandir des installations existantes dans les interfaces des transports, il faudrait tenir compte des exigences en mesures de prévention et d'intervention applicables à des accidents mettant en cause des substances dangereuses. Cela suppose une évaluation des risques en vue de déterminer la probabilité d'accidents et leurs effets potentiels sur la santé et l'environnement, ainsi que la prévision des dispositifs et de l'équipement de sécurité qui s'imposent.

" Des dispositions permettant de maintenir la sécurité aux interfaces des transports où l'on trouve des substances dangereuses devraient être en place de manière à réduire au minimum toute menace potentielle pour la sécurité, comme celle représentée par des activités terroristes, du sabotage, du vandalisme ou le vol de ces substances.

\section{CARACTÉRISQUES PERTINENTES DES INTERFACES DES TRANSPORTS}

$\mathrm{Au}$ chapitre de la prévention des accidents chimiques ainsi que des mesures de préparation et $\mathrm{d}$ 'intervention applicables à ces accidents, un certain nombre de caractéristiques distinguent les interfaces des transports des installations fixes, et notamment les suivantes:

- différents modes de transport se croisent aux interfaces, chacun ayant ses propres systèmes de contrôle et peut-être même ses pratiques particulières en matière de sécurité;

- la quantité et la nature des substances dangereuses présentes aux interfaces, en vrac ou emballées, sont variables;

- des opérations de transfert et de manutention s'y déroulent en permanence;

- les étapes du conditionnement, de l'étiquetage et de la documentation peuvent se situer loin des interfaces, donc hors du contrôle des responsables de la sécurité de celles-ci;

- les parties concernées sont différentes de celles qui interviennent dans les installations fixes, et plus nombreuses. Par exemple, «l'exploitant» ou le "gestionnaire» de l'interface des transports peut appartenir au secteur privé ou public; les travailleurs de l'interface comprennent ceux qui sont aux commandes des divers moyens de transport (chauffeurs, pilotes et capitaines), ceux qui sont responsables du chargement et du déchargement (manutentionnaires), et d'autres encore qui peuvent être employés par différentes sociétés. Parmi les autres parties jouant un rôle crucial au niveau des mesures de prévention, de préparation et d'intervention applicables aux accidents chimiques figurent: les transporteurs, les expéditeurs et les consignateurs de substances dangereuses; les responsables du conditionnement et de l'étiquetage; enfin, les clients. Dans les zones portuaires, un certain nombre d'intervenants s'ajoutent à la liste.

17.a.2 Les diverses parties associées à la manutention des substances dangereuses aux interfaces des transports devraient coopérer de manière à permettre une exploitation sûre de celles-ci et à répondre aux besoins en matière de préparation et d'intervention. Ces parties comprennent les exploitants des interfaces des transports, les transporteurs (tous moyens de transport transitant par les interfaces confondus), les chargeurs 5 , les clients, les pouvoirs publics et d'autres encore. 
17.a.3 Toutes les parties engagées dans le transport des substances dangereuses devraient s'assurer d'avoir accès à l'information nécessaire pour assumer leurs responsabilités concernant la manutention sûre des cargaisons qui renferment des substances dangereuses et pour fournir aux autres parties les renseignements sur ces substances.

" Les responsables de l'expédition, de l'emballage, du conditionnement, du reconditionnement, du marquage, de l'arrimage, de l'étiquetage, de la pose de plaques et de la documentation concernant les substances dangereuses aux installations dangereuses devraient s'assurer que toutes les informations pertinentes sont transmises à leurs successeurs dans la chaîne du transport. Ces informations devraient permettre de retracer les cargaisons renfermant des substances dangereuses et devraient porter sur les substances manutentionnées et donner des lignes d'orientation pour une manutention sûre, pour la préparation aux situations $\mathrm{d}^{\prime}$ urgence et pour l'intervention en cas d'incident.

" Les lignes d'orientation devraient être présentées sous une forme et dans une langue qui puissent être comprises par les personnes qui pourraient être appelées à réagir à une situation d'urgence, notamment les chauffeurs, les pilotes, les capitaines et le personnel d'intervention.

- 17.a.4 Toutes les parties oeuvrant dans la chaîne du transport devraient s'assurer que leurs employés (y compris les contractants) possèdent les compétences et la formation requises pour la manutention des substances dangereuses en conditions tant normales qu'inhabituelles.

17.a.5 Les exploitants ou les gestionnaires devraient rédiger des « rapports sur la sécurité » aux interfaces des transports où il existe des risques d'accident grave lié à des substances dangereuses, ces documents devant être adaptés selon le niveau de risque propre à chaque site.

\section{(Voir les paragraphes 2.a.16 à 2.a.18 traitant des rapports sur la sécurité)}

17.a.6 Les exploitants ou les gestionnaires devraient élaborer et mettre en application un système et des procédures de gestion de la sécurité qui soient propices à la manutention sûre des substances dangereuses à leur interface des transports. Ce système devrait viser tous les moyens de transport transitant à l'interface, et non pas uniquement les principaux d'entre eux (par exemple, les exploitants de gares de triage devraient se préoccuper aussi des camions livrant des substances dangereuses à leur interface).

\section{(Voir les paragraphes 2.a.14 à 2.a.15 sur les systèmes de gestion de la sécurité)}

17.a.7 Les exploitants ou les gestionnaires devraient veiller à ce que l'équipement et les systèmes de sécurité (y compris le matériel informatique et les logiciels) utilisés à l'interface des transports conviennent à l'emploi qui en est fait et soient conformes aux normes techniques actuelles.

1) L'un des risques les plus fréquents aux interfaces des transports se situe au niveau des manœuvres de chargement et de déchargement. Il faudrait porter une attention particulière au matériel utilisé à ces fins, notamment les grues, les pompes, la tuyauterie souple et les pipelines, de même que les instruments de contrôle de l'équipement, les indicateurs de trop-plein automatiques et les dispositifs de fermeture automatique. 
" Les exploitants ou les gestionnaires des interfaces des transports devraient veiller à ce que l'ensemble des systèmes et de l'équipement de sécurité utilisés dans le cadre des manœuvres de chargement et de déchargement ou de la manutention générale des substances dangereuses fassent l'objet d'une maintenance et d'un contrôle appropriés. À cet égard, il faut garder à l'esprit que l'équipement et les systèmes peuvent appartenir à différents contractants.

" Les exploitants ou les gestionnaires devraient s'assurer que l'équipement et les systèmes de sécurité sont conçus et employés de manière à réduire au minimum les risques d'erreur humaine, et que les employés sont formés pour utiliser l'équipement et les systèmes en toute sécurité (sachant qu'il y a souvent des contractants ou des travailleurs à court terme employés aux interfaces des transports).

" Les exploitants ou les gestionnaires devraient chercher à remplacer les technologies désuètes lorsqu'il existe des solutions de remplacement plus sûres.

17.a.8 Les exploitants ou les gestionnaires devraient également:

- s'assurer qu'ils possèdent l'information nécessaire pour que les substances dangereuses soient manutentionnées en toute sécurité et, à cet égard, qu'ils disposent de réseaux leur permettant d'être avisés d'avance de l'arrivée ou du départ de substances dangereuses destinées à transiter par l'interface des transports, à y être manutentionnées ou à y séjourner temporairement;

- conserver un registre des substances dangereuses arrivant à l'interface des transports, indiquant leur quantité, leur classe et leur emplacement;

- mettre en place des mécanismes permettant de vérifier si tous les contractants concernés ont les compétences nécessaires pour accomplir le travail prévu, et refuser de passer un contrat si ce dernier peut compromettre la sécurité;

- établir des méthodes pour sélectionner et réévaluer les aptitudes des transporteurs et l'équipement que l'on se propose d'employer;

- prévoir des procédures pour les cargaisons endommagées renfermant des substances dangereuses;

- avoir le droit de refuser une cargaison si l'on considère qu'elle représente une menace pour la santé ou l'environnement, y compris les biens.

17.a.9 Les chargeurs (incluant les fabricants, les expéditeurs ou les consignateurs, les transitaires, les groupeurs, les emballeurs, les courtiers et les négociants) devraient :

- veiller à ce que l'information nécessaire à la manutention sûre des substances dangereuses ainsi qu'à la préparation et à l'intervention applicables aux urgences soit à la disposition des gestionnaires des interfaces des transports et, au besoin, des pouvoirs publics;

- définir des méthodes pour sélectionner et réévaluer la compétence des transporteurs et l'équipement que l'on se propose d'employer.

- 17.a.10 Les transporteurs devraient :

- garder un inventaire des produits transportés;

- veiller au choix et à la maintenance de l'équipement approprié;

- veiller à ce que tous les documents soient transmis comme il se doit à la partie responsable qui prend le relais dans la chaîne du transport, et à ce que les documents appropriés accompagnent la prise en charge des substances dangereuses lors de leur chargement, de leur déchargement ou de leur transfert. 
17.a.11 Les clients (à l'interface des transports au point de livraison) devraient :

- s'assurer qu'ils ont en main toutes les informations nécessaires à la manutention des substances dangereuses en toute sécurité ainsi qu'à la préparation et à l'intervention applicables aux urgences;

- prévoir les procédures de même que l'équipement et les installations requises pour remédier aux problèmes liés à des fuites et à des conteneurs endommagés, et enfin pour recueillir les substances et les transporter vers des sites d'entreposage sûrs aussi rapidement que possible.

17.a.12 Il faudrait être spécialement vigilant en ce qui concerne l'entreposage des substances dangereuses aux interfaces des transports.

" À cet effet, la réglementation visant l'entreposage des substances dangereuses devrait s'appliquer à l'entreposage de ces substances aux interfaces des transports.

" L'ampleur de l'entreposage des substances dangereuses aux interfaces des transports (exprimée en quantité de substances, en nature des dangers connexes ou en durée d'entreposage) devrait être réduite à un minimum correspondant à un niveau élevé de sécurité (afin de diminuer la probabilité globale d'accidents liés à des substances dangereuses et les conséquences de tels accidents).

- 17.a.13 Les pouvoirs publics devraient s'assurer que leur cadre de contrôle et leurs activités d'application de la loi (y compris la surveillance et l'inspection) couvrent les interfaces des transports. Ce cadre de contrôle devrait notamment :

- permettre de vérifier si les gestionnaires et les transporteurs sont aptes à assurer la manutention en toute sécurité des substances dangereuses qui transiteront par les interfaces des transports;

- définir les classes et les quantités de substances dangereuses qu'il est autorisé de manutentionner et de faire transiter par les interfaces des transports, et les conditions de manutention à respecter.

- 17.a.14 Les interfaces des transports où transitent des substances dangereuses devraient être munies de plans d'intervention d'urgence bien coordonnés avec les plans d'urgence externes et autres plans pertinents.

" Les plans d'urgence devraient permettre au personnel d'intervention d'accéder facilement aux lieux en cas d'accident.

" Le matériel et l'équipement d'intervention requis selon les plans d'urgence interne et externe devraient se trouver à l'interface des transports.

" Il devrait exister un inventaire des substances sur place aux fins de la planification pour les situations d'urgence et en vue de faciliter les mesures d'intervention en cas d'accident.

17.a.15 Les exploitants ou les gestionnaires ainsi que les pouvoirs publics devraient conjuguer leurs efforts afin de garantir que l'information au sujet des dangers potentiels et des mesures à prendre en cas d'accident soit transmise en continu aux populations susceptibles d'être touchées. 
" Étant donné que les interfaces des transports constituent un défi significatif à cet égard, des formules inédites pourraient être nécessaires pour s'assurer que le public est bien informé.

" Les pouvoirs publics et l'industrie devraient s'employer à partager leurs informations et leur expérience concernant la communication avec le public au sujet des interfaces des transports.

17.a.16 Il devrait exister des circuits permettant la notification/déclaration rapide des incidents (accidents et quasi-accidents) survenus aux interfaces des transports.

" Plus précisément, les chargeurs, les transporteurs et les clients devraient aviser les exploitants ou les gestionnaires de l'interface en cas d'incident lié à des substances dangereuses (c'est-à-dire, fuites ou conteneurs endommagés) et, s'il y a lieu, en informer les pouvoirs publics (y compris le personnel d'intervention) et les fabricants des substances.

" Il faudrait redoubler d'efforts pour partager, à l'échelle tant nationale qu'internationale, l'expérience relative aux incidents survenus à des interfaces des transports.

- 17.a.17 À l'échelle nationale, les pouvoirs publics devraient adopter une attitude cohérente, du point de vue légal et politique - incluant des mécanismes de supervision et de coordination -, à l'égard de tous les moyens de transport. Cela aide à éliminer les déficiences et les incohérences dans les exigences réglementaires ou dans l'attribution des responsabilités tout au long des transferts de substances dangereuses d'un moyen d'un transport à un autre.

- 17.a.18 Il faudrait harmoniser davantage les lois et les politiques qui concernent les interfaces des transports 6 .

" L'adoption de lignes de conduite harmonisées dans les différents pays contribue à uniformiser les «règles du jeu», ce qui signifie qu'aucun pays ne peut prétendre offrir à l'industrie un avantage économique en raison de normes de sécurité moins sévères qu'ailleurs.

" L'harmonisation des lois et politiques empêche également la mise en place de barrières commerciales et entraîne une réduction globale des coûts puisque les entreprises dont les activités se déroulent dans divers pays ou régions n'ont pas à se conformer à différentes normes. Cela revêt une importance particulière dans le secteur des transports, où des substances dangereuses peuvent franchir les frontières.

- 17.a.19 Les pouvoirs publics devraient également poursuivre leur collaboration en ce qui concerne les efforts d'harmonisation des exigences internationales applicables aux divers moyens de transport. Étant donné la diversité des moyens de transport qui se rencontrent aux interfaces des transports (navires, barges fluviales, trains, camions, pipelines), une certaine cohérence devrait se retrouver parmi les différents règlements, règles et politiques ayant trait au transport, au conditionnement et à la manutention des substances dangereuses. Les Recommandations du Comité d'experts des Nations Unies en matière de transport des marchandises dangereuses constituent la base pour 
l'harmonisation des exigences applicables aux divers moyens de transport de marchandises sous emballage.

17.a.20 La direction des installations dangereuses devrait s'efforcer de choisir le moyen de transport et l'itinéraire les plus sûrs pour acheminer les substances dangereuses à partir des installations ou vers celles-ci. Cela contribuera, par exemple, à réduire le nombre de personnes susceptibles d'être touchées en cas d'accident.

- Les évaluations des risques devraient être l'une des données utilisées dans les processus décisionnels pour comparer les différents moyens de transport et les divers itinéraires possibles pour acheminer les marchandises dangereuses.

(Voir la section 2.b sur la caractérisation des dangers et l'évaluation des risques)

- Le choix d'un moyen de transport devrait se faire au cas par cas, puisque les études montrent qu'aucun moyen de transport n'est invariablement plus sûr qu'un autre. La sécurité dépend d'un certain nombre de facteurs, notamment la substance en cause, l'itinéraire retenu et les pratiques de gestion locales.

- Dans la mesure où la direction des installations dangereuses peut choisir entre divers moyens de transport et différents itinéraires pour acheminer les substances dangereuses, les décisions qu'elle prend devraient tenir compte des questions plus générales intéressant l'environnement et la santé.

- La direction des installations dangereuses devrait s'assurer que les transporteurs de ses produits respectent les exigences nationales et internationales en matière de sécurité. Dans la mesure du possible, il faudrait sélectionner les transporteurs en se fondant sur leurs antécédents en matière de sécurité.

- La direction devrait coopérer avec les pouvoirs publics (y compris les autorités locales) lorsqu'elle prend des décisions relatives au transport de substances dangereuses et à l'itinéraire emprunté par celles-ci.

17.a.21 Il faudrait s'efforcer d'améliorer la collecte de l'information sur l'ampleur et la nature du transport de substances dangereuses ainsi que la diffusion de cette information aux parties concernées.

\section{b. ZONES PORTUAIRES}

La présente section met l'accent sur les questions spécifiquement liées aux zones portuaires ${ }^{7}$. Les zones portuaires forment un sous-ensemble des interfaces des transports et, en conséquence, toutes les dispositions des présents Principes directeurs (y compris la section 17.a ci-dessus, qui a trait aux interfaces des transports) s'appliquent à ces zones. Étant donné que les zones portuaires, par rapport aux autres interfaces des transports, possèdent des particularités additionnelles et engagent un plus grand nombre de parties prenantes, il convient de donner des lignes d'orientation supplémentaires à leur sujet. Parmi les caractéristiques propres aux zones portuaires figurent les suivantes :

- Les ports ont intrinsèquement un caractère international; ils rassemblent des exploitants, des navires et des cargaisons en provenance de divers pays.

- Les ports forment des ensembles vastes et complexes où se croisent trafic maritime et transport intérieur (par voie fluviale, ferroviaire et routière) de substances dangereuses. Ils peuvent comprendre un certain nombre d'installations fixes, notamment les gares, les entrepôts, les 
installations de réparation et la maintenance, où des substances dangereuses sont transférées, utilisées, manutentionnées ou entreposées.

- La complexité des zones portuaires complique les décisions d'aménagement du territoire concernant la construction, tant à l'intérieur qu'à l'extérieur de la zone portuaire.

- Pour des raisons historiques, les ports sont généralement situés à proximité de grandes régions à forte densité de population. De plus, d'une façon générale, les secteurs riverains attirent les constructions résidentielles et autres aménagements.

- L'interface navire-terre entraîne le risque, en matière d'exploitation, d'un conflit d'intérêts entre la protection de l'environnement et la sécurité maritime.

- En plus des parties prenantes qui sont associées aux autres interfaces des transports, on trouve aussi dans les zones portuaires d'autres intervenants, comme les autorités portuaires, les agents maritimes, l'administration de l'État du pavillon des navires fréquentant le port, les responsables des postes d'amarrage ainsi que les agents d'inspection des navires et des cargaisons.

(La présente section contient des passages de la publication conjointe de l'OCDE et de l'OMI intitulée Directives relatives à la sécurité des substances chimiques dans les zones portuaires (1996), que l'on peut obtenir auprès de l'OCDE ou trouver à l'adresse wwww.oecd.org/ehs.)

17.b.1 Les autorités portuaires devraient établir, en conformité avec les lois et règlements applicables, des règles portuaires locales relatives à la sécurité des substances dangereuses dans les zones portuaires, et veiller à leur observation.

" Tous les exploitants, dans une zone portuaire, devraient se coordonner avec les autorités portuaires et avec les pouvoirs publics compétents afin de faire en sorte que les activités de chacun n'accroissent pas les risques d'accident (par effet domino) et afin de faciliter la planification et l'intervention en cas d'urgence.

" Les autorités portuaires doivent être au courant des activités de chacun des exploitants dans la zone portuaire et assurer la coordination et des échanges appropriés avec les pouvoirs publics.

- 17.b.2 Les autorités portuaires devraient veiller à ce que tous les usagers de leur port (comme les responsables des postes d'amarrage) mettent au point des procédures d'exploitation pour les manœuvres et les activités qui peuvent influer sur les risques d'accident lié à des substances dangereuses.

17.b.3 Une instance internationale devrait fixer des paramètres garantissant que les navires entrant et manœuvrant dans les ports le font en toute sécurité, paramètres qui peuvent être adaptés selon les circonstances, d'un port à l'autre.

17.b.4 Il faudrait mettre en place un réseau international de déclaration des défectuosités des navires qui influent sur les risques d'accident, et de diffusion de ces rapports aux autorités portuaires.

17.b.5 Les autorités portuaires devraient établir des procédures adéquates pour l'exécution des travaux de réparation et de maintenance des navires transportant des substances dangereuses.

17.b.6 Avant d'entrer dans une zone portuaire, le capitaine d'un navire transportant des substances dangereuses devrait vérifier que l'état matériel du navire et de la cargaison permet d'entrer au port et de procéder aux opérations de manutention de la cargaison en toute sécurité. 
" Le capitaine devrait informer les autorités portuaires de toute défectuosité du navire, de ses machines, de son armement ou de ses engins ou de toute fuite de substances dangereuses ou encore de tout dommage à leurs systèmes de confinement susceptible d'engendrer des risques d'accident lié à ces substances.

"Le capitaine devrait s'assurer que, lorsque son navire entre dans la zone portuaire, toutes les exigences en matière de sécurité, y compris celles qui ont trait à l'arrimage, au conditionnement et à la séparation des substances dangereuses, sont scrupuleusement respectées.

17.b.7 Les responsables des postes d'amarrage devraient s'assurer que des dispositifs d'amarrage adéquats et sûrs sont disponibles et qu'un accès en toute sécurité est prévu entre le navire et la terre.

" Les responsables des postes d'amarrage devraient s'assurer qu'une liste de toutes les substances dangereuses se trouvant dans les installations, accompagnées de leur emplacement et des informations relatives à la sécurité, puisse être consultée facilement.

" Les responsables des postes d'amarrage devraient s'assurer que les substances dangereuses qui pénètrent dans le périmètre du poste d'amarrage ont été dûment certifiées ou déclarées par les chargeurs concernés comme étant convenablement identifiées, emballées, marquées, étiquetées ou placardées.

" Les responsables des postes d'amarrage devraient veiller à ce que personne, sans raison valable, n'ouvre un conteneur, une citerne ou un véhicule qui renferme des substances dangereuses, ou n'intervienne d'aucune autre manière sur ceux-ci.

17.b.8 Les responsables des postes d'amarrage devraient assurer la coordination entre les capitaines de navire et les responsables des autres modes de transport afin de s'assurer que le transfert des cargaisons et l'arrimage des substances dangereuses sont effectués correctement suivant tous les règlements et codes pertinents.

- 17.b.9 Les chargeurs devraient s'assurer que les conteneurs, citernes et véhicules utilisés pour le transport de substances dangereuses ont été agréés du point de vue de la sécurité par une autorité compétente, et que cet agrément est valide. Les chargeurs devraient s'assurer que l'on procède à un examen externe de chaque conteneur, conteneur-citerne, citerne mobile ou véhicule pour vérifier qu'il n'a pas subi de détérioration évidente pouvant avoir une incidence sur sa sécurité.

- 17.b.10 Les chargeurs et les responsables des postes d'amarrage devraient s'assurer que toute l'assistance nécessaire est fournie aux autorités portuaires ou à toute autre personne ou agence habilitée à procéder à des inspections ou vérifications.

- 17.b.11 Les pouvoirs publics devraient veiller à ce que tous les plans d'urgence visant la zone portuaire soient compatibles les uns avec les autres et qu'ils soient tous soumis à un contrôle opérationnel par la partie ou l'autorité désignée.

" Les plans d'urgence devraient tenir compte du fait que l'exploitation des ports engage généralement un grand nombre d'organismes publics et privés. 
" Afin de garantir la compatibilité des démarches et l'uniformité des termes employés, les personnes chargées de l'élaboration des plans d'urgence dans les ports devraient, si possible, avoir recours à des méthodes reconnues et acceptées à l'échelle internationale.

17.b.12 Les capitaines de navire devraient être informés de l'organisation des services d'intervention d'urgence au port et du rôle de leur navire et de leur équipage dans ce système. Les autorités portuaires devraient être informées du plan d'intervention du navire de manière que les actions puissent être coordonnées. À chaque site de transfert de cargaison, le capitaine du navire et le responsable du poste d'amarrage devraient se mettre d'accord sur les procédures d'urgence appropriées.

17.b.13 Les plans d'urgence des ports devraient tenir compte du fait que des substances dangereuses peuvent être transportées dans la zone portuaire par des navires et d'autres modes de transport, même s'il n'est pas prévu de charger ou de décharger ces substances dans la zone portuaire. Les plans d'urgence devraient également prendre en considération la possibilité de situations d'urgence à bord des navires mettant en cause des substances dangereuses qui représentent une menace pour le port ou le milieu marin.

17.b.14 Les services d'intervention d'urgence du port devraient être disponibles et prêts à intervenir dans le cas où un accident se produirait, où que ce soit dans la zone portuaire. À cet égard, les services d'intervention devraient être en mesure de mener et de soutenir avec efficacité les manœuvres s'effectuant du quai au navire, sur le quai, à terre et de navire à navire.

\section{c. PIPELINES}

Bien que les dispositions de l'ensemble des présents Principes directeurs s'appliquent, de manière générale, aux pipelines, la section qui suit traite des questions propres aux pipelines qui transportent des substances dangereuses. Aux fins du présent document, la définition de "pipeline »8 inclut les installations auxiliaires telles que les stations de pompage et de compression.

On apprécie de mieux en mieux la valeur des pipelines comme moyen de transporter toute une gamme de substances dangereuses autres que les produits pétrochimiques. L'expérience montre que les pipelines sont généralement sûrs et qu'ils constituent, dans le cas de certaines substances, un moyen de transport vital. Les pipelines ont entres autres l'avantage de permettre le transport rapide de grandes quantités de substances dangereuses, à relativement peu de frais et de manière assez fiable, avec assez peu d'impact sur l'environnement (en comparaison des autres moyens de transport, qui produisent des gaz d'échappement ou du bruit, encombrent les voies de circulation ou sont déplorables d'un point de vue esthétique).

Parmi les désavantages des pipelines, on compte les coûts d'infrastructure associés à leur construction, les délais inévitables avant leur mise en service, les problèmes relatifs à la protection des sols, et le manque de souplesse quant aux points de livraison et aux quantités pouvant être transportées.

La réglementation, en ce qui concerne les pipelines, varie de manière considérable d'un pays à l'autre, bien qu'il y ait des éléments communs à la plupart des principes réglementaires (notamment l'obligation d'exploiter le pipeline en toute sécurité). En dépit de ces différences, l'industrie des divers pays semble exercer des pratiques de sécurité analogues en vue de préserver l'intégrité des réseaux de pipelines. 
- 17.c.1 Les pipelines destinés au transport des substances dangereuses devraient être conçus, construits, exploités, entretenus et contrôlés de manière à réduire la fréquence des accidents et à atténuer les conséquences de ceux-ci quand il s'en produit.

" Les pipelines devraient être conçus, construits et exploités en conformité avec les codes, les normes et les règles d'orientation reconnus à l'échelle nationale et internationale de même qu'avec les spécifications industrielles.

4I Il faudrait prendre en considération les divers aspects pouvant influer sur la sécurité du pipeline, entre autres les facteurs de conception et de contrainte, la qualité des matériaux, l'épaisseur des parois, la profondeur d'enfouissement, la protection externe contre les chocs, le balisage, le choix du tracé et le contrôle.

"Des évaluations comparatives des risques devraient être effectuées afin de choisir un matériau ou une solution donnés parmi différentes possibilités.

" Pour la construction des pipelines, il faudrait employer les matériaux les plus aptes à garantir l'intégrité des structures dès le départ et pendant l'ensemble de leur cycle de vie. Il faudrait employer des technologies appropriées en matière de sécurité, par exemple des dispositifs de fermeture automatique (en cas de fuite ou d'accident) ou des dispositifs de déclenchement de sécurité.

" Une signalisation de sécurité adéquate devrait être installée le long du tracé du pipeline.

17.c.2 Les questions relatives à l'aménagement du territoire et les évaluations des risques devraient être prises en compte aussi bien pour le tracé des nouveaux pipelines (en évitant autant que possible la proximité avec des zones peuplées) que lors de la prise de décisions concernant des projets de nouveaux aménagements ou de nouvelles constructions au voisinage de pipelines existants.

" L'évaluation environnementale relative aux risques géologiques devrait également être prise en compte afin d'éviter (dans la mesure du possible) les milieux géologiques à risque, par exemple les zones propices à la formation de gouffres et à l'activité sismique.

" Le tracé des pipelines devrait être choisi de manière à réduire le plus possible les répercussions nuisibles d'un éventuel accident, et à en faciliter l'accès aux responsables de la maintenance et du personnel d'intervention d'urgence.

- 17.c.3 L'industrie devrait élaborer des systèmes de gestion de la sécurité afin d'atteindre les objectifs fixés en matière de sécurité pendant la conception, la construction, l'exploitation, la maintenance et la mise hors service des pipelines.

" Les systèmes de gestion de la sécurité concernant les pipelines doivent prévoir : des politiques et des objectifs clairs; une organisation appropriée où il est défini sans équivoque à qui appartiennent les éléments d'actif et quelles sont les responsabilités qui s'y rattachent; du personnel compétent ainsi qu'une formation et une sensibilisation adéquates; des procédures et des normes appropriées; un contrôle de la performance et des procédures de vérification ou de réexamen propres à faire ressortir les lacunes et à les corriger; des procédures d'intervention en cas d'urgence régulièrement testées et réexaminées; des enquêtes sur les accidents. 
" L'industrie devrait partager en permanence son expérience quant à l'application des systèmes de gestion de la sécurité concernant les pipelines, et améliorer l'efficacité des différents éléments et techniques inhérents à ces systèmes, cela afin de réduire toujours davantage les risques et les accidents liés à des pipelines.

17.c.4 L'intégrité des pipelines devrait être préservée grâce à des mesures adéquates de maintenance, d'inspection et de contrôle ainsi qu'à une saine gestion.

" Parmi les moyens d'inspection et de contrôle figurent l'utilisation de racleurs intelligents, les tournées de vérification et la surveillance aérienne.

" Pour atteindre l'objectif consistant à améliorer sans cesse la performance en matière de sécurité, on peut, en plus de la maintenance ordinaire, procéder à des inspections et à un contrôle, échanger l'information à une échelle plus vaste parmi les exploitants, profiter des leçons tirées des incidents déclarés et tirer parti des nouvelles technologies ainsi que des autres avancées.

"Au fur et à mesure que les pipelines vieillissent, il peut être nécessaire d'exercer un contrôle de plus en plus étroit pour garantir leur intégrité. Il faudrait envisager de réexaminer et de revalider les pipelines et leurs conditions d'exploitation une fois qu'ils approchent de la fin de leur durée de vie nominale.

" Il devrait exister des politiques de remplacement des pipelines ou de segments de ceux-ci lorsqu'ils ne répondent plus aux normes de sécurité ou qu'ils arrivent au terme de leur durée de vie nominale.

17.c.5 Bien que les principes généraux qui s'appliquent à la planification en cas d'urgence intéressant les installations dangereuses valent également pour les pipelines, il peut être nécessaire de consentir à des efforts supplémentaires dans ce domaine, compte tenu de la situation particulière de chaque pipeline, par exemple des dangers associés aux substances transportées.

" La planification pour les situations d'urgence concernant les pipelines peut se compliquer du fait de certaines de leurs caractéristiques, notamment : le fait que les pipelines fonctionnent habituellement sans personnel; la longueur et l'emplacement des pipelines; la nécessité de pouvoir arrêter l'écoulement des substances ou de dépressuriser; la nécessité de ménager un accès au personnel d'intervention d'urgence. En outre, il faut tenir compte des aménagements voisins. Par exemple, là où les pipelines croisent une voie ferrée ou suivent un tracé parallèle à celle-ci, il est important de se coordonner avec les plans d'urgence mis en place par l'industrie ferroviaire.

" La planification en cas d'urgence devrait inclure une évaluation des risques relatifs au réseau de pipelines.

" Compte tenu de ces complications, il est essentiel de solliciter l'avis du personnel d'intervention d'urgence lors de la préparation, du réexamen et de la modification des plans d'urgence relatifs aux pipelines. 
- 17.c.6 Les entreprises responsables des pipelines devraient réexaminer et appliquer les procédures en vue de réduire l'ingérence de la part de tiers - puisque celle-ci est une source considérable d'accidents - et les étendre au besoin.

"Cela devrait se faire en collaboration avec les pouvoirs publics de toutes les régions et de tous les pays.

" Pour réduire l'ingérence de la part de tiers, on peut notamment veiller à ce que l'information appropriée au sujet de l'emplacement des pipelines dans une région donnée circule entre les parties concernées. De plus, il importe de faciliter les échanges entre l'exploitant du pipeline et les tiers, par exemple, selon un mode d'exclusivité par lequel l'information relative aux pipelines est fournie à une seule source reconnue, de grande diffusion.

- 17.c.7 Afin de faciliter l'apprentissage par l'expérience, les entreprises responsables des pipelines (de même que les pouvoirs publics et les autres parties prenantes) devraient améliorer le partage de l'information sur le perfectionnement de la sécurité des pipelines et la diffusion des dossiers sur les accidents ou quasi-accidents.

" Cela devrait comprendre l'information relative aux pipelines qui approchent de la fin de leur durée de vie théorique ou nominale. En ce qui concerne les pipelines désaffectés, on peut les démonter et les retirer, ou les laisser à l'abandon soit tels quels, soit en prenant certaines dispositions. Il faudrait soigneusement évaluer les risques associés à chacune de ces possibilités, au cas par cas, étant entendu que, parfois, la meilleure solution peut être une combinaison de ces différentes possibilités.

" Il faudrait mettre en commun l'information concernant l'ampleur des réseaux de pipelines, la quantité de substances qui y circulent de même que les analyses statistiques sur l'utilisation des pipelines à des fins de transport de substances dangereuses.

" L'information au sujet du lien entre les défaillances et les caractéristiques des pipelines devrait être rassemblée et partagée, et ce afin de mieux comprendre la nature et les causes des accidents (par exemple, l'âge, la taille, l'emplacement et la construction des pipelines). 


\section{NOTES}

1. Dans le cadre du présent document, les pays en développement et les pays à économie en transition qui reçoivent de l'aide dans le cadre d'activités de coopération technique sont appelés « pays bénéficiaires ».

2. Dans le cadre du présent document, les organismes d'aide comprennent les organismes d'aide bilatérale des pays fournissant une aide technique et financière aux pays en développement et aux pays à économie en transition, de même que les organisations internationales qui offrent une telle aide (par exemple, la Banque mondiale et les banques de développement régional).

3. Cela comprend notamment les sociétés d'ingénieurs, les cabinets d'avocats, les firmes d'experts-conseils et les groupes de conseillers financiers.

4. Dans le cadre du présent document, on entend par «interfaces des transports » : des zones fixes (désignées) où des substances dangereuses (marchandises dangereuses) sont transférées d'un moyen de transport à un autre (par exemple, transport routier à transport ferroviaire, ou navire à pipeline); transférées d'un véhicule à un autre, sans qu'il y ait changement de moyen de transport (par exemple, d'un camion à un autre); transférées d'un moyen de transport à une installation fixe ou d'une installation à un moyen de transport; entreposées de façon temporaire pendant le transfert entre moyens de transport ou entre équipements. Par conséquent, il y a aux interfaces des transports les activités et éléments suivants : manœuvres de chargement et de déchargement; installations de transfert; retenue ou entreposage temporaires des substances dangereuses pendant le transfert des cargaisons (par exemple, emmagasinage); prise en charge des véhicules endommagés et des déversements. Les gares de triage, les zones portuaires, les plates-formes de chargement et de déchargement des installations dangereuses, les gares routières et les gares multimodales où convergent le transport routier et le transport ferroviaire, les aéroports et les installations de transfert des installations fixes sont autant d'exemples d'interfaces des transports.

5. Cela comprend notamment les fabricants, les expéditeurs et les consignateurs, les transitaires, les groupeurs, les emballeurs, les courtiers et les négociants des marchandises.

6. Les ententes internationales actuelles en matière de transport des marchandises dangereuses devraient également s'appliquer à la manutention dans les interfaces des transports. La « réglementation» internationale constitue une bonne base pour harmoniser les règlements visant les différents moyens de transport.

7. Dans le cadre du présent document, on entend par «zones portuaires » les zones terrestres et maritimes délimitées comme telles par la loi, y compris les installations fixes et les bateaux (navires, embarcations diverses) sur place. Les substances dangereuses peuvent se trouver dans les zones portuaires afin d'y être chargées à bord de navires, de barges fluviales, de trains ou de camions ou d'en être déchargées, ou encore pour être expédiées ou reçues par pipeline; elles peuvent aussi faire partie d'une cargaison restant à bord d'un navire, sans manutention dans le port; enfin, elles peuvent aussi y être manutentionnées comme marchandises emballées à des fins de groupage ou de dissémination.

8. Un pipeline peut être défini comme un tube, habituellement cylindrique, où s'écoule une substance dangereuse d'un point à un autre. 
ANNEXES 


\section{ANNEXE I \\ EXPLICATION DES TERMES UTILISÉS}

Les explications des termes figurant ci-dessous sont destinées au présent document seulement; elles ne devraient pas être considérées comme des définitions généralement acceptées, et les termes ne devraient pas être pris comme des expressions harmonisées entre des pays et des organisations. On a utilisé dans la mesure du possible des définitions communes de ces termes.

acceptabilité-tolérabilité du risque (Acceptability/tolerability of risk)

Acceptation d'un risque pour profiter de certains avantages.

accident (ou accident chimique) (Accident, chemical accident)

Tout événement imprévu mettant en cause des substances dangereuses, qui cause ou peut causer des effets nocifs sur la santé, l'environnement ou la propriété. Cela exclut tout événement à long terme (comme la pollution chronique).

accident transfrontalier (Transboundary accident)

Accident mettant en cause des substances dangereuses, qui se produit dans une entité juridictionnelle, et qui entraîne ou peut entraîner des effets nocifs sur la santé ou l'environnement dans une autre entité juridictionnelle (à l'intérieur d'un pays ou dans un autre pays).

affiliés (Affiliates)

Entreprises dans lesquelles une autre entreprise est actionnaire minoritaire, sans exercer un véritable contrôle opérationnel.

aménagement du territoire (Land-use planning)

Comprend diverses procédures pour réaliser à la fois le zonage général et l'aménagement physique $\mathrm{du}$ territoire, ainsi que le processus décisionnel au cas par cas concernant l'implantation d'une installation ou d'autres aménagements.

analyse des dangers (Hazard analysis)

Détection des différents dangers d'un système donné, détermination des mécanismes par lesquels ces dangers pourraient entraîner des évènements indésirables et évaluation des conséquences de ces événements.

audit (Audit)

Examen systématique d'une installation dangereuse pour vérifier la conformité avec les règlements, les normes, les lignes directrices et (ou) les politiques internes. Cet examen comprend la production des rapports correspondants, mais pas les activités de suivi ultérieures. Un audit peut inclure l'examen effectué par la direction d'une installation dangereuse (auto-vérification ou audit interne) ou pour le compte de la direction, ou l'examen effectué par une tierce partie indépendante (audit externe).

autorités locales (Local authorities)

Administrations ou gouvernements locaux (p. ex. municipalités, comtés, provinces). Dans le cadre du présent document, il s'agit d'organismes responsables de la santé publique, des services de secours et d'incendie, de la police, de la sécurité professionnelle, de l'environnement, etc. 
autorités portuaires (Port authority)

Toute personne ou groupe de personnes habilités à gérer une zone portuaire.

capitaine (Master)

Toute personne, autre qu'un pilote ou un vigile, qui est responsable d'un navire.

cause(s) fondamentale(s) (Root cause)

La ou les principales causes qui conduisent à une action ou à une situation non sûres, se traduisant par un accident ou quasi-accident. Autrement dit, une cause fondamentale est une cause qui, si elle était éliminée, empêcherait le scénario d'évoluer vers un accident.

chargeur (Cargo interests)

Un expéditeur, transporteur, transitaire, groupeur, centre d'emballage, ou toute personne, compagnie ou institution engagé dans une activité, comme l'identification, le confinement, le conditionnement, l'emballage, l'arrimage, le marquage, l'étiquetage, la pose de plaques ou la documentation, selon le cas, pour des cargaisons où il y a présence de substances dangereuses, et gardant en tout temps le contrôle sur la cargaison.

communauté (ou collectivité) (Community)

Personnes vivant et (ou) travaillant près d'installations dangereuses, qui peuvent être touchées en cas d'accident chimique.

communication sur les risques (Risk communication)

Partage de l'information ou dialogue entre les intervenants au sujet des questions concernant la prévention, la préparation et l'intervention en matière d'accidents chimiques, et notamment les suivantes : risques pour la santé et l'environnement et leur portée; politiques et stratégies visant à gérer les risques et à prévenir les accidents; mesures à prendre pour atténuer les effets d'un accident. Dans le cadre du présent document, la communication sur les risques comprend le dialogue et le partage d'information entre la population, les pouvoirs publics, l'industrie et d'autres intervenants.

conduite

Voir « pipeline ».

conséquence (Consequence)

Résultat d'un événement donné.

contractants (Contractors)

Englobe tous les contractants et sous-traitants.

contrôle (ou surveillance) (Monitoring)

Recours à des vérifications, inspections, tournées, visites, échantillonnages, mesures, sondages, examens ou audits pour évaluer la conformité aux lois, règlements, normes, codes, règles ou pratiques qui sont en usage; comprend les activités déployées par les pouvoirs publics, l'industrie et des organismes indépendants.

\section{danger (Hazard)}

Propriété intrinsèque d'une substance, d'un agent, d'une source d'énergie ou d'une situation qui peut entraîner des conséquences indésirables.

direction (Management)

Toute(s) personne(s) ou entité légale (publique ou privée), notamment les propriétaires et les directeurs, qui ont la responsabilité de prendre des décisions pour l'entreprise. 
employé (Employee)

Toute personne travaillant à, ou pour, une installation dangereuse. Cela comprend aussi bien les membres de la direction que les travailleurs et les contractants.

entreprise (Enterprise)

Société ou corporation (y compris les corporations transnationales) dont les activités comprennent la production, le traitement, la manutention, l'entreposage, l'utilisation et (ou) l'élimination de substances dangereuses.

ergonomie (Ergonomics)

Discipline qui s'occupe d'adapter aux capacités humaines la conception des installations, des équipements, de l'exploitation et du milieu de travail.

évaluation des risques (Risk assessment)

Jugement de valeur sur la portée du risque, établi d'après une analyse des risques tenant compte de tout critère pertinent.

\section{fabricant (ou producteur) de produits chimiques (Chemical producer)}

Entreprise qui produit ou met au point des produits chimiques (notamment des produits de base et des produits spéciaux, des produits de consommation pour soins divers, des produits agrochimiques, pétrochimiques et pharmaceutiques).

\section{facteurs humains (Human factors)}

Les facteurs humains interviennent dans la conception des machines, des opérations et du milieu du travail pour qu'ils soient adaptés aux capacités, limitations et besoins humains (ils ont donc une portée plus grande que les questions liées à l'interface homme-machine). Ils sont basés sur l'étude des personnes présentes dans le milieu du travail (opérateurs, directeurs, personnel de maintenance, etc.) et sur les facteurs qui influent généralement sur les personnes dans leur relation avec l'installation technique (incluant l'individu, l'organisation et la technologie).

filiale (Subsidiary)

Entreprise dans laquelle une autre société est actionnaire majoritaire ou exerce un véritable contrôle opérationnel.

\section{gérance des produits (Product stewardship)}

Système de gestion des produits intéressant toutes les étapes de leur cycle de vie, y compris leur utilisation par le client et leur élimination (et dont l'objectif est d'améliorer constamment la sécurité des produits pour les personnes et l'environnement).

gestion des risques (Risk management)

Mesures prises pour garantir ou améliorer la sécurité d'une installation et de son fonctionnement.

incidents (Incidents)

Accidents et (ou) quasi-accidents.

\section{indicateurs}

indicateur d'activités (Activities indicator)

Moyen pour mesurer des actions ou des conditions qui, dans le contexte d'un programme concernant la prévention, la préparation et l'intervention en matière d'accidents chimiques, devraient garantir ou permettre les améliorations au niveau de la sécurité (p. ex. réduction du risque, améliorations de la gestion de la sécurité et de la culture de la sécurité, atténuation des effets nuisibles en cas d'accident). 
Ces indicateurs se présentent généralement sous la forme d'une liste de vérification non exhaustive d'exemples d'activités/conditions qui, pense-t-on, contribuent à l'amélioration de la sécurité. Les utilisateurs devraient utiliser les éléments de la liste qui conviennent à leur situation et ajouter d'autres éléments, selon les besoins.

indicateur de résultats (Outcome indicator)

Moyen pour mesurer les résultats, les effets ou les conséquences d'activités déployées dans le contexte d'un programme concernant la prévention, la préparation et l'intervention en matière d'accidents chimiques. Dans le cadre du présent document, les indicateurs de résultats sont conçus pour mesurer si les moyens pris permettent d'obtenir les résultats souhaités (c.-à-d. une opinion quantitative ou qualitative mesurable au sujet de la probabilité d'un accident, et (ou) de la portée des effets sur la santé humaine et l'environnement en cas d'accident).

indicateur de performance en matière de sécurité (Safety performance indicator)

Moyen pour mesurer les variations temporelles du niveau de sécurité (concernant la prévention, la préparation et l'intervention en matière d'accidents chimiques) à la suite des moyens engagés. Dans le cadre du présent document, les indicateurs de performance en matière de sécurité sont basés à la fois sur les indicateurs d'activités et les indicateurs de résultats.

\section{industrie chimique (Chemical industry)}

Ensemble des entreprises qui produisent, mettent au point et (ou) vendent des substances chimiques (notamment des produits chimiques de base et des produits spécialisés, des produits pour soins personnels, des produits agrochimiques, pétrochimiques et pharmaceutiques).

\section{information (Information)}

Faits, données ou autres connaissances qu'il est possible d'obtenir par un moyen quelconque, par exemple sous forme électronique, imprimée, sonore ou visuelle.

\section{inspection (Inspection)}

Contrôle effectué par les pouvoirs publics. Une ou plusieurs autres parties peuvent participer à l'inspection au nom des pouvoirs publics. Une inspection comprend le(s) rapport(s) qui en est (sont) issu(s), mais non le processus de suivi.

\section{installation dangereuse (Hazardous installation)}

Usine ou établissement industriel fixe où l'on produit, traite, manipule, entrepose, utilise ou élimine des substances dangereuses, sous une forme et en quantités telles qu'il existe un risque d'accident mettant en cause ces substances et pouvant porter gravement atteinte à la santé humaine, à l'environnement ou aux biens.

installations d'entreposage (Storage facilities)

Entrepôts, parcs de réservoirs et autres installations où l'on conserve des substances dangereuses.

interface des transports (Transport interface)

Zones fixes (déterminées) où des substances dangereuses (marchandises dangereuses) sont: transférées d'un mode de transport à un autre (p. ex. route à chemin de fer ou navire à pipeline); transférées à l'intérieur d'un mode de transport, d'une pièce d'équipement à une autre (p. ex. d'un camion à un autre); transférées d'un mode de transport à une installation fixe, ou d'une installation à un mode de transport; entreposées temporairement pendant le transfert entre des modes de transport ou des pièces d'équipement. Au niveau des interfaces des transports, se situent un certain nombre d'activités, et notamment les suivantes : chargement et déchargement, transfert à des installations, entreposage ou conservation temporaires de substances dangereuses pendant le transfert de fret (p. ex. emmagasinage), et manipulation de véhicules endommagés ou de marchandises déversées 
accidentellement. Parmi les exemples, on peut citer les suivants : gares de triage, zones portuaires, quais de réception/chargement à des installations dangereuses, terminaux pour le transport routier et le transport intermodal entre la route et le chemin de fer, les aéroports et les installations de transfert aux installations fixes.

\section{intervenant (ou partie prenante) (Stakeholder)}

Tout individu, groupe ou organisation qui est concerné, intéressé ou potentiellement affecté par la prévention, la préparation et l'intervention en matière d'accidents chimiques. Une description des divers groupes d'intervenants est présentée dans l'introduction de la présente publication sous « Portée ».

\section{navire (Ship)}

Toute construction flottante prévue pour aller sur la mer ou sur d'autres eaux - y compris celles qui sont utilisées sur les eaux intérieures - employée pour transporter les substances dangereuses.

organisme d'aide (Aid agency)

Les organismes d'aide comprennent: les organismes bilatéraux d'aide ou de développement, qui assistent techniquement et (ou) financièrement les pays en développement et ceux possédant une économie en transition; les organisations multilatérales fournissant une aide de ce type (p. ex. Banque Mondiale et banques pour le développement régional).

\section{partie prenante}

Voir « intervenant ».

\section{performance humaine (Human performance)}

Tous les aspects de l'action humaine correspondant à une exploitation sûre d'une installation dangereuse, à toutes les phases de celle-ci, depuis sa conception et sa construction, en passant par son exploitation et sa maintenance, jusqu'à sa mise hors service et fermeture.

pipeline (ou conduite) (Pipeline)

Tube, généralement cylindrique, utilisé pour faire passer une substance dangereuse d'un point à un autre. Dans le cadre du présent document, les pipelines comprennent toute installation auxiliaire, comme les stations de pompage et de compression.

plan de préparation aux situations d'urgence (ou plan d'urgence) (Emergency preparedness, emergency plan)

Plan écrit officiel qui expose, en fonction des accidents possibles mis en évidence et de leurs conséquences, comment faire face à de tels accidents et à leurs conséquences, aussi bien à l'intérieur qu'à l'extérieur de l'installation.

\section{poste d'amarrage (Berth)}

Tout dock, quai, môle, jetée, terminal portuaire ou structure similaire (flottant ou non), auquel un navire peut être amarré. Cela comprend aussi toute usine ou installation, autre qu'un navire, utilisée accessoirement ou occasionnellement pour le chargement ou le déchargement de substances dangereuses.

pouvoirs publics (Public authorities)

Organismes publics aux niveaux national, régional, local et international.

probabilité (Probability)

Possibilité qu'un événement donné se produise. 
quasi-accident (Near-miss)

Tout événement imprévu et soudain qui, sans l'effet atténuateur des dispositifs ou règles de sécurité, aurait pu exercer des effets nuisibles sur la santé, l'environnement ou les biens, ou provoquer une perte de confinement avec des conséquences néfastes mettant en cause des substances dangereuses.

raisonnablement possible (Reasonably practicable)

Tout ce qui est possible sous réserve que le coût des mesures ne soit pas exagérément disproportionné par rapport à la valeur des avantages qui en découlent.

rapport sur la sécurité (Safety report)

Présentation écrite des informations techniques et de celles touchant à la gestion et à l'exploitation qui se rapportent aux dangers d'une installation et à leur contrôle, à l'appui d'une justification de la sécurité de cette installation.

responsable de l'entrepôt (Warehouse keeper)

Personne responsable d'une installation d'entreposage, qu'elle soit sur le site d'une installation dangereuse ou à l'extérieur.

responsable de poste d'amarrage (Berth operator)

Toute personne ou groupe de personnes chargés (pendant une période donnée) de contrôler quotidiennement les opérations d'un poste d'amarrage.

risque (Risk)

Combinaison d'une conséquence et de sa probabilité.

substance dangereuse (Hazardous substance)

Élément, composé, mélange ou préparation qui présente un danger du fait de ses propriétés chimiques, physiques ou (éco)toxicologiques. Sont également considérées comme des substances dangereuses des substances non considérées normalement comme telles, mais qui, dans des cas spécifiques (p. ex. incendie, réaction d'emballement), réagissent avec d'autres substances ou dans des conditions opératoires particulières (température, pression) pour former des substances dangereuses.

\section{surveillance}

Voir « contrôle ».

système de gestion de la sécurité (Safety management system)

Partie du système général de gestion d'une entreprise qui comprend la structure organisationnelle, les responsabilités, les pratiques, les procédures, les méthodes et les ressources pour élaborer et mettre en oeuvre une politique de prévention des accidents chimiques. Le système de gestion de la sécurité porte généralement sur un certain nombre de questions, comprenant notamment les éléments suivants : organisation et personnel; identification et évaluation des dangers et des risques; contrôle opérationnel; gestion du changement; planification pour les urgences; suivi de la performance; audits et examens.

transfert de technologie (Technology transfer)

Passage d'un procédé ou d'une autre technologie intéressant la sécurité, d'un pays à un autre, qui comporte non seulement le transfert proprement dit, mais aussi l'application de cette technologie et l'exploitation de l'usine.

\section{travailleur (Worker)}

Toute personne travaillant à ou pour une installation dangereuse, mais ne faisant pas partie de la direction. Cela comprend les contractants. 


\section{triage (Triage)}

Évaluation de l'état clinique des individus exposés, avec désignation de priorités pour la décontamination, le traitement et le transport.

$\operatorname{vrac}($ Bulk)

Cargaison qui est transportée sans forme intermédiaire de confinement dans un espace de chargement.

zone portuaire (Port area)

Secteur terrestre et maritime délimité par voie législative. (Note : certaines zones portuaires peuvent se chevaucher. La législation devrait tenir compte de cette possibilité.) 


\section{ANNEXE II}

\section{ACRONYMES}

ACC : $\quad$ American Chemistry Council (anciennement CMA)

ACFPC : $\quad$ Association canadienne des fabricants de produits chimiques

ADN : $\quad$ Accord européen relatif au transport des marchandises dangereuses par voie de navigation intérieure (CEE-ONU)

ADR: $\quad$ Accord européen relatif au transport des marchandises dangereuses par route (CEE-ONU)

AEGL : $\quad$ Acute Exposure Guideline Levels

AGEE : $\quad$ Advisory Group on Environmental Emergencies (Groupe commun PNUE/BCAH de l'environnement)

AIEA : $\quad$ Agence internationale de l'énergie atomique

AIP : $\quad$ Association internationale des ports

AIPCN : $\quad$ Association internationale permanente des congrès de navigation

APELL : $\quad$ Information et préparation au niveau local (PNUE)

AQ : $\quad$ assurance de la qualité

BCAH : $\quad$ Bureau de la coordination des affaires humanitaires (ONU)

BEE : $\quad$ Bureau européen de l'environnement

BIAC : Comité consultatif économique et industriel auprès de l'OCDE

CAMEO : $\quad$ Computer-Aided Management of Emergency Operations

CAP : $\quad$ centre antipoison

CARAT : $\quad$ Chemical Accident Risk Assessment Thesaurus (OCDE)

CCPS : Center for Chemical Process Safety (American Institute of Chemical Engineers, AIChE)

CCR : $\quad$ Centre commun de recherche $(\mathrm{CE})$

CE : $\quad$ Commission européenne

CEE-ONU : Commission économique des Nations Unies pour l'Europe

CEFIC : $\quad$ Conseil européen des fédérations de l'industrie chimique

CETDG : $\quad$ Comité d'experts en matière de transport des marchandises dangereuses (ONU)

CIA : $\quad$ Chemical Industries Association (Royaume-Uni)

CISL : $\quad$ Confédération internationale des syndicats libres

CLC : $\quad$ Convention internationale sur la responsabilité civile pour les dommages dus à la pollution par les hydrocarbures

CSC-OCDE : Commission syndicale consultative auprès de l'OCDE

DTIE (PNUE) : Division de la technologie, de l'industrie et de l'économie du PNUE

EFCE : $\quad$ Fédération européenne du génie chimique

EPA : $\quad$ Environmental Protection Agency (États-Unis)

EPSC : $\quad$ European Process Safety Centre

EQR : $\quad$ évaluation quantitative des risques

FAO : Organisation des Nations Unies pour l'alimentation et l'agriculture

FISC : $\quad$ Forum intergouvernemental sur la sécurité chimique

FS : $\quad$ fiche signalétique

GRI : $\quad$ Global Reporting Initiative

GTAC : $\quad$ Groupe de travail sur les accidents chimiques

GTQ : $\quad$ gestion totale de la qualité

HSE : $\quad$ Health and Safety Executive (Royaume-Uni)

IBC : Recueil international de règles relatives à la construction et à l'équipement des navires transportant des produits chimiques dangereux en vrac

ICCA : Conseil international des associations chimiques 
ICEM : $\quad$ Fédération internationale des syndicats des travailleurs de la chimie, de l'énergie, des mines et des industries diverses

IChemE : $\quad$ Institute of Chemical Engineers (Royaume-Uni)

IGC : Recueil international de règles relatives à la construction et à l'équipement des navires transportant des gaz liquéfiés en vrac

IMDG : $\quad$ Code maritime international des marchandises dangereuses

IOCC : $\quad$ Comité de coordination interorganisations

IOMC : Programme interorganisations pour la gestion rationnelle des produits chimiques

ISGOTT : $\quad$ International Safety Guide for Oil Tankers and Terminals

MAHB : $\quad$ Major Accidents Hazards Bureau (CCR-CE)

MARPOL : Convention internationale pour la prévention de la pollution par les navires

MARS : $\quad$ Major Accident Reporting System (CE)

OACI : $\quad$ Organisation de l'aviation civile internationale

OCDE : Organisation de coopération et de développement économiques

OCDE/AEN : Agence de l'OCDE pour l'énergie nucléaire

OIG : $\quad$ organisation intergouvernementale

OIPC : $\quad$ Organisation internationale de protection civile

OIT : $\quad$ Organisation internationale du travail

OMI : $\quad$ Organisation maritime internationale

OMS : $\quad$ Organisation mondiale de la santé

ONG : $\quad$ organisation non gouvernementale

ONU : $\quad$ Organisation des Nations Unies

ONUDI : $\quad$ Organisation des Nations Unies pour le développement industriel

OPRC : $\quad$ Convention internationale sur la préparation, la lutte et la coopération en matière de pollution par les hydrocarbures

OSHA : $\quad$ Occupational Safety and Health Administration (États-Unis)

PET :

PHSE : $\quad$ Programme sur l'hygiène et la sécurité de l'environnement

PIRG : $\quad$ Public Interest Research Groups (États-Unis)

PISSC : $\quad$ Programme international sur la sécurité des substances chimiques

PME : $\quad$ petites et moyennes entreprises

PNUE : $\quad$ Programme des Nations Unies pour l'environnement

REMPEC: Centre régional méditerranéen pour l'intervention d'urgence contre la pollution marine accidentelle (PNUE/OMI)

RID : $\quad$ Règlement concernant le transport international ferroviaire des marchandises dangereuses (CEE-ONU)

RTDG : $\quad$ Recommandations relatives au transport des marchandises dangereuses (ONU)

SGH : $\quad$ Système général harmonisé de classification et d'étiquetage des produits chimiques

SOLAS : Convention internationale pour la sauvegarde de la vie humaine en mer

SSE : $\quad$ Sécurité, santé et environnement

UE : $\quad$ Union européenne

UNITAR : Institut des Nations Unies pour la formation et la recherche

USEPA : $\quad$ United States Environmental Protection Agency

WWW : $\quad$ World-wide Web 


\section{ANNEXE III \\ INDEX DE MOTS CLÉS}

Le présent index de mots clés a été conçu pour aider le lecteur à localiser les paragraphes qui comprennent une ou des références à un sujet ou à un intervenant particuliers. Il ne s'agit pas d'un index de sujets; les paragraphes mentionnés contiennent simplement le mot clé.

Les références croisées présentées dans cette annexe renvoient à des concepts apparentés (bien que non nécessairement identiques). En utilisant cet index, il faut garder à l'esprit que des termes légèrement différents sont parfois employés dans les différentes parties des Principes directeurs, même si c'est le même sujet ou un sujet étroitement apparenté qui est traité.

Les références renvoient aux paragraphes des parties $A$-E. L'index ne renferme pas de références aux sections d'introduction des Principes directeurs, ni aux autres annexes. Il ne renferme pas non plus de références au texte explicatif des Parties $A-E$ des Principes directeurs (c.-à-d. le texte en italique au début de certaines sections). Enfin, l'index de mots clés ne renvoie pas non plus aux Règles d'or, si ce n'est de les mentionner dans les entrées concernant les principaux intervenants considérés.

\section{acceptabilité/tolérabilité du risque}

1.18; 3b.2; encadré texte sur la recherche (chapitre 4); 5d.8; 7.11; $16 \mathrm{~b} .12$

affiliés, entreprises affiliées (ou activités affiliées)

2i. $11-2 \mathrm{i} .18,16 \mathrm{c} .18 ; 16 \mathrm{c} .19$

aide mutuelle ou assistance mutuelle (voir également transfrontalière [coopération])

1.19, encadré texte sur les PME (chapitre 1); 5a.14; 5b.10; 16a.8; 16c.41

aménagement du territoire (planification et ou politiques) (voir également implantation)

2c.1 - 3; 3a.10; 3b1 - 4; 4e.3; encadré texte sur la recherche (chapitre 4); 5.2 (note); 6.1; 6.2; 6.3; 6.4; $6.5 ; 6.6 ; 13.3$ (note); 16a.2; 16b.8; 16c.2; 16c.20; 16c.34; 16c.35; 17a.1; 17c.2

application (de la loi)

1.12; 1.14; 3a.7; 3a.21; 3b.1; 3c.2; 3c.9; 3c.13; 6.3; 9.2; 15c.1; 17a.6; 17a.13; 17b.1

association commerciale voir association industrielle

association industrielle ou association commerciale

1.17; 1.19; encadré texte sur les PME (chapitre 1); 2c.5; 2i.4; 2i.5; 2i.6; encadré texte sur la Gestion responsable (chapitre 2); 3a.4; 14c.4; 15a.10; 15a.12; 16b.9; 16c.9; 16c.24

assurance

2a.14; 2g.9; $15 \mathrm{~d} .2$

audit (voir également inspection)

1.19; 2a.15; 2c.7; 2d.21; 2g.1 - 14; 2i.16; encadré texte sur la GRI (chapitre 2); 3a.21; 3c.1; 3c.4; 3c.8; 3c. $13 ; 17 \mathrm{~b} .10 ; 17 \mathrm{c} .3$

autorités locales (ou fonctionnaires) voir pouvoirs publics autorités provinciales voir pouvoirs publics 
autorités régionales voir pouvoirs publics

cadre de contrôle

1.12; 3a.2; 3a.4; 3а.6; 3a.7; 3a.16; 15c.1; 16c.2; 16c.34; 17a.13

changement voir « changement, gestion du »

changement, gestion du

2a.15; 2b.8; 2c.7; 2d.2; 2d.38; 2f.1; 2f.2; 3a.16; 5a.12; 5c.8; 5c.18; 6.4; 15b.3; encadré texte " enquêtes sur les incidents » (chapitre 15); 16c.29; 16c.36

comité de la sécurité

$2 \mathrm{~d} .26 ; 2 \mathrm{~d} .27,5 \mathrm{~d} .5$

communauté, collectivité (voir également public)

$1.2 ; 1.7 ; 1.15 ; 1.18 ; 1.19 ; 2$ c. $3 ; 2$ c. $13 ; 2$ g.5; 2i.8; 2i.16; 3a.4; 3a.21; 3b.3; section 4a; encadré texte sur la représentation d'une communauté (chapitre 4); 5a.9; 5a.12; 5a.14; 5a.19; 5b.2; 5b.9; 5c.2; 5c.8; 5c.11; 5c.17; 5c.18; 5c.21; 5d.1-5; 5d.8; encadrés texte sur APELL (chapitres 5 et 16); 7.1; 7.2; 7.5; 7.6; 7.10 12; 7.17; 10.7; 10.10; 10.12; 10.16; 10.19; 11c.1; 15d.1; 16a.6; 16b.2; 16b.5; 16b.18; 16c.16; 16c.17; 16c.26; 16c.41 (voir également les « Règles d'or »)

communication (générale)

1.4; 2a.1; 2a.2; 2a.9; 2a.17; 2b.4; 2d.17; 2d.23; 2d25; 2d.26; 2d.36; 2d.39; 2e.1; 3b.4; 3c.12; 3c.13; 4a.3; 4b.1; 5a.4; 5a.8; 5a.10; 5a.11; 5a.14; 5a.16; 5a.18; 5a.19; 5b.2; 5b.5; 5c.5; 5c.11; 5c.20; 5d.3; 5d.6; encadré texte sur l'intervention d'urgence pour les installations médicales (chapitre 5); encadré texte sur APELL (chapitres 5 et 16); 7.6; 7.7; 7.8; 7.10; 7.13; 7.17; 8.4; 11.1; 15a.11; 16a.3; 16a.4; 16c.5; 17a.15; 17b.1; 17c.6

communication avec le public voir information du public

communication des risques voir information du public/communications sur les risques; voir également communication

conception technique voir conception/ingénierie/construction

conception/ingénierie/construction ( $\mathrm{d}$ 'une installation dangereuse)

2a.1; 2a.15; 2b.8; 2c.2; 2c.4 - 2c.21; 2d.2; 2d.5; 2d.17; 2d.21; 2d.42; 2d.43; 2e.1; 2f.1; 2g.11; 2i.10; 3a.16; 3c.1; encadré texte " enquêtes sur les incidents » (chapitre 15); 16c.1 - 4; 16c.9; 16c.11; 16c.12; 16c.16; 17a.1; 17a.7; 17c.1; 17c.3; 17c.7

conduites voir pipelines

conséquence(s)

2a.16; 2b.7; 2c.2; 2i.14; 3a.1; 3a.2; 3a.12; 3a.13; 5a.3; 5b.1; 5c.23; 6.4; 10.1; 10.11; 11c.1; 12.1; 12.2; 13.2; 14b.2; 15a.1; 16b.5, 17a.12, 17c.1

consignes (d'exploitation) voir procédures

construction voir conception/ingénierie/construction 


\section{contractants}

1.2; 1.7; 1.9; 2a.18; 2c.18; 2c.21; 2d.1; 2.d2; 2d.15; 2d.16; 2d.20; 2d.25; 2d.26; 2d.33; 2d.34; 2d.40;

2d.43; 2e.1; 2f.5; 2h.1; 2i.1; 2i.5; 2i.10; 5b.2 (note); 14c.3; 16c.17; 17a.4; 17a.7; 17a.8

contrôle voir inspections; voir audits

culture de la sécurité (de l'entreprise)

2a.1 - 3; 2a.6; 2a.7; 2a.14; 2c.7; 2d.23; 14c.3

culture de la sécurité de l'entreprise

2a.1-6

dangers (analyse, évaluation, identification ou étude) (voir également évaluation du risque)

2a.15; 2a.16; 2a.17; 2b.1; 2b.4; 2b.7; 2c.6; 2c.17; 2c.18; 2d.16; 2d.20; 2d.34; 2d.43; 2e.1; 2f.1; 2f.2; 2g.2; 2g.10; 2g.11; 2h.1; 2i.1; 2i.8; 2i.11; 3a.12; 3b.2; 3c.8; 3c.10; 4a.3; 5a.3; 5b.1; 5b.8; 6.1; 7.1; 10.10; 14c.3; 16c.3; 16c.8; 16c.9; 16c.27; 17a.5; 17a.15; 17a.20; 17c.2; 17c.5

déclaration (ou notification d'incidents)

1.9; 2a.17; 2d.31; 2g.2; 3a.19; 5a.17; 8.1; 11a.3; 12.2; 14b.1; 14b.2; 14c.1; 14c.2; 14c.3; 14c.5; 15b.1; $16 a .5 ; 17 a .16 ; 17 b .4 ; 17 c .4$

démolition (d'une installation)

2a.1; 2h. $1 ; 3$ c. 1

directeur d'usine voir direction

direction/directeurs (d'une installation dangereuse) (voir également industrie)

1.2; 1.5 - 1.9; 1.13; 1.14; encadré texte sur les PME (chapitre 1); 2a.1; 2a.2; 2a.4; 2a.5; 2a.6; 2a.8, 2a.9, 2a.10; 2a.12; 2a.14; 2a.16; 2b.1; 2b.2; 2b.7; 2b.8; 2c.1; 2c.2; 2c.3; 2c.12; 2c.13; 2c.17; 2c.18; 2c.21; 2d.1; 2d.2; 2d.5; 2d.7; 2d.9; 2d.10; 2d.11; 2d.13; 2d.15; 2d.17 - 20; 2d.22 - 26; 2d.28 - 31; 2d.33 - 36; 2d.38; 2d.39; 2d.40; 2d.43; 2d.45; 2e.1; 2e.2; 2f.1; 2f.2; 2f.3; 2f.5; 2g.2; 2g.8; 2g.9; 2g.10; 2h.1; 2i.7; 2i.18; 3a.4; 3a.12; 3a.14; 3a.19; 3b.2; 3c.5; 3c.13; 4b.1; 4b.2; 5a.1; 5b.1; 5b.3; 5b.6; 5b.8; 5b.9; 5c.2; 5c.5; 5c.16; 5d.5; 5d.6; 5d.7; 9.1; 9.2; 10.2; 12.1; 14a.1; 14b.2; 14c.1; 14c.2; 14c.3; 15a.1; 15a.2; 15a.12; 15b.1 - 4; 15c.3; encadré texte "enquêtes sur les incidents » (chapitre 15); 16b.5; 16c.1; 16c.5; 16c.17; 16c.23 - 26; 16c.28; 16c.29; 16c.32; 16c.37; 16c.39; 17a.5; 17a.6; 17a.8; 17a.9; 17a.13; 17a.15; 17a.16; 17a.20 (voir également les « Règles d'or »)

documentation (ou documents)

2a.7; 2a.17; 2c.11; 2c.17; 2c.18; 2f.1; 2f.2; 2g.3; 2i.10; 3c.1; 3c.4; 3c.9; 4e.3; 5c.13; 10.19; 12.2; 14b.2; 15a.4; 15a.5; 15b.1; 15c.1; encadré texte " enquêtes sur les incidents » (chapitre 15); 16b.2; 16b.8; 16c.9; 17a.3; 17a.10; encadré texte sur les interfaces des transports (chapitre 17)

éducation voir formation

effet domino

2c.13; 2c.14; 3b.3; 5a.4; 5b.10; 16c. $9 ; 17 b .1$

employés (voir également travailleurs; voir également personnel)

1.2; 1.9; 1.11; 1.13; 2a.1 - 5; 2a.8; 2a.9; 2a.10; 2a.12; 2a.14; 2b.5; 2c.6; 2c.7; 2.c.9; 2c.16; 2c.18; 2d.1; 2d.2; 2d.5; 2d.6; 2d.10; 2d.14; 2d.17; 2d.18; 2d.20 - 26; 2d.28 - 35; 2d.38; 2d.39; 2d.41; 2d.43 - 45; 2f.4; 2g.4; 2g.10; 2i.7; 2i.9; 2i.12; 2i.16; 3a.4; 3c.3; 3c.12; 3c.13; 4b.1; 5a.19; 5b.1-3; 5b.9; 5c.2; 5c.11; 
5d.5; 7.12; 9.2; 10.2; 14c.3; 15a.3; 15b.1; 15b.3; 16c.5; 16c.9; 16c.17; 16c.24; 16c.25; 16c.28; 16c.29; 16 c. $32 ; 16$ c. $39 ; 17$ a. $4 ; 17$ a. 7

\section{enquête(s)}

2a.2; 2a.15; 2d.31; 2d.36; 2g.2; 2i.18; 10.19; 14b.2; 15a.1 - 14; 15 b.1 - 4; 15c.1 - 5; 15d.1; 15d.2; encadré texte " enquêtes sur les incidents » (chapitre 15); 17c.3

entreposage (de substances dangereuses) (voir également entrepôt)

1.3; 2a.7; 2a.15; 2c.2; 2c.4; 2c.14; 2c.17; 2d.6; 2d.8; 2d.20; 2i.1; 2i.15; 5b.1; 5b.8; 16c.9; 17a (note); $17 a .11 ; 17 a .12$

entrepôt (voir également entreposage)

2d.6; encadré texte sur APELL (chapitre 16); 17a (note)

entreprise(s) (voir également industrie; voir également direction; voir également producteurs)

$1.3 ; 1.4 ; 1.13 ; 1.15 ; 1.19$; encadré texte sur les PME (chapitre 1); 2a.1; 2a.2; 2a.6; 2a.7; 2a.9; 2a.10; 2 a.12 - 14; 2a.17; 2b.9; 2c.1; 2c.2; 2c.5; 2c.6; 2c.11; 2c.13; 2c.20; 2c.21; 2d.1; 2d.2; 2d.15; 2d.16; 2d.23; 2d.24; 2d.26; 2d.44; 2d.45; 2g.2; 2g.4; 2g.8 - 10; 2g.14; 2i.2 - 7; 2i.11 - 18; 3a.3; 3a.5; 3a.8; 3a.21; 3b.2; 3c.1; 3c.10; 3c.13; 4b.1; 5a.14; 5b.1; 5b.2; 5b.5; 5b.10; 5c.3; 5c.6; 7.4; 9.3; 10.2; 10.19; 14a.1; 14b.2; 14c.2; 14c.3; 15a.12; 15b.3; 15b.4; 15d.2; encadré texte " enquêtes sur les incidents » (chapitre 15); 16b.9; 16b.14; 16c.1; 16c.2; 16c.4; 16c.12; 16c.15 - 21; 16c.26; 16c.27; 16c.28; 16c.30; 16c.34

essais voir exercices/essais

États voir pouvoirs publics

évaluation du risque

1.18; 2a.17; $2 \mathrm{~b} 1$ - 9; 2c.2; 2d.21; 2d.43; 2i.8; 2i.12; 3a.13; 3b.2; encadré texte sur la recherche (chapitre 4 ); 5a.3; 5c.8; 10.9; 15c.3; 17a.20; 17c.1; 17c.2; 17c.5

exercices/essais (aux fins de la planification pour les situations d'urgence)

2a.15; 2d.37; 5a.12; 5a.18; 5c. $2 ; 5$ c. $8 ; 5$ d. $4 ; 5$ d. $5 ; 17$ c. 3

\section{exposition aiguë}

2b.3; encadré texte sur la recherche (chapitre 4); 5a.2 (note); 5c.11; 10.4; 10.9; 12.2; 13.3; 13.3 (note)

\section{facteurs humains ou erreur humaine}

1.17; 2b.3; 2b.6; 2c.4; 2c.6; 2c.9; 2d.42 - 46; 5a.12; encadré texte « enquêtes sur les incidents » (chapitre 15); 17a.7

fermer (une installation) voir mettre hors service

fermer (voir aussi mettre hors service)

2d.43; 2e.1; 2e.2; 2h.1; 3c.9; 5b.1; 5b.2; 17a.7; 17c.1

fonctionnaires voir pouvoirs publics

\section{formation, sensibilisation, éducation}

1.6; 1.19; 2a.4; 2a.10; 2a.15; 2b.9; 2c.7; 2c.9; 2c.10; 2c.11; 2c.17; 2d.2; 2d.5; 2d.6; 2d.17; 2d.21; 2d.23; 2d.24; 2d.26; 2d.28; 2d.34 - 43; 3a.18; 3a.20; 3a.21; 3c.4; 3c.8; 3c.11; 3c.12; 4a.3; 4b.1; 5a.11; 5a.12; 5a.19; 5b.9; 5c.8; 5c.18; 5c.20; 5d.6; 5d.8; 6.5; 7.5; 7.8; 7.9; 7.12; 7.14; 10.8; 10.16; 12.1; 14c.3; 15a.4; 15a.11; 15a.12; 15b.3; encadré texte « enquêtes sur les incidents » (chapitre 15); 16b.2; 16b.4; 16b.5; 
16b.6; 16b.8; 16b.12; 16c.1; 16c.9; 16c.11; 16c.12; 16c.16; 16c.21; 16c.24; 16c.29; 16c.31; 16c.39; 16c.40; 16c.41; 17a.4; 17a.7; 17c.3

fournisseur (de substance dangereuse ou d'installation dangereuse)

1.19; encadré texte sur les PME (chapitre 1); 2c.20; 2d.6; 2i.2; 2i.5; 2i.6; 2i.7; 2i.8; 5b.1; 16c.3; 16 c.7 $14 ; 16 c .24 ; 16 c .25 ; 16 c .33 ; 16 c .36$

gérance des produits

$1.10 ; 2$ i. $1 ; 2$ i. $5 ; 16$ c. 24

gestion des risques

2a.13; 2a.16 (note); 2b.5; 3a.12 (note); 15 c.3

implantation (voir aussi aménagement du territoire)

2c.1-3; 3a.14; 3b.1; 3c.1; 16a.6; 16b.8; 16c.14; 16c.20; 17a.1

industrie (voir également entreprise, direction, producteurs)

1.2; 1.3; 1.6; 1.13; 1.17; 1.19; 2a.17; 2c.5; 2g.1; 2g.4; 2g.6; 2g.14; 2i.3 - 6; 3a.4; 3a.5; 3a.6; 3a.9; 3a.12; 3a.16; 3а.20; 3a.21; 3c.1; 3c.3; 3c.12; 3c.13; 3c.14; 4a.1; 4a.2; 5a.7; 5a.8; 5a.9; 5a.12; 5a.20; 5b.1; 5b.5; 5b.9; 5c.17; 5d.3; 7.11; 7.13; 8.4; 10.9; 14a.1; 14b.2; 14b.3; 14c.5; 15a.1; 15a.10; 15a.12; 16b.2; 16b.8; 16b.9; 16b.18; 16c.5; 16c.24; 16c.26; 16c.31; 16c.32; 16c.34; 16c.39; 16c.41; encadré texte sur les organisations intergouvernementales (chapitre 16); 17a.15; 17a.18; 17c3; 17c.5; 17c.6; 17c.7 (voir également les « Règles d'or »

information (du public) ou communications sur les risques

1.12; 1.13; 2g.4; 3c.3; 4a.1; 4a.2; 4a.3; 4e.2; 5b.9; 5c.5; 5c.20; 5d.3; 5d.8; encadré texte sur APELL (chapitre 5$) ; 7.5 ; 7.6 ; 7.9 ; 7.11 ; 16 c .5$

inspection ou inspecter (installations) (voir également audit)

1.12; 1.14; 2c.14; 2c.17; 2c.18; 2d.17; 2d.44; 2e.1; 2e.2; 2f.1; 2g.1; 2g.3; 2g.6; 2g.7; 3a.12; 3c.1; 3c.3 - 13; 10.18; 15c.1; encadré texte « enquêtes sur les incidents » (chapitre 15); 17a.1; 17a.13; 17b.10; 17c.4

interface(s) des transports

17a (note); 17a.1; 17a.2; 17a.5 - 9; 17a.11 - 16; 17a.18; 17a.19; encadré texte sur les interfaces des transports (chapitre 17)

limites d'exposition aiguë (ou directives sur les limites d'exposition aiguë)

encadré texte sur la recherche (chapitre 4); 5 a.2 (note); 13.3 (note)

main-d'œuvre (voir également employés; voir également travailleurs, personnel)

maintenance

2a.1; 2a.5; 2a.15; 2c.7; 2c.17; 2c.18; 2d.2; 2d.8; 2d.9; 2d.21; 2d.43; 2d.46; 2e.1; 2e.2; 2g.11; 2i.13; 3c.1; 15a.3; 15a.4; encadré texte « enquêtes sur les incidents » (chapitre 15); 16b.6; 16c.3; 16c.9; 16c.12; 16c.21; 17a.7; 17a.10; 17b.5; 17c.2; 17c.3; 17c.4

mettre hors service (une installation)

2a.1; 2a.15; 2d.43; 2h. $1 ; 3$ c. $1 ; 17$ c.3

ministre voir pouvoirs publics 
mise en service (d'une installation)

2a.15; 2c.17; 2c.18; 2c.19; 2d.11; 2d.46; 2e.1; 2f.3; 3a.14; 16c.11; 16c.12; 16c.13

modification

2c. $2 ; 2$ c. $4 ; 2$ c.5; 2c. $15 ; 2$ c. $18 ; 2$ d. $2 ; 2$ d.46; 2f.1; 2f.3 - 5; 3a.14; 3b. $2 ; 16$ c. $12 ; 16$ c. $29 ; 16$ c.39

notification d'incidents voir déclaration (d'incidents)

ONG (organisation non gouvernementale)

1.6; encadré texte sur les PME (chapitre 1); 3b.2; 4e.1 - 4; encadré texte sur la représentation d'une communauté (chapitre 4); 5d.8; 11c. $1 ; 15 \mathrm{~d} .1$

organisation(s) syndicale(s) (voir également syndicat)

$1.19 ; 4$ b. $1-4$ b. $3 ; 5$ d. $5 ; 5 d .6 ; 5 d .7$

organisations internationales (ou intergouvernementales)

2d.19; 4b.3; 4d; 5d.5; 5d.7; 16b.3; 16b.8; 16b.15; 16c.6; 16c.24; 16c.34; encadré texte sur les organisations intergouvernementales (chapitre 16); $17 \mathrm{~b} .3$

organismes d'aide (ou donneurs)

16b. 2 - 10; 16b.16; 16b.17; 16c. 25

paramédical voir personnel médical

participation du public (ou de la communauté) (participation du public au processus décisionnel) 3a.4; 3a.14; 4a.1; encadré texte représentation d'une communauté (chapitre 4); 5a.5; 5c.2; 5d.1; 5d.4; 6.7; 7.11; 7.15; 16a.6; 16b.2

personnel (voir également employés, travailleurs)

1.13; 2a.1; 2a.4; 2a.14; 2a.15; 2a.17; 2b.5; 2c.7; 2c.10; 2c.13; 2c.14; 2c.16; 2c.18; 2d.3; 2d.11; 2d.22; 2d.23; 2d.40; 2d.45; 2f.4; 2g.3; 2g.5; 3c.4; 3c.11; 5a.9; 5a.12; 5a.15; 5a.16; 5a.17; 5b.2; 5c.5 - 8; 5c.11; 5c.13; 5c.15; 5c.17; encadré texte sur la préparation pour les urgences dans le cas des installations médicales (chapitre 5); 7.14; 8.1; 9.2; 10.2; 10.4; 10.5; 10.6; 10.8 - 11; 10.13; 10.15- 19; 11c.1; 12.1; 14b.2; 15a.12; 16a.6; 16c.24; 16c.39; 16c.41; 17a.3; 17a.14; 17a.16; 17c.2

personnel d'intervention ou autorités responsables en cas d'urgence ou intervenants en cas d'urgence (voir également " pouvoirs publics », personnel médical/sanitaire)

2a.17; 2b.5; 2d.3; 2d.6; 2g.5; 5a.5; 5a.8; 5a.9; 5a.12; 5a.15; 5a.17; 5a.19; 5b.2; 5b.3; 5b.8; 5c.5 - 11; 5c.15; 5c.23; 7.14; 8.1; 8.2; 9.2; 10.1; 10.2, 10.4 - 11; 10.16 - 19; 11c.1; 14b.2; 15a.4; 16c.41; 17a.3; 17a.14; 17a.16; 17c. $2 ; 17$ c.5

personnel médical, sanitaire, paramédical 5a.8; 5a.9; 5c.7; 5c.8; 5c.18; encadré texte sur l'intervention d'urgence pour les installations médicales (chapitre 5) 10.2; 10.4; 10.8; 10.13; 10.15; 13.4

pipelines (conduites)

5b.8; 17a (note); 17a.7; 17a.19; 17b (note) 17c (note), 17c.1 - 7

pire des scénarios

5a.2; 5a.11; 5a.13; 5b.1 
plan(ification) ou préparation pour les situations d'urgence

- en général : 2a.15; 2c.11; 3a.10; 3a.13; 3a.15; 5a.1 - 7; 5a.9 - 16; 5a.18; 5a.19; 5a.20; 5b.1; 5b.2; 5b.3; bb.8; 5b.10; 5c1 - 8; 5c.10 -16; 5c.18; 5c.20; 5c.21; 5c.23; 5d.1; 5d.2; 5d.4 - 7; encadré texte sur ce type de planification pour les installations médicales (chapitre 5); encadré texte sur APELL (chapitre 5 ); $7.4 ; 7.13 ; 7.15 ; 8.1 ; 8.2 ; 9.1 ; 9.2 ; 9.3 ; 10.1 ; 10.3 ; 10.10 ; 10.12 ; 10.14 ; 10.16 ; 10.17 ; 10.19$; 11a.2; 11c.1; 15c.3; 16a.1; 16a.3; 16a.4; 16b.1; 16c.26; 16c.34; 16c.35; encadré texte sur APELL (chapitre 16); 17a.14; 17b.1;17b.11; 17b.13; 17c.5

- extérieur de l'installation : 2a.17; 5a.1; 5a.5; 5a.6; 5a.10; 5a.12; 5b.1; 5b.2; 5b.3; 5b.8; 5b.10; 5c.1; 5c.2; 5d.1; 5d.2; 9.1; 17a.14

- à l'installation : 2a.17; 5a.1; 5a.5; 5a.6; 5a.10; 5a.12; 5b.1; 5b.2; 5b.8; 5c.1; 5c.2; 5c.3; 5c.5; 5c.13; 5d.1; 5d.2; 5d.4; 7.4; 7.15; 9.2; 16a.1; 16a.3; 16a.4; 16c.26; 17a.14

planification voir plan(ification) pour les situations d'urgence

PME (petites et moyennes entreprises)

1.13; 1.15; 1.19; encadré texte sur les PME (chapitre 1); 2g.9; 2i.2; 2i.6; 3a.6; 3a.13; 3a.15; 3a.21; 5b.1; $5 c .3$

police voir pouvoirs publics

politique de sécurité

2a.1; 2a.7 - 15; 2a.17; 2d.1; 16c.17; 16c.18

pompiers voir pouvoirs publics

population voir public

pouvoirs publics (voir également personnel d'intervention, personnel médical/sanitaire)

- en général : $1.2 ; 1.6 ; 1.8 ; 1.12 ; 1.13 ; 1.14 ; 1.17 ; 1.19$; encadré texte sur les PME (chapitre 1); 2a.7; 2a.15; 2a.18; 2b.7; 2c.1; 2c.2; 2c.3; 2c.5; 2c.17; 2d.31; 2d.44; 2e.2; 2g.4; 2g.8; 2i.5; 2i.6; 2i.9; 2i.12; 3a.1 - 15; 3a.17 - 21; 3b.1 - 3b.4; 3c.1; 3c.2; 3c.3; 3c.5; 3c.6; 3c.8 - 14; 4a.1 - 3; 5a.1; 5a.7; 5a.9; 5a.12; 5a.14; 5a.19; 5a.20; 5b.1; 5b.2; 5b.8; 5b.9; 5c.1 - 5; 5c.9; 5c.10; 5c.13; 5c.15; 5c.16; 5c.17; 5c.20; 5c.22; 5d.3; encadré texte sur la planification pour les situations d'urgence dans les établissements médicaux (chapitre 5 ); $6.1 ; 6.2 ; 6.4 ; 7.2 ; 7.9 ; 7.11 ; 7.13 ; 8.4 ; 9.2 ; 9.3 ; 10.9 ; 10.18 ; 10.21 ; 11$ a.2; 13.1; 14a.1; 14b.1; 14b.2; 14b.3; 14c.1; 15a.1; 15a.12; 15c.1 - 4; 16a.1; 16a.2; 16a.4; 16a.5; 16a.7; 16a.8; 16a.9; 16b.2; 16b.4; 16b.16; 16b.17; 16b.18; 16c.1; 16c.3; 16c.5; 16c.9; 16c.10; 16c.14; 16c,17; 16c.26; 16c.32 - 41; 17a.2; 17a.9; 17a.13; 17a.15; 17a,16; 17a.17; 17a.19; 17a.20; 17b.1; 17b.11; 17c.6; 17c.7; encadré texte sur les interfaces des transports (chapitre 17); (voir également les « Règles d'or »)

- pompiers/service d'incendie : 2d.3; 2d.6; 5a.5; 5a.8; 5b.3; 5c.5; 5c.8; 5c.9; 10.9

- autorités locales : 1.2; 1.15; encadré texte sur les PME (chapitre 1); 2c.2; 2d.6; 2i.8; 3a.2; 3c.12; 5a.14; 5a.19; 5b.6; 5c. $2 ; 6.2 ; 9.2 ; 10.9 ; 16$ c. $5 ; 16$ c. $12 ; 16$ c. $26 ; 16$ c. 35

- police : $5 a .5 ; 5$ a. $8 ; 5 c .5 ; 5 c .8 ; 10.9$

- autorités régionales : $1.2 ; 3$ c.12; 5c.2; 6.2; 10.7; 10.10; 10.18

préparation pour les urgences voir plan(ification) 
préparation voir plan(ification) pour les situations d'urgence

\section{procédures}

1.7; 2a.1 - 4; 2a.13; 2a.14; 2a.15; 2a.17; 2c.4; 2c.6; 2c.7; 2c.8; 2c.11; 2c.14; 2c.17; 2d.2 - 6; 2d.9; 2d.20; 2d.24; 2d.34; 2d.36; 2d.38; 2d.42; 2e.1; 2f.1; 2f.2; 2f.3; 2f.5; 2g.2; 2g.8; 2g.10;2h.1; 2i.7; 2i.10; 3c.3; 3c.12; encadré texte sur la recherche (chapitre 4 ); 5a.1; 5a.17; 5b.3; 5c.3; 5c.8; 5c.12; 5c.13; 5c.15; 5c.22; encadré texte sur la planification pour les urgences dans les installations médicales (chapitre 5); encadré texte sur APELL (chapitre 5); 6.4; 7.15; 10.21; 13.1; 14b.2; 14c.1; 14c.3; 15a.2; 15b.1; 15b.3; encadré texte " enquêtes sur les incidents » (chapitre 15); 16a.4; 16a.6; 16a.8; 16b.5; 16b.12; 16b.16; 16c.1; 16c.12; 16c.25; 16c.29; 16c.33; 16c.40; 17a.6; 17a.8; 17a.11; 17b.2; 17b.5; 17b.12; 17c.3

producteurs (substances dangereuses)

$1.3 ; 1.10 ; 2 \mathrm{i} .1$

public (non les pouvoirs publics) (voir également communauté)

$1.2 ; 1.12 ; 1.13 ; 2 a .6 ; 2 a .11 ; 2 b .5 ; 2$ c.2; $2 \mathrm{~g} .4 ; 2 \mathrm{~g} .8$; encadré texte sur la gestion responsable (chapitre 2); 3a.4; 3a.12; 3a.14; 3a.20; 3c.1; 3c.3; 3c.13; 4a.1; 4a.3; 4e.2; 4e.4; 5a.2; 5a.5; 5a.7; 5a.12; 5a.18; 5a.19; 5b.1; 5b.5; 5b.9; 5c.5; 5c.8; 5c.20; 5c.23; 5d.3; 5d.8; 6.7; 7.1; 7.2; 7.3; 7.4; 7.5; 7.6; 7.8; 7.9; 7.11; 7.13; 7.14; 7.15; 7.17; 8.1; 8.4; 10.1; 10.2; 10.3; 10.8; 10.9; 11a.1; 11a.2; 11a.3; 11b.1; 11b.2; 14b.2; 15a.12; 15c.1; 15c.2; encadré texte "enquêtes sur les incidents » (chapitre 15); 16a.4; 16c.5; 16c.14; 16c.20; 16c.26; 16c.31; 16c.34; encadré texte sur APELL (chapitre 16); 17a.15; $17 \mathrm{~b} .11$ (voir également les « Règles d'or »)

\section{quasi-accident}

1.4 (note); $1.9 ; 2$ a.2; 2a.3; 2d.44; 3a.19; 5a.12; 14a.1; 14b.2; 14b.3; 14c.2; 14c.3; 15a.5; 15a.12; 15b.1; 15b.2; 15b.3; 16c. $13 ; 17$ a. $16 ; 17$ c. 7

\section{rapport de (sur la) sécurité}

2a.16 - 18; 2c.17; 2g.2; 3a.12; 3a.15; 3b.2; 3c.1; encadré texte sur le GRI (chapitre 2); 5b.1; $17 a .5$

recherches (voir également université)

1.19; 2c.4; 2d.9; 2i.14; 3a.20; 4c; encadré texte sur la recherche (chapitre 4); 5a.15; 13.4; 16b.11, $16 c .31$

\section{réglementation}

encadré texte sur les PME (chapitre 1); 2a.7; 2b.9 (note); 2c.21; 2d.15; 2i.5; 3a.2; 3a.3; 3a.5; 3a.6; 3a.16; 3a.21; 3c.1; 3c.12; 3c.13; 14b.3; 15c.1; 15c.3; 16c.9; 16c.14; 17a.12; 17a.18 (note); 17a.19; 17b.1; $17 \mathrm{~b} .8$

réparations (voir également maintenance) 2d.46; 2e. $1 ; 2$ f. $1 ; 2$ f. $3 ; 17 b .5$

représentant des employés ou représentant des travailleurs ou représentant en sécurité (voir également syndicats)

1.2; 2a.3; 2a.10; 2a.12; 2a.14; 2c.16; 2d.6; 2d.10; 2d.26; 2d.28; 2d.30; 2d.34; 2d.38; 2d.44; 2g.2; 2g.13; 2i.9; 2i.12; 4b.1; 5a.19; 5d.5; 5d.6; 14c.3; 15a.3; 15 b.3

représentant des travailleurs (voir représentant des employés, syndicat)

représentant en sécurité (voir représentant des employés) 
scénarios d'accidents (voir également pire des scénarios)

2b.6; 2c.6; 5a.2; 5a.11; 5a.13; 5a.15; 5b.1; 5b.8; 5c.3; 6.4; encadré texte « enquêtes sur les incidents » (chapitre 15)

sécurité intrinsèque

2 c. $4 ; 2$ c. $6-8 ; 3$ a. 9

sensibilisation voir formation

sous-traitants voir contractants

surveillance voir contrôle

syndicat(s) (voir également organisation syndicale, représentant des employés)

1.2 (note); 1.19; encadré texte sur les PME (chapitre 1); 2d.19; 3a.4; 4b.1; 16b.8

\section{système de gestion de la sécurité}

1.17; 2a.7; 2a.13 - 15; 2a.17; 2c.7; 2d.15; 2g.1; 2g.8; 3a.17; 3c.1; 15a.6; 16c.24; encadré texte sur les organisations intergouvernementales (chapitre 16); 17a.6; 17c.3

systèmes d'alarme

5a.18; $7.2 ; 8.1 ; 11 a .1 ; 12.1$

tolérabilité voir acceptabilité

transfert de technologie

2d.9; 2i.7 - 10; 16b.14; 16c.1 - 4; 16c.6; 16c.7; 16c.9; 16c.10; 16c.12; 16c.13; 16c.14; 16c.27; 16c.33; 16c.34; 16c.36; 16c.38; encadré texte sur les organisations gouvernementales (chapitre 16)

transfrontalier(s), transfrontalières(s)

- coopération (voir également aide mutuelle) 5a.14; 5a.20; 5c.21; 7.16; 16a.14; 16b.1; 16c.41

- effets d'un accident 3a.11 (note); 5c.21; 16a.1; 16a.2; 16a.3; 16a.4; 16a.5; 16a.6

transport (dans le contexte des substances dangereuses)

1.3; 2a.15; 2b.8; 2c.1; 2c.4; 2c.6; 2c.15; 2d.33; 2i.1, encadré texte sur la recherche (chapitre 4); 5a.1; 5a.2; 5a.4; 5a.9; 5a.12; 5b.8; 17a.1 - 21; 17b.8; 17c.1; 17c.6; 17c.7; encadré texte sur les interfaces des transports (chapitre 17)

travailleurs (voir également employé(e), personnel)

1.19; encadré texte sur les PME (chapitre 1); 2a.10; 2a.12; 2b.8; 2d.1; 2d.6; 2 d.17 - 19; 2d.25; 2d.26; 2d.28; 2d.34; 2d.35; 2d.38; 2d.43; 2f.2; 2g.2; 2g.10; 2g.13; 4b.1; 4b.2; 5d.5; 5d.6; 5d.7; 15b.3; 16b.2; 16b.18; 16c.2 (voir également les « Règles d'or »); 2d.39; 5c.2; 10.2; 17a.7; encadré texte sur les interfaces des transports (chapitre 17)

université ou établissement universitaire (voir également recherches)

3b.2; section 4c; encadré texte sur la recherche (chapitre 4); 5c.10; 15d.1; 16c.31

zone portuaire

5b.10; 17b.1 - 6; 17b.11 - 14 


\section{ANNEXE IV \\ SÉLECTION DE RÉFÉRENCES}

On trouve dans la présente annexe une liste de sites Internet et de documents pouvant intéresser les lecteurs des Principes directeurs; on peut aussi y consulter un répertoire des instruments juridiques pertinents, dont certains sont cités dans ces Principes. En outre, l'annexe comprend la liste complète des rapports issus d'ateliers et de sessions spéciales de l'OCDE qui ont été utilisés pour préparer les Principes.

Ces listes n'ont PAS la prétention d'être exhaustives; elles ont plutôt été établies à partir de suggestions du Groupe de travail sur les accidents chimiques et du Groupe de rédaction de l'OCDE. L'objectif consistait à signaler des documents pertinents (en particulier ceux qui fournissaient des informations complémentaires sur la mise en ceuvre des Principes directeurs) et facilement accessibles au public. La plupart des documents énumérés sont disponibles en anglais, en français ou dans ces deux langues.

\section{SITES INTERNET}

\section{Pays membres de l'OCDE}

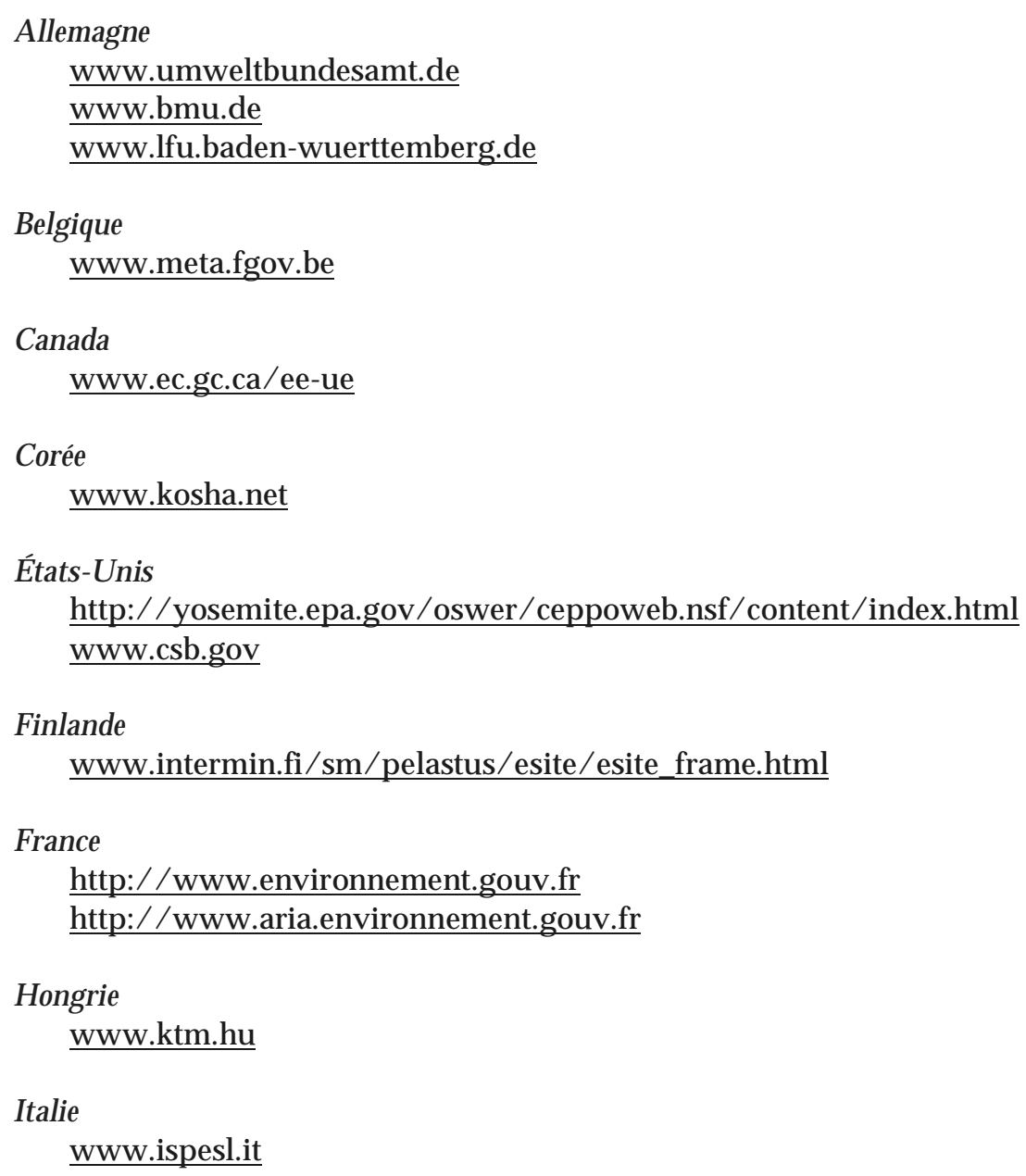


Mexique

www.semarnat.gob.mx

Norvège

www.dbe.no

Pays-Bas

www.vrom.nl

République tchèque

www.env.cz

www.vubp.cz

www.bozpinfo.cz

Royaume-Uni

www.hse.gov.uk (Health and Safety Executive)

www.environment-agency.gov.uk (UK Environment Agency)

www.sepa.org.uk (Scottish Environment Protection Agency)

Slovaquie

www.enviro.gov.sk

Suède

www.srv.se

www.av.se

www.environ.se

Suisse

www.umwelt-schweiz.ch

\section{Organisations internationales}

Bureau de la coordination des affaires humanitaires www.reliefweb.int/ochaunep/ (Groupe commun PNUE/BCHA de l'environnement)

Commission économique des Nations Unies pour l'Europe www.unece.org/env/teia/welcome.html

Commission européenne

http://europa.eu.int/comm/environment/civil

http://mahbsrv.jrc.it/

Organisation de coopération et de développement économiques www.oecd.org/env/accidents

Organisation internationale du travail

http://www.ilo.org/public/french/protection/safework/index.htm

Organisation maritime internationale

www.imo.org/home.asp 
Organisation mondiale de la santé www.who.int

Programme des Nations Unies pour l'environnement www.uneptie.org/pc/apell (APELL) www.unep.org/DEPI/disastermanagement.asp (Mise en œuvre des politiques environnementales) www.reliefweb.int/ochaunep/ (Groupe commun PNUE/BCHA de l'environnement)

Programme international sur la sécurité des substances chimiques www.who.int/pcs

\section{Organisations industrielles}

American Chemistry Council www.americanchemistry.com

Center for Chemical Process Safety (American Institute of Chemical Engineers) www.aiche.org/ccps/

Conseil européen des fédérations de l'industrie chimique (CEFIC) www.cefic.org

Conseil international des associations chimiques www.icca-chem.org

European Process Safety Centre http://www.epsc.org/

Kemikontoret (association des industries chimiques suédoises) www.chemind.se/Kemikontoret/main.htm

\section{Syndicats}

Fédération internationale des syndicats de travailleurs de la chimie, de l'énergie et des industries diverses www.icem.org

\section{Autres organisations non gouvernementales}

Bureau européen de l'environnement www.eeb.org

Clary-Meuser Research Network http:/ / www.mapcruzin.com/ http://www.mapcruzin.com/rmp_maps/rmp_resources.htm

Greenpeace http://www.greenpeaceusa.org 
Public Interest Research Groups (États-Unis)

http://www.pirg.org

Right to Know Network

http://www.rtk.net/

\section{Établissements universitaires}

The Wharton School of the University of Pennsylvania (États-Unis)

http://grace.wharton.upenn.edu/risk/ (Risk Management and Decision Processes Center)

Université de Bordeaux 1 (France)

http://portaildurisque.iut.u-bordeaux1.fr (Institut universitaire de technologie)

\section{PUBLICATIONS}

\section{Généralités}

Agence européenne pour l'environnement (1999). L'environnement dans l'Union européenne à l'aube du XXIe siècle (chapitre 3.8), Copenhague.

Centre commun de recherche de la CE (1997). Guidance on the Preparation of a Safety Report to Meet the Requirements of Council Directive 96/82/EC (Seveso II), Luxembourg, ISBN 92-828-1451-3.

Centre commun de recherche de la CE (1998). Guidelines on a Major Accident Prevention Policy and Safety Management System, as required by Council Directive 96/82/EC (Seveso II), Luxembourg, ISBN 92828-4664-4.

Centre commun de recherche de la CE (1998). Lessons Learnt from Accidents: Proceedings of a Seminar held on 16-17 October 1997, Luxembourg, ISBN 92-828-2845-x.

CISL (1986). Is There a Bhopal Near You? Unions Drive to Prevent Chemical Disasters Worldwide. Trade Union Principles for the Prevention of Chemical Disasters, Bruxelles.

CISL-ICEF (1985). Trade Union Report on Bhopal, disponible à l'adresse www.Bhopal.net.

Journal of Hazardous Materials (1999). The SEVESO II Directive (96/82/EC) on the control of major accident hazards involving dangerous substances (divers articles connexes), Vol. 65, $\mathrm{n}^{\circ}$ 1-2, Elsevier Science.

Journal of Loss Prevention in the Process Industries (1999). International Trends in Major Accidents and Activities by the European Commission towards Accident Prevention (divers articles connexes), Vol. 12, No 1, Elsevier Science.

OIT (1988). La maîtrise des risques d'accident majeur : guide pratique, Genève.

OIT (1991). Prevention of Major Accident Hazards (an ILO Code of Practice), Genève. 
PNUE (1988). APELL Handbook: Awareness and Preparedness for Emergencies at Local Level: A Process for Responding to Technological Accidents, ISBN 92-807-1183-0.

PNUE (1990). APELL Storage of Hazardous Materials: A Technical Guide for Safe Warehousing of Hazardous Materials, ISBN 92-807-1238-1.

PNUE (1994). Annotated APELL Bibliography, ISBN 92-807-1411-2.

PNUE (1995). APELL Worldwide, ISBN 92-807-1527-5.

PNUE (2001). APELL for Mining: Guidance for the Mining Industry in Raising Awareness and Preparedness for Emergencies at Local Level, ISBN 92-807-2035.

Royaume-Uni (1999). A Guide to the Control of Major Accident Hazards Regulations (implementing Seveso II in Great Britain), ISBN 0-7176-1604-5 (L111).

USEPA (1998). Risk Management Program: RMP*Submit RMP*Info, disponible à l'adresse www.epa.gov/ceppo/factsheets/rmpfact3.pdf.

USEPA (1999). Chemicals in Your Community, disponible à l'adresse www.epa.gov/ceppo/pubs/chem-in-comm.pdf.

USEPA (1999). Guidance for Auditing Risk Management Plans (RMPs), disponible à l'adresse www.epa.gov/ceppo/pubs/audit_gd.pdf.

\section{Prévention}

Centre commun de recherche de la CE (1999). Guidance on Inspections as required by Article 18 of the Council Directive 96/82/EC (Seveso II), Luxembourg, ISBN 92-828-5898-7.

EPSC (1996). Safety Performance Measurement, edited by Jacques van Steen, ISBN 0852953828.

OCDE (2003). Guidance for Safety Performance Indicators (publication prévue en 2003).

USEPA (1996). Chemical Accident Prevention and the Clean Air Act Amendments of 1990, disponible à l'adresse www.epa.gov/ceppo/factsheets/caa-gnrl.pdf.

USEPA (1996). Risk Management Planning: Accidental Release Prevention; Final Rule: Clean Air Act section 112(r), disponible à l'adresse www.epa.gov/ceppo/factsheets/rmprule.pdf.

USEPA (1996). Risk Management Program: Accidental Release Prevention, disponible à l'adresse www.epa.gov/ceppo/factsheets/rmp-fs.pdf.

\section{Petites et moyennes entreprises}

OCDE (2001). Chemical Hazards and their Control in Small and Medium-Sized Enterprises (SMEs) - an Approach to Hazard Reduction, Paris (brochure). 


\section{Évaluation des dangers et des risques}

AIChE, Center for Chemical Process Safety (1999). Guidelines for Consequence Analysis of Chemical Releases, New York, ISBN 0-8169-0786-2.

AIChE, Center for Chemical Process Safety (2002). Guidelines for Chemical Process Quantitative Risk Analysis, (2 ${ }^{\text {nd }}$ Edition), New York, ISBN 0-8169-0720-X.

AIEA (1996). Manual for the Classification and Prioritization of Risks Due to Major Accidents in Process and Related Industries, ISSN 1011-4289.

AIEA (1998). Guidelines for Integrated Risk Assessment and Management in Large Industrial Areas, ISSN 1011-4289.

Arendt, J. S. et D. K. Lorenzo (2000). Evaluating Process Safety in the Chemical Industry: A User's Guide to Quantitative Risk Analysis, AIChE, New York (aperçu à l'intention des gestionnaires).

Greenberg, Harris R. et Joseph J. Cramer. (1991). Risk Assessment and Risk Management for the Chemical Process Industry, Van Nostrand Reinhold, New York, ISBN 0-442-23438-4.

OCDE (1989). Évaluation de la gestion des risques d'accidents liés aux activités industrielles, Paris, monographie de l'OCDE sur l'environnement no 19 .

PIRG (1999). At Risk and In the Dark: Will Companies In Our Communities Reduce Their Chemical Disaster Zones?, États-Unis, disponible à l'adresse www.pirg.org/reports/enviro/in_the_dark/index.html.

Pitblado, Robin et Robin Turney (1996). Risk Assessment in the Process Industries (2nd Edition), Institution of Chemical Engineers, Rugby, Warwickshire, Royaume-Uni, ISBN 0852953232.

PNUE (1992). APELL Technical Guide on Hazard Identification and Evaluation in a Local Community, ISBN 92-807-1331-0.

USEPA (1999). Risk Management Program Guidance for Offsite Consequence Analysis, disponible à l'adresse www.epa.gov/ceppo/ap-ocgu.htm\#112r.

\section{Aspects sanitaires}

OCDE (1996). Orientations concernant les aspects des accidents chimiques touchant à la santé, Paris.

OMS (1997). Assessing the Health Consequences of Major Chemical Incidents: Epidemiological Approaches, WHO Regional Publications, European Series No. 79, ISBN 9289013435.

PISSC (1999). Public Health Role and Chemical Incidents: Guidance for National and Regional Policy Makers in the Public/Environmental Health Roles.

PISSC, OCDE, PNUE-IE et OMS (publication conjointe) (1994). Aspects des accidents chimiques touchant à la santé, orientations visant la sensibilisation aux accidents chimiques, la préparation aux situations d'urgence et l'intervention, à l'intention des responsables de la santé et de l'intervention d'urgence, monographie de l'OCDE sur l'environnement n 81, Rapport technique du PNUE-IE nº19, Paris [à traduire]. 


\section{Préparation aux situations d'urgence}

PNUE/BCAH (Groupe commun PNUE/BCAH de l'environnement). Directives pour l'élaboration d'un Plan national d'intervention écologique, disponible à l'adresse www.reliefweb.int/ochaunep/tools/directives.htm [à traduire].

Royaume-Uni (1999). Emergency Planning for Major Accidents - Control of Major Accident Hazards Regulations (implementing Seveso II in Great Britain), ISBN 0-7176-1695-9 (HSG191).

UNEP/OCHA (Groupe commun PNUE/BCAH de l'environnement). Establishing a National Environmental Emergency Response Mechanism, disponible à l'adresse www.reliefweb.int/ochaunep/tools/prep.htm.

USEPA (1987). Technical Guidance for Hazardous Analysis, Emergency Planning for Extremely Hazardous Substances, disponible à l'adresse www.epa.gov/ceppo/pubs/tech.pdf.

USEPA (1990). NRT-2 - Developing a Hazardous Materials Exercise Program - A Handbook for State and Local Officials, disponible à l'adresse http://ntl.bts.gov/DOCS/254.html.

USEPA (1996). The National Response Team's Integrated Contingency Plan Guidance (One Plan), disponible à l'adresse http://www.epa.gov/docs/fedrgstr/EPA-GENERAL/1996/June/Day-05/pr23388.pdf.

USEPA (1999). RMPs and Local Emergency Planning Committees (LEPCs), June, disponible à l'adresse www.epa.gov/ceppo/pubs/lepcrmp.pdf.

USEPA (2000). The Emergency Planning and Community Right-to-Know Act, disponible à l'adresse www.epa.gov/ceppo/factsheets/epcra.pdf.

USEPA (2001). LEPCs and Deliberate Releases: Addressing Terrorist Activities in the Local Emergency Plan, disponible à l'adresse www.epa.gov/ceppo/factsheets/lepcct.pdf.

USEPA (2001). List of Lists - Consolidated List of Chemicals (by CAS \#) Subject to the Emergency Planning and Community Right-to-Know Act (EPCRA) and Section 112(r) of the Clean Air Act, disponible à l'adresse www.epa.gov/ceppo/pubs/title3.pdf.

USEPA (2001). NRT-1 - Hazardous Materials Planning Guide, disponible à l'adresse http://Itdomino1.icfconsulting.com/nrt/home.nsf/resources/RRTPages1/\$File/cleanNRT10_12_di stiller_complete.pdf.

\section{Aménagement du territoire}

Centre commun de recherche de la CE (1999). Guidance on Land-Use Planning, as required by Council Directive 96/82/EC (Seveso II), Luxembourg, ISBN 92-828-5899-5. 


\section{Sensibilisation et information du public}

Centre commun de recherche de la CE (1994). General Guidelines for the Content of Information to the Public - Directive 82/501/EEC - Annex VII, Luxembourg, ISBN 92-826-9053-9.

États-Unis, Chemical Safety Network. How to Increase Public Awareness and Improve Emergency Notification: Beach Cities CAER (Community Awareness and Emergency Response), disponible à l'adresse http://yosemite.epa.gov/oswer/ceppoweb.nsf/content/result.htm?OpenDocument\&CAER.

États-Unis, City of Deer Park, Texas Local Emergency Planning Committee. Shelter in Place Information for Children, disponible à l'adresse www.wally.org.

USEPA (2000). The Emergency Planning and Community Right-to-Know Act, disponible à l'adresse www.epa.gov/ceppo/factsheets/epcra.pdf.

\section{Intervention d'urgence et suivi}

OCDE, DTIE (PNUE) et Groupe commun PNUE/BCAH de l'environnement (publication conjointe) (2002). International Directory for Emergency Response Centres, disponible à l'adresse http://webnet1.oecd.org/EN/document/0 „EN-document-520-14-no-21-22715-0,00.html.

PNUE/BCAH (Groupe commun PNUE/BCAH de l'environnement). Directives pour l'évaluation écologique après l'alerte chimique, disponible à l'adresse

www.reliefweb.int/ochaunep/tools/guidchim.htm [à traduire].

\section{Transport lié aux installations fixes}

Nations Unies. Recommandations relatives au transport des marchandises dangereuses.

OCDE et IMO (publication conjointe) (1996). Directives relatives à la sécurité des substances chimiques dans les zones portuaires, Paris [à traduire].

OMI (1995). Code maritime international des marchandises dangereuses (Code IMDG).

PNUE (2000). TransAPELL: Guidance for Dangerous Goods Transport Emergency Planning in a Local Community, ISBN 92-807-1907-6.

PNUE et OMI (publication conjointe) (1996). APELL for Port Areas: Preparedness and Response to Chemical Accidents in Ports.

\section{ATELIERS et SESSIONS SPÉCIALES DE L'OCDE concernant les ACCIDENTS CHIMIQUES}

(Les rapports issus de ces ateliers et sessions spéciales, présentés en ordre chronologique, ont été utilisés pour préparer les présents Principes directeurs.)

Atelier sur la prévention des accidents liés aux substances dangereuses, Bonnes pratiques de gestion (22-25 mai 1989, Berlin), rapport publié en 1990 dans la série des monographies de l'OCDE sur l'environnement ( $\left.\mathrm{n}^{\circ} 28\right)$. 
Atelier sur la communication d'informations au public et le rôle des travailleurs dans la prévention des accidents et l'intervention (11-14 septembre 1989, Stockholm), rapport publié en 1990 dans la série des monographies de l'OCDE sur l'environnement (no 28).

Atelier sur le rôle des pouvoirs publics dans la prévention des accidents graves et dans l'aménagement du territoire en fonction des risques d'accidents graves (19-22 février 1990, Londres), rapport publié en 1990 dans la série des monographies de l'OCDE sur l'environnement (no 30).

Atelier sur la préparation et l'intervention en cas d'urgence ainsi que sur la recherche dans les domaines de la prévention des accidents, de la préparation et de l'intervention en cas d'urgence (7-10 mai 1990, Boston), rapport publié en 1990 dans la série des monographies de l'OCDE sur l'environnement (no 31).

Workshop on Prevention of Accidents Involving Hazardous Substances - The Role of the Human Factor in Plant Operations (22-26 avril 1991, Tokyo), rapport publié en 1991 dans la série des monographies de l'OCDE sur l'environnement ( $\left.\mathrm{n}^{\circ} 44\right)$.

Workshop on Strategies for Transporting Dangerous Goods by Road: Safety and Environmental Protection (2-4 juin 1992, Karlstad, Suède), rapport publié en 1993 dans la série des monographies de l'OCDE sur l'environnement (no 66).

Workshop on Chemical Safety in Port Areas (18-23 octobre 1993, Naantali, Finlande), rapport publié en 2001 dans la série des monographies de l'OCDE sur l'environnement (no 93).

Special Session on Chemical Accident Prevention Preparedness and Response at Transport Interfaces (30 novembre-1er décembre 1993, Paris), rapport publié en 1995 dans la série des monographies de l'OCDE sur l'environnement ( $\left.n^{\circ} 94\right)$.

Workshop on Small and Medium-Sized Enterprises in relation to Chemical Accident Prevention, Preparedness and Response (3-6 mai 1994, Toronto, Canada), rapport publié en 1995 dans la série des monographies de l'OCDE sur l'environnement (no 95).

Workshop to Promote Assistance for the Implementation of Chemical Accident Programmes ("International Assistance Activities related to Chemical Accident Prevention, Preparedness and Response") (6-7 février 1995, Divonne, France), rapport publié en 1997 dans la série du PHSE sur les accidents chimiques (no 3) (atelier conjoint de l'OCDE et de la CEE-ONU).

Workshop on Risk Assessment and Risk Communication in the Context of Chemical Accident Prevention, Preparedness and Response (11-14 juillet 1995, Paris), rapport publié en 1997 dans la série du PHSE sur les accidents chimiques ( $\left.\mathrm{n}^{\circ} 1\right)$.

Workshop on Pipelines (Prevention of, Preparedness for, and Response to Releases of Hazardous Substances) (3-6 juin 1996, Oslo), rapport publié en 1997 dans la série du PHSE sur les accidents chimiques $\left(\mathrm{n}^{\circ} 2\right)$.

Workshop on Human Performance in Chemical Process Safety: Operating Safety in the Context of Chemical Accident Prevention, Preparedness and Response (24-27 juin 1997, Munich), rapport publié dans la série du PHSE sur les accidents chimiques : no 4.

Workshop on New Developments in Chemical Emergency Preparedness and Response (3-6 novembre 1998, Lappeenranta, Finlande), rapport publié dans la série du PHSE sur les accidents chimiques (no 5). 
CCPS/OECD Conference and Workshop on Chemical Accident Investigations, (2-6 octobre 2000, Orlando, Florida), rapport publié dans le site Internet de l'OCDE en 2002.

Workshop on Integrated Management of Safety, Health, Environment and Quality, (26-29 juin 2001, Séoul), rapport publié en 2002 dans la série du PHSE sur les accidents chimiques (no 9).

Workshop on Audits and Inspections related to Chemical Accident Prevention, Preparedness and Response (6-9 mars 2001, Madrid), rapport publié en 2002 dans la série du PHSE sur les accidents chimiques $\left(\mathrm{n}^{\circ} 8\right)$.

Special Session on Environmental Consequences of Chemical Accidents (28 novembre 2000, Paris), rapport publié en 2002 dans la série du PHSE sur les accidents chimiques (nº 7).

\section{INSTRUMENTS JURIDIQUES}

\section{CONVENTIONS}

\section{Accidents chimiques (généralités)}

Organisation internationale du travail

Convention concernant la sécurité dans l'utilisation des produits chimiques au travail Adoptée le 25 juin 1990. Disponible à l'adresse www.ilo.org/ilolex/cgi-lex/convdf.pl?C170.

Convention concernant la prévention des accidents industriels majeurs Adoptée le 22 juin 1993. Disponible à l'adresse www.ilo.org/ilolex/cgi-lex/convdf.pl?C174.

Commission économique des Nations Unies pour l'Europe

Convention sur les effets transfrontières des accidents industriels Adoptée le 17 mars 1992. Disponible à l'adresse www.unece.org/env/teia/welcome/html.

Convention de 1992 sur la protection et l'utilisation des cours d'eau transfrontières et des lacs internationaux

Adoptée le 17 mars 1992. Disponible aux adresses www.unece.org/env/water et www.iwac-riza.org.

\section{Information du public}

\section{Commission économique des Nations Unies pour l'Europe}

Convention sur l'accès à l'information, la participation du public au processus décisionnel et l'accès à la justice en matière d'environnement. Adoptée le 25 juin 1998. Disponible à l'adresse www.unece.org/env/pp/treatytext.htm. 


\section{Transport de marchandises dangereuses}

Commission économique des Nations Unies pour l'Europe (ADR)

Accord européen relatif au transport international des marchandises dangereuses par route Adopté le 30 septembre 1957 (modifiée en 1985) [à traduire]. Disponible à l'adresse www.unece.org/trans/danger/publi/adr/adr_f.html.

Accord européen relatif au transport international des marchandises dangereuses par voie de navigation intérieure (ADN)

Adopté le 25 mai 2000. Disponible à l'adresse

www.unece.org/trans/main/dgdb/adnconf/adnfdoc/f-accord.pdf.

Règlement concernant le transport international ferroviaire des marchandises dangereuses (RID), annexe 1 à l'appendice B de la Convention relative aux transports internationaux ferroviaires (COTIF)

\section{Organisation maritime internationale}

Convention internationale de 1973 pour la prévention de la pollution par les navires, telle que modifiée par le Protocole de 1978 relatif (MARPOL 73/78)

Adoptée le 2 novembre 1973.

Convention internationale sur la préparation, la lutte et la coopération en matière de pollution par les hydrocarbures (OPRC 1990)

\section{ACTES DU CONSEIL DE L'OCDE}

The OECD Recommendation of the Council concerning Chemical Accident Prevention, Preparedness and Response (C(92)1/Final) (en cours de révision).

Décision du Conseil sur l'échange d'informations concernant les accidents susceptibles de provoquer des dommages transfrontières $(\mathrm{C}(88) 84$ (Final)).

Décision-recommandation du Conseil concernant la communication d'informations au public et la participation du public au processus de prise de décisions visant les mesures de prévention et d'intervention applicables aux accidents liés aux substances dangereuses (C(88)85(Final)).

Recommandation du Conseil sur l'application du principe pollueur-payeur aux cas de pollution accidentelle (C(89)88(Final)).

\section{DIRECTIVE DE LA CE}

Directive 96/82/CE du Conseil, du 9 décembre 1996, concernant la maîtrise des dangers liés aux accidents majeurs impliquant des substances dangereuses. (Directive Seveso II), disponible aux adresses http://europa.eu.int/scadplus/leg/fr/lvb/121215.htm et http://www.mahbsrv.jrc.it.

(Note : Une proposition de modification de cette directive est à l'étude.) 


\section{ANNEXE V}

\section{INFORMATION DOCUMENTAIRE}

Les présents Principes directeurs ont été élaborés dans le cadre du Programme de l'OCDE sur les accidents chimiques, sous la supervision du groupe d'experts responsable de la gestion de ce programme, le Groupe de travail sur les accidents chimiques (GTAC).

Le présent document a été produit dans le cadre du Programme interorganisations pour la gestion rationnelle des produits chimiques (IOMC).

\section{L'OCDE}

L'Organisation de coopération et de développement économiques (OCDE) est une organisation intergouvernementale au sein de laquelle des représentants de 30 pays industrialisés (d'Europe, d'Amérique du Nord et du Pacifique) ainsi que de la Commission européenne se réunissent afin de coordonner et d'harmoniser leurs politiques, d'examiner des questions d'intérêt commun et de coopérer à la résolution de problèmes internationaux. La majeure partie des travaux de l'OCDE sont menés à bien par plus de 200 comités spécialisés et groupes subsidiaires composés de délégués des pays membres. Des observateurs issus de différents pays possédant un statut spécial auprès de $\mathrm{l}^{\prime} \mathrm{OCDE}$, d'organisations internationales et d'organisations non gouvernementales (y compris des représentants de l'industrie et des travailleurs) assistent à un grand nombre des ateliers et réunions diverses de l'OCDE. Le Secrétariat de l'OCDE, qui a son siège à Paris (France), assiste les comités et les groupes subsidiaires; il se compose de directions et de divisions.

\section{Le Programme sur les accidents chimiques}

Le travail de l'OCDE relatif à la prévention, à la préparation et à l'intervention en matière d'accidents chimiques est effectué par le Groupe de travail sur les accidents chimiques, avec le soutien administratif de la Division environnement, santé et sécurité, relevant de la Direction de l'environnement $^{1}$ du Secrétariat de l'OCDE. Les objectifs du Programme sur les accidents chimiques comprennent l'échange de l'information et de l'expérience, l'analyse de questions spécifiques d'intérêt commun pour les pays membres de l'OCDE et l'élaboration de documents d'orientation. Dans cette perspective, plus de 15 ateliers et sessions spéciales ont été organisés depuis 1989. Pour obtenir soit de plus amples informations sur le Programme, soit la liste des documents d'orientation et autres publications préparés dans le cadre du Programme, veuillez consulter le site Internet suivant: www.oecd.org/env/accidents.

Les travaux du GTAC, en particulier l'élaboration des présents Principes directeurs, ont été menés en étroite collaboration avec d'autres organisations internationales. Un certain nombre d'entre elles, dont l'Organisation internationale du travail (OIT), le Programme des Nations Unies pour l'environnement, la Commission économique des Nations Unies pour l'Europe (CEE-ONU), l'Organisation mondiale de la santé (OMS) et le Bureau de la coordination des affaires humanitaires des Nations Unies (par l'entremise du Groupe commun PNUE/BCAH de l'environnement), sont très actives dans le domaine de la prévention, de la préparation et de l'intervention en matière d'accidents chimiques, et ont préparé des documents d'orientation sur des sujets connexes (pour connaître l'adresse Internet de ces organisations, veuillez consulter l'annexe IV). 


\section{Élaboration des présents Principes directeurs}

Il s'agit ici de la deuxième édition des Principes directeurs; elle constitue une version mise à jour et augmentée de la première édition, parue en 1992.

Afin de faciliter la préparation de cette deuxième édition, le GTAC a mis sur pied un groupe de rédaction formé de délégués des pays membres et des pays observateurs ainsi que de représentants de l'industrie, des travailleurs, d'organisations non gouvernementales et d'autres organisations internationales. On trouve la liste des membres de ce groupe dans la section réservée aux remerciements.

La principale source d'information utilisée pour préparer les Principes directeurs est une série de rapports issus d'ateliers et de sessions spéciales tenus entre 1989 et 2001 dans le but d'examiner la gamme, très vaste, des questions associées à la prévention, à la préparation et à l'intervention en cas d'accident, et d'étudier les rôles et responsabilités des diverses parties nécessairement engagées dans de telles activités, c'est-à-dire les pouvoirs publics à tous les niveaux, la direction des installations dangereuses ainsi que les autres employés de ces établissements et leurs représentants, et le public (notamment les collectivités vivant à proximité des installations dangereuses). Lors de tous ces ateliers, on a pu profiter du large éventail de connaissances spécialisées et d'avis de quelque 100 participants, parmi lesquels on comptait habituellement des représentants de toutes les parties concernées. Ces ateliers et sessions spéciales avaient notamment pour but de parvenir à une série de conclusions et de recommandations concernant les "meilleures pratiques", lesquelles ont été adaptées aux fins de l'élaboration des présents Principes directeurs².

Le Groupe de rédaction a également tenu compte, durant la préparation du présent document, des travaux de la Commission européenne et de nombreuses organisations internationales (OIT, OMI, BCAH, PNUE, CEE-ONU et OMS). Les documents produits par ces organisations fournissent de précieuses informations techniques permettant d'appuyer la mise en œuvre des principes généraux énoncés dans le présent document. Nombre de ces documents d'orientation sont répertoriés à l'annexe IV (sélection de références).

Afin de mieux vérifier encore la validité et la justesse des Principes directeurs, le GTAC en a fait largement circuler l'ébauche, de façon à obtenir les avis et suggestions de diverses parties, issues de pays membres de l'OCDE ou non, et des différentes organisations concernées. Une réunion extraordinaire du GTAC et d'autres parties intéressées a eu lieu en juin 2002 afin de revoir et d'intégrer, au besoin, les commentaires reçus. On trouve davantage de renseignements sur ceux qui ont participé à ce processus dans la section réservée aux remerciements. 


\section{NOTES}

1. La Division environnement, santé et sécurité œuvre dans divers domaines relatifs à la sécurité des substances chimiques, à la sécurité des biotechnologies et aux pesticides, et publie des documents dans six domaines à part les accidents chimiques, soit : essais et évaluation; principes de bonnes pratiques de laboratoire et vérification du respect de ces principes; scénarios d'émission; pesticides; gestion du risque; harmonisation de la surveillance réglementaire de la biotechnologie.

2. La liste complète des ateliers et des sessions spéciales se trouve à l'annexe IV. 


\section{ANNEXE VI}

\section{RÉSUMÉ DES « RÈGLES D'OR »}

\section{RÔLE DE TOUTES LES PARTIES PRENANTES}

" Pour protéger la santé, l'environnement et les biens, considérez prioritairement la réduction des risques et la prévention des accidents chimiques, ainsi que la préparation aux situations d'urgence et l'intervention en cas d'accident.

- Communiquez et coopérez avec les autres parties prenantes sur tous les aspects de la prévention, de la préparation et de l'intervention en matière d'accidents chimiques.

\section{RÔLE DE L'INDUSTRIE (direction et travailleurs)}

\section{Direction}

D Soyez au fait des dangers et des risques présents aux installations abritant des substances dangereuses.

" Faites prévaloir une « culture de la sécurité » connue et acceptée dans l'entreprise tout entière.

" Établissez des systèmes de gestion de la sécurité et surveillez/examinez leur application.

" Appliquez des principes technologiques à plus grande «sécurité intrinsèque » dans la conception et l'exploitation des installations dangereuses.

W Apportez un soin particulier à la gestion du changement.

w Préparez-vous à tout accident pouvant se produire.

* Aidez les autres à assumer leur rôle et leurs responsabilités.

1) Recherchez en permanence des possibilités d'amélioration.

\section{Travailleurs}

" Agissez conformément à la culture de la sécurité de l'entreprise, à ses procédures de sécurité et à la formation reçue.

N Ne ménagez aucun effort pour vous informer et communiquer à la direction les renseignements et toute information en retour.

v Prenez l'initiative de contribuer à informer et à sensibiliser votre communauté.

\section{RÔLE DES POUVOIRS PUBLICS}

" Élaborez des politiques, des règlements et des procédures, veillez à les faire appliquer et employezvous à les améliorer en permanence.

W Montrez la voie à suivre afin de motiver toutes les parties prenantes à remplir leur rôle et à assumer leurs responsabilités.

W Suivez de près l'industrie pour veiller à ce que les risques soient pris en compte de façon appropriée.

W Favorisez une communication et une coopération efficaces entre les parties prenantes.

w Favorisez la coordination entre les divers organismes

v Soyez au fait des risques relevant de votre domaine de compétence et planifiez en conséquence.

- Atténuez les effets des accidents par des mesures d'intervention appropriées.

D Prenez des mesures et des dispositions adaptées et cohérentes d'aménagement du territoire.

\section{RÔLE DES COLLECTIVITÉS ET DES PARTICULIERS}

W Soyez conscient des risques auxquels est exposée votre collectivité et sachez quoi faire en cas d'accident.

Participez à la prise de décisions concernant les installations dangereuses.

. Coopérez avec les autorités locales et l'industrie aux interventions d'urgence et à leur planification. 
PUBLICATION DE L'OCDE, 2, rue André-Pascal, 75775 PARIS CEDEX 16 IMPRIMÉ EN FRANCE

(97 2003101 P) ISBN 92-64-10181-0 - No 530212003 